\title{
INVESTIGATION OF EXPOSURE TO LIFESTYLE AND ENVIRONMENTAL FACTORS ON CUMULUS-OOCYTE COMPLEX FUNCTION
}

\section{KELLY ANNE CAMPEN}

A thesis submitted to Victoria University of Wellington in fulfilment of the requirements for the degree of Doctor of Philosophy in Biomedical Science

Victoria University of Wellington 



\begin{abstract}
Pathways involved in bi-directional communication within the cumulus-oocyte complex (COC) include gap junction (GJ) communication, oocyte growth factor production, and glucose metabolism and are essential for oocyte health. Perturbation of these pathways may result in reduced oocyte quality due to altered COC function. Using rats as a model, in vitro effects of exposure to bisphenol A (BPA), caffeine, nicotine, ethanol, methylenedioxymethamphetamine (MDMA), or $\Delta^{9}$-tetrahydrocannabinol (THC) on COC function were investigated. Furthermore, MDMA was administered to rats to compare in vitro with in vivo effects.
\end{abstract}

The transfer of a fluorescent dye (calcein) from cumulus cells (CC) to the oocyte was used as a measure of GJ communication. Expression of CC-derived (Atr, Cx43, Cycs, Gfpt1, Pfkp) and oocyte-derived (Atr, Bmp15, Cx37, Gdf9) genes were measured using multiplex TaqMan quantitative PCR. Levels of CX43 and GDF9 proteins were quantified using Western blotting.

Optimisation of the GJ bioassay included the addition of phosphodiesterase inhibitors (rolipram and dipyridamole), and a 1 hour post-calcein incubation period to allow dye transfer. Quantification of gene expression in calcein-treated CC and oocytes was validated, enabling direct comparisons between GJ communication and gene expression.

To determine the in vitro effects, $\mathrm{COC}$ were incubated with test factors at high physiological concentrations over 25 hours. GJ communication decreased over time in control COC. This reduction was attenuated after exposure to BPA and nicotine, and partially by caffeine. Furthermore, exposure to ethanol maintained oocyte meiotic arrest, whereas MDMA and THC promoted meiotic resumption.

Oocyte-derived gene expression was mostly unaffected by in vitro exposure to the lifestyle and environmental factors, although a treatment $x$ time interaction for $C \times 37$ levels following nicotine exposure was observed. Of the CC-derived genes, $C x 43$ was the most sensitive where BPA, MDMA, and THC increased, and caffeine and ethanol decreased, expression. In CC, exposure to MDMA and THC increased Gfpt1 levels and exposure to MDMA resulted in a treatment $\mathrm{x}$ time interaction in Cycs and Pfkp expression. 
In COC, caffeine increased CX43 protein levels after 1 hour. Nicotine initially reduced, but with time increased CX43 levels. Furthermore, CX43 levels decreased and increased after 25 hour exposures to ethanol and MDMA, respectively. GDF9 protein levels in COC exhibited wide within-treatment variation. Overall, BPA and caffeine reduced GDF9 levels after 1 hour whereas GDF9 levels were increased following exposure to BPA, caffeine, MDMA, and THC for 25 hours.

To determine in vivo effects, female rats were administered saline or $5 \mathrm{mg} / \mathrm{kg} /$ day MDMA for 3 days. COC from MDMA-treated rats had higher levels of CX43 protein but gene expression and meiotic reactivation were unaffected.

In conclusion, COC function was altered by in vitro exposure to BPA, caffeine, ethanol, nicotine, MDMA, and THC. Furthermore, in vivo exposure to MDMA elicits similar, albeit reduced, effects on $\mathrm{COC}$ function. A role for $\mathrm{CC}$ in protecting the oocyte from harmful contaminants is proposed. Perturbation of the bi-directional communication pathway is likely to influence oocyte quality due to alterations in nutrient availability and timing of follicular events, although these may not be associated with negative outcomes. This study provides evidence that exposure to lifestyle factors and environmental contaminants affect COC function. 


\section{ACKNOWLEDGEMENTS}

First of all I would like to thank my supervisors, Janet Pitman and Ken McNatty. Thank you for always being available to discuss ideas and results, while still giving me the freedom to make this project my own. I have had such a good experience doing this research project, and that would not have been possible without the supportive, friendly environment that Ken and Janet have set up in the Reproductive Biology group at Victoria.

Thank you also to the other members of the Reproduction group, both past and present. I am especially grateful to Norma Hudson, who helped me with dealing with the rats, and with the gap junction assay and tissue culture aspects of the project. My thanks also go to Adrian Bibby, Joy Mclntosh, and Derek Heath for their assistance with the Western blotting. Finally, thanks to all the students from the Reproduction group for all the coffee and cake breaks and for their friendship.

This project would not have been possible without the generosity of Sue Schenk, Richard Moore, and Peter Vancompernolle who provided the female rats used for my experiments. In addition, Richard and Sue donated the MDMA and provided invaluable advice on the in vivo experiment and the preparation of my Animal Ethics application. Thank you to Sarah Bradbury and Dane Aronsen who performed the ip injections for the in vivo study and who taught me to use the locomotor activity boxes. I am also grateful to Ryan Steel and Darren Day who donated the THC resin used in this study.

I would never have gotten to this point without my wonderful family. Thank you to Mum, Hannah, Nana and Poppa for all your love and support over the years. My thanks also go to the Campen, Bright and Neveu families, especially lan for taking us under his roof these last few months. Finally, my eternal love and thanks go to my husband Richard. You have contributed to my success in too many ways to name. Thank you for your pride in me, for your undying encouragement, and for making life so much brighter. 


\section{TABLE OF CONTENTS}

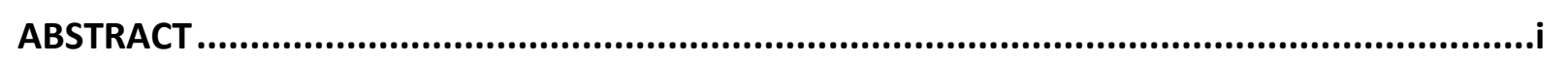

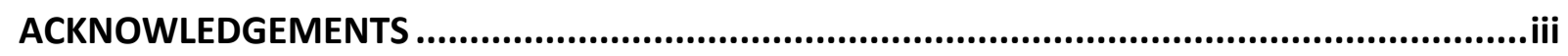

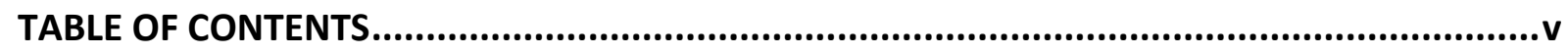

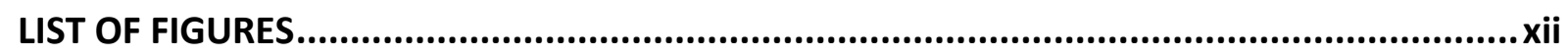

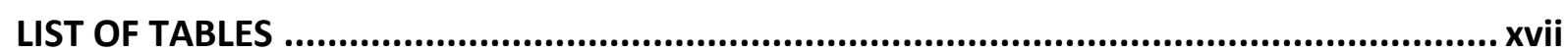

LIST OF COMMONLY USED ABBREVIATIONS .......................................................... xviii

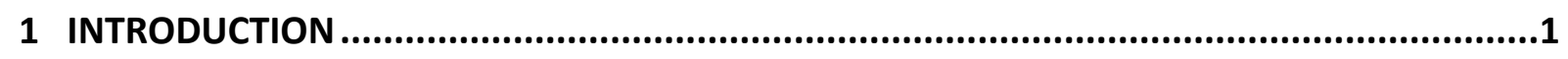

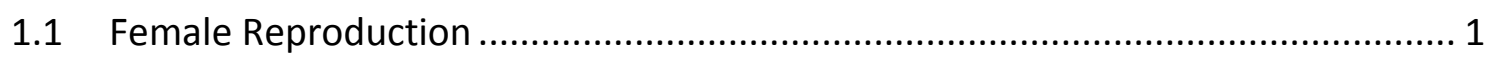

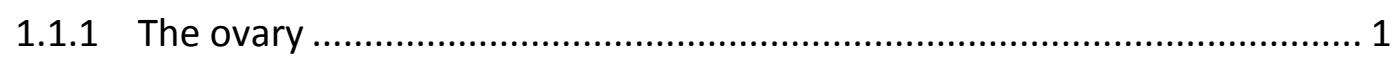

1.1.2 Ovarian follicular formation and growth ......................................... 1

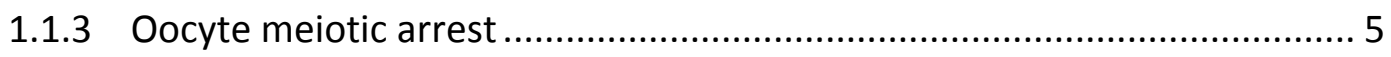

1.1.4 The pre-ovulatory LH surge and ovulation ........................................ 7

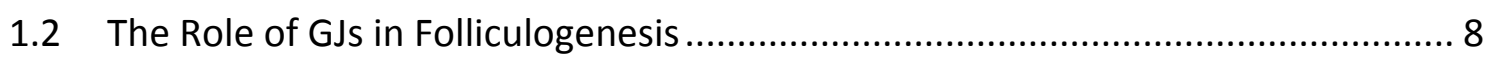

1.2.1 General GJ structure and function .................................................. 8

1.2.2 The Role of GJs in Folliculogenesis .................................................. 12

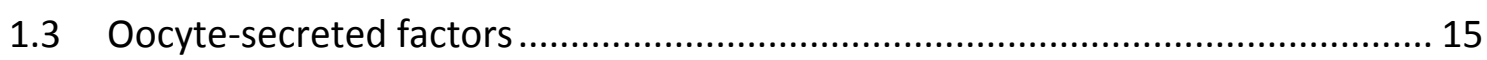

1.3.1 Oocyte-secreted factors and follicular development........................... 15

1.3.2 Oocyte-secreted factors and follicular metabolic functions .................... 19

1.3.3 GDF9 and BMP15 expression and signalling in the ovary ...................... 20

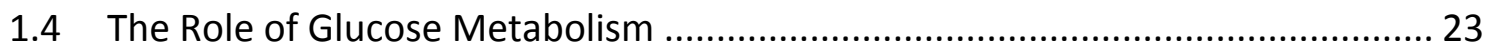

1.4.1 Overview of glucose metabolic pathways ........................................... 23

1.4.2 Glucose metabolism in the ovarian follicle ........................................ 27

1.5 The Influence of Exogenous Factors on Oocyte Quality ................................... 30

1.5.1 Lifestyle factors chosen for this study ............................................. 33

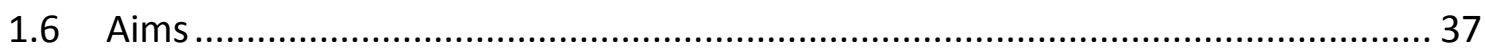




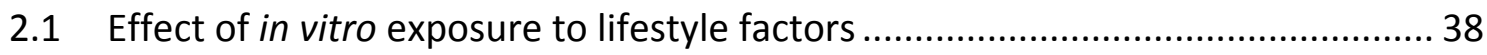

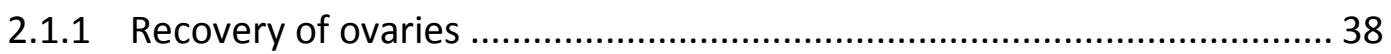

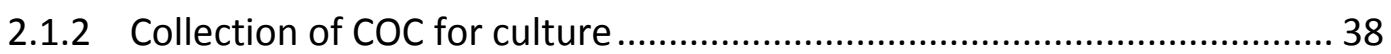

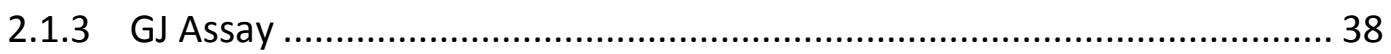

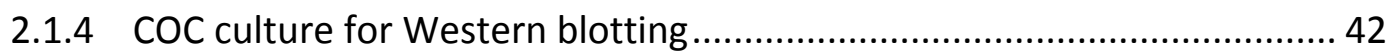

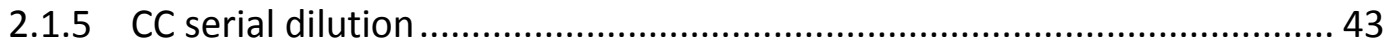

2.2 Effect of in vivo exposure to methylenedioxymethamphetamine (MDMA) ........ 43

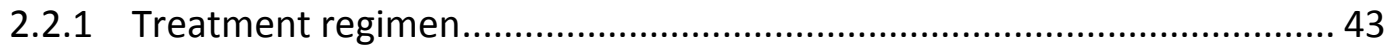

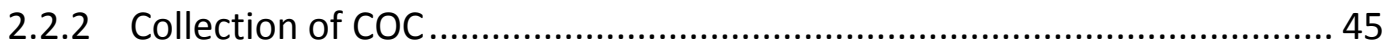

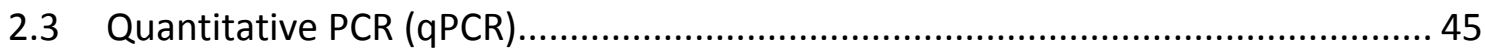

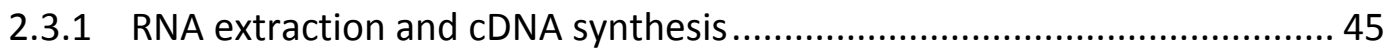

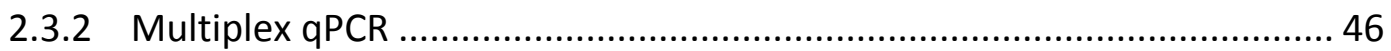

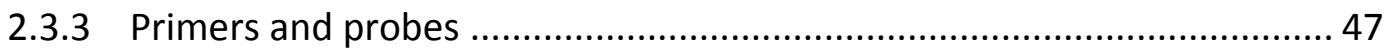

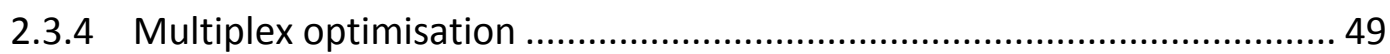

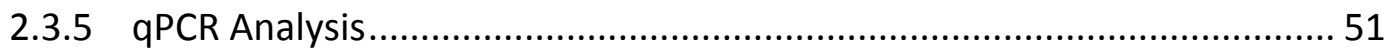

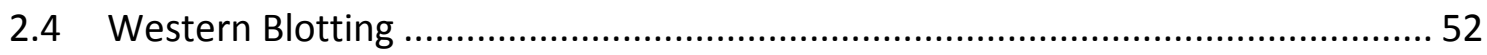

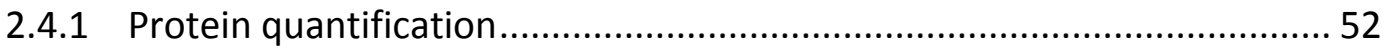

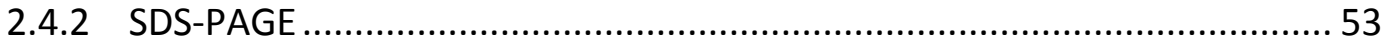

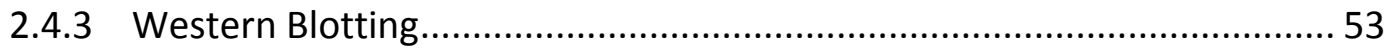

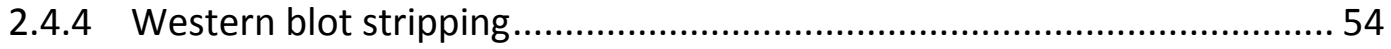

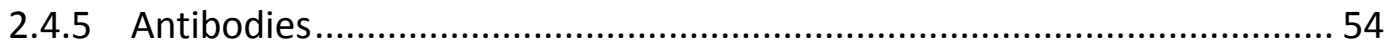

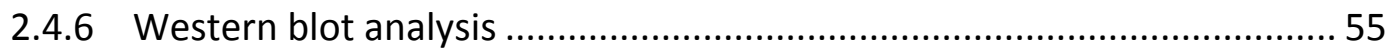

3 VALIDATION OF A GJ BIOASSAY TO DETERMINE GJ ACTIVITY IN COC...........................56

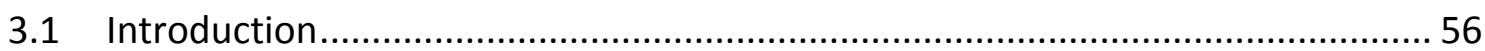

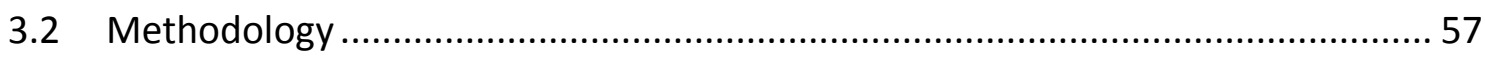




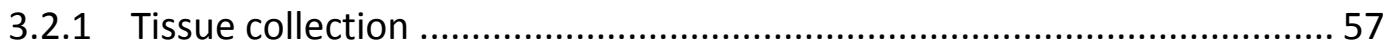

3.2.2 Dose-response effects of DMSO concentrations ................................. 57

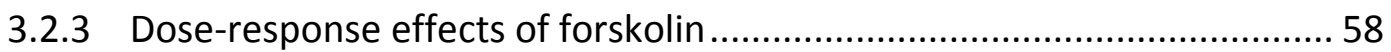

3.2.4 Dose-response effects of single PDE inhibitors .................................... 58

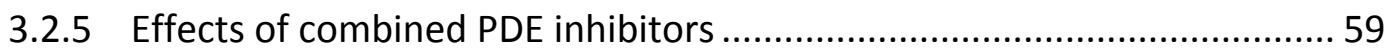

3.2.6 Effects of time on dye transfer to the oocyte .......................................... 59

3.2.7 Testing the specificity of the GJ bioassay ............................................ 59

3.2.8 Testing the robustness of the GJ bioassay .......................................... 60

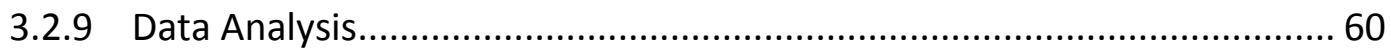

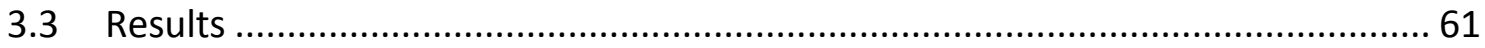

3.3.1 Dose-response effects of DMSO concentrations ....................................6 61

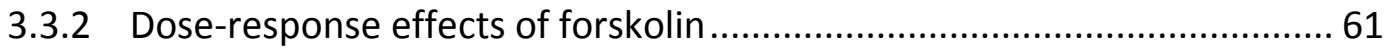

3.3.3 Dose-response effects of single PDE inhibitors .......................................63 63

3.3.4 Effects of combined PDE inhibitors ....................................................... 67

3.3.5 Effects of time on dye transfer to the oocyte ....................................... 68

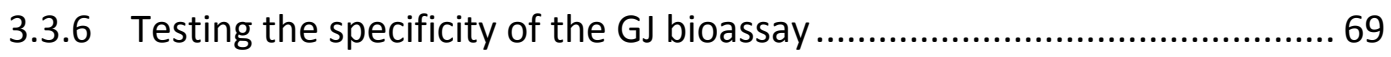

3.3.7 Testing the robustness of the GJ bioassay ......................................... 70

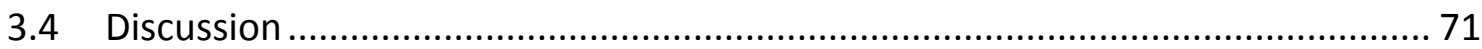

4 VALIDATING THE USE OF COC PREVIOUSLY UTILISED IN GJ ASSAYS FOR GENE

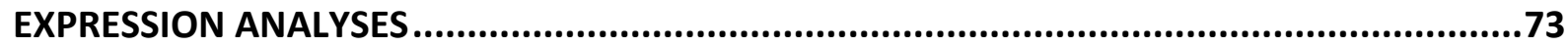

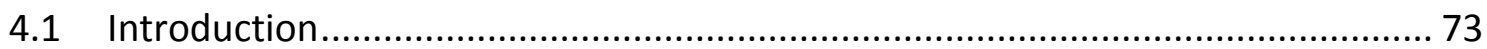

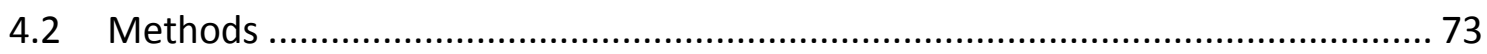

4.2.1 Detection of calcein fluorescence in qPCR reactions ............................ 74

4.2.2 Correlation of Rpl19 $\mathrm{C}_{\mathrm{T}}$ value to $\mathrm{CC}$ number and oocyte diameter........... 74

4.2.3 Effect of calcein/calcein-AM on expression of candidate genes.............. 75

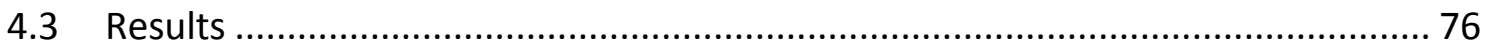


4.3.1 Detection of calcein fluorescence ..................................................... 76

4.3.2 Correlation of Rpl19 $\mathrm{C}_{\mathrm{T}}$ value to $\mathrm{CC}$ number and oocyte diameter.......... 78

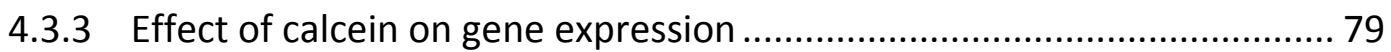

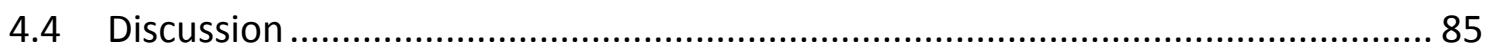

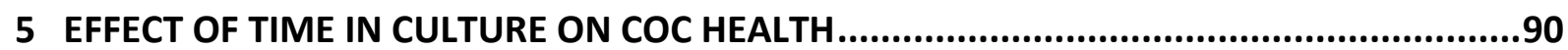

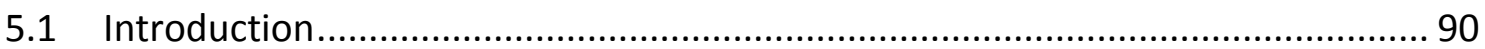

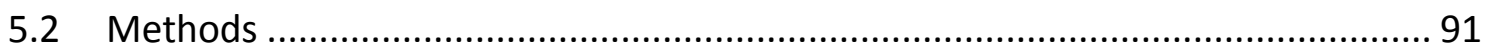

5.2.1 Effect of time on GJ communication ................................................. 91

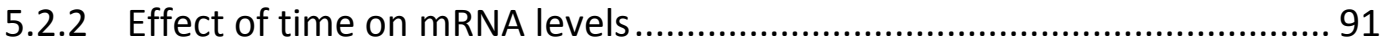

5.2.3 Effect of time on protein expression................................................. 92

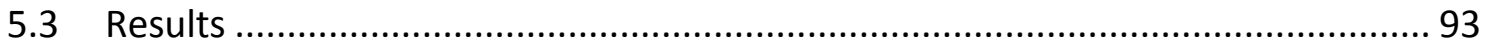

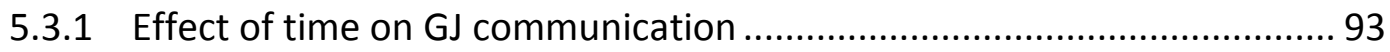

5.3.2 Effect of time on mRNA levels in rat oocytes and CC............................. 94

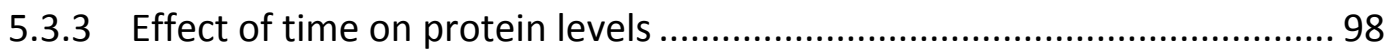

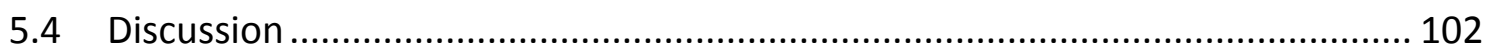

6 EFFECTS OF IN VITRO EXPOSURE OF COC TO LIFESTYLE FACTORS ON CC-OOCYTE GJ COMMUNICATION .......................................................................................................109

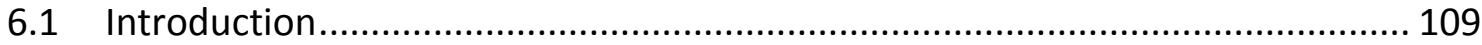

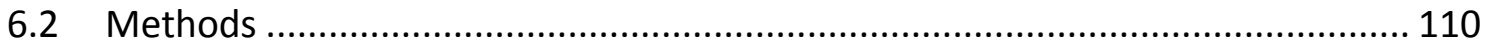

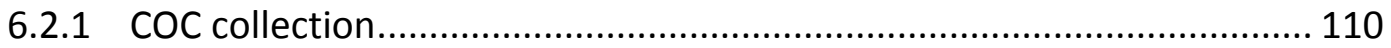

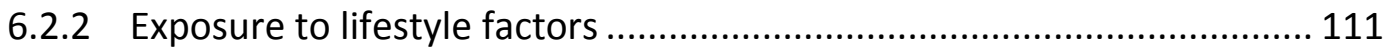

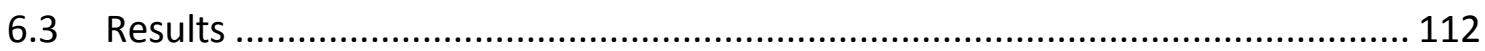

6.3.1 Effect of BPA on GJ communication in rat COC .................................. 112

6.3.2 Effect of caffeine on GJ communication in rat COC ............................ 113

6.3.3 Effect of ethanol on GJ communication in rat COC ............................. 115

6.3.4 Effect of nicotine on GJ communication in rat COC ........................... 116 
6.3.5 Effect of MDMA on GJ communication in rat COC.

6.4 Discussion

7 EFFECTS OF IN VITRO EXPOSURE OF COC TO LIFESTYLE FACTORS ON GENE EXPRESSION IN OOCYTES 126

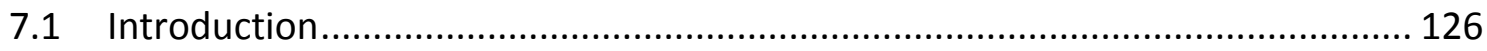

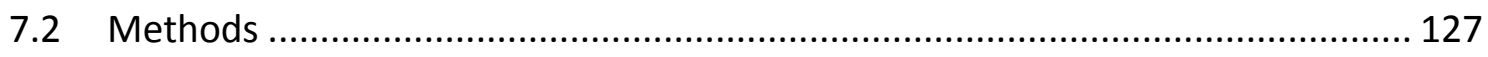

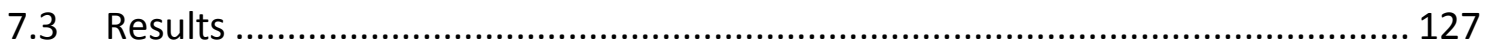

7.3.1 Effect of BPA on gene expression in rat oocytes................................ 127

7.3.2 Effect of caffeine on gene expression in rat oocytes ......................... 128

7.3.3 Effect of ethanol on gene expression in rat oocytes ............................. 130

7.3.4 Effect of nicotine on gene expression in rat oocytes ............................ 130

7.3.5 Effect of MDMA on gene expression in rat oocytes............................. 130

7.3.6 Effect of THC on gene expression in rat oocytes.............................. 130

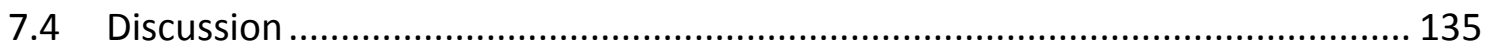

\section{EFFECTS OF IN VITRO EXPOSURE OF COC TO LIFESTYLE FACTORS ON GENE EXPRESSION}

IN CC 137

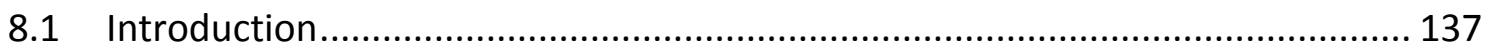

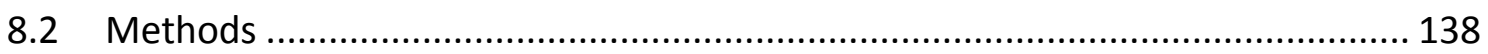

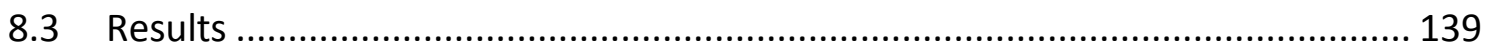

8.3.1 Effect of BPA on gene expression in rat CC ......................................... 139

8.3.2 Effect of caffeine on gene expression in rat CC................................. 139

8.3.3 Effect of ethanol on gene expression in rat CC ................................ 142

8.3.4 Effect of nicotine on gene expression in rat CC ............................... 142

8.3.5 Effect of MDMA on gene expression in rat CC .................................... 145

8.3.6 Effect of THC on gene expression in rat CC ...................................... 145 
8.4 Discussion

9 EFFECTS OF IN VITRO EXPOSURE OF COC TO LIFESTYLE FACTORS ON PROTEIN

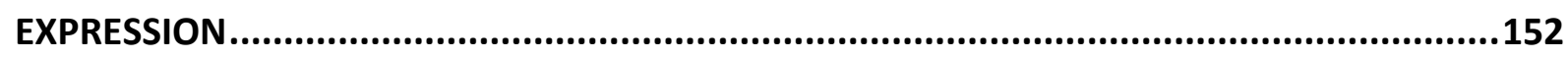

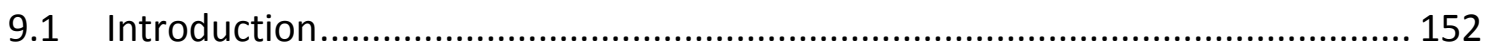

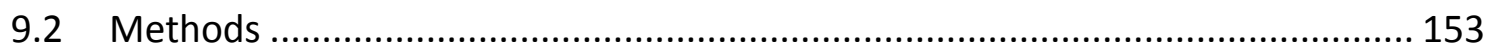

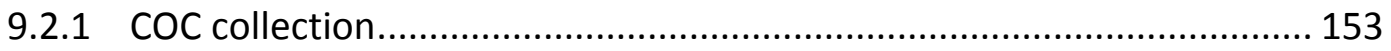

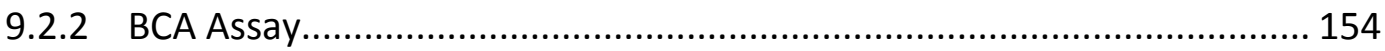

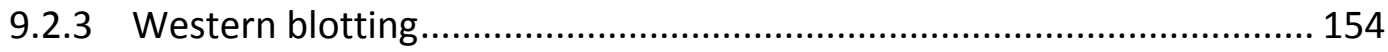

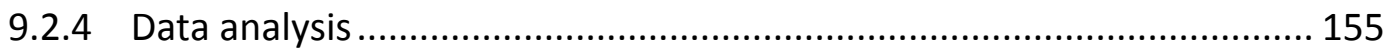

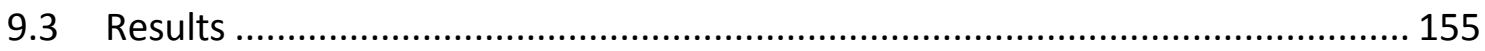

9.3.1 Effect of BPA and caffeine on protein levels in rat COC........................ 155

9.3.2 Effect of ethanol on protein levels in rat COC .................................... 161

9.3.3 Effect of nicotine on protein levels in rat COC .................................. 166

9.3.4 Effect of MDMA and THC on protein levels in rat COC ...................... 172

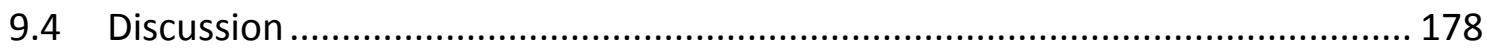

10 EFFECTS OF IN VIVO EXPOSURE TO MDMA ON COC FUNCTION ..................................185

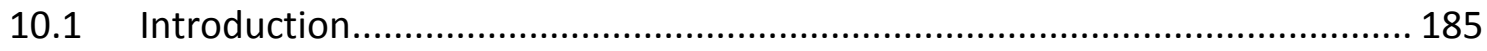

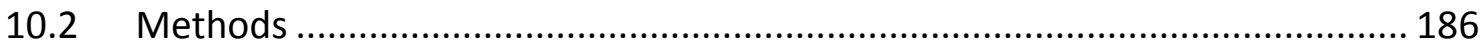

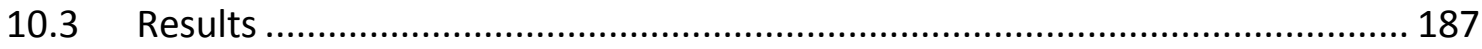

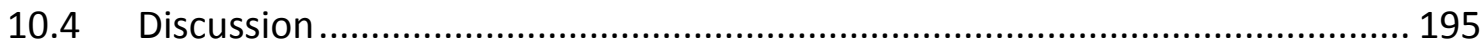

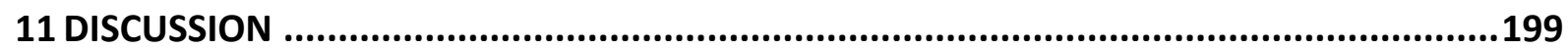

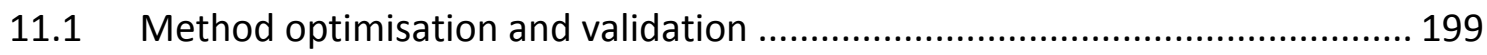

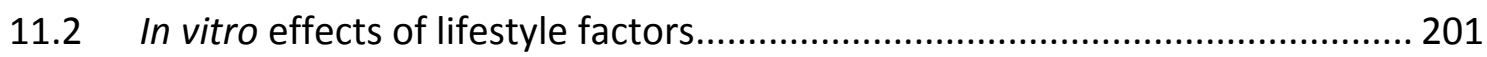

11.2.1 GJ communication is promoted by BPA, caffeine, and nicotine .......... 204

11.2.2 GDF9 protein levels are altered by BPA, caffeine, MDMA and THC ..... 207

11.2.3 GVBD is prevented by ethanol but promoted by MDMA and THC...... 208 
11.2.4 MDMA and THC alter expression of genes involved in glucose

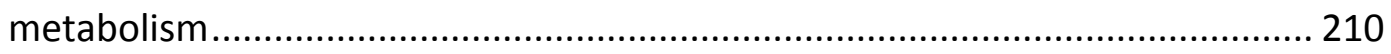

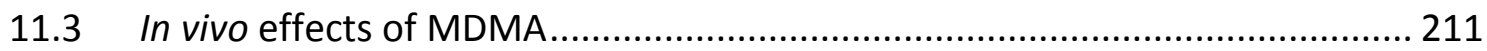

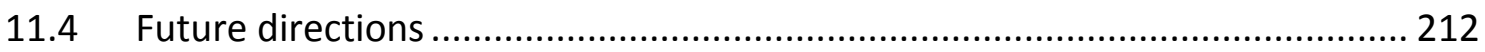

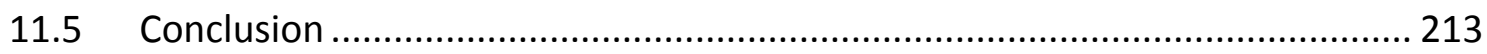

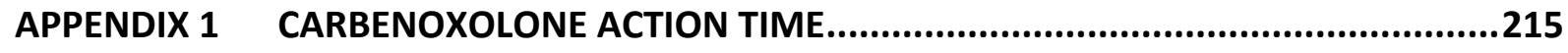

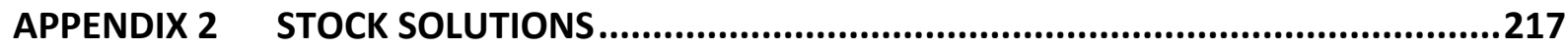

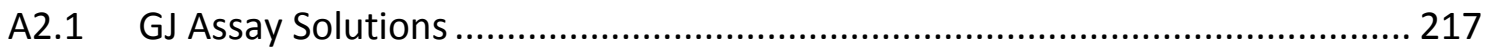

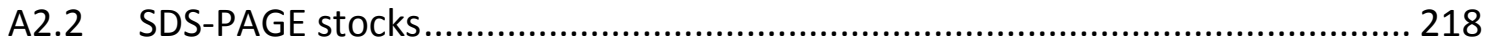

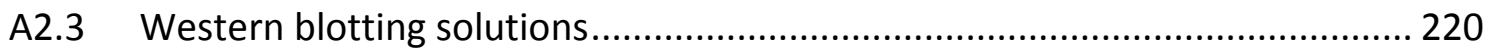

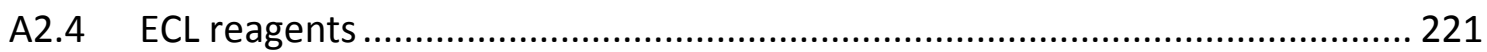

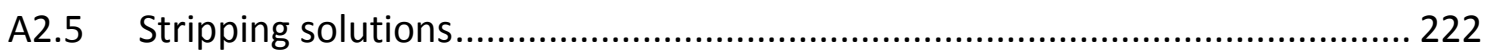

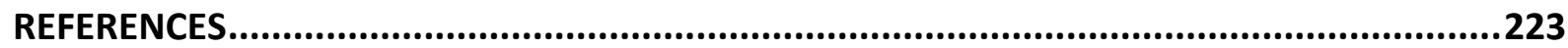




\section{LIST OF FIGURES}

Figure 1-1: The structure of the ovarian follicle during different growth stages..................... 3

Figure 1-2: Sheep ovarian follicles at various stages of development.................................... 4

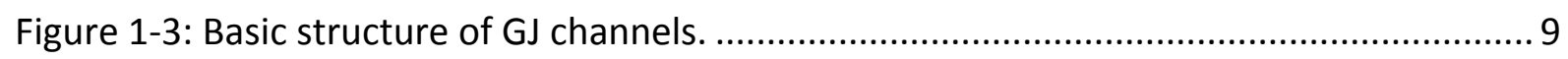

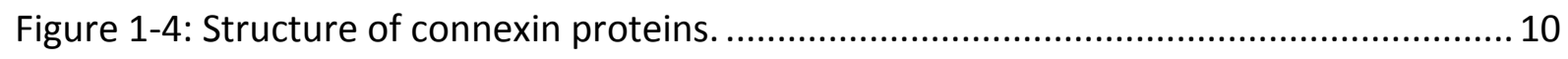

Figure 1-5: Schematic of a GJ bioassay utilising calcein to measure GJ communication

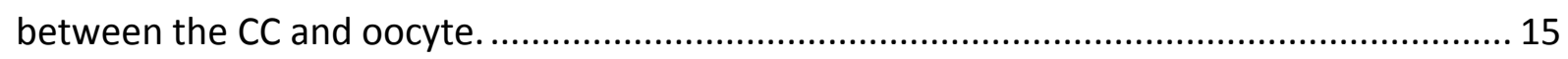

Figure 1-6: Schematic overview of the TGF $\beta$ family signalling pathway. .............................. 22

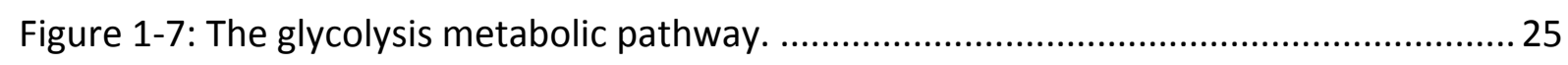

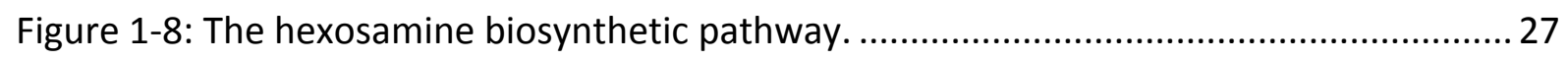

Figure 1-9: Potential sites of action for disruption of female fertility by exogenous factors.. 32 Figure 2-1: Photographs depicting rat $\operatorname{COC}(A, C)$ and denuded oocytes $(B, D)$ before and after exposure to calcein-AM. Indicated on the photographs are the cumulus cells (CC), oocyte (O), germinal vesicle (GV), and zona pellucida (ZP).

Figure 2-2: Schematic overview of the protocol used to optimise dye transfer in the GJ assay.

Figure 2-3: Photographs depicting an oocyte with a visible germinal vesicle $(A)$ and without a germinal vesicle (B).

Figure 2-4: Schematic overview of the optimised GJ assay protocol.

Figure 3-1: Effect of differing concentrations of DMSO on fluorescence transfer from adjacent CC to oocytes after a 4 hour incubation of rat COC.

Figure 3-2: Effect of forskolin on fluorescent dye transfer from adjacent CC to oocytes after a 4 hour incubation of rat COC.

Figure 3-3: Effect of differing concentrations of milrinone on fluorescent dye transfer from adjacent CC to oocytes after a 4 hour incubation of rat COC.

Figure 3-4: Effect of differing concentrations of dipyridamole on fluorescent dye transfer from adjacent CC to oocytes after a 4 hour incubation of rat COC. .65

Figure 3-5: Effect of differing concentrations of rolipram on fluorescent dye transfer from adjacent CC to oocytes after a 4 hour incubation of rat COC. .66

Figure 3-6: Effect of single or combinations of PDE inhibitors on fluorescent dye transfer from adjacent $\mathrm{CC}$ to oocytes after a 4 hour incubation of rat $\mathrm{COC}$.

Figure 3-7: Effect of time on fluorescent dye transfer from adjacent CC to oocytes. 69 
Figure 3-8: Effect of increasing concentrations of carbenoxolone on fluorescent dye transfer from adjacent CC to oocytes after a 4 hour incubation of rat COC.

Figure 3-9: Effect of $1 \mathrm{mM}$ carbenoxolone on fluorescent dye transfer from adjacent CC to oocytes after 5,13 , and 25 hours incubation in rat COC.

Figure 4-1: Detection of fluorescence in a $40 \mu \mathrm{M}$ calcein-AM solution over 40 cycles. .76

Figure 4-2: Detection of fluorescence in DO that had been incubated with and without calcein over 40 cycles. .77

Figure 4-3: Detection of fluorescence in cDNA samples of oocytes that had been incubated with and without calcein over 40 cycles. .77

Figure 4-4: Correlation between $R p / 19 C_{T}$ value and CC number $(A)$ or oocyte diameter (B). 78 Figure 4-5: Effect of calcein-AM with time on Rp/19 expression levels in oocytes (A) and CC (B). .80

Figure 4-6: Effect of calcein-AM with time on mRNA levels of $\operatorname{Atr}(A), B m p 15$ (B), Cx37 (C), Gdf9 (D) in rat oocytes.

Figure 4-7: Effect of calcein-AM with time on mRNA levels of Atr (A), Cx43 (B), Cycs (C), Gfpt1 (D), and $P f k p(E)$ in rat CC. 84

Figure 5-1: Effect of incubation time on fluorescent dye transfer from adjacent CC to oocytes. 93

Figure 5-2: Effect of incubation time on mRNA levels of $\operatorname{Atr}(\mathrm{A}), B m p 15$ (B), Cx37 (C), Gdf9 (D), and $R p / 19$ (E) in rat oocytes. 95

Figure 5-3: Effect of incubation time on mRNA levels of Atr (A), Cx43 (B), Cycs (C), Gfpt1 (D), $P f k p(\mathrm{E})$, and $R p / 19(\mathrm{~F})$ in rat CC masses. 96

Figure 5-4: Correlation between oocyte fluorescence values and $C_{T}$ values for $C_{X} 43(A)$ and Cx37 (B). .97

Figure 5-5: The in vitro effect of incubation time on CX43 protein levels in rat COC. 99

Figure 5-6: Effect of incubation time on relative CX43 levels in rat COC. 100

Figure 5-7: The in vitro effects of incubation time on GDF9 protein levels in rat COC. 101 Figure 5-8: Effect of incubation time on promature (A) and mature (B) GDF9 protein levels in rat COC. 102

Figure 6-1: Effect of BPA on fluorescent dye transfer from adjacent CC to oocytes over time in vitro. 
Figure 6-2: Effect of caffeine on fluorescent dye transfer from adjacent CC to oocytes over time.

Figure 6-3: Effect of ethanol on fluorescent dye transfer from adjacent CC to oocytes over time in vitro.

Figure 6-4: Effect of nicotine on fluorescent dye transfer from adjacent $\mathrm{CC}$ to oocytes over time in vitro.

Figure 6-5: Effect of MDMA on fluorescent dye transfer from adjacent CC to oocytes over time.

Figure 6-6: Effect of THC on fluorescent dye transfer from adjacent CC to oocytes over time in vitro

Figure 7-1: Effect of BPA on mRNA levels of $\operatorname{Atr}(\mathrm{A}), B m p 15$ (B), Cx37 (C), Gdf9 (D), and Rp/19 (E) in rat oocytes. 128

Figure 7-2: Effect of caffeine on mRNA levels of Atr (A), Bmp15 (B), Cx37 (C), Gdf9 (D), and Rp/19 (E) in rat oocytes. 129

Figure 7-3: Effect of ethanol on mRNA levels of $\operatorname{Atr}(A), B m p 15$ (B), CX37 (C), Gdf9 (D), and Rp/19 (E) in rat oocytes.

Figure 7-4: Effect of nicotine on mRNA levels of $\operatorname{Atr}(A), B m p 15$ (B), Cx37 (C), Gdf9 (D), and $R p / 19(E)$ in rat oocytes.

Figure 7-5: Effect of MDMA on mRNA levels of Atr (A), Bmp15 (B), Cx37 (C), Gdf9 (D), and Rp/19 (E) in rat oocytes.

Figure 7-6: Effect of THC on mRNA levels of Atr (A), Bmp15 (B), Cx37 (C), Gdf9 (D), and Rp/19 (E) in rat oocytes. 134

Figure 8-1: Effect of BPA on mRNA levels of Atr (A), Cx43 (B), Cycs (C), Gfpt1 (D), Pfkp (E), and $R p / 19(\mathrm{~F})$ in rat CC. 140

Figure 8-2: Effect of caffeine on mRNA levels of $\operatorname{Atr}(A), C x 43$ (B), Cycs (C), Gfpt1 (D), Pfkp (E), and $R p / 19$ (F) in rat CC.

Figure 8-3: Effect of ethanol on mRNA levels of $\operatorname{Atr}(A), C x 43$ (B), Cycs (C), Gfpt1 (D), Pfkp (E), and $R p / 19$ (F) in rat CC.

Figure 8-4: Effect of nicotine on mRNA levels of $\operatorname{Atr}(A), C x 43$ (B), Cycs (C), Gfpt1 (D), Pfkp (E), and $R p / 19$ (F) in rat CC. 144

Figure 8-5: Effect of MDMA on mRNA levels of $\operatorname{Atr}(A), C x 43$ (B), Cycs (C), Gfpt1 (D), Pfkp (E), and $R p / 19$ (F) in rat CC. 
Figure 8-6: Effect of THC on mRNA levels of Atr (A), Cx43 (B), Cycs (C), Gfpt1 (D), Pfkp (E), and Rp/19 (F) in rat CC.

Figure 9-1: The effects of exposing rat COC to BPA and caffeine in vitro on CX43 protein levels over time.

Figure 9-2: Effect of BPA and caffeine on relative CX43 levels in rat COC. 158

Figure 9-3: The effects of exposing rat COC to BPA and caffeine in vitro on GDF9 protein levels over time. 160

Figure 9-4: Effect of BPA and caffeine on relative GDF9 levels in rat COC. 161

Figure 9-5: The effects of exposing rat $\mathrm{COC}$ to ethanol in vitro on CX43 protein levels over time.

Figure 9-6: Effect of ethanol on relative CX43 levels in rat COC. 163

Figure 9-7: The effects of exposing rat COC to ethanol in vitro on GDF9 protein levels over time. 165

Figure 9-8: Effect of ethanol on relative GDF9 levels in rat COC. 166

Figure 9-9: The effects of exposing rat COC to nicotine in vitro on CX43 protein levels in rat COC over time. 168

Figure 9-10: Effect of nicotine on relative CX43 levels in rat COC. 169

Figure 9-11: The effects of exposing rat COC to nicotine in vitro on GDF9 protein levels over time.

Figure 9-12: Effect of nicotine on relative GDF9 levels in rat COC.

Figure 9-13: The effects of exposing rat COC to MDMA and THC in vitro on CX43 protein levels over time. 174

Figure 9-14: Effect of MDMA and THC on relative CX43 levels in rat COC. 175

Figure 9-15: The effects of exposing rat COC to MDMA and THC in vitro on GDF9 protein levels over time. 177

Figure 9-16: Effect of MDMA and THC on relative GDF9 levels in rat COC. .......................178

Figure 10-1: Effects of MDMA on ambulatory counts in female rats. 188

Figure 10-2: The effect of MDMA on candidate gene expression in CC (A) and oocytes (B) in female rats 190

Figure 10-3: The effect of MDMA on CX43 protein levels in COC from female rats. 192

Figure 10-4: Effect of in vivo exposure to MDMA on relative CX43 levels in rat COC. 193

Figure 10-5: Effect of MDMA on GDF9 protein levels in COC from female rats. 194 
Figure 10-6: Effect of in vivo exposure to MDMA on relative promature GDF9 levels in rat COC. 195

Figure A1-1: Effect of exposure time to $1 \mathrm{mM}$ carbenoxolone on fluorescent dye transfer from adjacent CC to oocytes after a 4 hour incubation of rat COC. 216 


\section{LIST OF TABLES}

Table 1-1: Measured concentrations of lifestyle factors in biological fluids.

Table 2-1: Nucleotide sequences for forward and reverse primers for qPCR, including NCBI accession numbers, for each gene investigated.

Table 2-2: Nucleotide sequences for TaqMan probe sequences for qPCR for each gene investigated.

Table 2-3: Optimal final concentrations ( $\mathrm{nM}$ ) of forward and reverse primers and TaqMan probes in qPCR reactions.

Table 2-4: The amplification efficiency for each gene in three different qPCR quadriplex reaction sets.

Table 3-1: Concentrations of PDE inhibitors used for the GJ dose-response experiments..... 58 Table 3-2: Effect of differing concentrations of milrinone on the proportion of oocytes in which a GV was observed.

Table 3-5: Effect of single or combinations of PDE inhibitors on the proportion of oocytes in which a GV was observed.

Table 4-1: Excitation and emission wavelengths for the green, orange, red, and yellow channels of the Corbett Rotor Gene 6000 ...... .74

Table 4-3: Summary of calcein-AM treatment, time, and interaction effects on mRNA levels of candidate genes in rat CC. .85

Table 6-1: Stock and final concentrations of treatment reagents in media.

Table 6-3: Effect of caffeine on the proportion of oocytes with a visible GV. 115

Table 6-6: Effect of MDMA on the proportion of oocytes with a visible GV.

Table 10-1: Effect of MDMA on the proportion of oocytes with a visible GV in female rats.189

Table 11-1: Summary of the in vitro effects of lifestyle factors on COC function. 202

Table A1-1: Incubation conditions for determining time required for maximal inhibition of dye transfer by $1 \mathrm{mM}$ carbenoxolone. 215 


\section{LIST OF COMMONLY USED ABBREVIATIONS}

AM

ANOVA

ATP

ATR

BCA

BMP15

BPA

CAMP

CC

CDNA

CEEF

cGMP

$\mathrm{COC}$

$\mathrm{C}_{\mathrm{T}}$

CX

CYCS

CYP

$\mathrm{dH}_{2} \mathrm{O}$

DMSO

DO

$\mathrm{ECL}$

$\mathrm{FSH}$

GC

GDF9

GFPT1

GJ

GLUT

GnRH

GV

GVBD
Acetoxymethyl

Analysis of variance

Adenosine triphosphate

Ataxia telangiectasia and Rad3 related

Bicinchoninic assay

Bone morphogenetic protein 15

Bisphenol A

Cyclic adenosine monophosphate

Cumulus cell

Complimentary deoxyribonucleic acid

Cumulus cell expansion enabling factor

Cyclic guanosine monophosphate

Cumulus-oocyte complex

Cycle threshold

Connexin

Cytochrome c, somatic

Cytochrome P450

Distilled water

Dimethyl sulfoxide

Denuded oocyte

Electrochemiluminescence

Follicle-stimulating hormone

Granulosa cell

Growth differentiation factor 9

Glutamine-fructose-6-phosphate transaminase 1

Gap junction

Glucose transport

Gonadotrophin releasing hormone

Germinal vesicle

Germinal vesicle breakdown 


\begin{tabular}{|c|c|}
\hline hCG & Human chorionic gonadotrophin \\
\hline HHMA & 3,4-dihydroxymethamphetamine \\
\hline ip & Intraperitoneal \\
\hline IVF & In vitro fertilisation \\
\hline IVM & In vitro maturation \\
\hline KO & Knockout \\
\hline $\mathrm{LH}$ & Luteinising hormone \\
\hline MAPK & Mitogen activated protein kinase \\
\hline MDA & 3,4-methylenedioxyamphetamine \\
\hline MDMA & Methylenedioxymethamphetamine \\
\hline MII & Meiosis II \\
\hline mRNA & Messenger ribonucleic acid \\
\hline oox & Oocytectomised \\
\hline PBS & Phosphate buffered saline \\
\hline PDE & Phosphodiesterase \\
\hline PFKP & Phosphofructokinase, platelet \\
\hline PKA & Protein kinase $A$ \\
\hline PPP & Pentose phosphate pathway \\
\hline qPCR & Quantitative polymerase chain reaction \\
\hline RPL19 & $60 S$ ribosomal protein L19 \\
\hline SDS-PAGE & Sodium dodecyl sulphate polyacrylamide gel electrophoresis \\
\hline SEM & Standard error of the mean \\
\hline SMAD & Similar to mothers against decapentaplegic \\
\hline TBS & Tris buffered saline \\
\hline TCA & Tricarboxylic acid \\
\hline TGF $\beta$ & Transforming growth factor $\beta$ \\
\hline THC & $\Delta^{9}$-tetrahydrocannabinol \\
\hline XOD & Xanthine oxidoreductase \\
\hline
\end{tabular}




\section{INTRODUCTION}

\subsection{Female Reproduction}

\subsubsection{The ovary}

Female mammals have two ovaries located in the abdominal cavity on either side of the uterus. The ovary consists of a medulla and cortex enclosed by an epithelial layer called the ovarian surface epithelium. The cortical region contains the ovarian follicles, which are composed of an egg (oocyte) surrounded by a layer or layers of somatic cells and a basal lamina. The medulla region contains the main blood and lymphatic vessels.

\subsubsection{Ovarian follicular formation and growth}

Germ cells are first identified in the yolk sac during early embryonic life in mammals. They migrate from here to the gonadal ridge and differentiate into oogonia. These proliferating oogonia continue to migrate towards the ovarian cortex and, together with adjacent mesynchymal cells (pregranulosa cells), eventually coalesce into a convoluted array of ovigerous cords. Enclosed within these cords, the oogonia continue to proliferate but now are limited to recruiting pregranulosa cells from the proliferating ovarian surface epithelium. During this process, the oogonia enter meiosis and arrest at the diplotene stage of prophase I (see Sawyer et al., 2002). As the oogonia enter meiosis, many undergo apoptosis and their associated pregranulosa cells dissociate from these degenerating oogonia and re-attach to surviving oocytes. Prior to birth, individual meiotically-arrested oocytes surrounded by a single layer of squamous pregranulosa cells and enclosed by a basement membrane emerge from the base of the ovigerous cords as primordial follicles (Aerts and Bols, 2010). The assembly of primordial follicles is completed prior to birth in primates and domestic species, and during neonatal life in rodents (Rajah et al., 1992; Van den Hurk et al., 2000; Sawyer et al., 2002). It is generally accepted that this pool of non-growing primordial follicles is nonrenewable although this view has been contested (Johnson et al., 2004).

Follicles initiate growth one at a time from the moment they are formed. Once growth has been initiated, the follicle grows without rest until it either becomes atretic and dies, or undergoes ovulation. Initiation of follicular growth takes place continuously regardless of the stage of reproductive life or time of the oestrous or menstrual cycle. This continuum is only 
terminated at death or when the pool of follicles is depleted such as at menopause in humans. The mechanism by which individual primordial follicles are recruited into the growing pool is largely unknown. Activation of growth of primordial follicles is likely to occur as a result of both stimulating factors and removal of inhibitory factors. Several pathways have been identified to be involved in primordial follicle activation including KIT ligand signalling, NOBOX and SOHLH1 transcription factor activation, and anti-Mullerian hormone (Edson et al., 2009). The transition from the primordial to primary follicle stage is characterised by a change in granulosa cell (GC) shape, from flattened to cuboidal (Figure 1-1). All growing follicles that reach the developmental stage that requires support from pituitary hormones will undergo atresia if these hormone concentrations are inappropriate such as any time before puberty (McNatty, 2000), during anoestrus or during certain stages of the oestrous cycle (Hirshfield, 1991).

Progression from the primary stage to the secondary stage involves enlargement of the oocyte and proliferation of the GC to form multiple concentric layers. Theca cells, derived from the interstitial stroma, surround the basal lamina sometime after the primary stage of growth (Edson et al., 2009). Two distinct layers, the theca interna and externa, become evident by the late preantral (i.e. secondary) stages of development: the theca externa is a less-well differentiated layer while the more differentiated and vascularised theca interna region is localised adjacent to the follicular basal lamina. The theca interna is the major source of follicular androgens, notably androstenedione. Androstenedione is a primary substrate for follicular oestradiol production. During antral follicular growth, androstenedione is metabolised to oestradiol by the P450 aromatase enzyme present in the GC (Magoffin, 2005). After initiation of growth, a glycoprotein matrix forms, termed the zona pellucida, separating the oocyte from the GC. The zona pellucida is essential for normal fertilization (Edson et al., 2009). Throughout follicular growth, the GC secrete fluid which gradually coalesces to form an antral cavity during the late secondary stage of growth (Edwards, 1974). This fluid-filled antrum leads to the differentiation of two distinct subpopulations of GC: the cumulus cells (CC) and the mural GC (Figure 1-1 and Figure 1-2). 


\section{Follicle stage}

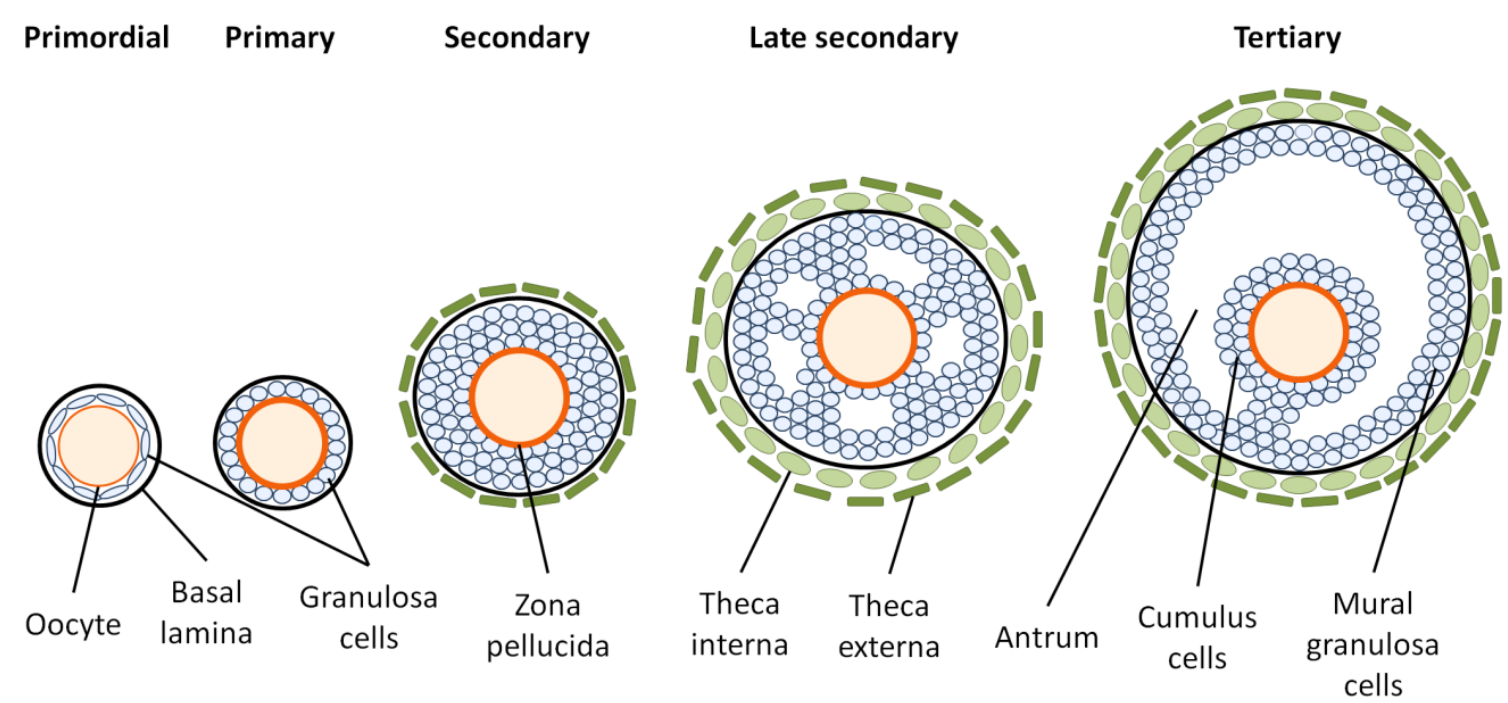

Figure 1-1: The structure of the ovarian follicle during different growth stages. Following initiation of follicle growth, the GC become cuboidal and proliferate to form multiple concentric layers. Secretions from the GC and exudates from the plasma coalesce to form a fluid filled antrum. Concurrently, the GC differentiate into two populations, the mural GC and the CC. The theca interna and theca externa form outside the basal lamina of the follicle. Modified from Johnston, L (VUW BBMedSci Honours thesis, 2010).

The CC directly surround the oocyte, and serve to nurture it (Su et al., 2009). The mural GC line the outer regions of the follicle, perform steroidogenesis and express receptors for pituitary gland-derived gonadotrophins, follicle-stimulating hormone (FSH) and in later follicular stages, luteinising hormone (LH). The differentiation of the mural GC and CC is proposed to be mediated by opposing gradients of FSH from the blood, and factors secreted by the oocyte (Diaz et al., 2007). Following the formation of the antrum and differentiation of the GC, the follicle is termed an antral or tertiary follicle.

During the primary and secondary phases, follicular growth is not reliant on gonadotrophin signalling; conversely tertiary follicles require a functional LH receptor signalling system in theca interna cells and a functional FSH receptor signalling system in GC for continued GC proliferation and synthesis of steroids such as oestradiol (Kumar et al., 1997). Those developing follicles that acquire functional $\mathrm{LH}$ receptors on GC are considered to be the 'dominant' follicles which will go on to be ovulated. The number of follicles that are ovulated during each reproductive cycle depends on the species. Species such as primates (e.g. humans) and ruminants typically ovulate one oocyte per cycle whereas species such as 
rodents and pigs will ovulate multiple oocytes per cycle. These few dominant follicles are the major source of oestrogens and inhibins, which rise cyclically during the mid- to latefollicular stage of the oestrous cycle. Oestrogens and inhibins secreted from the dominant follicle(s) suppress FSH, but not $\mathrm{LH}$, release from the pituitary, thus preventing any follicles that do not have LH receptors expressed on GC from sustaining oestradiol production and further maturation. As FSH is an essential factor for antral follicular development, it is thought a key mechanism to reduce the number of dominant follicles is lowering the plasma concentrations of FSH while increasing the GC responsiveness to $\mathrm{LH}$. This allows maturation of the dominant follicle(s) to continue in increasingly low concentrations of FSH but rising concentrations of LH (Edson et al., 2009).

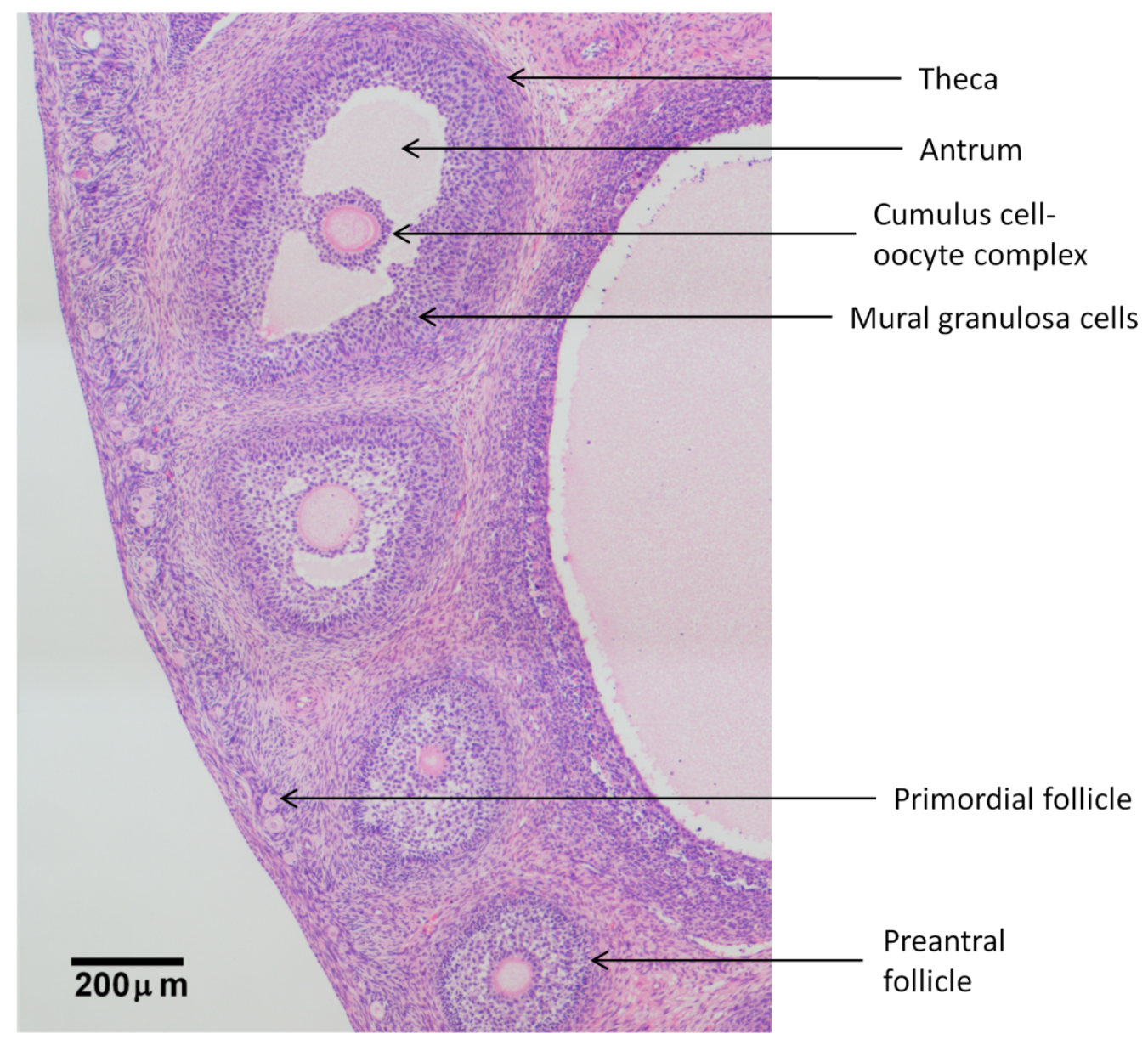

Figure 1-2: Sheep ovarian follicles at various stages of development. A pre-antral follicle with multiple concentric layers of GC may be seen at the bottom of the image. The follicle above this is beginning to form an antrum, while the next follicle has a more developed antrum. The top-most follicle has a large antral space, and the differentiated CC-oocyte complex (COC) is visible in the middle of the follicle. Image supplied by Ken McNatty. 
The final stages of follicular growth involve the maturation of the oocyte nucleus and cytoplasm in preparation for ovulation (Eppig, 1996). Nuclear maturation includes the resumption of meiosis and will be discussed in Section 1.1.3. Cytoplasmic maturation prepares the oocyte for activation and preimplantation development and involves the storage of messenger ribonucleic acid (mRNA) transcripts and proteins for use after fertilisation. These mRNA stores are essential as the embryonic genome is not activated until a few days after fertilisation (Gosden and Lee, 2010). During cytoplasmic maturation, ultrastructural changes also occur including redistribution of the mitochondria, endoplasmic reticulum, and cortical granules (Marteil et al., 2009).

\subsubsection{Oocyte meiotic arrest}

From the time of follicular formation until the pre-ovulatory LH surge, the oocyte is arrested at the diplotene stage of the first meiotic prophase. During this time the DNA is contained within the nuclear envelope, termed the germinal vesicle (GV). The oocyte gains the ability to progress through meiosis around the same time as the antrum forms, although meiotic arrest is maintained until the preovulatory LH surge (Mehlmann, 2005). Resumption of oocyte meiosis can be recognised morphologically by the breakdown of the GV (GVBD). Meiotic progression results in release of the first polar body, but arrests again at metaphase II and only resumes at fertilization. Meiosis is completed following fertilization and the release of the second polar body (Edson et al., 2009)

Meiotic arrest has long been known to be regulated by high levels of cyclic adenosine monophosphate (CAMP) in the oocyte (Cho et al., 1974). Removal of the CC-oocyte complex (COC) from the follicle, or inhibition of gap junction (GJ) transfer, causes cAMP levels within the oocyte to decline, and the oocyte to resume meiosis (Pincus and Enzmann, 1935; Racowsky et al., 1989; Tornell et al., 1990). Within the oocyte, cAMP is synthesised by adenylyl cyclase which is stimulated by a constitutively activated $\mathrm{G}$ protein coupled receptor (Norris et al., 2009). Despite the presence of adenylyl cyclase (Conti et al., 2012), it has been shown that the oocyte receives much of its CAMP from the somatic cells via GJs (Thomas et al., 2002; Webb et al., 2002). Thus, it is understood that intra-oocyte cAMP levels are the net result of a supply of this second messenger from CC via GJ, synthesis within the oocyte itself and the rate of enzymatic degradation. The degradation (hydrolysis) of cAMP occurs mainly by phosphodiesterases (PDEs). The major PDE isoform reportedly expressed in mammalian 
oocytes is PDE3A, and this has been shown to prevent oocyte nuclear maturation both in vitro and in vivo (Conti et al., 2002). While PDE3A preferentially and rapidly hydrolyses CAMP, it also hydrolyses another secondary messenger (cyclic guanosine monophosphate; cGMP), but at a much slower rate. Thus the presence of cGMP within the oocyte slows the hydrolysis of CAMP and is described as an inhibitor of CAMP degradation by PDE3A (Beavo, 1995). During meiotic arrest, CGMP is transferred to the oocyte from the CC via GJs, resulting in a reduction of cAMP hydrolysis by PDE3A (Norris et al., 2009).

One way through which FSH contributes to the maintenance of meiotic arrest is stimulation of the cGMP pathway. Studies in the rat using equine chorionic gonadotrophin reveal that activation of the FSH receptor stimulates mRNA expression of GC-derived natriuretic peptide precursor type C (Nppc), and its receptor on CC (Npr2). The action of NPPC on NPR2 results in an increase in CGMP production by the GC and CC (Zhang et al., 2010). In addition, oestradiol increases the levels of Npr2 mRNA and maintains the ability of NPPC to stimulate cGMP production in vitro (Zhang et al., 2011). Conversely, treatment of mouse follicles with LH caused a rapid reduction in NPR2 guanylyl cyclase activity, without reducing NPR2 protein levels (Robinson et al., 2012). The preovulatory LH surge also triggers closure of the GJs as a result of mitogen activated kinase (MAPK)-dependent phosphorylation of the GJ protein connexin 43 (CX43) (Norris et al., 2008). The net result of NPR2 inhibition and closure of the GJs is that both cAMP and cGMP concentrations within the oocyte decrease and meiosis resumes.

As discussed earlier, oocyte maturation in response to the preovulatory LH surge is a cAMPmediated process. However, CAMP has paradoxical actions in the CC and oocyte. Following the LH surge, a transient rise in cAMP levels occurs in the GC. In the rat, elevated levels of CAMP levels in the follicular cells as a result of inhibition of PDE4 promotes oocyte maturation in the absence of LH (Tsafriri et al., 1996). PDE4D is the major PDE isoform expressed in the CC and GC in rat follicles and is CAMP specific (Tsafriri et al., 1996). PDE4D regulation of $\mathrm{LH}$ receptor signalling is essential, as demonstrated by a PDE4D knockout (KO) mouse model. Following human chorionic gonadotrophin (hCG) stimulation, follicles from KO mice showed an abnormal pattern of cAMP accumulation and failed to rupture, resulting in a decreased ovulation rate (Park et al., 2003). 
In addition to PDE3 and 4, PDE1, 5, 7, 8, 10, and 11 have also been detected in follicles from various species (Sasseville et al., 2009a; Conti, 2011). However, the role of these PDE families in oocyte maturation is less well known and is thought to be secondary to the actions of PDE3/PDE4 in most species. The exception to this is PDE8, which accounts for around $20 \%$ and $60 \%$ of PDE activity in the bovine oocyte and CC, respectively (Sasseville et al., 2009a).

Detection of DNA damage and replication errors during meiosis is critical for maintaining genetic integrity. Two protein kinases that detect DNA damage and regulate progression of meiosis have been identified. The ataxia telangiectasia mutated (Atm) gene is activated by ionizing radiation and double-strand breaks. The ataxia telangiectasia and Rad3-related (Atr) gene is primarily activated by UV light, hydroxyurea, stalled replication forks and singlestranded DNA, but may also be activated by ATM in response to double-strand breaks (Rhind, 2009). Activation of the ATR protein causes the phosphorylation of multiple proteins including CHK1, BRCA1, and NBS1 and results in the formation of check point complexes and $\mathrm{G}_{2} / \mathrm{M}$ arrest (Abraham, 2001; Miles et al., 2010). ATR is localised at unsynapsed chromosomes during the pachytene stage of prophase in human oocytes (Garcia-Cruz et al., 2009). Pachytene is a stage of prophase during which crossing over between homologous chromosomes occurs. The pachytene checkpoint monitors DNA damage, repair of recombination intermediates, and chromosomal synapsis prior to chiasma formation (Kogo et al., 2012). In addition, phosphorylation of ATR/ATM substrates has been detected in bovine oocytes during the second stage of meiosis (MII) (Siemer et al., 2009). Moreover, Chk1 mRNA expression is present in germ cells of female mice at 14.5 days post conception (Hasthorpe et al., 2007) and is essential for maintaining meiotic arrest in Xenopus laevis oocytes (Nakajo et al., 1999).

\subsubsection{The pre-ovulatory LH surge and ovulation}

As follicles grow and synthesise inhibin subunits and oestradiol, levels of inhibins and oestradiol within both the follicular fluid and circulation increase. The high levels of inhibins act on the pituitary gland to inhibit FSH production. Oestradiol production in dominant follicles is driven by the increasing release of LH acting on LH receptors expressed in mural GC of dominant follicles (Edson et al., 2009). The positive feedback effect of oestradiol leads to a preovulatory LH surge. As discussed previously (Section 1.1.3) the LH surge triggers 
oocyte nuclear maturation and resumption of meiosis. In addition, the LH surge initiates morphological changes of the follicle, which are required for ovulation.

The rise in LH levels stimulates the CC to secrete a specialised extracellular matrix, composed of a backbone of hyaluronan in a process termed CC expansion. CC expansion is mediated by MAPK signalling (Su et al., 2002) and oocyte paracrine factors (Buccione et al., 1990b) and results in the $\mathrm{CC}$ transforming from a tightly packed mass to an expanded mucified complex. CC expansion is essential for extrusion of the oocyte from the follicle (Kidder and Vanderhyden, 2010). The follicle wall is also remodelled through capillary haemorrhage, proteolytic degradation and cell death at the apical site and luteinisation of the GC. These events culminate in follicular rupture thereby allowing the expanded $\mathrm{COC}$ to be released from the follicle for entry into the reproductive tract (Russell and Robker, 2007).

The preovulatory LH surge also stimulates luteinisation of the mural GC. Following ovulation and release of the expanded $\mathrm{COC}$, the luteinised GC and, in some species, the theca cells differentiate from the remnants of the haemorrhagic follicle to form the corpus luteum (CL). The $\mathrm{CL}$ is a specialised structure that in all species produces progesterone (and in some species oestradiol and inhibin) to stimulate the uterus to prepare for pregnancy (Edson et al., 2009).

\subsection{The Role of GJs in Folliculogenesis}

\subsubsection{General GJ structure and function}

GJs are intercellular channels that allow direct passage of small molecules $(<1 \mathrm{kDa})$ between the cytoplasm of two adjacent cells. Cells share ions, small metabolites, and second messengers through GJs as a means of intercellular signalling (Bruzzone et al., 1996). GJs are composed of integral membrane proteins (connexins; $C X$ ), which form hexameric pore structures termed hemichannels or connexons (Figure 1-3). The docking of two hemichannels results in formation of a GJ channel.

There are around 20 different connexin proteins encoded in the mammalian genome. Most cells express multiple connexin isoforms, and each connexin isoform has a characteristic tissue expression pattern (Goodenough and Paul, 2009). Connexins are named according to their size; the smallest connexin is CX26 (26 kDa) while the largest connexin is CX57 (57 kDa). 


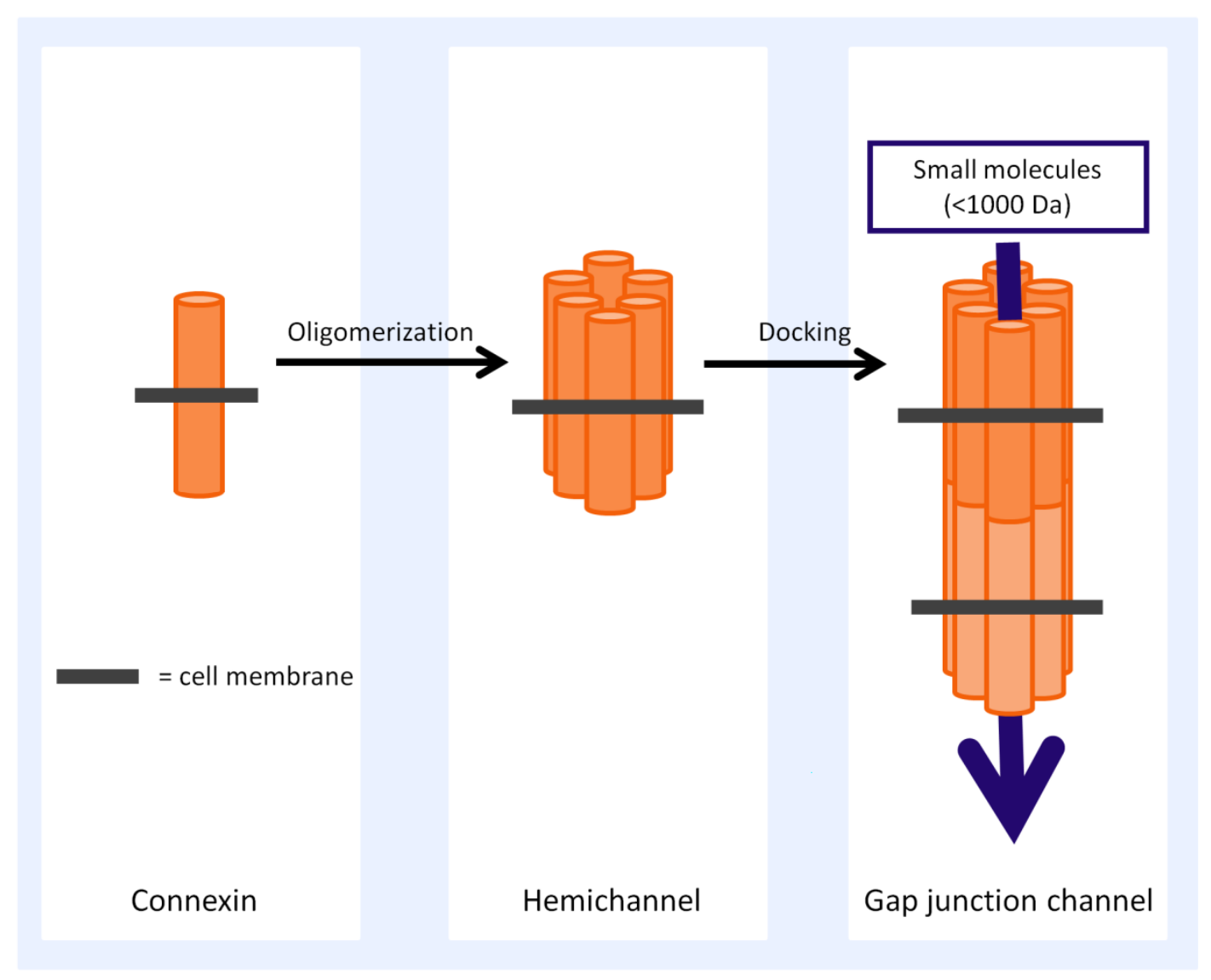

Figure 1-3: Basic structure of GJ channels. CX proteins are transmembrane proteins that form hexameric pore structures (hemichannels). The docking of two hemichannels from adjacent cells forms a GJ channel through which small molecules can pass between the cells.

All connexins have the same structure consisting of a cytoplasmic $\mathrm{C}$ terminus, four membrane spanning domains, two extracellular loops, an intracellular loop, and a cytoplasmic $\mathrm{N}$ terminus (Figure 1-4).

Oligomerization of six connexins to form a hemichannel occurs in the endoplasmic reticulum and Golgi body (Musil and Goodenough, 1993). The hemichannels are transported in vesicles which move along microtubules to the plasma membrane. The vesicles fuse with the plasma membrane, thus delivering the hemichannels to the cell surface (Gaietta et al., 2002; Segretain and Falk, 2004). Analysis of the crystal structure of human CX26 GJs has shown that the hemichannels possess a positively charged cytoplasmic opening, a narrow funnel, a negatively charged transmembrane region and an extracellular exit (Maeda et al., 2009). A large amount of variation in GJs occurs through the different composition of hemichannels. Hemichannels may contain only one connexin isoform or multiple connexin isoforms; these 


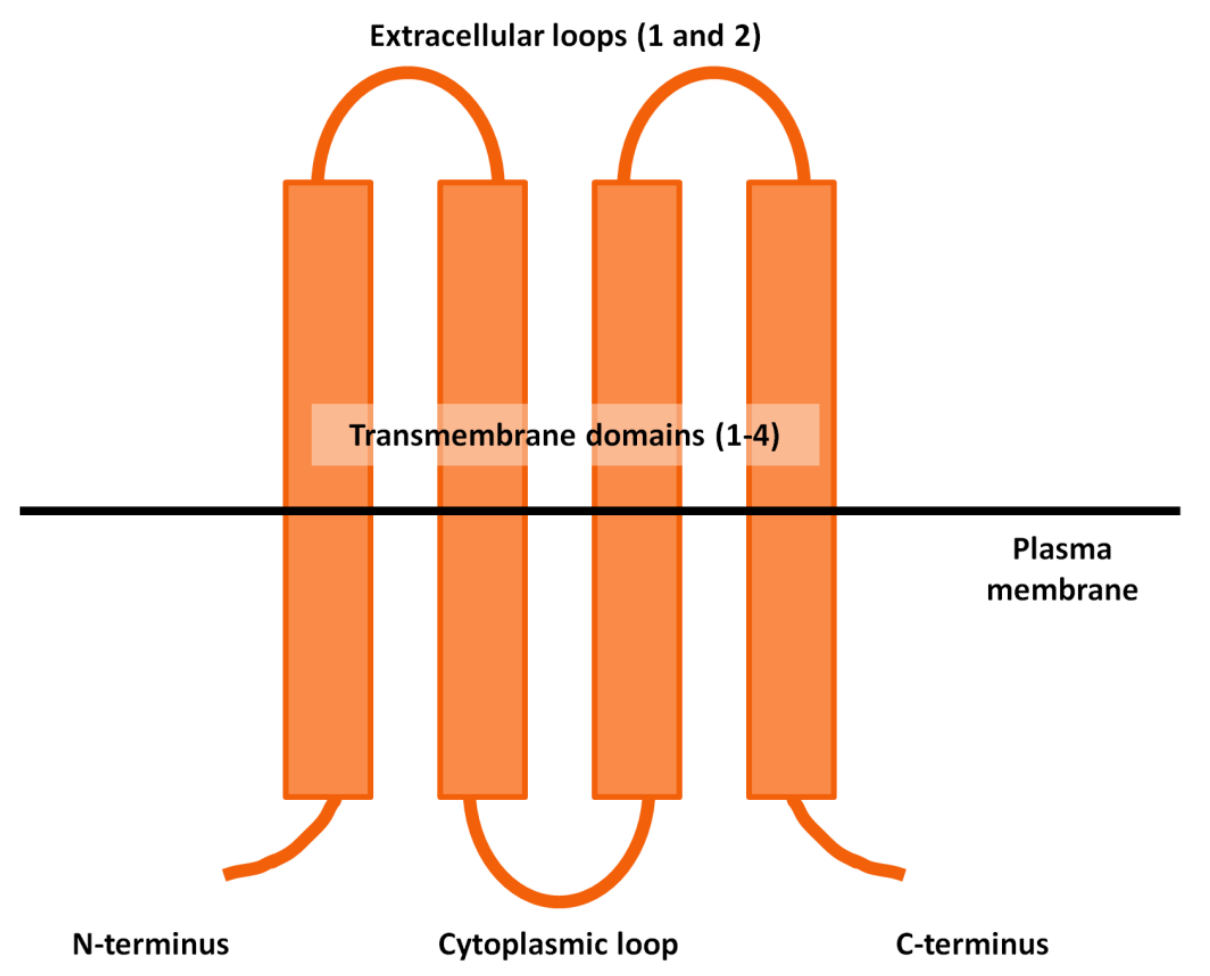

Figure 1-4: Structure of connexin proteins. Connexins are transmembrane proteins which have four transmembrane domains, two extracellular loops, one cytoplasmic loop, and cytoplasmic C- and N-termini. Figure adapted from Kidder and Mhawi, 2002; Maeda et al., 2009.

are termed homomeric and heteromeric hemichannels respectively. It is thought homomeric and heteromeric hemichannels have different permeability to small molecules. Although unpaired hemichannels were previously thought to be non-functional, they have recently been found to play a role in extracellular release of adenosine triphosphate (ATP), Nicotinamide adenine dinucleotide $\left(\mathrm{NAD}^{+}\right)$, prostaglandins, and glutamate, and transduction of $\mathrm{Ca}^{2+}$ waves (Tong et al., 2007).

Two hemichannels from adjacent cells dock through interactions between cysteine residues in the extracellular loops (Dahl et al., 1991). The two hemichannels that make up a GJ channel may be identical or non-identical, forming homotypic or heterotypic GJs respectively (Goodenough and Paul, 2009). The permeability of homotypic GJ channels to small molecules varies greatly. For example, ethidium bromide does not pass through GJs composed of CX31 or CX32, but will pass through GJs composed of CX26, CX37, CX40, CX43, 
or CX45. Moreover, total conductance of GJ channels in cells transfected with CX31 or CX45 is only around $50 \%$ of that observed in cells transfected with other isoforms (Elfgang et al., 1995). Less information is available about the permeability of heterotypic GJ channels due to their complexity, but it is likely that different combinations of connexins give rise to GJ channels with different physiological properties, in a similar manner as that described for homotypic channels (Goodenough and Paul, 2009).

GJs are further assembled into large GJ plaques, which may contain several to thousands of GJ channels. Formation of GJ plaques may be essential for GJ function, as CX43 hemichannels that are not part of a plaque are unlikely to form GJs, and those GJs that are formed are not functional. However, within a GJ plaque composed of CX43 GJs only 2-20\% of the GJs are functional, depending on the size of the plaque (Bukauskas et al., 2000).

GJ permeability is also modified in response to cytoplasm acidification and changes in $\mathrm{Ca}^{2+}$ concentration however these modifications are slower than voltage gating. Increases in $\mathrm{H}^{+}$ and $\mathrm{Ca}^{2+}$ ion concentrations have been demonstrated to reduce the permeability of almost all GJs (Peracchia, 2004). A number of other compounds that specifically inhibit GJs have been identified. These compounds are termed GJ uncouplers and include glyccerrhetinic acid derivatives, long-chain alkanols, and acidifiers (Bukauskas and Verselis, 2004). Inhibition of GJs by GJ uncouplers is reversible and is thought to reduce conductance to the residual state in a similar manner to voltage gating.

GJs are regulated by phosphorylation. All connexins, with the exception of CX26, are phosphoproteins (Ahmad and Evans, 2002; Moreno, 2005). Multiple phosphorylation steps regulate GJ assembly. Connexins are phosphorylated on specific residues during transport through the Golgi, and these phosphorylation sites appear to be essential for trafficking (Solan and Lampe, 2007). Phosphorylation can also inhibit or stimulate connexin degradation (Goodenough and Paul, 2009). Phosphorylation of CX43 at serine (S)368 is thought to prevent CX43 from being assembled into GJs, and results in a $50 \%$ decrease in unitary conductance (Lampe et al., 2000). CX43, the best studied of the connexin isoforms, contains two serines within its $\mathrm{N}$-terminal region and 21 serines within the $\mathrm{C}$-terminal cytoplasmic region (Lampe and Lau, 2000). 
GJs are regulated by rapid turnover: the half-life of CX43 in most cell types is $<3$ hours. Indeed, most connexin isoforms have a half-life of less than 10 hours (Hervé et al., 2007). Turnover of GJs occurs by removal of the channels at the centre of a plaque, and additionally at the edges (Gaietta et al., 2002). Moreover, GJs may be removed from the cell surface by internalisation of the entire GJ plaque (Jordan et al., 2001).

\subsubsection{The Role of GJs in Folliculogenesis}

The follicle functions as a syncytium through folliculogenesis, with the oocyte coupled to the surrounding somatic cells by GJ channels. In a tertiary follicle, the oocyte is coupled to the CC, that are then coupled to the mural GC by GJ channels (Gilula et al., 1978). GJs allow intercellular signalling between the oocyte and somatic cells and provide a pathway for the CC to supply the oocyte with essential nutrients. Glucose metabolites, amino acids, cyclic nucleotides, ions, choline, and inositol are all passed to the oocyte through GJ channels (Kidder and Vanderhyden, 2010). In addition, the GC regulate the $\mathrm{pH}$ of the growing oocyte by transferring ions to the oocyte via GJs (FitzHarris et al., 2007). The GJ channels between the oocyte and CC occur at the end of CC trans-zonal projections (Kidder and Mhawi, 2002).

The major connexin isoforms expressed in rat ovarian follicles are CX37 (expressed in the oocyte) and CX43 (expressed in CC and GC) (Teilmann, 2005). In addition, CX30.3, CX32 and CX45 are expressed in lower proportions in rat GC. Whilst CX57 has been detected in mouse CC, it has not been measured in rat CC (Kidder and Mhawi, 2002).

The essential role of CX37 in follicular growth has been demonstrated by targeted gene KO studies. As CX37 is the major connexin expressed in the oocyte, oocyte-GC coupling is abolished in mice that have a KO of the $C \times 37$ (alternatively named Gja4) gene that encodes CX37. Growth of follicles initially appears to proceed normally in CX37-KO mice however halts at the preantral stage (Simon et al., 1997). The size of oocytes from these mice reached only $74 \%$ of that of fully grown oocytes from wild-type mice and did not gain meiotic competence (Carabatsos et al., 2000). The ovaries of $C \times 37-\mathrm{KO}$ mice eventually become filled with CL-like structures, indicating premature differentiation of the GC into luteal cells (Simon et al., 1997). Expression of $C \times 37$ mRNA in oocytes, but not GC, is essential for follicle growth as demonstrated by the use of chimeric ovaries containing wild-type or CX37-KO oocytes and wild-type or Cx37-KO GC in all combinations (Gittens and Kidder, 2005). Chimeric ovaries 
were created in vitro and implanted in the kidney capsule of ovariectomized female wildtype mice. Ovaries containing wild-type oocytes and Cx37-KO GC showed similar proportions of follicles at each stage of development compared to ovaries containing wild-type oocytes and wild-type GC. However, ovaries containing Cx37-KO oocytes and wild-type GC had significantly reduced proportions of late secondary and tertiary follicles compared to completely wild-type ovaries.

Due to the expression of CX43 in many tissues, mice which have a targeted KO of $C_{x 43}$ (alternatively named Gja1) gene are aborted in utero. In order to study the role of CX43 in follicular growth, Cx43-KO ovaries from neonatal mice were cultured in vitro before being grafted into the kidney capsule of ovariectomized wild-type adult mice (Ackert et al., 2001). GC from Cx43-KO mice were unable to proliferate causing follicular growth to arrest at the primary stage. Oocytes from Cx43-KO ovaries were morphologically abnormal, did not gain meiotic competence and could not be fertilised. Dye transfer experiments indicated that some GJ channels were retained between the GC, consistent with expression of other connexin isoforms. The essential role of GC, but not oocyte, expression of CX43 was demonstrated using chimeric ovaries containing wild-type or Cx43-KO oocytes, and wild-type or Cx43-KO GC in all combinations (Gittens and Kidder, 2005). Ovaries with CX43-KO oocytes and wild-type GC contained follicles at all developmental stages in similar proportions to those seen in ovaries containing wild-type oocytes and wild-type GC. Ovaries with wild-type oocytes and Cx43-KO GC had significantly reduced proportions of early secondary, late secondary and tertiary follicles compared with the wild-type comparison, consistent with a block at the primary stage of follicular development.

As Cx37 mRNA is expressed solely by the oocyte and CX43 mRNA is expressed in the CC and GC, the GJs that form between the oocyte and CC must be heterotypic. Conductance studies of cell lines transfected with rat $C \times 43$ and/or human $C \times 37$ genes showed that homotypic, heterotypic, and heteromeric GJs are able to be formed in vitro (Brink et al., 1997). However, to my knowledge this has not been demonstrated in vivo.

Within the follicle, Cx43 mRNA and protein levels are dynamically regulated throughout the oestrus cycle by gonadotrophins. In antral follicles of rats that express FSH receptors, rising levels of FSH stimulate an increase in CX43 mRNA and protein levels (Granot and Dekel, 
1997). As discussed previously (Section 1.1.3), cAMP and cGMP pass through the GJ to the oocyte to maintain the threshold concentration of CAMP that inhibits meiotic resumption. The FSH-mediated increase in oocyte cAMP is blocked by treatment with the GJ uncoupler carbenoxolone (Webb et al., 2002) confirming that cAMP is transferred to the oocyte from the CC via GJ. Following the LH surge in mice, the decrease in GJ permeability between the oocyte and CC is mediated by the phosphorylation of CX43 at S255, S262, and S279/S282 (Norris et al., 2008). Subsequently, Cx43 mRNA expression is abolished (Granot and Dekel, 1997). To my knowledge, $C \times 37$ gene expression has not been reported to be regulated by gonadotrophins.

The CC transfer a range of nutrients to the oocyte via GJs. Compared with denuded oocytes (DO), CC-enclosed mouse oocytes had a significantly higher uptake of $\left[{ }^{3} \mathrm{H}\right]$ glycine and $\left[{ }^{3} \mathrm{H}\right]$ choline, however this was abolished following treatment with the GJ uncoupler $18-\alpha-$ glycyrrhetinic acid (Haghighat and Van Winkle, 1990). Glucose and glucose metabolites are transferred into the oocyte from the CC via GJs. The amount of a fluorescently-labelled glucose derivative in the mouse oocyte was significantly reduced when the GJs were blocked with the GJ uncoupler carbenoxolone (Wang et al., 2012). CC-enclosed mouse oocytes also contain higher amounts of amino acids glycine, alanine, proline, serine, tyrosine, glutamic acid, and lysine compared with DO (Colonna and Mangia, 1983). Moreover, $\mathrm{Ca}^{2+}$ and $\mathrm{Na}^{+}$ ionic currents between mouse CC and oocytes were abolished following treatment with the GJ uncoupler octanol (Arellano et al., 2002).

In addition to fully-formed GJ channels, undocked hemichannels may play a role in ovarian folliculogenesis. Dye uptake and ATP release, indicative of hemichannel opening, was observed in mouse GC following application of stimuli known to cause hemichannel opening (Tong et al., 2007). However hemichannel function in the absence of GJs is not sufficient for follicle growth. Reaggregated ovaries containing GC with a mutant $C_{x} 43$ gene that lacked the conserved cysteines in the extracellular loops required for docking were created. Follicle growth was arrested at the primary stage, similar to $\mathrm{C} \times 43-\mathrm{KO}$ mutants. 


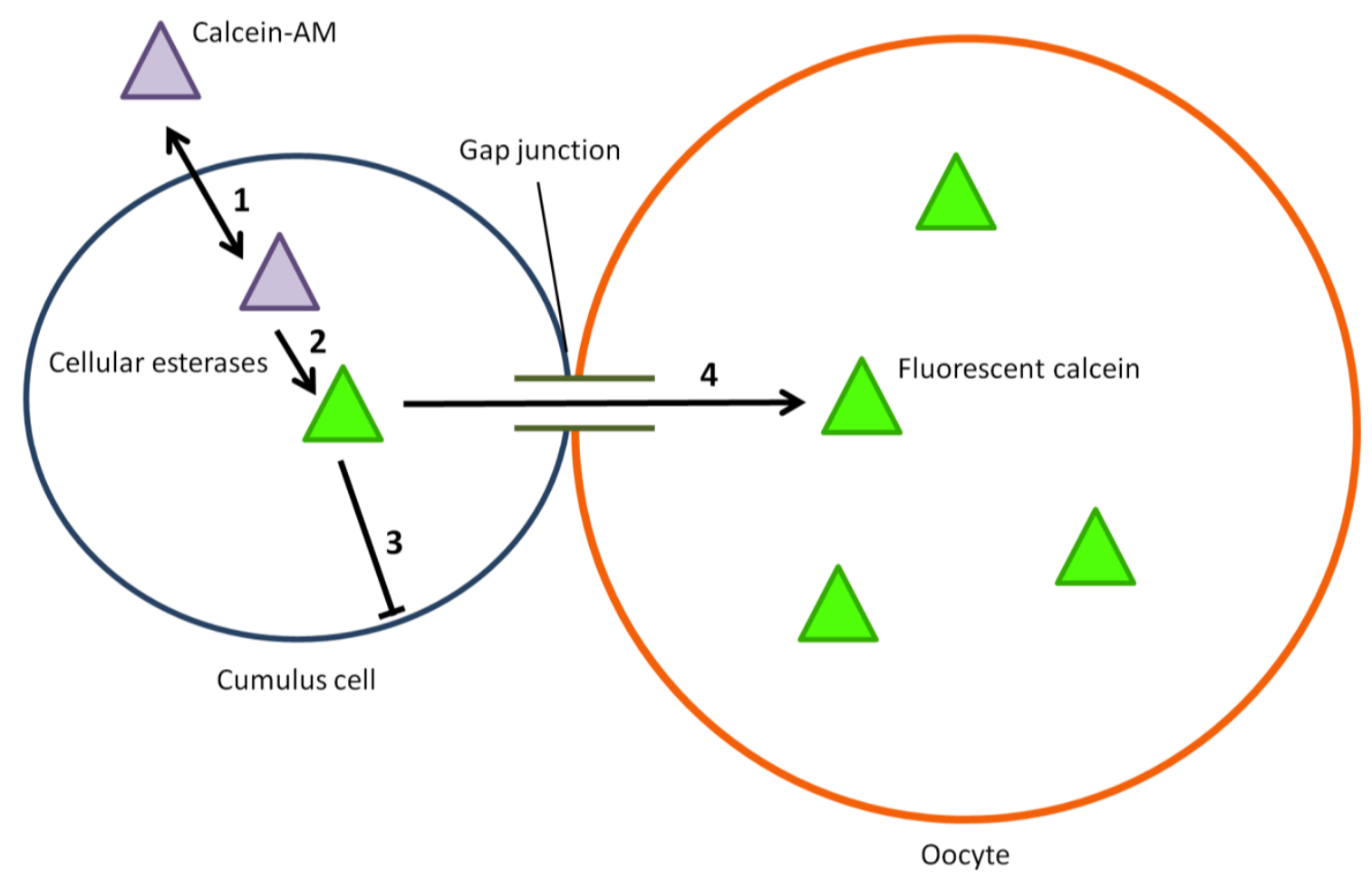

Figure 1-5: Schematic of a GJ bioassay utilising calcein to measure GJ communication between the CC and oocyte. Calcein-AM enters the CC by permeating the cell membrane (1). Inside the CC, the AM group is cleaved by non-specific esterases (2). The resultant calcein molecule is lipid insoluble and fluorescent (3). Calcein is transferred to the oocyte through GJ channels (4).

A technique developed to determine GJ activity between the CC and oocyte utilised the transfer of a fluorescent dye, calcein (Thomas et al., 2004). Intact COC were exposed to the acetoxymethyl (AM) derivative of the calcein. Calcein-AM is a non-fluorescent, lipophilic molecule that readily crosses the cell membrane. The AM group is cleaved by non-specific esterases inside the cell. As a consequence of removing the AM moiety, the resultant calcein is now lipid insoluble and hydrophilic. Thus being unable to permeate the cell membrane, calcein can now only be transferred between cells through GJ channels (Figure 1-5).

\subsection{Oocyte-secreted factors}

\subsubsection{Oocyte-secreted factors and follicular development}

During follicular growth, co-ordination between the follicular somatic cells and the oocyte is essential. As discussed in the previous section, the CC transfer nutrients to the oocyte via GJs. The oocyte regulates CC function through secretion of growth factors, thus creating a bidirectional communication loop. Aspiration of the $\mathrm{COC}$ causes the remaining GC to undergo 
premature luteinisation (el-Fouly et al., 1970). Soon after it was found that rat GC cocultured in close proximity to the oocyte retained their GC phenotype whereas GC further away from the oocyte underwent luteinisation (Nekola and Nalbandov, 1971). Further investigation revealed that oocyte-secreted factors form a concentration gradient in the follicle, and this influences the differentiation of GC into either the CC or the mural GC. Compared to mural GC, CC have a higher proliferation rate and lower steroidogenic activity and expression levels of $\mathrm{LH}$ receptor. The CC also possess the ability to secrete hyaluronic acid and undergo expansion, unlike the mural GC (Gilchrist et al., 2004a). The best characterised of the oocyte-secreted factor candidates are growth differentiation factor 9 (GDF9) and bone morphogenetic protein 15 (BMP15). In most species studied to date, GDF9 and BMP15 are co-expressed in a species-specific manner and the two secreted proteins function synergistically (McNatty et al., 2005a; Crawford and McNatty, 2012). Although the addition of GDF9 and BMP15 to cultured GC in part mimics the addition of oocytes, they are not sufficient to produce the range of GC responses caused by oocytes (Gilchrist et al., 2004a). This indicates that other oocyte-secreted factors exist that may play a role in somatic cell function. Alternatively, the recombinant forms of GDF9 and/or BMP15 tested in vitro may have been at inappropriate concentrations or ratios, or different biopotencies to that of native GDF9 and BMP15. Currently, other candidates for oocyte-secreted factors are also members of the TGF $\beta$ family, including BMP6 and the activins.

Bioassays are commonly used to determine the effects of oocyte-secreted factors on GC function. One common method involves removal of the oocyte from the COC to produce oocytectomised (OOX) CC. The basis of this bioassay is to determine the functions of CC that are regulated by oocyte-secreted factors, on the assumption that OOX CC will be significantly altered compared to CC from intact COC. These OOX CC may then be co-cultured with DO which would rescue the CC function (Gilchrist et al., 2008).

The importance of the oocyte on follicular development was demonstrated by transferring mouse oocytes from secondary follicles into primordial follicles. The rate of follicular growth in the re-aggregated follicles was doubled, producing antral follicles within 9 days as opposed to 20 days in control follicles (Eppig et al., 2002). This indicates that the oocyte strongly regulates follicular growth and development, including GC proliferation. Studies involving the culturing of DO with GC revealed oocyte-secreted factors stimulated the 
proliferation rate of GC in many mammalian species including cows (Li et al., 2000; Glister et al., 2003), mice (Vanderhyden, 1992; Gilchrist et al., 2004b) and rats and sheep (McNatty et al., 2005a, 2005b; Lin et al., 2012). In mice, co-culture of mural GC with DO caused an upregulation of the gene encoding cyclin D2 in the GC (Gilchrist et al., 2006). These actions of DO on GC proliferation are due, at least in part, to both GDF9 and BMP15 as is observed in studies in rodents (Otsuka et al., 2000; Vitt et al., 2000, Lin et al., 2012). Immunoneutralization of GDF9 in an in vitro bioassay abolished around half of the mitogenic activity of mouse oocytes, indicating that other oocyte-secreted factors are also involved in stimulation of GC proliferation (Gilchrist et al., 2004b). Importantly, GDF9 and BMP15 act in synergy with one another to stimulate proliferation in rat and ovine GC (McNatty et al., 2006). Addition of recombinant murine GDF9 in combination with ovine BMP15 to rat GC enhanced ${ }^{3} \mathrm{H}$-thymidine uptake by greater than 3-fold compared to either oocyte-secreted factor alone (McNatty et al., 2005a). Addition of recombinant ovine GDF9 in combination with ovine BMP15 to ovine GC enhanced ${ }^{3} \mathrm{H}$-thymidine uptake by 1.6 -fold compared to GC cultured in the absence of oocyte-secreted factors (McNatty et al., 2005b). It should be noted that there are significant species differences in the potency of oocyte-secreted factor action on GC proliferation that are outside the scope of this literature review. Additionally, whilst some oocyte-secreted factors (e.g. murine) strongly stimulate GC proliferation in vitro (Gilchrist et al., 2001), oocyte-secreted factors from other species (e.g. bovine and porcine) require the addition of insulin-like growth factor 1 (Li et al., 2000; Hickey et al., 2005).

In addition to the effects of oocyte-secreted factors on GC proliferation, oocyte-secreted factors have also been reported to reduce luteinisation of CC. Relatively high expression levels of the LH receptor and high steroidogenic activity are observed in mural GC from intact human and bovine follicles compared to CC (Amsterdam et al., 1975; Li et al., 2000). Evidence for the involvement of oocyte-secreted factors in regulating somatic cell luteinisation is further strengthened by the observation that progesterone production and LH receptor expression are increased in mouse OOX CC compared to either intact COC or OOX CC cultured with DO (Eppig et al., 1997). Both BMP15 and GDF9 are inhibitors of CC luteinisation in rats. The action of BMP15 alone does not affect steroidogenesis, but inhibits FSH action thereby abolishing FSH-mediated progesterone production and upregulation of the LH receptor and other key receptors involved in luteinisation (Otsuka et al., 2001). GDF9 
inhibits the stimulatory effect of FSH on oestradiol and progesterone production, LH receptor activity, and cAMP production by GC (Vitt et al., 2000). Both GDF9 and BMP15 cooperate to inhibit luteinisation of rat and bovine GC (McNatty et al., 2006). In both of these studies, the combined addition of recombinant ovine GDF9 and BMP15 resulted in reduced progesterone production compared to either GDF9 or BMP15 alone.

As discussed in Section 1.1.4, CC expansion occurs just prior to ovulation in response to unidentified oocyte paracrine factors (termed the CC expansion enabling factor; CEEF) and the preovulatory LH surge. In mice, expansion of CC can be prevented by removal of the oocyte or inhibition of oocyte signalling, and can subsequently be restored by co-culturing CC with DO (Buccione et al., 1990a). Though the identity of the CEEF is currently unknown, GDF9 has long been suspected as the primary candidate. This is supported by the findings that GDF9 stimulates CC expansion in vitro (Elvin et al., 1999) and that DO from GDF9 KO mice were unable to stimulate CC expansion in wild-type CC. However, there is evidence negating that GDF9 alone is sufficient to induce CC expansion. For example, immunoneutralization of GDF9 did not inhibit oocyte stimulated CC expansion (Dragovic et al., 2005). Moreover, meiotically incompetent oocytes from small growing follicles express GDF9 (Juengel and McNatty, 2005) but are not able to stimulate CC expansion (Vanderhyden et al., 1990). OOX CC isolated from BMP15 $5^{-/} \mathrm{GDF}^{+/-}$double mouse mutants had a reduced ability to undergo expansion compared to either the single mutants (i.e. BMP15 $15^{-/-} \mathrm{GDF}^{+/+}$or $\mathrm{BMP} 15^{+/+} \mathrm{GDF}^{+/-}$) (Su et al., 2004). Taken together, these results suggest that while GDF9 may partially account for the ability of the oocyte to promote CC expansion, BMP15 and other oocyte factors may also play a role.

In vitro studies have also revealed that in a similar manner to DO, GDF9 and BMP15 significantly prevent CC apoptosis in bovine OOX CC (Hussein et al., 2005). Interestingly however, culturing OOX CC with GDF9 alone had no effect on CC apoptosis. The action of GDF9 has also been reported on follicular constituents additional to CC. The addition of GDF9 to cultured rat GC stimulates the production of inhibin A and inhibin B (Roh et al., 2003). It has also been reported to regulate theca cell function, as recombinant GDF9 strongly stimulated basal and forskolin-stimulated androstenedione production in theca cells in vitro (Solovyeva et al., 2000). 
The regulation of GC function by the oocyte varies during folliculogenesis. The ability of oocytes to stimulate GC proliferation decreases as the oocyte matures. Thymidine uptake in murine GC was greatest when the GC were co-cultured with oocytes that had a visible GV. Although maturing and ovulated oocytes (i.e. those that had initiated GVBD) were also able to stimulate thymidine uptake in GC, the effect was attenuated (Gilchrist et al., 2001). Conversely, the ability of the oocyte to inhibit progesterone production in GC increases throughout maturation. Progesterone production was maximally inhibited when murine OOX CC were co-cultured with fully-grown oocytes rather than growing oocytes. However, CC from ovulated $\mathrm{COC}$ produced large amounts of progesterone due to a reduced ability to respond to the oocyte-secreted factors (Vanderhyden and Macdonald, 1998). Both LH receptor mRNA expression and CC-responsiveness to FSH and CEEF are developmentally regulated by oocyte-secreted factors in mice in a similar manner to progesterone production (Vanderhyden et al., 1990; Eppig et al., 1997).

\subsubsection{Oocyte-secreted factors and follicular metabolic functions}

Fully-grown bovine and rat oocytes have a poor capacity for glucose metabolism, yet are completely reliant on oxidative phosphorylation for ATP production (Zeilmaker and Verhamme, 1974; Cetica et al., 2002). In large antral follicles, CC readily import and metabolise glucose, and transfer glucose metabolites to the oocyte for ATP production (Brower and Shultz, 1982; Cetica et al., 2002). The expression levels of enzymes involved in glucose breakdown, including phosphofructokinase (Pfkp), aldolase A (Aldoa), triosephosphate isomerise (Tpi), $\alpha$-enolase (Eno1), pyruvate kinase (Pkm2), and lactate dehydrogenase (Ldh1), showed a morphogenic gradient across the mouse follicle with highest levels being measured in the CC and lowest levels in the outer mural GC (Sugiura et al., 2005). Gene expression levels of Eno1, Pkm2, Ldh1, and Pfkp were significantly downregulated in OOX CC compared with intact $\mathrm{COC}$ in mice. However, expression levels were restored to those seen in intact $\mathrm{COC}$ when OOX CC were cultured with fully grown oocytes. Similarly, glycolytic activity and tricarboxylic acid (TCA) cycle activity in mice were equivalent in intact $\mathrm{COC}$ and $\mathrm{OOX}$ CC cultured with fully grown oocytes but were downregulated in OOX CC.

These results demonstrate the potent effect that oocyte-secreted factors have on CC glucose metabolism. Importantly however, there are significant species differences in the ability of 
oocyte-secreted factors to regulate CC glycolytic activity. For example, bovine COC, OOX, and OOX co-cultured with DO showed similar glucose consumption in vitro(Sutton et al., 2003). Conversely, mouse OOX have a 10 -fold decrease in glycolytic activity and reduced expression of Pfkp, and Ldh1 compared to COC and OOX co-cultured with DO (Sugiura et al., 2005). Oocyte-secreted factors are also implicated in the regulation of cholesterol biosynthesis in CC in mice. The expression of enzymes involved in cholesterol biosynthesis, including mevalonate kinase $(M v k)$, phosphomevalonate kinase (Pmvk), farnesyl disphosphate synthase (Fdps), squalene epoxidase (Sqle), cytochrome p450 51 (Cyp51), sterol-C4-methyl oxidase-like ( $\mathrm{Sc} 4 \mathrm{~mol})$, and $3 \beta$-hydroxysteroid- $\Delta 8, \Delta 7$-isomerase $(E b p)$ were elevated in CC of COC, compared to OOX CC (Su et al., 2008). Similar to the glycolytic pathway, GDF9 and BMP15 also regulate cholesterol biosynthesis. In Bmp15 and Gdf9 double KO mutants, lower levels of enzymes involved in cholesterol biosynthesis, (Mvk, Pmvk, Fdps, Sqle, Cyp51, Sc4mol, and Ebp) as well as glycolytic enzymes (Pfkp and Ldh1), were measured in CC. In addition, reduced cholesterol biosynthesis and glycolytic activity was observed in CC from the double mutants (Su et al., 2008). Treatment of double mutant CC with recombinant BMP15 in combination with fibroblast growth factor 8B (FGF8B) promoted expression of Pfkp and Ldh1. As GDF9 did not promote expression of Pfkp and Ldh1, it appears that BMP15 but not GDF9 regulates CC glycolytic activity (Sugiura et al., 2008).

Oocyte-secreted factors have been reported to regulate the uptake of amino acids in CC via the sodium-coupled neutral amino acid transporter Slc38a3 (Eppig et al., 2005). In mice, the SIc38a3 gene is expressed in mural GC and CC of early tertiary follicles, but mural GC expression is abolished in late tertiary follicles. Expression levels of S/c38a3 mRNA and uptake of alanine, histidine, and leucine were similar in intact COC and OOX CC cultured with fully grown oocytes, but were downregulated in OOX CC. Fully-grown, but not growing, oocytes were able to restore S/c38a3 mRNA levels and uptake of alanine and leucine to those observed in intact COC. Interestingly, S/c38a3 mRNA was not affected in Bmp15 or Bmp15/Gdf9 KO CC, indicating that other oocyte-secreted factors are responsible for regulating amino acid uptake (Su et al., 2009).

\subsubsection{GDF9 and BMP15 expression and signalling in the ovary}

Both GDF9 and BMP15 are members of the transforming growth factor $\beta$ (TGF $\beta$ ) superfamily of proteins and are thought to be exclusively expressed in the oocyte of most mammalian 
species (Crawford and McNatty, 2012). GDF9 and BMP15 have been reported to be expressed by the somatic cells in pig, goat, and primates (Duffy, 2003; Silva et al., 2005; Paradis et al., 2009) however it is possible that these samples were contaminated with oocyte mRNA. There are significant species differences in the timing and levels of expression. For example, GDF9 is expressed in oocytes from the primordial stage onwards in cows, sheep, and brushtail possums (Bodensteiner et al., 1999; Eckery et al., 2002). BMP15 is also expressed from the primordial stage in the brushtail possum (Eckery et al., 2002), however is expressed from primary stage in sheep (Galloway et al., 2000). GDF9 and BMP15 are expressed only in growing follicles in mice, rats, and humans (Laitinen et al., 1998; Aaltonen et al., 1999; Jaatinen et al., 1999). Recent work indicates that rats and mice produce very little BMP15, compared to GDF9. The ratio of Gdf9:Bmp15 mRNA was 3.65 and 5.19 in the rat and mouse, respectively (Crawford and McNatty, 2012). Moreover, mature GDF9 protein was detected by Western blotting in rat oocyte lysate and in the oocyte culture medium, indicating secretion of GDF9 (Lin et al., 2012). However, mature BMP15 protein was not detected in rat oocyte lysate or in the culture medium. The promature form of BMP15 was detected in rat oocyte lysate however the biological activity is unknown. Furthermore, immunoneutralization of BMP15 in rat oocytes did not affect the ability of those oocytes to stimulate $\mathrm{GC}{ }^{3} \mathrm{H}$-thymidine uptake indicating BMP15 by rat oocytes does not play a role in regulating GC proliferation.

Unlike most other TGF $\beta$ proteins, GDF9 and BMP15 lack the conserved cysteine residues that are required for covalent dimer formation. It has been reported that GDF9 and BMP15 may form non-covalent homo- and hetero-dimers in vitro (Peng et al., 2013) however the existence of these forms in vivo remains a matter of debate (Mottershead et al., 2013). What is known is that GDF9 and BMP15 act synergistically both in vivo and in vitro to influence somatic cell function (Hanrahan et al., 2004; McNatty et al., 2005a; Mottershead et al., 2012). GDF9 and BMP15 regulate GC function by activating two serine/threonine kinase receptors located on the plasma membrane of GC. Binding of the ligand causes activation of type II receptor kinase activity and phosphorylation of the type I receptor (Figure 1-6). The type I receptor then phosphorylates specific regulatory similar to mothers against decapentaplegic (SMAD) proteins (rSMADs) resulting in the phosphorylated rSMAD binding to the common SMAD4. The rSMAD/SMAD4 complex translocates to the nucleus and 
modulates gene expression through interactions with transcription factors, co-activators, and co-repressors (Knight and Glister, 2006). GDF9 signals through the activin-like receptor kinase (ALK) 5 receptor and BMP receptor type II (BMPRII) and activates the SMAD2/3 pathway (Mazerbourg et al., 2004). BMP15 signals through ALK6 and BMPRII and activates the SMAD1/5/8 pathway (Juengel and McNatty, 2005). The synergistic actions of GDF9 and BMP15 act through the SMAD2/3, but not SMAD1/5/8, pathway (Reader et al., 2011).

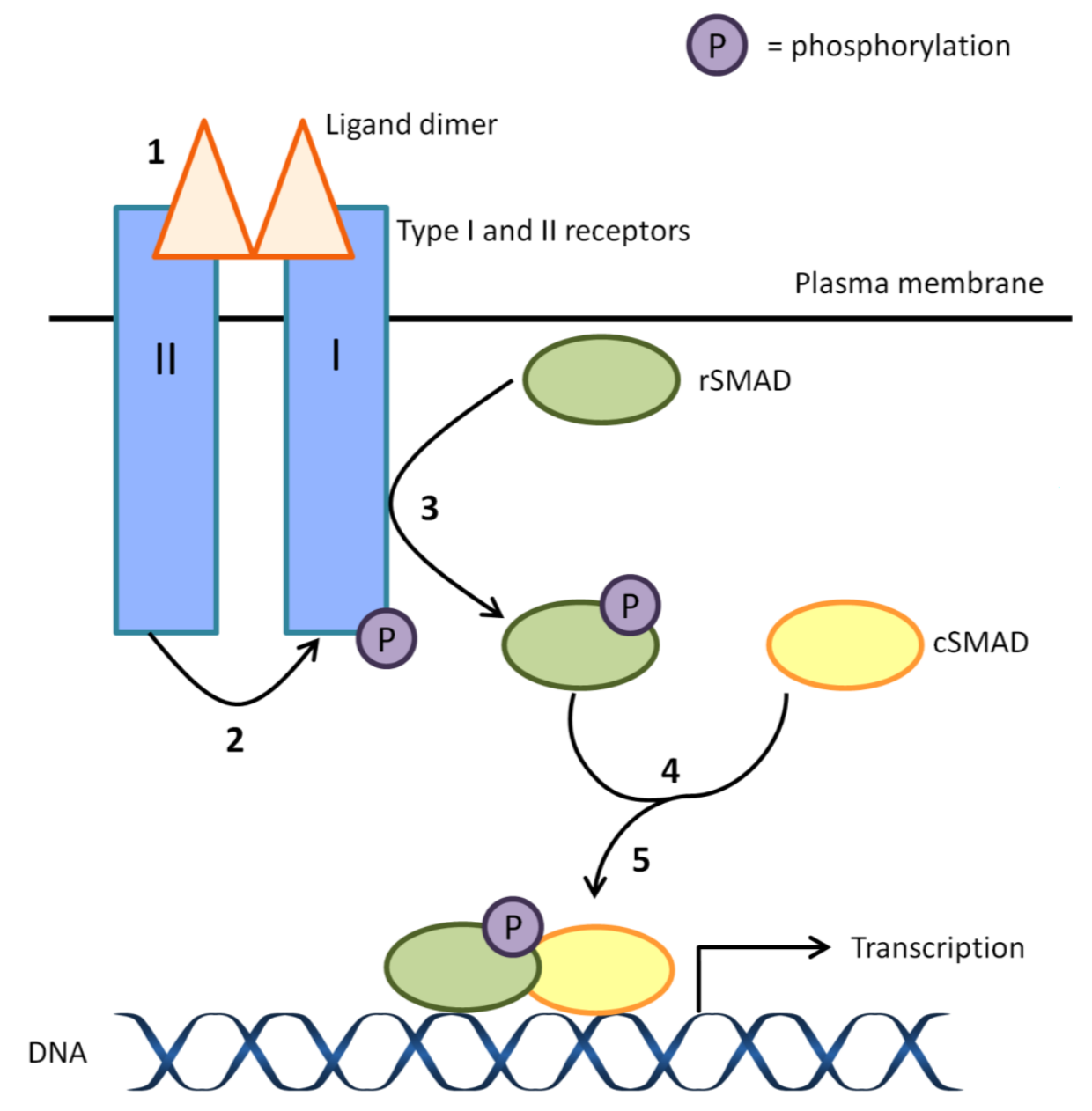

Figure 1-6: Schematic overview of the TGF $\beta$ family signalling pathway. The ligand dimer binds to the Type II receptor (1) which recruits and phosphorylates the Type I receptor (2). The Type I receptor phosphorylates an rSMAD (3), which binds to the common SMAD (4), and this complex translocates to the nucleus where it modulates gene expression (5). 
Whilst GDF9 is required for follicular growth in both sheep and mouse, BMP15 is required for follicular growth in sheep but not mouse. This supports evidence that BMP15 does not play a large role in follicular function of rodents. Follicles from sheep with a naturallyoccurring homozygous $\mathrm{Bmp}^{-5^{--}}$or $\mathrm{Gdf9} \mathrm{F}^{--}$mutation arrest growth at the primary stage, causing sterility (Hanrahan et al., 2004). Interestingly, sheep heterozygous for mutations in either $\mathrm{Gdfg}^{-/+}$or $\mathrm{Bmp}^{-/+}$have a higher ovulation rate than wildtype animals (Galloway et al., 2000; Hanrahan et al., 2004). Conversely, whilst follicular development arrests after the primary follicle stage in Gdf9 KO mice, mice with a Bmp15 KO are fertile, although produce smaller litters (Yan et al., 2001). Mice heterozygous for the Gdf9 KO showed normal fertility (Dong et al., 1996).

\subsection{The Role of Glucose Metabolism}

\subsubsection{Overview of glucose metabolic pathways}

Glucose may be metabolised by several different pathways, each playing a different function within the cell. The primary pathways described herein are those concerned with glycolysis and hexosamine biosynthesis, with brief descriptions of the pentose phosphate and the polyol pathways.

Glycolysis functions primarily to generate energy for the cell and involves the conversion of glucose to pyruvate through multiple steps (Figure 1-7). The conversion of fructose 6phosphate to fructose 1,6-bisphosphate by PFKP is considered to be the rate limiting step (Krisher, 2004). Pyruvate may be converted into lactate by the enzyme LDH1 or into acetyl coenzyme $A(C \circ A)$ by the enzyme pyruvate dehydrogenase. Lactate can also be recycled back to glucose by gluconeogenesis, or to pyruvate. Acetyl CoA enters into TCA cycle, which produces ATP and nicotinamide adenine dinucleotide (NADH) which, together with ribose, is important for the reduction of the antioxidant glutathione. The NADH produced in the TCA cycle is utilised for oxidative phosphorylation by the mitochondrial electron transport chain. In addition, glycolysis intermediates are used for a number of other cellular pathways including the pentose phosphate pathway (glucose 6-phosphate) and lipid metabolism (dihydroxyacetone phosphate) (Voet and Voet, 2004). During times of rest, or in response to high glucose levels, glucose 6-phosphate may alternatively be converted into the stored form of glucose, glycogen, by the glycogenesis pathway. 
Oxidative phosphorylation refers to the coupled processes of ATP synthesis and the electron transport chain within the mitochondria. The electron transport chain removes electrons from NADH and succinate, resulting in conversion of oxygen to water concurrent with the transfer of $\mathrm{H}^{+}$across the mitochondrial membrane. This proton-motive force drives ATP synthase resulting in the transfer of adenosine disphosphate (ADP) to ATP. The stepwise removal of electrons from $\mathrm{NADH}$ and succinate involves several electron acceptors, including NADH dehydrogenase, succinate dehydrogenase, ubiquinone, cytochrome $b_{1}$ complex, cytochrome c, and cytochrome c oxidase. Cytochrome $\mathrm{c}$ is localised in the intermembrane space, and is responsible for transferring one electron to cytochrome c oxidase (Voet and Voet, 2004). Cytochrome $\mathrm{c}$ is a common mitochondrial marker, that correlates well to other mitochondrial markers including citrate synthase and respiratory capacity (Fitts et al., 1975). In addition to its role in the electron transport chain, cytochrome c plays an essential role in activation of apoptosis. Mitochondrial activation of the intrinsic apoptotic pathway results in release of cytochrome $c$ into the cytoplasm, where it binds to apoptotic protease activating factor 1 (APAF1) and pro-caspase-9 resulting in activation of effector caspases and initiation of apoptotic processes (Chandra et al., 2002) 


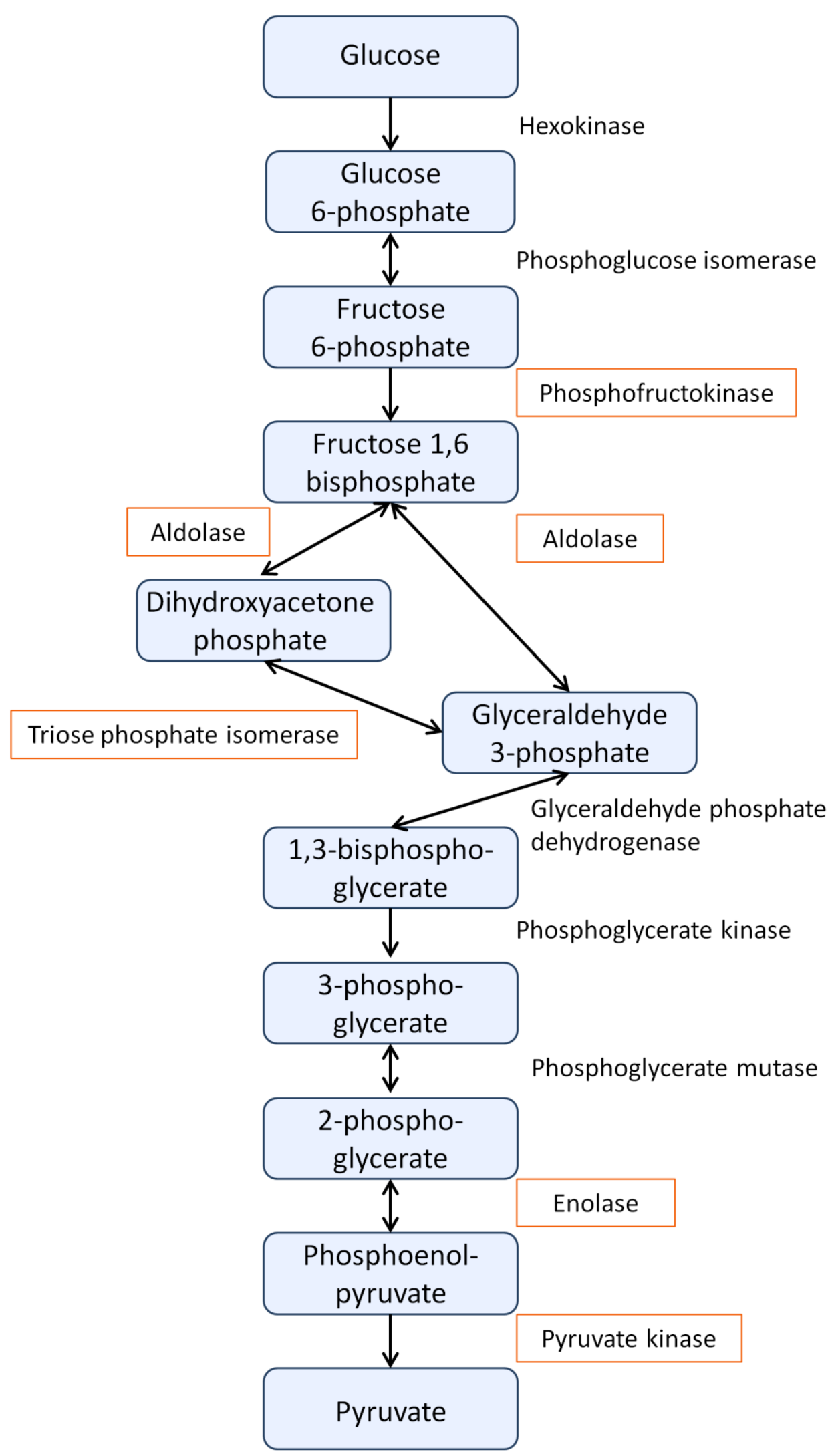

Figure 1-7: The glycolysis metabolic pathway. The blue boxes contain the pathway intermediates/products, while the enzymes that catalyse each reaction are beside the arrows. Those enzymes that are outlined in orange are thought to be regulated by oocyte-secreted factors. 
The hexosamine biosynthetic pathway converts glucose-6-phosphate to uridine disphosphate (UDP)- $N$-acetyl glucosamine (Figure 1-8). The rate limiting enzyme is glutamine-fructose-6-phosphate transaminase 1 (GFPT1). The hexosamine biosynthesis pathway acts as a fuel sensing pathway. Typically, only a small amount of glucose 6phosphate (1-3 \%) is metabolised through this pathway. Increased flux through the hexosamine pathway causes decreased uptake of glucose, and increased glycogen accumulation (Rossetti, 2000). The UDP- $N$-acetyl glucosamine produced by the hexosamine pathway is used for O-linked glycosylation (Sutton-McDowall et al., 2010). The hexosamine pathway plays an additional role in pre-ovulatory follicles, as UDP-N-acetyl glucosamine is a precursor for hyaluronic acid. Hyaluronic acid makes up the backbone of the specialised extracellular matrix secreted as a part of CC expansion.

Two other main pathways are involved in glucose metabolism in the COC. The pentose phosphate pathway (PPP) results in production of nicotinamide adenine dinucleotide phosphate (NADPH; involved in cytoplasmic integrity and redox state through glutathione) and phosphoribosylpyrophosphate (a substrate for purine synthesis). The polyol pathway involves the oxidation of glucose to produce sorbitol and fructose. The polyol pathway is utilised in hyperglycaemic conditions, for example it is thought to be involved in the diabetic pathology of many tissues (Gabbay, 1975). 


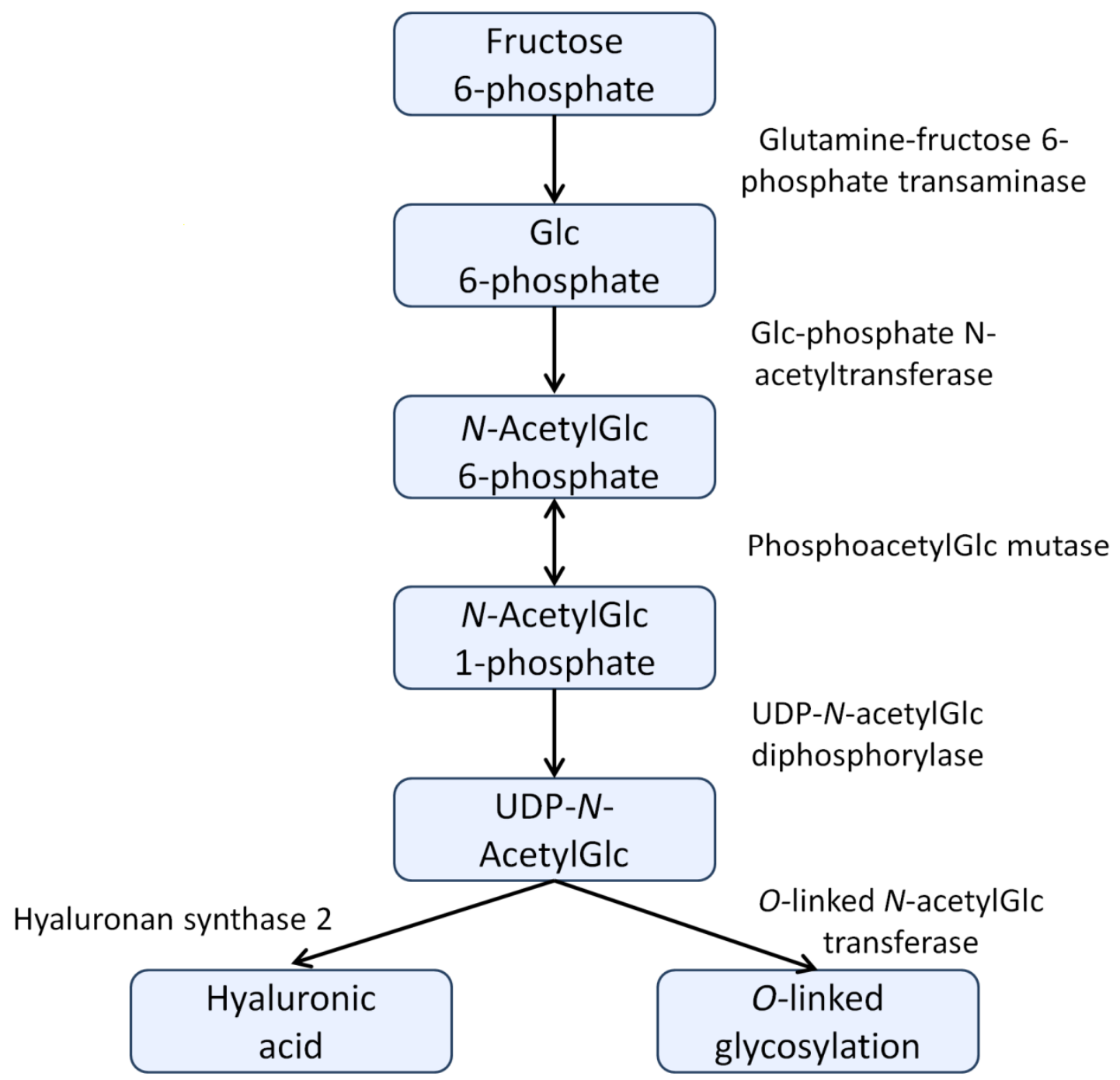

Figure 1-8: The hexosamine biosynthetic pathway. Pathway intermediates/products are contained within the blue boxes. Glc = glucosamine.

\subsubsection{Glucose metabolism in the ovarian follicle}

The mechanism through which glucose uptake by the COC is regulated is species-specific and occurs primarily via facilitative glucose transporters but may also occur via sodium-coupled glucose transporters (Purcell and Moley, 2009; Wang et al., 2012). For example, bovine and ovine GC express mRNA for the facilitative glucose transporter genes Glut1, Glut3, and Glut8 (Nishimoto et al., 2006; Pisani et al., 2008) and mouse and ovine GC additionally express the Glut4 gene (Williams et al., 2001; Roberts et al., 2004). Furthermore, bovine and ovine oocytes express the genes Glut1 and Glut3, with the bovine oocyte additionally expressing the Glut8 gene (Augustin et al., 2001; Pisani et al., 2008). Mouse oocytes express the Glut1, 
and Glut4 genes (Zhou et al., 2000; Roberts et al., 2004). In addition to glucose transporters in mice, oocytes also express monocarboxylate transporters 1,2 , and 4 indicating that murine oocytes are able to take up lactate and pyruvate directly (Hérubel et al., 2002). Oocytes at both the GV and MII stages of meiosis have been reported to consume pyruvate (Harris et al., 2009). In murine oocytes, the concentration of a glucose analogue, 2deoxyglucose, was significantly higher in COC than in DO, indicating that the CC facilitate glucose uptake in oocytes. In vitro studies of bovine $\mathrm{COC}$ revealed that glucose is primarily taken up by the outer CC and gradually moves into the inner CC and oocyte (SuttonMcDowall et al., 2010). In addition, treatment of COC with hCG, which abolishes GJ coupling in a similar manner to $\mathrm{LH}$, reduced the concentration of 2-deoxyglucose in the oocyte to levels similar to those seen in DO (Saito et al., 1994).

It is generally accepted that oocytes metabolise very little glucose compared to CC. Bovine CC consume 23-fold more glucose in comparison to oocytes when assessed per millimetre volume of tissue (Sutton-McDowall et al., 2010). However, both mouse and human oocytes express the enzymes essential for glycolysis (Hardy et al., 1989; Harris et al., 2009) as well as for importing both pyruvate and glucose. Murine oocytes may also metabolise glucose by other pathways as evidenced by expression of hexokinase, glucose-6-phosphate dehydrogenase, GLUTs, and enzymes involved in the PPP (Wang et al., 2012).

The growing oocyte fully depends upon oxidative phosphorylation for ATP production, however has a poor capacity for glucose metabolism (Biggers et al., 1967). The murine oocyte has low PFKP activity, and thus low glycolytic activity. Conversely, murine CC have high activity of enzymes involved in glycolysis such as PFKP and hexokinase (Downs et al., 1996). Thus, the CC produce a significant amount of pyruvate which is able to be transferred to the oocyte (Purcell and Moley, 2009). It is thought that the flux of glucose through the glycolytic pathway in bovine COC remains stable during a 24 hour in vitro maturation (IVM) step despite increased glucose consumption, indicating that glucose may be metabolised by other pathways (Sutton et al., 2003). Increased glycolytic activity in bovine oocytes is associated with increased developmental competence, however this may also lead to increased reactive oxygen species, resulting in oxidative damage (Krisher and Bavister, 1999). Oxidative damage leads to impaired mitochondrial function which, in turn, is associated with decreased developmental competence (Krisher, 2004). 
Activity through the hexosamine biosynthetic pathway is increased towards the end of oocyte maturation in cows due to the requirement for hyaluronic acid (Gutnisky et al., 2007). However, increased activity through this pathway can also lead to increased $O$-linked glycosylation, leading to activation or inactivation of target proteins. This can have negative effects, and has been reported to be a factor in the pathology of diabetes in somatic cells (Brownlee, 2001). Supplementation of IVM media with glucosamine, a substrate which is exclusively metabolised by the hexosamine pathway, inhibited blastocyst development in bovine embryos. This effect was mediated by increased $O$-linked glycosylation (SuttonMcDowall et al., 2006).

Regulation of the PPP pathway is the primary mechanism by which changes in glucose concentration affect nuclear maturation (Downs, 1996). The PPP is more active within the oocyte than the CC, and influences meiotic resumption and developmental competence in both cattle and mice (Downs et al., 1998; Cetica et al., 2002; Wang et al., 2012). Treatment of murine oocytes with a PPP stimulator caused increased numbers of oocyte to undergo GVBD and stimulated intra-oocyte glucose consumption (Wang et al., 2012). Glucose flux through the PPP is increased in response to $\mathrm{FSH}$ in diabetic mice, indicating that this pathway is important for metabolising glucose in hyperglycaemic conditions (Colton et al., 2003). Under normal conditions very little glucose is metabolised via the polyol pathway, with other pathways such as glycolysis or hexosamine biosynthesis being preferred (Brownlee, 2001).

The use of a type I diabetes mouse model has demonstrated the importance of correct glucose levels on oocyte maturation and health (Chang et al., 2005). Pre-ovulatory oocytes from diabetic mice were $30 \%$ smaller than those from control mice. Moreover, oocytes from these mice underwent precocious nuclear maturation. However the proportion of MII oocytes observed was reduced, suggesting a decrease in oocyte competence (Kim et al., 2007). Despite these COC being exposed to hyperglycaemic conditions, the flux through the glycolytic pathway was unaffected as the excess glucose was metabolised through the polyol pathway (Colton et al., 2003; Colton and Downs, 2004). In GC of the type I diabetic mice, GJ communication between the GC and oocyte was decreased by $60 \%$. In addition, Cx37 gene and protein expression was decreased by $36 \%$ (Ratchford et al., 2008). Increased $O$-linked glycosylation and decreased concentrations of FSH are thought to primarily modulate the 
reduction in oocyte competence caused by high glucose concentrations (Hashimoto et al., 2000). Conversely, it is thought that the major pathways involved in reduced oocyte competence as a result of low glucose concentrations are the glycolytic and PPP pathways (Downs et al., 1998).

\subsection{The Influence of Exogenous Factors on Oocyte Quality}

Oocyte quality is likely influenced by a mixture of genetic, endocrine and paracrine signals as well as exogenous factors from the environment. The quality of the ovulated oocyte is particularly important for two reasons. First, the embryonic genome, consisting of paternal and maternal genetic information, is only activated after several cell divisions. Thus, the early development of the embryo in the first few days after fertilisation is largely directed by the oocyte (Kidder and Vanderhyden, 2010). Second, most, if not all, of the oocytes that a female will ever have are contained within the ovary by birth. Thus, oocytes that are ovulated later in life may have been formed 1-1.5 (rodents) or 50 years (humans) earlier (McGee and Hsueh, 2000). Not surprisingly, oocyte quality is reported to decrease over time (Navot et al., 1991).

Poor oocyte quality is a major cause of female infertility. For example, the age-related decline in female fertility is attributed to a concurrent decline in both oocyte quality and numbers (Navot et al., 1991). In humans, infertility is defined as the inability to conceive after one year of unprotected intercourse, with one in six couples experiencing this at some stage of their reproductive life (Anderson, 2010). In 2010, there were $~ 62,000$ assisted reproductive technology (ART) cycles performed in Australia and New Zealand. Of these cycles, $3.5 \%$ used donor oocytes (Macaldowie et al., 2012). The large cost of ART, and frequency of adverse effects, means identification of modifiable factors is important for fertility preservation (Chavarro et al., 2007). A person's lifestyle choices may cause an increase in time to pregnancy, and decrease the chance of a healthy, live birth. However, the current data on the effects of lifestyle on fertility are scarce, contradictory or poorly designed (Anderson, 2010). Thus, further research into the effects of lifestyle on fertility may remove myth-based, unnecessary lifestyle restrictions for couples trying to have a baby (Anderson, 2010). 
Exogenous factors may affect fertility at a number of levels of the reproductive system (Figure 1-9). The coordination of follicular growth is complex, incorporating signals from the pituitary gland (FSH and LH), along with feedback signals from the ovary (oestrogen and progesterone). In addition, feedback from other organs regulates both ovarian and hypothalamic-pituitary function.

Perturbation of the hypothalamic-pituitary-gonadal axis has been observed following exposure to exogenous factors. For example, both acute and chronic exposure of male rats to methylenedioxymethamphetamine (MDMA; ecstasy) leads to reduced mRNA levels of gonadotrophin releasing hormone $(\mathrm{GnRH})$ in the hypothalamus (Dickerson et al., 2008). MDMA is known to target both serotoninergic and dopaminergic pathways, and these also regulate the neurons that produce $\mathrm{GnRH}$. Similarly, $\mathrm{GnRH}$ protein levels are reduced as a result of exposure to $\Delta^{9}$ tetrahydrocannabinol (THC), a compound present in cannabis smoke (Smith et al., 1979). As a result of THC exposure in female rhesus monkeys, inhibition of GnRH signalling causes a reduction in blood gonadotrophin levels (Armstrong, 1986; Park et al., 2004). Additionally, moderate to heavy alcohol consumption in women has been associated with increased plasma oestradiol concentrations (Mendelson et al., 1988). Other known disruptors of the hypothalamic-pituitary-gonadal axis are stress, strenuous exercise, and compounds with oestrogen mimicking properties (Armstrong, 1986).

Exogenous factors may also directly influence the ovary and/or oocytes to reduce fertility. Ovarian follicles, particularly non-growing primordial follicles are sensitive to toxic agents. For example, radiation therapy damages primordial follicles in mice, pigs, monkeys, and humans (Armstrong, 1986). Chemotherapeutic agents also target primordial follicles. Examination of the ovaries of prepubertal girls that had been treated with chemotherapeutic agents found a significantly lower number of oocytes present compared to that in prepubertal girls not exposed to chemotherapy (Himelstein-Braw et al., 1978). Similarly, polycyclic aromatic hydrocarbons, a constituent in automobile exhaust fumes and cigarette smoke, has been shown to destroy primordial follicles in mice (Mattison and Thorgeirsson, 1979). This may also be the case in women, as smokers are reported to undergo menopause significantly earlier than non-smokers (Kaufman et al., 1980). 


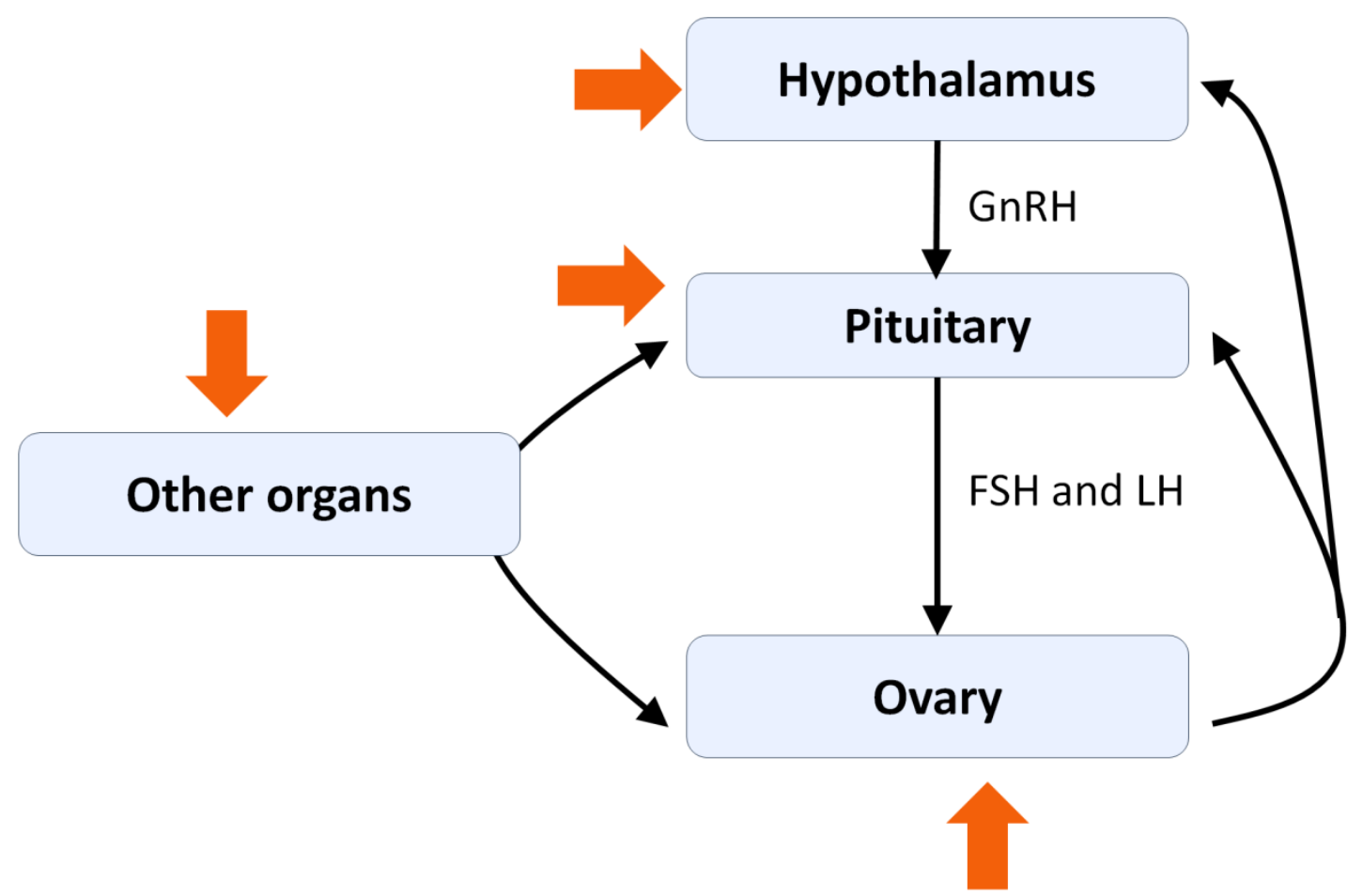

Figure 1-9: Potential sites of action for disruption of female fertility by exogenous factors. As indicated by the orange arrows. Black arrows indicate interactions between the organs.

A number of exogenous factors decrease oocyte quality without causing toxicity. Bisphenol A (BPA), a compound found in plastics, causes spindle abnormalities including distribution of the centriolar material and chromosome alignment in mouse oocytes (Eichenlaub-Ritter et al., 2008). Mouse follicles treated with BPA in vitro had decreased numbers of GC, and a higher proportion of oocytes were unable to resume meiosis (Lenie et al., 2008). BPA exhibits oestrogenic activity, as it binds with both oestrogen receptor $\alpha$ and $\beta$ (Fujimoto et al., 2010). BPA concentrations in the serum of Asian women were correlated with a decrease in MII oocytes (Fujimoto et al., 2010). Caffeine also directly affects the oocyte through its PDE-inhibitory properties. Superovulation in mice was inhibited by treatment with caffeine, with most of the oocytes collected still in the GV stage (Jagiello et al., 1972). Cigarette smoking and compounds found in cigarette smoke have been reported to affect the quality of oocytes in a range of species. Cigarette smoking (either active or passive) causes an increase in the thickness of the zona pellucida in women undergoing in vitro fertilisation (IVF) (Shiloh et al., 2004). Treatment of bovine oocytes with nicotine, the primary addictive agent in cigarettes, inhibited CC expansion, oocyte maturation, and caused meiotic 
abnormalities (Liu et al., 2008). When mice were treated with nicotine, the oocytes displayed premature anaphase, and the proportion of oocytes ovulated was halved compared to controls (Mailhes et al., 2000).

Finally, factors may affect other organs causing an indirect reduction in fertility (Armstrong, 1986). In particular, the liver is a target for such actions as steroid hormone metabolism occurs there. For example, rats that were fed fish that had been exposed to high levels of polychlorinated biphenyls (PCBs) showed increases in hepatic expression of cytochrome P450, an enzyme involved in steroid hormone metabolism. Additionally, the rise in oestradiol levels due to moderate to heavy drinking in women as mentioned above is thought to be due to the breakdown of alcohol in the liver, causing inhibition of oestradiol breakdown (Gill, 2000).

\subsubsection{Lifestyle factors chosen for this study}

Six exogenous lifestyle factors were selected for investigation in this study. These factors were chosen because of their prevalence in our everyday lives (see Table 1-1).

BPA is an endocrine disrupting chemical used in the synthesis of polycarbonate plastics and epoxy resins. BPA accounts for most of the oestrogenic activity that leaches from landfills (Coors et al., 2003) and has been detected in follicular fluid, foetal serum, and amniotic fluid (Ikezuki et al., 2002). Increased BPA concentrations in follicular fluid have been correlated to decreased fertilization rates in both conventional insemination and intracytoplasmic sperm injection (Fujimoto et al., 2010). In Asian women, increased BPA serum concentrations were also linked to reduced oocyte maturation. Urinary BPA concentrations are associated with a reduction in peak plasma oestradiol levels in women during IVF (Mok-Lin et al., 2010), however no relationship was found between urinary BPA and aromatase gene expression levels (Ehrlich et al., 2013). In vitro culture of human oocytes that had been discarded during IVF with BPA resulted in reduced proportions of MII oocytes, and increased proportions of oocytes that had degenerated or spontaneously activated (Machtinger et al., 2013).

During IVF, urinary BPA concentrations are associated with reduced oocyte numbers, reduced fertilisation, and reduced oestradiol concentrations (Ehrlich et al., 2012). 
Caffeine is the most widely used psychoactive compound and is found in many ingested food products including coffee, tea, and chocolate. In a study of women undergoing IVF, $76 \%$ had drunk at least one caffeinated beverage each day in the month prior (Domar et al., 2012). Caffeine has been detected in follicular fluid (Al-Saleh et al., 2010), however the link between caffeine and infertility is contentious (Anderson, 2010). Some studies have found that increased caffeine consumption is associated with a longer time to conception (Hatch and Bracken, 1993; Bolumar et al., 1997) however these failed to adequately take other variables into account, such as smoking. A prospective study showed a significant correlation between not achieving a live birth and consumption of caffeine during IVF (Klonoff-Cohen et al., 2002). A meta-analysis of studies looking at the effect of caffeine and time to conception found that there was no convincing evidence that caffeine increased time to conception (Leviton and Cowan, 2002).

Alcohol (ethanol) is consumed in large amounts throughout most of the world. Approximately $73 \%$ of women undergoing IVF reported drinking at least one serving of alcohol in the month prior (Domar et al., 2012). Mild to moderate consumption of alcohol has a significant negative effect on female fertility (Emanuele et al., 2002). For example, alcoholic women are known to have irregular menstruation or even cessation of menses. Alcohol consumption is correlated with increased plasma oestradiol levels (Van Thiel et al., 1978; Mendelson et al., 1988), and has been associated with anovulation (Mendelson and Mello, 1988). Furthermore, alcohol consumption at levels below those that would cause liver damage can cause temporary anovulation and infertility.

Nicotine is the primary addictive agent found in cigarette smoke. Only $2.5 \%$ of women undergoing IVF reported that they had smoked at least one cigarette in the previous month (Domar et al., 2012). Cigarette smoke is well documented to adversely affect female fertility (Waylen et al., 2009; Anderson, 2010). In vitro or in vivo exposure to nicotine in animal models causes disruption to normal oocyte maturation and ovulation (Liu et al., 2008). Nicotine is metabolised to produce nicotine- $N$-oxide and cotinine. Cotinine has been detected in follicular fluid (Weiss and Eckert, 1989; Zenzes et al., 1996). Nicotine has been reported to inhibit CC expansion and oocyte GVBD, and cause chromosomal abnormalities in bovine COC (Liu et al., 2008). Smokers are 1.6 times more likely to experience infertility than 
non-smokers (Augood et al., 1998), and need approximately twice as many IVF cycles to achieve pregnancy compared with non-smokers (Feichtinger et al., 1997).

The primary psychoactive compound found in cannabis is THC. Cannabis is the most widelyused illicit drug (U.S. Department of Health and Human Services, 2010) and is reported to suppress LH production in women (Mendelson et al., 1986). Acute exposure to THC causes disruption to the menstrual cycle of rhesus monkeys, however tolerance develops during long-term exposure (Smith et al., 1979). In vitro exposure to THC of rat follicles inhibited progesterone, testosterone, and oestradiol production by the follicular cells. In addition, THC stimulated meiotic resumption in rat oocytes (Reich et al., 1982). In a study of couples undergoing IVF, THC was detected in hair samples of 3.7\% of the participants (Pichini et al., 2012) A prospective study showed that women who had smoked marijuana within the past year had 25\% fewer oocytes collected during IVF (Klonoff-Cohen et al., 2006)

MDMA is a recreational drug that is primarily consumed by young people of reproductive age (U.S. Department of Health and Human Services, 2006). Animal studies have found that MDMA suppresses the production of GnRH and testosterone (Dickerson et al., 2008), delays puberty, and affects sperm quality in male rats (Barenys et al., 2010). There are currently no data on the effects of MDMA on IVF outcome, to the best of my knowledge. 
Table 1-1: Measured concentrations of lifestyle factors in biological fluids.

\begin{tabular}{|c|c|c|c|c|c|}
\hline Compound & Species & Concentration & $\begin{array}{l}\text { Biological } \\
\text { Fluid }\end{array}$ & Population details & Reference \\
\hline \multirow[t]{4}{*}{ BPA } & Human & $\begin{array}{c}2.53 \mathrm{ng} / \mathrm{mL} \text { (range } \\
0.0-67.4)\end{array}$ & Serum & $\begin{array}{c}\text { Women undergoing } \\
\text { IVF }\end{array}$ & $\begin{array}{l}\text { (Fujimoto et } \\
\text { al., 2010) }\end{array}$ \\
\hline & Human & $2.08 \pm 0.8 \mathrm{ng} / \mathrm{mL}$ & Serum & Healthy women & $\begin{array}{c}\text { (Ikezuki et al., } \\
\text { 2002) }\end{array}$ \\
\hline & Human & $2.4 \pm 0.8 \mathrm{ng} / \mathrm{mL}$ & $\begin{array}{l}\text { Follicular } \\
\text { fluid }\end{array}$ & $\begin{array}{c}\text { Women undergoing } \\
\text { IVF }\end{array}$ & $\begin{array}{c}\text { (Ikezuki et al., } \\
\text { 2002) }\end{array}$ \\
\hline & Human & $2.2 \pm 1.8 \mathrm{ng} / \mathrm{mL}$ & $\begin{array}{l}\text { Foetal } \\
\text { serum }\end{array}$ & $\begin{array}{l}\text { Umbilical cord blood } \\
\text { from healthy women }\end{array}$ & $\begin{array}{c}\text { (Ikezuki et al., } \\
\text { 2002) }\end{array}$ \\
\hline \multirow[t]{3}{*}{ Caffeine } & Human & $\begin{array}{c}0.913 \mu \mathrm{g} / \mathrm{mL} \\
\text { (range } 0.0-64.04 \text { ) }\end{array}$ & Serum & $\begin{array}{c}\text { Women undergoing } \\
\text { IVF }\end{array}$ & $\begin{array}{c}\text { (Al-Saleh et al., } \\
\text { 2010) }\end{array}$ \\
\hline & Human & $\begin{array}{c}0.701 \mu \mathrm{g} / \mathrm{mL} \\
\text { (range } 0.0-6.48 \text { ) }\end{array}$ & $\begin{array}{l}\text { Follicular } \\
\text { fluid }\end{array}$ & $\begin{array}{c}\text { Women undergoing } \\
\text { IVF }\end{array}$ & $\begin{array}{c}\text { (Al-Saleh et al., } \\
\text { 2010) }\end{array}$ \\
\hline & Human & $10 \mu \mathrm{g} / \mathrm{mL}$ & Plasma & $\begin{array}{l}\text { Males given an oral } \\
\text { dose of } 7 \mathrm{mg} / \mathrm{kg} \\
\text { caffeine }\end{array}$ & $\begin{array}{c}\text { (Axelrod and } \\
\text { Reichenthal, } \\
\text { 1953) }\end{array}$ \\
\hline Ethanol & Human & $800 \mu \mathrm{g} / \mathrm{mL}$ & Blood & $\begin{array}{l}\text { Legal driving limit for } \\
\text { blood alcohol content }\end{array}$ & $\begin{array}{l}\text { New Zealand } \\
\text { Transport } \\
\text { Agency }^{1}\end{array}$ \\
\hline \multirow[t]{3}{*}{ Nicotine } & Human & $30-50 \mathrm{ng} / \mathrm{mL}$ & Plasma & Active smokers & $\begin{array}{c}\text { (Moyer et al., } \\
\text { 2002) }\end{array}$ \\
\hline & Rat & $40-120 \mathrm{ng} / \mathrm{mL}$ & Plasma & $\begin{array}{l}\text { Self-administration } \\
\text { model of nicotine } \\
\text { dependency }\end{array}$ & $\begin{array}{l}\text { (Shoaib and } \\
\text { Stolerman, } \\
\text { 1999) }\end{array}$ \\
\hline & Human & $\begin{array}{c}33 \mathrm{ng} / \mathrm{mL} \text { (range } \\
4-72 \text { ) }\end{array}$ & Plasma & Active smokers & $\begin{array}{c}\text { (Russell et al., } \\
1980 \text { ) }\end{array}$ \\
\hline \multirow[t]{2}{*}{ MDMA } & Human & $\begin{array}{c}0.715 \mu \mathrm{g} / \mathrm{mL} \\
\text { (range } 0.13-2.4 \text { ) }\end{array}$ & Serum & $\begin{array}{l}\text { Patients who } \\
\text { consumed ecstasy at } \\
\text { one nightclub and } \\
\text { were later treated at } \\
\text { two London hospitals }\end{array}$ & $\begin{array}{c}\text { (Greene et al., } \\
\text { 2003) }\end{array}$ \\
\hline & Human & $\begin{array}{c}130.9 \pm 38.6 \\
\mathrm{ng} / \mathrm{mL}\end{array}$ & Plasma & $\begin{array}{c}\text { Male subjects given } 75 \\
\text { mg MDMA }\end{array}$ & $\begin{array}{l}\text { (de la Torre et } \\
\text { al., 2000) }\end{array}$ \\
\hline THC & Human & $67-98 \mathrm{ng} / \mathrm{mL}$ & Plasma & $\begin{array}{l}\text { Peak plasma THC levels } \\
\text { in light and heavy } \\
\text { marijuana users } \\
\text { respectively who had } \\
\text { smoked one marijuana } \\
\text { cigarette }\end{array}$ & $\begin{array}{l}\text { (Lindgren et } \\
\text { al., 1981) }\end{array}$ \\
\hline
\end{tabular}




\subsection{Aims}

The overall aim of this study was to determine whether the health of mammalian oocytes is directly affected by exposure to exogenous lifestyle and environmental factors. To determine the effects of exogenous factors, a rat $\mathrm{COC}$ model was used as the test system. Rats are a commonly used test species for pharmacologically active compounds, enabling investigation into both the in vitro and in vivo effects of lifestyle and environmental factors on COC function. In addition, the large number of antral follicles present in the prepubescent rat ovary facilitated experiments in which large amounts of biological material was required. The presence of a rat breeding colony at VUW meant that female rats were readily available for this study.

A GJ bioassay was optimised to assess the extent of transfer of a fluorescent dye (calcein) from CC to the oocyte following exposure to targeted reagents. In addition, a multiplex quantitative PCR (qPCR) technique was used to measure gene expression levels in individual rat oocytes and CC masses. Subsequently, both the dye transfer assay and multiplex qPCR were tested in combination and validated, to allow for direct comparison of GJ communication and mRNA levels of candidate genes in the same samples. A Western blot protocol was also optimised to detect CX43 and GDF9 proteins in rat COC.

The effect of in vitro exposure to lifestyle factors on rat COC was the major focus of this study utilising the aforementioned assays. The lifestyle factors tested in this study were BPA, caffeine, ethanol, nicotine, MDMA, and THC. Moreover, the effect of in vivo exposure to ecstasy (MDMA) on $\mathrm{COC}$ health was also determined. MDMA was administered to adolescent female rats and thereafter the $\mathrm{COC}$ were extracted, and mRNA and protein expression levels were measured.

The key endpoints of this study were: measurement of GJ communication between the CC and oocyte using an optimised bioassay; qPCR quantification of Cx43, Gfpt1, Pfkp, Atr, Cycs, and Glut1 in CC and Cx37, Gdf9, Bmp15, Atr, Cycs, and Glut1 in oocytes; and quantification of CX43 and GDF9 protein levels using Western blotting. 


\section{GENERAL METHODS}

\subsection{Effect of in vitro exposure to lifestyle factors}

For in vitro studies, ovaries were collected from pre-pubescent Sprague-Dawley female rats as part of routine culling of a breeding colony in the vivarium at the School of Psychology, Victoria University of Wellington (VUW). Euthanasia for tissue collection was included under a general animal ethics approval for rodent research issued by the VUW Animal Ethics Committee (VUW application number 10695).

\subsubsection{Recovery of ovaries}

Prepubescent (21-25 days old) female Sprague-Dawley rats were euthanized by carbon dioxide $\left(\mathrm{CO}_{2}\right)$ asphyxiation. The ovaries were recovered into dissection media (see Appendix 2) supplemented with PDE inhibitors for transportation back to the laboratory. The concentration and type(s) of PDE inhibitors was dependent upon the experiment performed. In the laboratory, the ovaries were transferred into a $35 \mathrm{~mm}$ Petri dish containing dissection media supplemented with PDE inhibitors. Subsequently, the surrounding fatty tissue was trimmed whilst viewing under a Zeiss stereo-zoom dissection microscope (Leica Microsystems; Auckland, NZ).

\subsubsection{Collection of COC for culture}

All of the visible antral follicles $(0.5-1.0 \mathrm{mM}$ in diameter) were punctured using a 20 gauge needle fitted onto a $1 \mathrm{~mL}$ syringe to release the $\mathrm{COC}$ into the dissection media supplemented with PDE inhibitors. The COC that had at least two intact layers of CC were transferred into wells of a non-treated, flat bottomed 48-well polystyrene tissue culture plate (Interlab; Wellington, NZ) and washed twice in $200 \mu \mathrm{L}$ of wash media (see Appendix 2) supplemented with PDE inhibitors. The dimethyl sulfoxide (DMSO) concentration was maintained $1 \%$. Typically 15-20 COC were added to each well.

\subsubsection{GJ Assay}

An acetoxymethyl (AM) ester derivative of the fluorescent dye calcein (Invitrogen; Auckland, NZ) was used to measure GJ transfer from the CC to the oocyte. Calcein fluoresces with excitation and emission wavelengths of 495 and $515 \mathrm{~nm}$, respectively. 
Two distinct GJ assay protocols were used in this study. The first was used to optimise the amount of calcein transfer to the oocyte to allow enhanced assay sensitivity (Figure 2-2). The second protocol was used for all subsequent experiments, including those where the COC were exposed to lifestyle factors (Figure 2-4).

In experiments where the aim was to optimise calcein transfer to the oocyte, COC were exposed to $2 \mu \mathrm{M}$ calcein-AM (see Appendix 2) in $200 \mu \mathrm{L}$ incubation media supplemented with PDE inhibitors, in the presence or absence of treatments, for 10 minutes in incubator conditions of $37{ }^{\circ} \mathrm{C}, 5 \% \mathrm{CO}_{2}$ and $96 \%$ humidity. The $\mathrm{COC}$ were then transferred into $200 \mu \mathrm{L}$ calcein-AM free incubation media with PDE inhibitors, in the presence or absence of treatments, and cultured at $37{ }^{\circ} \mathrm{C}$ either transiently or for up to 24 hours. After being washed in $200 \mu \mathrm{L}$ of incubation media free of PDE inhibitors and treatment, the CC were removed from the oocyte. This was accomplished by vigorous and repeated pipetting of 200 $\mu \mathrm{L}$ of incubation media containing the 15-20 COC. The DO were photographed to allow future analysis of the presence or absence of a GV (Figure 2-1). Oocytes were either categorised as containing (see Figure 2-3A) or not containing a visible GV (see Figure 2-3B). In cases where the GV was not clearly identifiable, the oocyte was categorised as not containing a GV for analysis purposes. The degree of fluorescence emitted by the DO was measured using a Leica inverted microscope (CTR4000) with a CRAIC fluorescence detection system (model QDI302; Leica Microsystems; Wetzlar, Germany). The settings for these measurements were 50 milliseconds over the wavelength range of 300 to $700 \mathrm{~nm}$. A dark scan was performed to determine the amount of electronic noise when the excitation light is turned off. Fluorescence was measured near the centre of each oocyte. 
A

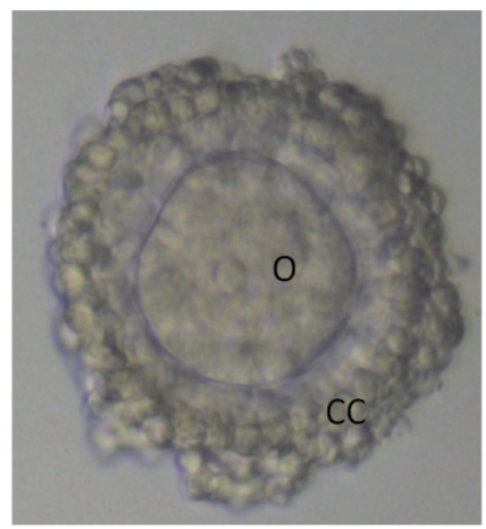

C

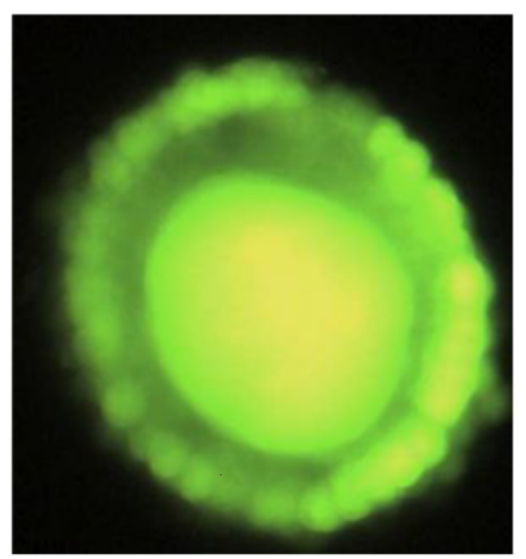

B

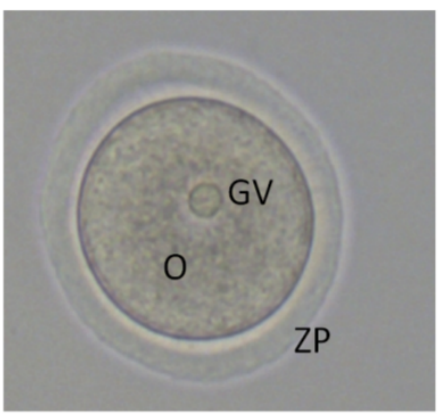

D

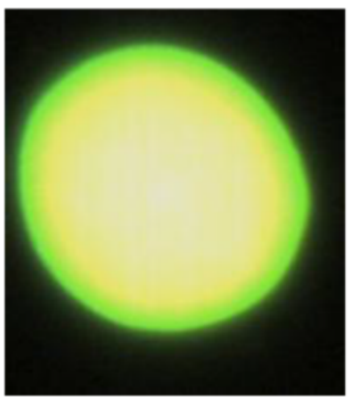

Figure 2-1: Photographs depicting rat $\operatorname{COC}(A, C)$ and denuded oocytes $(B, D)$ before and after exposure to calcein-AM. Indicated on the photographs are the cumulus cells (CC), oocyte (O), germinal vesicle (GV), and zona pellucida (ZP).

\begin{tabular}{|c|c|c|}
\hline $\begin{array}{c}\text { Calcein-AM } \\
\text { exposure } \\
\text { ( } \pm \text { treatment) }\end{array}$ & $\begin{array}{c}\text { Incubation } \\
\text { ( } \pm \text { treatment) }\end{array}$ & $\begin{array}{c}\text { Denude oocyte, } \\
\text { measure } \\
\text { fluorescence }\end{array}$ \\
\hline $\begin{array}{c}10 \\
\text { minutes }\end{array}$ & $1-24$ hours & \\
\hline
\end{tabular}

Figure 2-2: Schematic overview of the protocol used to optimise dye transfer in the GJ assay. 
A

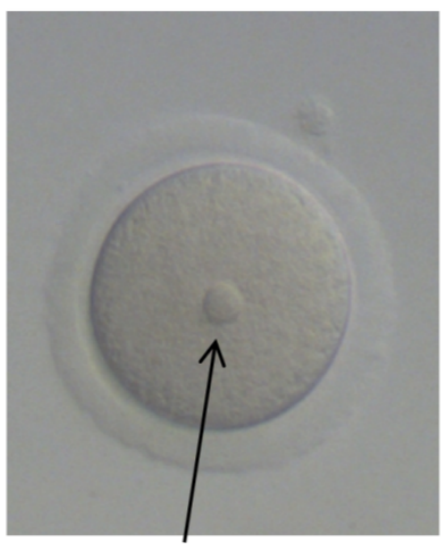

Germinal vesicle
B

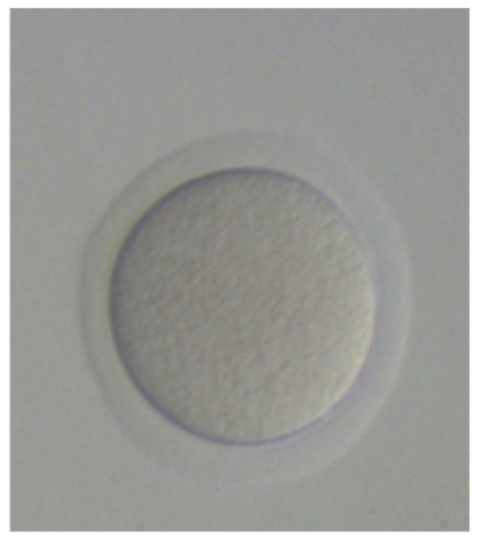

Figure 2-3: Photographs depicting an oocyte with a visible germinal vesicle (A) and without a germinal vesicle (B).

\begin{tabular}{cccc}
$\begin{array}{c}\text { Incubation } \\
( \pm \text { treatment })\end{array}$ & $\begin{array}{c}\text { Calcein-AM } \\
\text { exposure } \\
( \pm \text { treatment })\end{array}$ & $\begin{array}{c}\text { Post-calcein } \\
\text { Incubation } \\
( \pm \text { treatment })\end{array}$ & $\begin{array}{c}\text { Denude oocyte, } \\
\text { measure } \\
\text { fluorescence }\end{array}$ \\
\hline $1-48$ hours & $\begin{array}{c}10 \\
\text { minutes }\end{array}$ & 1 hour \\
\hline
\end{tabular}

Figure 2-4: Schematic overview of the optimised GJ assay protocol.

For all other experiments, $\mathrm{COC}$ were cultured at $37^{\circ} \mathrm{C}$ in $200 \mu \mathrm{L}$ incubation media with PDE inhibitors in the presence or absence of treatment for up to 48 hours. Following the incubation period, the COC were exposed to $2 \mu \mathrm{M}$ calcein-AM for 10 minutes at $37^{\circ} \mathrm{C}$. The COC were then transferred into calcein-AM free media with PDE inhibitors and with or without treatment and incubated at $37^{\circ} \mathrm{C}$ for a further 1 hour to allow dye transfer. In those experiments where the COC were not collected for RNA extraction, the COC were washed twice in $200 \mu \mathrm{L}$ incubation media free of PDE inhibitors and treatment and the 15-20 oocytes within each well were denuded from their CC as described above. The DO were photographed for GV analyses and the fluorescence was measured using the Leica inverted microscope as described previously. 
In those experiments where the COC were collected for RNA extraction, individual COC were transferred into wells of a 96-well plate (Thermo Fisher Scientific; Auckland, NZ) where they were washed twice in $100 \mu \mathrm{L}$ phosphate buffered saline (PBS). The COC were transferred into $10 \mu \mathrm{L}$ PBS and photographed. Thereafter, the oocytes were separated from the CC and the DO were transferred into $100 \mu \mathrm{L}$ incubation media free of PDE inhibitors and treatment. The DO were photographed and fluorescence was measured within the oocyte as described previously. Thereafter the DO were washed twice more in $100 \mu \mathrm{L}$ PBS before being transferred into $10 \mu \mathrm{L}$ fresh PBS. The 96-well plates containing the separated CC masses and DO each in $10 \mu \mathrm{L}$ PBS were stored at $-80^{\circ} \mathrm{C}$.

\subsubsection{GJ assay data analysis}

Each oocyte was considered to be a discrete variable, so that the number of samples was considered to be the total number of readings across all replicates. For each replicate experiment, the geometric mean value was calculated for the raw fluorescence measurements of the control group (usually the untreated group at 1 hour). Relative fluorescence values were then calculated by dividing the raw fluorescence measurement for each individual oocyte by the geometric mean of the control group. The geometric mean and $95 \%$ confidence interval of the relative fluorescence values were calculated for each experimental group. All statistical analyses of the data were performed using the GraphPad Prism 5 statistics program.

Experiments were analysed using the appropriate statistical test, namely either the MannWhitney test (one-way analysis), the Kruskal-Wallis test with Dunn's multiple comparison post-hoc test (one-way analysis), or the two-way analysis of variance (ANOVA; two-way analysis). Where a two-way ANOVA was utilised, the data were first tested for normality using the D'Agostino \& Pearson normality test. Data that was not normally-distributed was transformed using an appropriate transformation and tested for normality again. If no transformation method yielded normally-distributed data, the data points were ranked and a two-way ANOVA was performed on the ranks.

\subsubsection{COC culture for Western blotting}

For the Western blotting experiments, the dissection, wash and incubation media contained the PDE inhibitors, dipyridamole $(50 \mu \mathrm{M})$ and rolipram $(50 \mu \mathrm{M})$. Following extraction, COC 
were transferred into a 48-well plate and washed twice in $200 \mu \mathrm{L}$ wash media. Subsequently, the COC were transferred into $200 \mu \mathrm{L}$ incubation media containing PDE inhibitors in the absence or presence of treatment. The $\mathrm{COC}$ were then incubated at $37{ }^{\circ} \mathrm{C}$ with $5 \% \mathrm{CO}_{2}$ and $96 \%$ humidity for up to 25 hours.

After the culture period, the COC were washed twice in $200 \mu \mathrm{L}$ PBS. A known number of COC (100 COC/group) were transferred into a microcentrifuge tube, and the total volume was made up to $500 \mu \mathrm{L}$ with PBS. The microcentrifuge tubes were centrifuged at $800 \times g$ for 10 min, and the supernatant was removed and dispensed into a culture dish. The supernatant was checked under the dissection microscope to ascertain whether any $\mathrm{COC}$ were accidentally removed from the pellet. The COC pellet was then resuspended in PBS supplemented with protease inhibitors and stored at $-20^{\circ} \mathrm{C}$.

Due to the time taken to extract the COC, only 100 COC per treatment group were collected in each experiment and these were later pooled if necessary.

\subsubsection{CC serial dilution}

Rat ovaries were collected in PBS, and the COC were extracted into fresh PBS as described previously. The CC were stripped from the oocyte by vigorous and repeated pipetting and the oocytes were removed from the resulting cell suspension and discarded. A $50 \mu \mathrm{L}$ aliquot of the CC suspension was mixed with $5 \mu \mathrm{L} 0.4 \%$ Trypan blue stain, and the cells were counted using a haemocytometer under an inverted microscope at $400 x$ magnification. The total cell count was determined and the CC were serially diluted in triplicate with PBS to produce samples containing between 280 and $18,000 \mathrm{CC}$. The samples were stored at $-80^{\circ} \mathrm{C}$ prior to RNA extraction.

\subsection{Effect of in vivo exposure to methylenedioxymethamphetamine (MDMA)}

\subsubsection{Treatment regimen}

All experiments involving administering MDMA to rats were approved by the VUW Animal Ethics Committee (VUW application number 2011R16).

Pre-pubescent (21-25 day old) female Sprague-Dawley rats were weaned and housed in groups of 4 in a temperature controlled room at $22{ }^{\circ} \mathrm{C}$ and $55 \%$ humidity, with a 12 hour 
light/dark cycle. Food and water was available ad libitum. At the beginning of each experimental week, MDMA (kindly donated by Mr Richard Moore and Prof Susan Schenk, School of Psychology, VUW), was diluted in sterile saline to a concentration of $2.5 \mathrm{mg} / \mathrm{mL}$ and stored at $4{ }^{\circ} \mathrm{C}$. The rats were administered either the vehicle (sterile saline; $\mathrm{N}=12$ ) or 5 $\mathrm{mg} / \mathrm{kg} / \mathrm{MDMA}(\mathrm{N}=12)$ via intraperitoneal (ip) injection. Each rat was administered one injection every 24 hours for 3 days (total of 3 injections). Due to logistics, three replicate experiments were undertaken (i.e. 4 rats/group/replicate experiment) over three consecutive weeks.

To check the efficacy of the MDMA, locomotor activity of all rats following each injection was measured using Activity Monitor Version 5 software (Med Associates Inc.; St Albans, VT, USA). This test was performed by placing each rat in a locomotor activity box $(40 \times 40 \mathrm{~cm})$ containing 16 infrared beams and corresponding sensors. When the subject stops a beam from reaching the sensor, this event is recorded. As a subject moves, a 'box' is established which is 3 infrared beams in width and length. The subject is considered ambulatory when it breaks more than 3 beams outside the 'box' within 1000 milliseconds. If the subject fails to meet these criteria, it is considered to be in a resting phase until such time as it meets the ambulatory criteria. The software measures ambulatory counts, which are defined as the total number of beams broken while the subject is in an ambulatory episode.

The rats were habitualised in the locomotor activity boxes for 30 minutes, with the Activity Monitor software running to measure their activity. The activity boxes were located inside cupboards to create a darkened environment, and a white noise generator was used throughout the measurement of locomotor activity to minimise environmental disturbances. After habitualisation, the rats were administered their daily injection of either $5 \mathrm{mg} / \mathrm{kg}$ MDMA or saline ( $N=4 /$ treatment group/week) via ip injection. The rats were returned to the locomotor activity boxes where their activity was monitored for a further 60 minutes. The rats were then returned to the temperature-controlled room. Measurement of locomotor activity was repeated following every injection.

Approximately 18 hours after the final injection, the rats were sacrificed by $\mathrm{CO}_{2}$ asphyxiation. The ovaries were collected into sterile PBS. 


\subsubsection{Collection of COC}

The ovaries from control and MDMA-treated rats were kept separate at all times. The ovaries were transferred into a $35 \mathrm{~mm}$ Petri dish containing sterile PBS and the surrounding fatty tissue was trimmed. The follicles were punctured using a 20 gauge needle, releasing the $\mathrm{COC}$ into the PBS. The $\mathrm{COC}$ were then transferred into a fresh dish containing sterile PBS for either gene or protein expression studies.

The COC that were collected for gene expression were transferred singly into $10 \mu \mathrm{L}$ of PBS in a 96-well plate and thereafter DO and CC were isolated separately as previously described. The DO were photographed before being transferred into $10 \mu \mathrm{L}$ PBS. The 96-well plates containing the isolated DO and $\mathrm{CC}$ were then stored at $-80{ }^{\circ} \mathrm{C}$ until gene expression studies were performed.

For protein analysis, $200 \mathrm{COC} /$ group were transferred into a microcentrifuge tube. Additional PBS was added to make the volume up to $500 \mu \mathrm{L}$. The microcentrifuge tubes were centrifuged at $800 \times \mathrm{g}$ for $10 \mathrm{~min}$. The supernatant was transferred into a culture dish to check for the accidental removal of any COC. The COC pellet was then resuspended in $15 \mu \mathrm{L}$ PBS containing protease inhibitors. The samples were stored at $-20^{\circ} \mathrm{C}$ until required.

\subsection{Quantitative PCR (qPCR)}

For the optimisation steps for each qPCR gene set, cDNA from pools of $50 \mathrm{COC}$ were used as the template. All subsequent qPCR experiments were performed on individual DO and associated CC masses.

\subsubsection{RNA extraction and cDNA synthesis}

Total RNA was extracted using the ArrayPure Nanoscale RNA purification kit (Epicentre Biotechnologies; Madison, WI, USA) following the manufacturer's instructions. In detail, 30 $\mu \mathrm{L}$ of Nanoscale Lysis solution containing $5 \mu \mathrm{g}$ of Proteinase $\mathrm{K}$ was added to each sample, and mixed thoroughly by pipetting. If the sample was in a 96-well plate, this solution was transferred to a microcentrifuge tube before proceeding. The contents of the microcentrifuge tubes were vortexed for 1 minute before being incubated at $65{ }^{\circ} \mathrm{C}$ for 15 minutes. The samples were then placed on ice for 5 minutes, followed by the addition of 18 $\mu \mathrm{L}$ of MPC Protein Precipitation Reagent and mixing by vortex for 10 seconds. The precipitated protein was pelleted by centrifugation at $12,000 \times g$ at $4{ }^{\circ} \mathrm{C}$ for 7 minutes, and 
the supernatant was transferred into a fresh microcentrifuge tube. In order to precipitate the RNA, $50 \mu \mathrm{L}$ of isopropanol was added to the supernatant and vortexed for 30 seconds before being pelleted by centrifugation at $12,000 \times g$ at $4{ }^{\circ} \mathrm{C}$ for 5 minutes. The isopropanol was discarded, and the RNA pellet was air dried for 5 minutes. The pellet was resuspended in $20 \mu \mathrm{L}$ of DNase buffer containing $0.5 \mathrm{U}$ DNase I enzyme by vortexing for 1 minute. To remove any contaminating genomic DNA, this solution was incubated at $37{ }^{\circ} \mathrm{C}$ for 30 minutes. Following the incubation, $20 \mu \mathrm{L}$ of $2 x$ Nanoscale Lysis solution was added and the tubes were vortexed for five seconds. Subsequently $20 \mu \mathrm{L}$ of MPC protein precipitation reagent was added, and the tubes were vortexed for a further 10 seconds. To precipitate any remaining protein, samples were then placed on ice for 5 minutes before being centrifuged at $12,000 \times g$ at $4{ }^{\circ} \mathrm{C}$ for 5 minutes. The supernatant was transferred to a fresh microcentrifuge tube and RNA was precipitated with the addition of $50 \mu \mathrm{L}$ of isopropanol. The tubes were vortexed for 30 seconds and then centrifuged at $12,000 \times g$ at $4{ }^{\circ} \mathrm{C}$ for 5 minutes. The isopropanol was discarded, and the RNA pellet was washed with $50 \mu \mathrm{L}$ of $70 \%$ ethanol. The samples were centrifuged at $12,000 \times g$ at $4{ }^{\circ} \mathrm{C}$ for a further 3 minutes and the ethanol was discarded. The RNA pellet was allowed to air dry for 5 minutes before being resuspended in $10 \mu \mathrm{L}$ Ultrapure distilled $\mathrm{H}_{2} \mathrm{O}$ (Invitrogen) by vortexing for 2 minutes. To ensure full resuspension of the RNA pellet, the samples were incubated at $55{ }^{\circ} \mathrm{C}$ for 2 minutes before being either stored at $-80{ }^{\circ} \mathrm{C}$ until required or added directly to the complementary DNA (cDNA) synthesis reaction as described below.

The synthesis of cDNA from total RNA was performed using the SuperScript ${ }^{\circledR}$ Vilo $^{\text {TM }}$ cDNA Synthesis Kit (Invitrogen) following manufacturer's instructions. In detail, the entire total RNA sample $(10 \mu \mathrm{L})$ was added to $4 \mu \mathrm{L}$ of $5 \mathrm{x}$ VILO reaction mix, $2 \mu \mathrm{L}$ of $10 \mathrm{x}$ SuperScript ${ }^{\circledR}$ Vilo ${ }^{\mathrm{TM}}$ enzyme and made up to a total volume of $20 \mu \mathrm{L}$ with distilled $\mathrm{H}_{2} \mathrm{O}\left(\mathrm{dH}_{2} \mathrm{O} ; 4 \mu \mathrm{L}\right)$. Following mixing by vortex, the samples were incubated in the Corbett Rotor-Gene 6000 machine (model no. 11754-050; Corbett Life Science; Sydney, Australia) under the following conditions: $25{ }^{\circ} \mathrm{C}$ for 10 minutes, $42{ }^{\circ} \mathrm{C}$ for 120 minutes, $85{ }^{\circ} \mathrm{C}$ for 5 minutes. The samples were removed immediately following cDNA synthesis and stored at $-20^{\circ} \mathrm{C}$ until required.

\subsubsection{Multiplex $q P C R$}

Gene expression was quantified using multiplex TaqMan qPCR. TaqMan probes contain a fluorophore (FAM, HEX, ROX, or CY5) at the $3^{\prime}$ end that differ in excitation and emission 
wavelengths, an oligonucleotide sequence specific to the query gene, and a quencher at the 5 ' end. The fluorophore does not emit fluorescence due to the close proximity of the quencher. During the annealing steps of the amplification cycle, the primers and TaqMan probe bind to the template sequence. As the DNA polymerase moves along the sequence during the extension step, it displaces the fluorophore from the probe containing the quencher, allowing the emission of fluorescence.

Each reaction contained optimised concentrations of the appropriate forward and reverse primers (Invitrogen) and TaqMan probes (Sigma; Auckland, NZ) a pre-made master mix (Agilent Technologies; Santa Clara, USA), cDNA template, and was made up to a total volume of $52 \mu \mathrm{L}$ with UltraPure $\mathrm{dH}_{2} \mathrm{O}$. This reaction was mixed by vortex before being pipetted in duplicate $25 \mu \mathrm{L}$ aliquots into $0.1 \mathrm{~mL}$ strip tubes. Each reaction run contained a calibrator control and a no template control. The calibrator control contained pooled cDNA that had been isolated from a pool of rat $\mathrm{COC}$, and was included in every reaction to enable normalisation against differences in reaction.

The Brilliant II SYBR ${ }^{\circledR}$ Green QPCR Master Mix (Agilent Technologies) was used to optimise the primer concentrations. The Brilliant ${ }^{\circledR}$ Multiplex QPCR Master Mix (Agilent Technologies) was used for all other experiments.

The samples were placed in the Corbett Rotor-Gene 6000 machine for thermal cycling and measurement of fluorescence. The thermal cycle conditions were as follows: 10 minutes at $95{ }^{\circ} \mathrm{C}$, then 40 repeats of 15 seconds at $95{ }^{\circ} \mathrm{C}$ and 60 seconds at $60{ }^{\circ} \mathrm{C}$. To check primer specificity, a final melt step was added when SYBR green technology was used, involving heating the samples from $60{ }^{\circ} \mathrm{C}$ to $95{ }^{\circ} \mathrm{C}$, with 5 seconds incubation for each $0.5^{\circ} \mathrm{C}$ rise in temperature. The qPCR results were analysed using the Corbett Rotor-Gene 600 Series Software 1.7.

\subsubsection{Primers and probes}

To determine gene expression levels, quadriplex reactions containing three query genes and the reference gene 60S ribosomal protein L19 (Rp/19) were prepared and measured using qPCR. Three different quadriplex reactions were included in this study. The first quadriplex reaction contained the oocyte-specific query genes growth differentiation factor 9 (Gdf9), bone morphogenetic protein 15 (Bmp15), and connexin 37 (Cx37). The second multiplex 
reaction contained the query genes connexin $43\left(C_{x} 43\right)$, glutamine-fructose-6-phosphate aminotransferase (Gfpt1), and phosphofructokinase, platelet (Pfkp). The third multiplex reaction contained the query genes ataxia telangiectasia and Rad3 related (Atr), cytochrome c (Cycs), and glucose transporter 1 (Glut1).

For each query gene, a forward oligonucleotide primer, reverse oligonucleotide primer, and TaqMan probe were designed using the Beacon Designer software package (Premier Biosoft International, USA). The same Rp/19 forward and reverse primers and TaqMan probe were used in each multiplex reaction. The sequences of each forward and reverse primer set and each TaqMan probe are listed in Table 2-1 and Table 2-2, respectively.

Table 2-1: Nucleotide sequences for forward and reverse primers for qPCR, including NCBI accession numbers, for each gene investigated.

\begin{tabular}{|c|c|c|}
\hline Gene & NCBI Accession No & Forward $(F)$ and reverse $(R)$ primers \\
\hline \multirow{2}{*}{ Gdf9 } & \multirow{2}{*}{ NM_021672 } & F- GGAACACTTACTCAAATCGGTCTTG \\
\hline & & R- GCCTTGCTGGAAGACATGGG \\
\hline \multirow{2}{*}{ Bmp15 } & \multirow{2}{*}{ NM_021670 } & F- AACGAGGTAGCATACCAACTAATCAG \\
\hline & & R- GTTCGACATTTAGGGACCCAAGG \\
\hline \multirow{2}{*}{$C \times 37$} & \multirow{2}{*}{ NM_021654 } & F- GACGGTCGTCTTCGCATTCG \\
\hline & & R- ACGCCACTGGCCATAGAGG \\
\hline \multirow{2}{*}{$C \times 43$} & \multirow{2}{*}{ NM_012567 } & F- CAGCCATTAGCCATCGTG \\
\hline & & R- CGGTTTAAATCTCCAGGTCATC \\
\hline \multirow{2}{*}{ Gfpt1 } & \multirow{2}{*}{ NM_001005879 } & F- AGCAGCCAGAATCTGTTG \\
\hline & & R- GTGGTAACTTGTGCCACA \\
\hline \multirow{2}{*}{$P f k p$} & \multirow{2}{*}{ NM_206847 } & F- GGGCATAACCAACCTGTG \\
\hline & & R- CGATCTCACCATTCTTAGCC \\
\hline \multirow{2}{*}{ Atr } & \multirow{2}{*}{ XM_003754445 } & F- GGTAGAATCTCTCCACTCCAGTCA \\
\hline & & R- AAGCCATTTCTCTGTGATGAGCAA \\
\hline \multirow{2}{*}{ Cycs } & \multirow{2}{*}{ NM_012839 } & F- CCCGTGCTTCAGTGTGTGTAAA \\
\hline & & R- CAGTGGTGAAAGGCAGCATCA \\
\hline \multirow{2}{*}{ Glut1 } & \multirow{2}{*}{ NM_138827 } & F- CCTTGCCTGAGACCAGTTGAA \\
\hline & & R- ACAGCAGGGCAGGAGTGTC \\
\hline \multirow{2}{*}{ Rp/19 } & \multirow{2}{*}{ NM_031103 } & F- CCCAATGAAACCAACGAAA \\
\hline & & R- GGGAATGGACAGTCACAG \\
\hline
\end{tabular}


Table 2-2: Nucleotide sequences for TaqMan probe sequences for qPCR for each gene investigated.

\begin{tabular}{cl}
\hline Gene & Probe Sequence \\
\hline Gdf9 & [6-FAM]CACTCTGAACAACTCCGCTGCTTCCTCCT[BHQ1] \\
Bmp15 & [HEX]CACTGTGGTCTACCGCCATCAACTTCATCTAGT[BHQ1] \\
CX37 & [ROX]CCTCCAGCACACTCTTACACAGCACACTC[BHQ2] \\
CX43 & [6-FAM]CTTCCAGCAGAGCCAGCAGC[BHQ1] \\
Gfpt1 & [HEX]CAGAATCAACCGCCACACCG[BHQ1] \\
Pfkp & [ROX]TCCAGAAGACCGCTCCACTCC[BHQ2] \\
Atr & [6-FAM]ACTCCTTGCCAGACTGCTGAGATGCGAA[BHQ1] \\
Cycs & [ROX]TGCTAAACACCAGGACGGAACTATTCTTGACA[BHQ2] \\
Glut1 & [HEX]TGTCTTCAGCAGTAAGTTCTCAGCCTCCGA[BHQ1] \\
Rpl19 & [Cy5]CCAATGCCAACTCTCGTCAACAGATCAGGAAG[BHQ3]
\end{tabular}

Table 2-3: Optimal final concentrations (nM) of forward and reverse primers and TaqMan probes in qPCR reactions.

\begin{tabular}{cccc}
\hline Gene & Forward Primer & Reverse Primer & Probe \\
\hline Gdf9 & 200 & 100 & 100 \\
Bmp15 & 300 & 200 & 100 \\
Cx37 & 300 & 100 & 50 \\
Cx43 & 50 & 300 & 50 \\
Gfpt1 & 100 & 200 & 100 \\
Pfkp & 300 & 300 & 100 \\
Atr & 200 & 200 & 50 \\
Cycs & 100 & 100 & 100 \\
Glut1 & 100 & 200 & 100 \\
Rpl19 & 300 & 200 & 100 \\
\hline
\end{tabular}

\subsubsection{Multiplex optimisation}

The optimal concentration of the forward and reverse primers for each gene was determined by titration of the primer concentrations between $50 \mathrm{nM}$ and $300 \mathrm{nM}$. This was performed for each gene individually using SYBR green technology. The combination of primer concentrations that gave the lowest cycle threshold $\left(C_{T}\right)$ value without compromising on endpoint fluorescence was selected. The optimal probe concentration was determined for each gene individually by titration of the probe concentration between $50 \mathrm{nM}$ and 300 
nM. As each probe was labelled with a fluorophore, the TaqMan technology was used. The optimal primer and probe concentrations for each gene are shown in Table 2-3.

The reaction efficiency of each gene in each quadriplex reaction was determined after the optimised conditions were determined for each gene. A cDNA dilution series was prepared, with each successive dilution containing half the concentration of cDNA. If the reaction had $100 \%$ efficiency, the $C_{T}$ value for each successive dilution would differ by 1.0 , as $C_{T}$ values are on a logarithmic scale. The reaction efficiencies for each of the genes are shown in Table $2-4$.

Table 2-4: The amplification efficiency for each gene in three different qPCR quadriplex reaction sets.

\begin{tabular}{ccc}
\hline Gene & Threshold & Efficiency (\%) \\
\hline Quadriplex 1 & 0.03 (slope correct) & 92 \\
Bmp15 & & 93 \\
Gdf9 & 98 \\
CX37 & 92 \\
Rpl19 & 0.03 (slope correct) & \\
Quadriplex 2 & & 90 \\
Cx43 & & 91 \\
Gfpt1 & 95 \\
Pfkp & 98 \\
Rpl19 & & 101 \\
Quadriplex 3 & 96 \\
Atr & 0.07 & 98 \\
Cycs & 99 \\
Glut1 & \\
Rpl19 & & \\
\hline
\end{tabular}




\subsection{5 qPCR Analysis}

Each oocyte was considered to be a discrete variable, so that the number of samples was considered to be the total number of readings across all replicates. Relative gene expression, compared to the control group, was calculated as described previously (Livak and Schmittgen, 2001). The CC samples were further corrected for the reference gene Rp/19, to give gene expression relative to RNA input. The oocyte samples were corrected for interassay reaction differences (i.e. internal calibrator control), to give total gene expression per single oocyte.

For each CC sample, the $C_{T}$ value for $R p / 19$ was subtracted from the $C_{T}$ value of the query gene, to give the $\Delta C_{T}$ value. For the oocyte samples, the $C_{T}$ value of the calibrator control for each query gene was subtracted from the $C_{T}$ value for each oocyte sample for the same query gene. The average $\Delta C_{T}$ of the control group was calculated, and this was subtracted from the $\Delta C_{T}$ values for each sample to give the $\Delta \Delta C_{\mathrm{T}}$. The $\Delta \Delta \mathrm{C}_{\mathrm{T}}$ values were converted to linear values by applying the formula $2^{-(\Delta \Delta C)}{ }_{T}$. This formula gives a positive change in linear values as mRNA levels increase. Any sample that had a $C_{T}$ value for $R p / 19$ of $\geq 30$ was excluded from analysis due to insufficient template. Samples that had a $C_{T}$ value for the query genes that was out of the detection range (>34) were given a $C_{T}$ value of 40 for analysis purposes.

In order to plot the data, the formula $2^{-\left(\text {Average } \Delta \Delta C_{T}\right)}$ was applied to the average $\Delta \Delta C_{T}$ for each group, to give the mean relative change in gene expression. The upper and lower errors were calculated using the formula $2^{-\left(A v e r a g e \Delta \Delta C_{T} \pm \text { SEM) }\right.}$, where SEM is the standard error of the mean.

Statistical analysis was performed using the GraphPad Prism 5 software program. In general, either a Kruskal-Wallis test or a two-way ANOVA was employed to analyse the data. Where a two-way ANOVA was used, the data were first tested for a normal distribution using the D'Agostino \& Pearson normality test. Where the data were not normally-distributed, the appropriate transformation method was used and the data were tested for a normal distribution again. If no transformation method made the data normally-distributed, the data were ranked and a two-way ANOVA was performed on the ranked data. 


\subsection{Western Blotting}

\subsubsection{Protein quantification}

For Western blots, the total amount of protein loaded was determined using a bicinchoninic (BCA) based assay (Thermo Scientific; Auckland, NZ). This assay relies on the reduction of $\mathrm{Cu}^{+2}$ ions to $\mathrm{Cu}^{+1}$ by protein in an alkaline environment. Each $\mathrm{Cu}^{+1}$ ion is chelated by two $\mathrm{BCA}$ molecules, and this complex has strong absorbance at $592 \mathrm{~nm}$.

The microplate procedure was used as described below. Bovine serum albumin was dissolved in PBS at concentrations of $0,5,10,20,30,40,50,75$, and $100 \mu \mathrm{g} / \mathrm{mL}$. An aliquot ( 67 COC; usually in $10 \mu \mathrm{L}$ ) of the crude COC lysate collected for Western blotting was diluted with $80 \mu \mathrm{L}$ PBS buffer. Each COC sample was serially diluted to produce two sample dilutions, each containing half the amount of protein as the previous sample solution. The assay was performed in a 96-well plate. The assay plate was prepared by dispensing $25 \mu \mathrm{L}$ of each standard and sample solution into the appropriate wells. The standards were assayed in triplicate and the samples were assayed in duplicate as described below.

The BCA working reagent was prepared by mixing 50 parts of BCA Reagent $A$ with 1 part of BCA Reagent B. This working reagent $(200 \mu \mathrm{L})$ was added to each well, and the plate was mixed for 30 seconds using an Eppendorf MixMate plate shaker (Eppendorf; Hamburg, Germany). The plate was sealed and incubated at $37{ }^{\circ} \mathrm{C}$ (standard protocol) or $60{ }^{\circ} \mathrm{C}$ (optimised protocol) for 2 hours. The plate was removed from the incubator and allowed to cool to room temperature. The absorbance of the wells at $490 \mathrm{~nm}$ was measured using a VICTOR3 multilabel plate reader (Perkin Elmer, Waltham, MA, USA).

The data were analysed by subtracting the absorbance of the blank from the standard and sample absorbance values. A standard curve was created using a best-fit linear regression of the absorbance values of the standards from which the protein concentrations of the samples were calculated. The amount of protein in each of the serial dilutions was corrected for the dilution factor, and an average protein concentration over the two serial dilutions was calculated. 


\subsubsection{SDS-PAGE}

Proteins were separated by molecular weight by sodium dodecyl sulphate polyacrylamide gel electrophoresis (SDS-PAGE). Polyacrylamide gels, with a $13.5 \%$ separating gel and $4 \%$ stacking gel (Appendix 2), were prepared one day in advance and stored overnight at $4{ }^{\circ} \mathrm{C}$ in paper towels soaked in $\mathrm{dH}_{2} \mathrm{O}$.

Sample buffer was added to each crude COC lysate to a final concentration of $2 x$ (Appendix 2). Each blot also contained a pre-stained SDS-PAGE Low-Range standard (BioRad; Auckland, $\mathrm{NZ}$ ). All the samples were denatured at $95^{\circ} \mathrm{C}$ for 10 minutes before being briefly centrifuged. An SDS-PAGE running cassette was set up using the polyacrylamide gel and 1x SDS-PAGE running buffer (Appendix 2). The entire crude COC lysate (minus the aliquot utilised for the BCA assay) was added into the appropriate well. Therefore, each COC sample lane contained protein from $\sim 133 \mathrm{COC}$ and this was kept constant between treatment groups and experimental replicates. If the entire volume of sample would not fit in one well, the gel was electrophoresed at 150 volts for 5 minutes to allow some of the sample to migrate into the stacking gel. Subsequently, the remainder of the sample was loaded into the appropriate well. Electrophoresis of the gel was then continued at 150 volts for a further 90 minutes, or until the blue marker dye had reached the bottom of the gel.

\subsubsection{Western Blotting}

A transfer sandwich was assembled to transfer the protein contained within the polyacrylamide gel onto a nitrocellulose membrane. All components, except the gel, were pre-soaked in transfer buffer (Appendix 2). The transfer sandwich components, in order from bottom to top, were: black face (negative charge), scotch pad, blotting paper, gel, nitrocellulose membrane, blotting paper, scotch pad, red face (positive charge). A roller was used to gently remove any air bubbles between each layer. The transfer sandwich was placed in the transfer stand along with a magnetic stirrer bar and ice pack, and transfer buffer was added to cover the transfer sandwich components. The transfer was performed at 100 volts for 70 minutes at $4{ }^{\circ} \mathrm{C}$. To check that the transfer was successful, the nitrocellulose membrane was incubated with Ponceau S stain (Appendix 2) for 1 minute at 60 rpm on a Labnet Orbit LS low speed platform shaker (Total Lab Systems Ltd.; Auckland, $\mathrm{NZ}$ ). The membrane was de-stained with $\mathrm{dH}_{2} \mathrm{O}$, and the position of the lanes was marked. The membrane was washed three times in Tris buffered saline (TBS; Appendix 2) before 
being blocked in $5 \%$ low-fat milk powder solution (Appendix 2) for 1 hour on the rotating platform shaker. The membrane was then incubated overnight in the appropriate primary antibody diluted in $5 \%$ low-fat milk powder solution on the rotating platform shaker. The primary antibodies are detailed in Section 2.4.5. The membrane was washed three times in TBS, before being incubated with a secondary antibody conjugated to horseradish peroxidise (HRP) in $5 \%$ low-fat milk powder solution. The secondary antibodies are detailed in Section 2.4.5. The membrane was washed three times in TBS, before being incubated for 1 minute in electrochemiluminescence ( $E C L$ ) reagents (Appendix 2). The membrane was wrapped in cling film and adhered to an exposure cassette. The $x$-ray films were exposed to the membrane for between 1 and 10 minutes, depending on the amount of protein signal. Following the development and fixture of the x-ray film, the ladder and lane positions were marked by comparison with the membrane.

\subsubsection{Western blot stripping}

The Western blots were stripped to remove the bound primary and secondary antibodies, allowing the membrane to be re-probed with different primary and/or secondary antibodies. The membrane was incubated in stripping buffer (Appendix 2) for 30 minutes at $50{ }^{\circ} \mathrm{C}$. The membrane was washed vigorously in TBS, before undergoing 3 further wash steps in TBS on a rotating platform shaker. Thereafter, the Western blot protocol was carried out from the 1 hour incubation step in low-fat milk powder solution.

\subsubsection{Antibodies}

A commercial anti-CX43 monoclonal antibody, MAB3067, was purchased from Millipore (Auckland, NZ). The MAB3067 antibody was raised in mice against the peptide sequence RSDPYHATTGPLSPSKDC, which corresponds to residues 252-270 of mouse CX43. This sequence is identical in rat CX43 and contains one potentially modified residue, a phosphoserine at residue 255 . The MAB3067 antibody was used at a final concentration of 1 $\mu \mathrm{g} / \mathrm{mL}$ in $5 \%$ low-fat milk powder solution. For ECL detection, blots that had been incubated with the MAB3067 antibody were subsequently incubated for 3 hours in $0.33 \mu \mathrm{g} / \mathrm{mL}$ rabbit anti-mouse secondary antibody conjugated to HRP (Jackson ImmunoResearch; Westgrove, PA, USA) in $5 \%$ low-fat milk powder solution. For blocking experiments, MAB3067 was preabsorbed overnight at $4{ }^{\circ} \mathrm{C}$ on a rotating wheel with $5 \mathrm{X}(\mathrm{w} / \mathrm{w})$ the corresponding control 
peptide (Millipore). The blocked antibody was centrifuged at $13,000 \times g$ for 10 minutes, and the supernatant was added to $5 \%$ low-fat milk powder solution.

The monoclonal antibody used to detect GDF9 (MAB47B) was produced by Oxford Brookes University for AgResearch. The MAB47B antibody was raised in mice against the peptide sequence SEYFKQFLFPQNEC, which corresponds to residues 122-135 (C-terminal region) of ovine GDF9. This sequence differs by one residue (bolded) in rat GDF9 (SEYFRQFLFPQNEC). Although the rat and sheep sequences are only $93 \%$ homologous, MAB47B has previously been shown to recognise rat GDF9 (Lin et al., 2012) and in this study was used at a concentration of $1 \mu \mathrm{g} / \mathrm{mL}$. For ECL detection, blots that had been incubated with MAB47B were subsequently incubated for 3 hours in $0.33 \mu \mathrm{g} / \mathrm{mL}$ rabbit anti-mouse secondary antibody conjugated to HRP. For blocking experiments, MAB47B was pre-absorbed overnight at $4{ }^{\circ} \mathrm{C}$ on a rotating wheel with $5 \mathrm{X}(\mathrm{w} / \mathrm{w}) \mathrm{E}$. coli produced recombinant ovine GDF9. The blocked antibody was centrifuged at $13,000 \times \mathrm{g}$ for 10 minutes, and the supernatant was added to $5 \%$ low-fat milk powder solution.

\subsubsection{Western blot analysis}

Quantitative analyses of the western blots were performed using ImageJ $1.45 \mathrm{~s}$ software. After scanning the x-ray film at high resolution, the scanned image was viewed in the ImageJ software package. The migration of the molecular standard was measured and used to estimate the size of the bands in the sample lanes. To measure the density of the bands, the image type was changed to 8-bit (grey-scale), and the subtract background process was used. The rectangular tool was used to select a band, and the measure function was used. The area of the rectangle was kept identical in all the corresponding bands of the same size. The integrated density (IntDen) value corresponds to the area and the mean grey value of the selection. For each band, the IntDen value was divided by the IntDen value of the time zero control. This value was subsequently divided by the relative amount of protein in each sample, as determined in the BCA assay. 


\section{VALIDATION OF A GJ BIOASSAY TO DETERMINE GJ ACTIVITY IN COC}

\subsection{Introduction}

GJ communication between the CC and oocyte is essential for the transfer of essential nutrients and maintenance of meiotic arrest. A novel bioassay utilising the properties of a fluorescent dye (calcein) for measuring GJ communication between CC and oocytes was first reported in cultured bovine COC in vitro (Thomas et al., 2004). This bioassay involves the passive entry of the acetoxymethyl derivate (calcein-AM) into cells via permeation of the cellular membrane. Once inside the cell, the acetoxymethyl group is cleaved by non-specific esterases rendering calcein as a membrane impermeable fluorescent compound. Thus, when calcein-AM is incubated with CC-enclosed oocytes, the majority ( $80 \%)$ of the fluorescent calcein measured within the oocyte has been transferred from the CC through GJ (Thomas et al., 2004). Indeed, treatment of bovine COC with carbenoxolone, a GJ blocking agent that disassembles GJ plaques and dephosphorylates GJ proteins (Guan et al., 1996; Cruciani and Mikalsen, 2002), inhibited calcein transfer to the oocyte in a dose dependent manner (Thomas et al., 2004).

Rat COC that have been isolated from their follicular environment resume meiosis within 1-2 hours under in vitro conditions (Wert and Larsen, 1989). Meiotic arrest in oocytes is maintained by high levels of CAMP and evidence suggests that COC extraction from follicles results in the breakage of GJ (Wert and Larsen, 1989) and a subsequent decline in intraoocyte CAMP concentrations. Studies in bovine COC indicate that the primary mechanisms that regulate cAMP levels in the oocyte are the production of cAMP by adenylyl cyclase in the CC followed by transfer to the oocyte, and the degradation of CAMP by PDE expressed in the oocyte (Thomas et al., 2002). Additionally, the degree of intracellular communication facilitated through GJ has been reported to be regulated by CAMP via protein kinase A (PKA)mediated phosphorylation (Cruciani and Mikalsen, 2002). Thus, maintenance of cAMP levels in the oocyte by the addition of adenylyl cyclase stimulators or PDE inhibitors during in vitro culture of $\mathrm{COC}$ is critical for maintaining GJ communication and meiotic arrest in oocytes. It has long been thought that the major PDE isoform expressed in the rodent oocyte is PDE3, while the major isoform expressed in rodent CC is PDE4D (Tsafriri et al., 1996; Richard et al., 2001). However, recent research has shown that inhibiting PDE8 activity in bovine COC 
increased cAMP levels and delayed oocyte nuclear maturation (Sasseville et al., 2009a). This suggests that species differences in the forms of PDE may exist.

Whilst the GJ bioassay had been modified for measuring GJ communication in rat COC (Patel, 2009; Yadav, 2010), the specificity of the assay and the effectiveness of a range of compounds involved in maintaining meiotic arrest and GJ communication has not been fully elucidated. Therefore, the aims of this chapter were to (1) determine the optimal in vitro conditions for maximising the rate of fluorescent dye transfer in rat $\mathrm{COC}$ and (2) demonstrate that fluorescent dye transfer from the CC to the oocyte is specific to GJ transfer and can be blocked using the GJ uncoupler carbenoxolone.

\subsection{Methodology}

\subsubsection{Tissue collection}

For experiments in sections 3.2.2-3.2.5 described below, ovaries were extracted and follicles were punctured in dissection media supplemented with $100 \mu \mathrm{M}$ milrinone. The COC were transferred into 48 -well plates containing wash media with $100 \mu \mathrm{M}$ milrinone. Due to the results obtained in these aforementioned sections, subsequent experiments utilised a modified collection methodology. Namely for experiments in sections 3.2.6-3.2.8, ovaries were extracted, follicles were punctured and COC were transferred into 48-well plates containing dissection media supplemented with $50 \mu \mathrm{M}$ dipyridamole and $50 \mu \mathrm{M}$ rolipram (see Sections 2.1.1 and 2.1.2 for detailed tissue collection methodology).

\subsubsection{Dose-response effects of DMSO concentrations}

To determine the effects of the solvent (DMSO) used to facilitate solubilisation of PDE inhibitors and/or treatments, milrinone was dissolved in differing volumes of DMSO to achieve a final milrinone concentration of $100 \mu \mathrm{M}$ and DMSO concentrations of $0.2 \%, 0.5 \%$, $1.0 \%$, and $2.0 \%$ in media. The COC were washed twice in wash media containing $100 \mu \mathrm{M}$ milrinone and the appropriate concentration of DMSO. The COC were then incubated with 2 $\mu \mathrm{M}$ calcein-AM in incubation media containing $100 \mu \mathrm{M}$ milrinone and the appropriate concentration of DMSO for 10 minutes. All incubations were carried out in an incubator at $37^{\circ} \mathrm{C}$ under $5 \% \mathrm{CO}_{2}$ in air with $96 \%$ humidity. Subsequently, the $\mathrm{COC}$ were transferred into incubation media containing $100 \mu \mathrm{M}$ milrinone and the appropriate concentration of DMSO without calcein-AM and incubated for either 0 or 4 hours. At the end of the incubation 
times, each COC was transferred into fresh incubation media (without milrinone or DMSO) where the oocyte was denuded and fluorescence was measured as described previously.

\subsubsection{Dose-response effects of forskolin}

Following collection, the $\mathrm{COC}$ were washed twice in wash media containing $100 \mu \mathrm{M}$ milrinone and incubated with $2 \mu \mathrm{M}$ calcein-AM in incubation media with $100 \mu \mathrm{M}$ milrinone for 10 minutes. To determine the effects of forskolin, the COC were then transferred to incubation media containing $100 \mu \mathrm{M}$ milrinone and $0,10,20$, or $50 \mu \mathrm{M}$ forskolin. After 0 or 4 hours incubation, the $\mathrm{COC}$ were transferred to fresh incubation media (no milrinone or forskolin) and the oocyte was denuded and fluorescence was measured as described previously (Section 2.1.3).

\subsubsection{Dose-response effects of single PDE inhibitors}

Three different PDE inhibitors were tested at the concentrations listed in Table 3-1. Milrinone is a specific inhibitor of PDE3 (Tsafriri et al., 1996), rolipram is a specific inhibitor of PDE4 (Sasseville et al., 2009a) and dipyridamole is reported to inhibit several PDE isoforms including PDE7, 8, 10, and 11 (Sasseville et al., 2009a). All concentrations of PDE inhibitors were dissolved in DMSO and the control media was supplemented with DMSO so that regardless of treatment, the final concentration of DMSO in media was $1 \%$.

Following collections, $\mathrm{COC}$ were washed twice in wash media without (control) or with the appropriate PDE inhibitor at the concentrations described above. The $\mathrm{COC}$ were incubated in $2 \mu \mathrm{M}$ calcein-AM without or with the appropriate PDE inhibitor for 10 minutes and then transferred into fresh incubation media (no calcein-AM) containing the appropriate PDE inhibitors or media alone (control). Following 0 or 4 hours incubation, the $\mathrm{COC}$ were transferred into incubation media alone (no PDE inhibitors) and the oocytes were denuded and fluorescence was measured as described previously (Section 2.1.3).

Table 3-1: Concentrations of PDE inhibitors used for the GJ dose-response experiments

\begin{tabular}{ccc}
\hline PDE inhibitor & PDE isoforms inhibited & Concentrations tested $(\boldsymbol{\mu} M)$ \\
\hline Milrinone & PDE3 & $100,300,500$ \\
Dipyridamole & PDE5, 6, 7, , $, 10,11$ & $10,50,100$ \\
Rolipram & PDE4 & $10,50,100$ \\
\hline
\end{tabular}




\subsubsection{Effects of combined PDE inhibitors}

Each PDE inhibitor was tested in combination with each other at the optimised concentration (as determined in section 3.2.4) as follows: $300 \mu \mathrm{M}$ milrinone alone, $50 \mu \mathrm{M}$ dipyridamole alone, $50 \mu \mathrm{M}$ rolipram alone, $300 \mu \mathrm{M}$ milrinone $+50 \mu \mathrm{M}$ dipyridamole, $300 \mu \mathrm{M}$ milrinone $+50 \mu \mathrm{M}$ rolipram, $50 \mu \mathrm{M}$ dipyridamole $+50 \mu \mathrm{M}$ rolipram, and $300 \mu \mathrm{M}$ milrinone + $50 \mu \mathrm{M}$ dipyridamole $+50 \mu \mathrm{M}$ rolipram. The final concentration of the DMSO used to solubilise the PDE inhibitors in the media was $1 \%$. Following collection, COC were washed twice in wash media without (control) or with the various PDE inhibitors as listed above. The COC were incubated for 10 minutes in $2 \mu \mathrm{M}$ calcein-AM in media alone (control) or with the appropriate PDE inhibitor(s). Thereafter, the COC were transferred to fresh incubation media (without calcein-AM) in the absence (control) or presence of PDE inhibitors. Following 0 or 4 hours incubation, $\mathrm{COC}$ were transferred into fresh media (free of PDE inhibitors) and the oocytes were denuded and the fluorescence intensity measured as described previously (Section 2.1.3).

\subsubsection{Effects of time on dye transfer to the oocyte}

Following collection, the $\mathrm{COC}$ were washed twice in wash media containing $50 \mu \mathrm{M}$ dipyridamole and $50 \mu \mathrm{M}$ rolipram. The $\mathrm{COC}$ were incubated in $2 \mu \mathrm{M}$ calcein- $\mathrm{AM}$ in incubation media containing $50 \mu \mathrm{M}$ dipyridamole and $50 \mu \mathrm{M}$ rolipram for 10 minutes. To determine the effect of incubation time on oocyte fluorescence, $\mathrm{COC}$ were transferred into fresh media (without calcein-AM) containing $50 \mu \mathrm{M}$ dipyridamole and $50 \mu \mathrm{M}$ rolipram and incubated for $0,1,2,3,4,12$, or 24 hours. Following incubation, $\operatorname{COC}$ were transferred into fresh incubation media containing no PDE inhibitors and the oocytes were denuded and the fluorescence intensity measured as described previously (Section 2.1.3).

\subsubsection{Testing the specificity of the GJ bioassay}

To determine the specificity of fluorescent dye transfer from the CC to the oocyte by GJs, COC were incubated with varying concentrations of the GJ uncoupler carbenoxolone. Following collection, $\mathrm{COC}$ were washed twice in wash media containing $50 \mu \mathrm{M}$ dipyridamole and $50 \mu \mathrm{M}$ rolipram. The $\mathrm{COC}$ were incubated for 10 minutes in $2 \mu \mathrm{M}$ calcein-AM in media containing $50 \mu \mathrm{M}$ dipyridamole and $50 \mu \mathrm{M}$ rolipram. COC were then transferred into fresh incubation media containing $50 \mu \mathrm{M}$ dipyridamole and $50 \mu \mathrm{M}$ rolipram and either $0,100,200$, 300,500 , or $1000 \mu \mathrm{M}$ carbenoxolone. Following 0 or 4 hours incubation, COC were 
transferred into incubation media free of PDE inhibitors and carbenoxolone and the oocytes were denuded and the fluorescence intensity measured as described previously (Section 2.1.3).

\subsubsection{Testing the robustness of the GJ bioassay}

To determine the robustness of the in vitro GJ bioassay over time, the proportion of fluorescence that entered the oocyte via a non-GJ pathway over time was assessed. Following collection, $\mathrm{COC}$ were washed twice in wash media containing $50 \mu \mathrm{M}$ dipyridamole and $50 \mu \mathrm{M}$ rolipram. The $\mathrm{COC}$ were transferred into incubation media containing $50 \mu \mathrm{M}$ dipyridamole and $50 \mu \mathrm{M}$ rolipram and incubated for $0,2,10$, or 22 hours. Those COC that were incubated for 2,10 , or 22 hours were then transferred to incubation media containing $1 \mathrm{mM}$ carbenoxolone, $50 \mu \mathrm{M}$ dipyridamole and $50 \mu \mathrm{M}$ rolipram and incubated for a further 2 hours, so the total culture times were 4,12 , or 24 hours, respectively. Following incubations, COC were exposed for 10 minutes to $2 \mu \mathrm{M}$ calcein-AM in incubation media containing $1 \mathrm{mM}$ carbenoxolone, $50 \mu \mathrm{M}$ dipyridamole and $50 \mu \mathrm{M}$ rolipram. The COC were incubated for 1 hour in calcein-AM free incubation media containing $1 \mathrm{mM}$ carbenoxolone, $50 \mu \mathrm{M}$ dipyridamole and $50 \mu \mathrm{M}$ rolipram. The $\mathrm{COC}$ were then transferred into incubation media without carbenoxolone or PDE inhibitors, the oocytes were denuded and fluorescence intensity measured as described previously (Section 2.1.3).

\subsubsection{Data Analysis}

The data were analysed as previously described in Section 2.1.3.1. Briefly, the oocyte fluorescence in each individual sample was corrected for the geometric mean of the control group (usually the untreated group at 0 hours). The data were analysed using the MannWhitney test, Kruskal-Wallis test with Dunn's multiple comparison post-hoc test, or a twoway ANOVA. Where a two-way ANOVA was used, the data were first tested for normality using the D'Agostino \& Pearson normality test. Data that was not normally-distributed was transformed using a natural log transformation, tested for normality again, and a two-way ANOVA was performed on the transformed data. 


\subsection{Results}

\subsubsection{Dose-response effects of DMSO concentrations}

The dose-response effects of DMSO in media on GJ communication in rat COC is depicted in Figure 3-1 where the fluorescent intensity values plotted are those at 4 hours normalised against the mean control value at 0 hours. There were no significant differences in relative oocyte fluorescent intensity at any of the DMSO concentrations tested $(P=0.055)$. Nonetheless whilst not significant, there appeared to be a reduction in intra-oocyte fluorescent intensity at the highest dose (2\%) of DMSO, thus the DMSO concentration of 1 $\%$ was used in all subsequent assays.

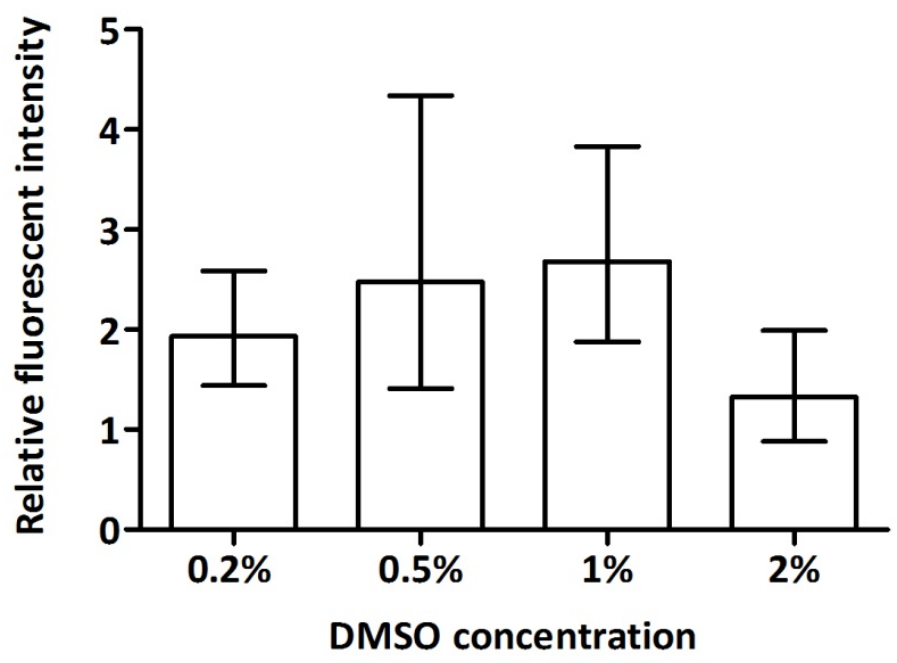

Figure 3-1: Effect of differing concentrations of DMSO on fluorescence transfer from adjacent CC to oocytes after a 4 hour incubation of rat COC. Values are shown as geometric means $\pm 95 \%$ confidence intervals of fluorescent ratios normalised against the geometric mean of the T0 control group (not shown, relative geometric mean=1.0). The data represent an $\mathrm{N}$ of 22-25 readings from individual oocytes in each group generated from 2 replicate experiments. The data were analysed using the Kruskal-Wallis test. No significant effect of DMSO concentration on relative fluorescent intensity was noted. The mean \pm SEM fluorescent reading in the control group at TO was $104 \pm 36$.

\subsubsection{Dose-response effects of forskolin}

The dose-response effects of forskolin (an adenylyl cyclase stimulator) on GJ communication in rat $\mathrm{COC}$ is shown in Figure 3-2, where the fluorescent intensity values plotted are those at 4 hours normalised against the mean control value at 0 hours. Incubation of COC for 4 hours 
with the two lowest doses of forskolin $(10 \mu \mathrm{M}$ or $20 \mu \mathrm{M})$ did not affect oocyte fluorescence. In comparison, incubation of $\mathrm{COC}$ for 4 hours with the highest dose of forskolin tested (50 $\mu \mathrm{M})$ resulted in a significant reduction $(\mathrm{P}<0.001)$ in oocyte fluorescence. The incubation time of 4 hours resulted in significantly increased $(P<0.0001$; Mann-Whitney test) fluorescent intensity in the control oocytes (no forskolin), compared to that at 0 hours (i.e. note the $\sim 4-6$ fold higher values; Figure 3-2A, B, \& C). The proportion of oocytes with a visible GV was similar between experimental treatment groups after 4 hours of incubation (Fisher's exact test; data not shown).

A

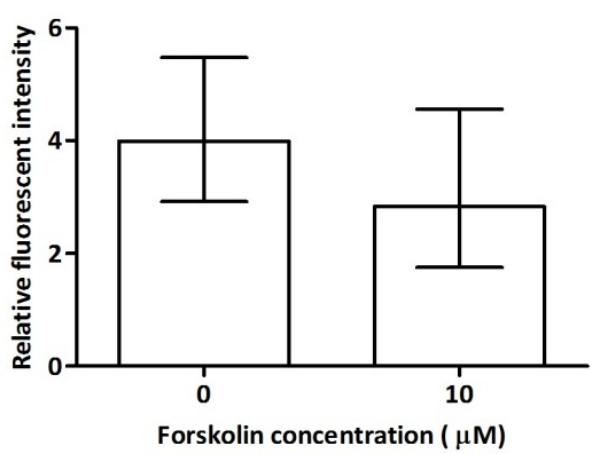

C

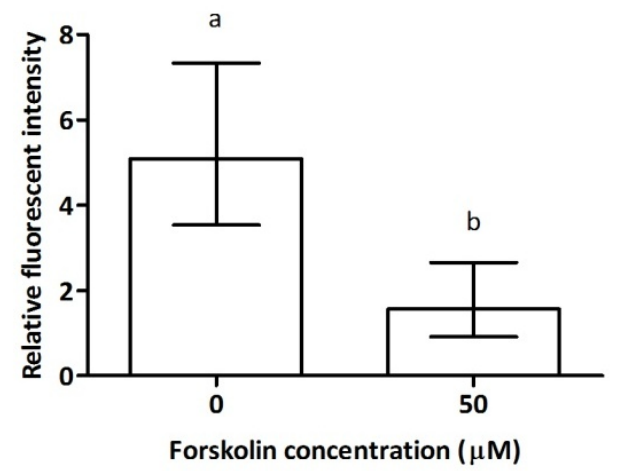

B

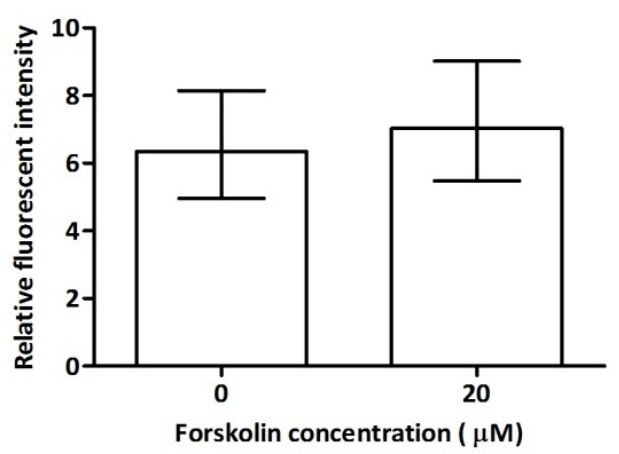

Figure 3-2: Effect of forskolin on fluorescent dye transfer from adjacent CC to oocytes after a 4 hour incubation of rat COC. Values are shown as geometric means $\pm 95 \%$ confidence intervals of fluorescent ratios normalised against the geometric mean of the T0 control group (not shown, relative geometric mean=1.0). The data represent an $N$ of (A) 46-64 readings from individual oocytes in each group generated from 5 replicate experiments, (B) 30-41 readings from individual oocytes in each group generated from 3 replicate experiments, (C) 8-19 readings from individual oocytes in each group generated from 1 replicate experiment. The data were analysed using the Mann Whitney test. No significant changes in relative fluorescent intensity were noted for $A$ or $B$. For $C$, groups that do not share a letter are statistically different $(P<0.05)$. The mean \pm SEM raw fluorescent reading in the control group at TO was (A) $56 \pm 18$, (B) $46 \pm 16$, and (C) $140 \pm 46$. 


\subsubsection{Dose-response effects of single PDE inhibitors}

The dose-response effects of milrinone (a PDE3 inhibitor) on GJ communication in rat COC is shown in Figure 3-3, where the fluorescent intensity values plotted are those at 4 hours normalised against the mean control value at 0 hours. There were no significant differences in oocyte fluorescent intensity following a 4 hour incubation of rat COC with 100 and $300 \mu \mathrm{M}$ of milrinone. In contrast, the highest dose of milrinone $(500 \mu \mathrm{M})$ resulted in a significant reduction $(P<0.01)$ in fluorescence compared to the other two dosages. The incubation time of 4 hours resulted in significantly increased ( $P<0.05$; Mann-Whitney test) fluorescent intensity in the control oocytes (no milrinone), compared to that at 0 hours (i.e. note the $\sim 2$ fold higher value; Figure 3-3). The proportion of oocytes with a visible GV, indicating arrested meiosis, was similar between all experimental treatment groups after 4 hours of incubation (Table 3-2).

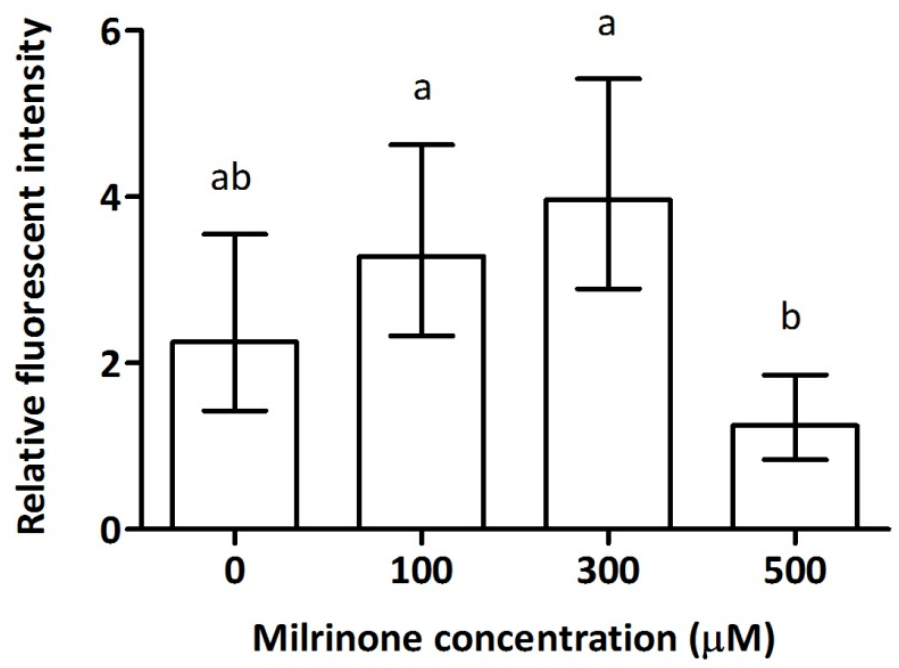

Figure 3-3: Effect of differing concentrations of milrinone on fluorescent dye transfer from adjacent CC to oocytes after a 4 hour incubation of rat COC. Values are shown as geometric means $\pm 95 \%$ confidence intervals of fluorescent ratios normalised against the geometric mean of the TO control group (not shown, relative geometric mean=1.0). The data represent 31-35 readings from individual oocytes in each group generated from 3 replicate experiments. The data were analysed by a Kruskal-Wallis test and where significant differences were observed, a Dunn's multiple comparison post-hoc test was performed. Groups that do not share a letter are statistically different $(P<0.05)$. The mean $\pm S E M$ raw fluorescent reading in the control group at TO was $206 \pm 28$. 
Table 3-2: Effect of differing concentrations of milrinone on the proportion of oocytes in which a GV was observed. The table shows the proportion (\%) of oocytes with a visible GV, with the actual number of oocytes with a GV observed in brackets. The data from 3 replicate experiments ( $N=31-35)$ were analysed by a Fisher's exact test. No significant effect of milrinone concentration on the presence of a GV was noted.

\begin{tabular}{cc}
\hline Milrinone concentration $(\mu \mathrm{M})$ & GV oocytes $(\%)$ \\
\hline 0 & $78(25)$ \\
300 & $94(33)$ \\
500 & $79(26)$ \\
\end{tabular}

The dose-response effects of dipyridamole (a PDE7, 8, 10, and 11 inhibitor) on GJ communication in rat $\mathrm{COC}$ is depicted in Figure 3-4, where the fluorescent intensity values plotted are those at 4 hours normalised against the mean control value at 0 hours. The incubation of $\mathrm{COC}$ with $50 \mu \mathrm{M}$ dipyridamole for 4 hours caused a significant increase in oocyte fluorescence relative to the appropriate control ( 4 hour incubation with the vehicle alone). No other concentration of dipyridamole tested resulted in a significant change in oocyte fluorescence relative to the control. The incubation time of 4 hours resulted in significantly increased $(P<0.01$; Mann-Whitney test) fluorescent intensity in the control oocytes (no dipyridamole), compared to that at 0 hours (i.e. note the $\sim 2$ fold higher value; Figure 3-4). The proportion of oocytes with a visible GV was similar between experimental treatment groups after 4 hours of incubation (Table 3-3). 


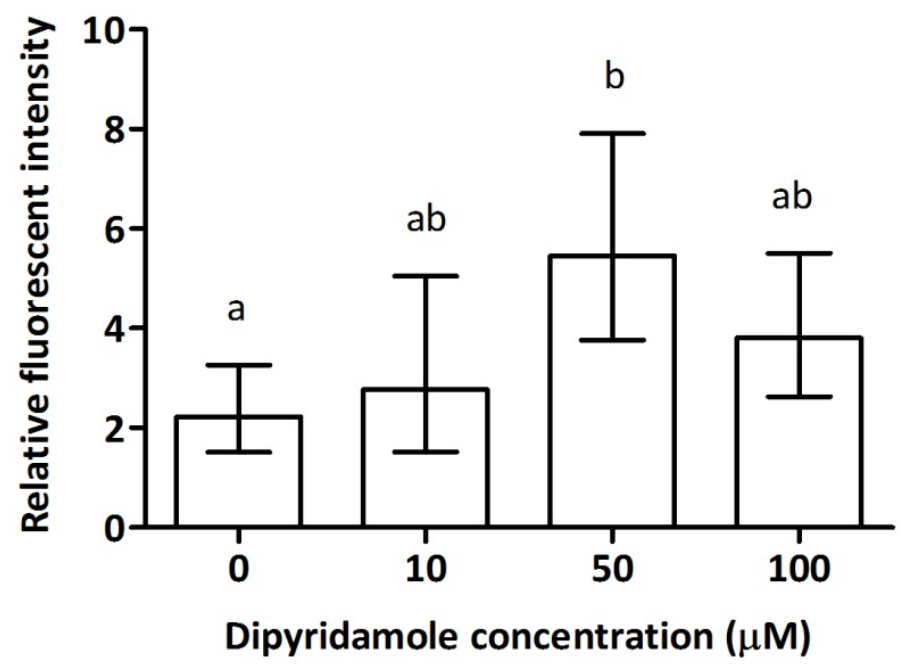

Figure 3-4: Effect of differing concentrations of dipyridamole on fluorescent dye transfer from adjacent CC to oocytes after a 4 hour incubation of rat COC. Values are shown as geometric means $\pm 95 \%$ confidence intervals of fluorescent ratios normalised against the geometric mean of the T0 control group (not shown, relative geometric mean=1.0). The data represent 34-38 readings from individual oocytes in each group generated from 4 replicate experiments. The data were analysed by a Kruskal-Wallis test and where significant differences were observed, a Dunn's multiple comparison post-hoc test was performed. Groups that do not share a letter are statistically different $(P<0.05)$. The mean \pm SEM raw fluorescent reading in the control group at T0 was $207 \pm 24$.

Table 3-3: Effect of differing concentrations of dipyridamole on the proportion of oocytes in which a GV was observed. The table shows the proportion (\%) of oocytes with a visible GV, with the actual number of oocytes with a GV observed in brackets. The data from 3 replicate experiments ( $N=34-38$ ) was analysed by a Fisher's exact test. No significant effect of dipyridamole concentration on the presence of a GV was noted.

\begin{tabular}{cc}
\hline Dipyridamole concentration $(\mu \mathrm{M})$ & GV oocytes $(\%)$ \\
\hline 0 & $59(22)$ \\
10 & $66(25)$ \\
50 & $81(29)$ \\
100 & $59(20)$ \\
\hline
\end{tabular}

The effect of incubating rat $\mathrm{COC}$ for 4 hours with differing concentrations of rolipram (a PDE4 inhibitor) on GJ communication is shown in Figure 3-5, where the fluorescent intensity values plotted are those at 4 hours normalised against the mean control value at 0 hours. No differences were observed in oocyte fluorescence between any experimental groups. The 
incubation time of 4 hours resulted in significantly increased $(P<0.005 ;$ Mann-Whitney test) fluorescent intensity in the control oocytes (no rolipram), compared to that at 0 hours (i.e. note the $\sim 2$ fold higher value; Figure 3-5). The proportion of oocytes with a visible GV was similar between experimental treatment groups after 4 hours of incubation (Table 3-4).

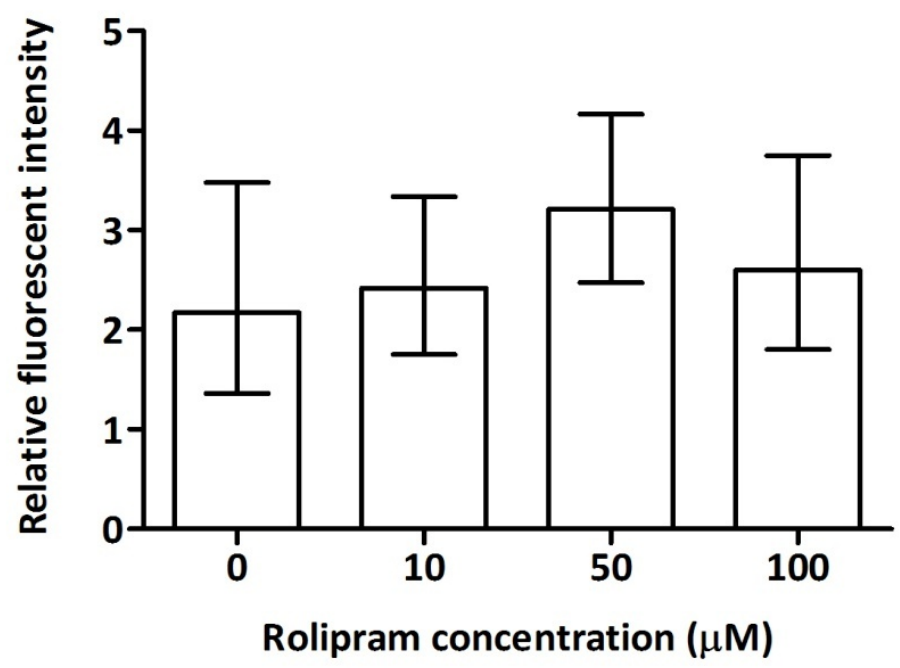

Figure 3-5: Effect of differing concentrations of rolipram on fluorescent dye transfer from adjacent CC to oocytes after a 4 hour incubation of rat COC. Values are shown as geometric means $\pm 95 \%$ confidence intervals of fluorescent ratios normalised against the geometric mean of the TO control group (not shown, relative geometric mean=1.0). The data represent 26-29 readings from individual oocytes in each group generated from 3 replicate experiments. The data were analysed by a Kruskal-Wallis test and where significant differences were observed, a Dunn's multiple comparison post-hoc test was performed. No significant changes in relative fluorescent intensity were noted. The mean \pm SEM raw fluorescent reading in the control group at T0 was $212 \pm 23$.

Table 3-4: Effect of differing concentrations of rolipram on the proportion of oocytes in which a GV was observed. The table shows the proportion (\%) of oocytes with a visible GV, with the actual number of oocytes with a GV observed in brackets. The data from 3 replicate experiments ( $N=26-29)$ was analysed by a Fisher's exact test. No significant effect of rolipram concentration on the presence of a GV was noted.

\begin{tabular}{cc}
\hline Rolipram concentration $(\mu \mathrm{M})$ & GV oocytes (\%) \\
\hline 0 & $32(12)$ \\
10 & $53(18)$ \\
50 & $43(17)$ \\
100 & $50(18)$ \\
\hline
\end{tabular}




\subsubsection{Effects of combined PDE inhibitors}

The effects of incubating rat $\mathrm{COC}$ for 4 hours with combinations of the aforementioned PDE inhibitors on GJ communication is shown in Figure 3-6, where the fluorescent intensity values plotted are those at 4 hours normalised against the mean control value at 0 hours. $A$ combination of dipyridamole and rolipram resulted in a significantly higher $(P<0.05)$ fluorescence intensity in oocytes compared to that in the untreated control group. All other treatment groups (PDE inhibitors alone and in combination) had oocyte fluorescent intensities similar to that of the control. The incubation time of 4 hours resulted in significantly increased ( $P<0.05$; Mann-Whitney test) fluorescent intensity in the control oocytes (no PDE inhibitors), compared to that at 0 hours (i.e. note the $\sim 2$ fold higher value; Figure 3-6). The proportion of oocytes with a visible GV was similar between all experimental treatment groups after 4 hours of incubation (Table 3-5).

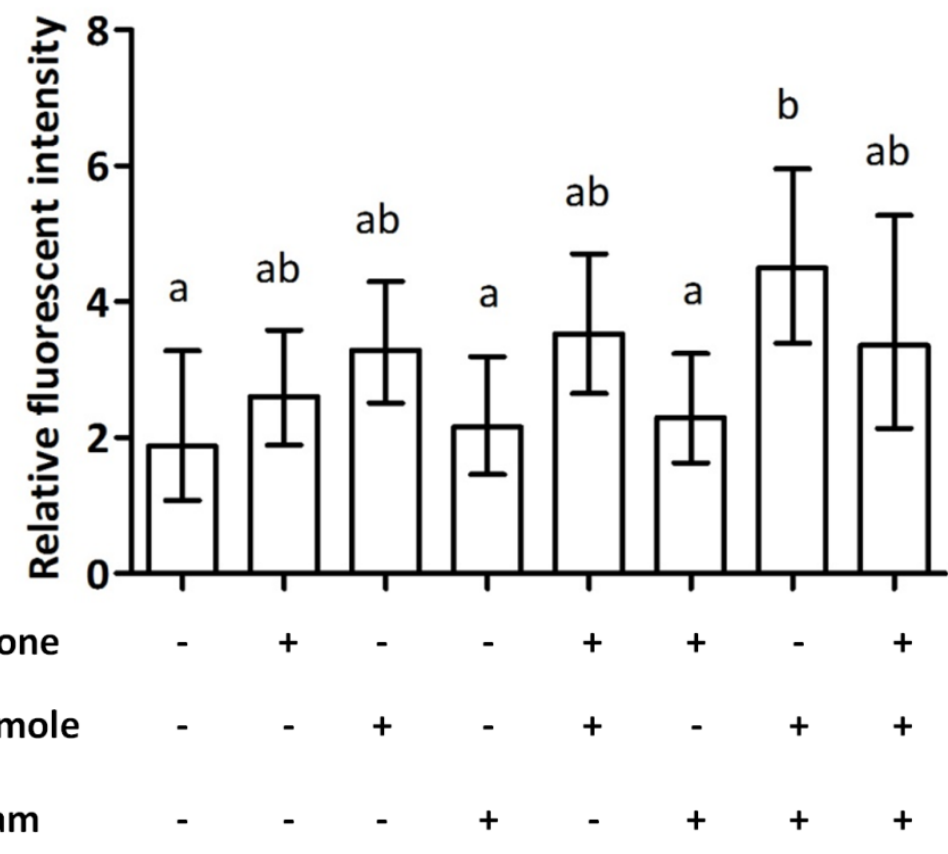

Figure 3-6: Effect of single or combinations of PDE inhibitors on fluorescent dye transfer from adjacent CC to oocytes after a 4 hour incubation of rat COC. Values are shown as geometric means $\pm 95 \%$ confidence intervals of fluorescent ratios normalised against the geometric mean of the TO control group (not shown, relative geometric mean=1.0). The data represent 22-30 readings from individual oocytes in each group generated from 3 replicate experiments. The data were analysed by a Kruskal-Wallis test and where significant differences were observed, a Dunn's multiple comparison post-hoc test was performed. Groups that do not share a letter are statistically different $(P<0.05)$. The mean \pm SEM raw fluorescent reading in the control group at T0 was $232 \pm 46$. 
Table 3-5: Effect of single or combinations of PDE inhibitors on the proportion of oocytes in which a GV was observed. The table shows the proportion (\%) of oocytes with a visible GV, with the actual number of oocytes with a GV observed in brackets. The data from 3 replicate experiments ( $N=22-30)$ was analysed by a Fisher's exact test. No significant effect of PDE inhibitor concentration on the presence of a GV was noted.

\begin{tabular}{cc}
\hline PDE inhibitor combination & \% GV oocytes \\
\hline No PDE inhibitors & $64(18)$ \\
Dipyridamone & $86(25)$ \\
Rolipram & $70(16)$ \\
Milrinone + Dipyridamole & $73(19)$ \\
Milrinone + Rolipram & $83(25)$ \\
Dipyridamole + Rolipram & $81(22)$ \\
Milrinone + Dipyridamole + Rolipram & $71(20)$ \\
\end{tabular}

\subsubsection{Effects of time on dye transfer to the oocyte}

The effect of varying the incubation time following exposure to calcein-AM on the fluorescent intensity of the oocyte is depicted in Figure 3-7. Initially, oocyte fluorescence increased with time to reach maximum levels after 2-3 hours of incubation. As a result of these experiments and to remain within a responsive fluorescent intensity range, COC were incubated for 1 hour after the 10 minute exposure to calcein-AM for all subsequent assays. 


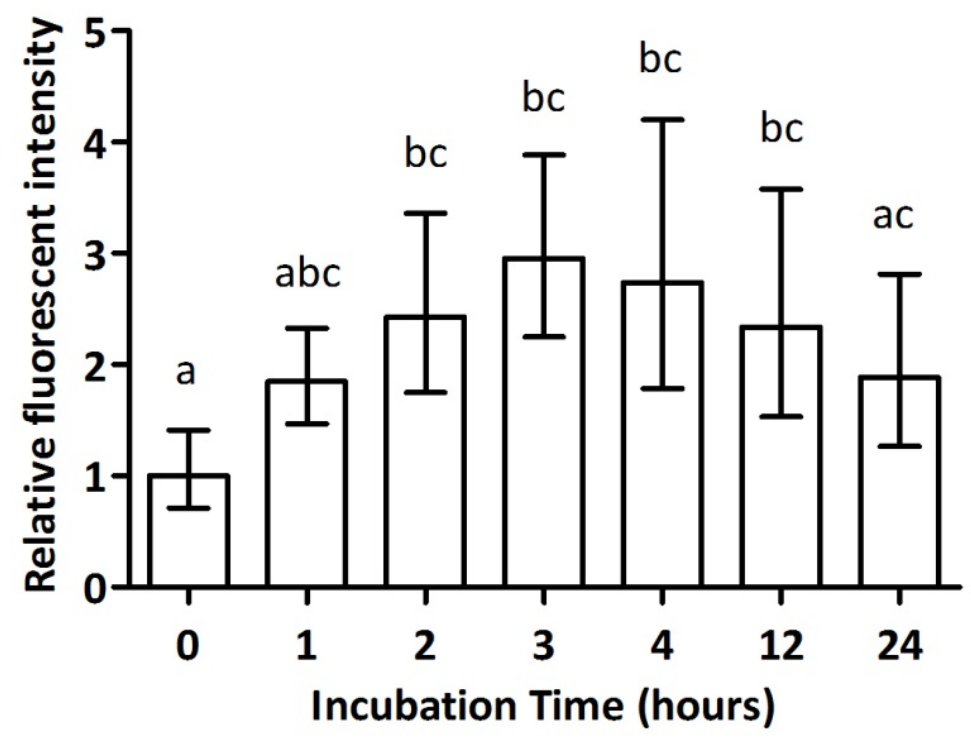

Figure 3-7: Effect of time on fluorescent dye transfer from adjacent CC to oocytes. Values are shown as geometric means $\pm 95 \%$ confidence intervals of fluorescent ratios normalised against the geometric mean of the T0 control group. The data represent 23-27 readings from individual oocytes at each time generated from 3 replicate experiments. The data were analysed by a Kruskal-Wallis test and where significant differences were observed, a Dunn's multiple comparison post-hoc test was performed. Groups that do not share a letter are significantly different $(\mathrm{P}<0.05)$. The mean \pm SEM raw fluorescent reading at T0 was $297 \pm 38$.

\subsubsection{Testing the specificity of the GJ bioassay}

To determine the proportion of calcein transfer to the oocyte that is facilitated through GJ, rat $\mathrm{COC}$ were exposed to increasing concentrations of the GJ blocking agent carbenoxolone. These results are depicted in Figure 3-8, in which the fluorescent intensity values plotted are those at 4 hours normalised against the mean control value at 0 hours. Carbenoxolone inhibited calcein transfer to the oocyte in a dose-dependent manner. The lowest concentration of carbenoxolone to cause a significant reduction ( 60\%; $\mathrm{P}<0.05)$ in oocyte fluorescence, compared to the control group, was $200 \mu \mathrm{M}$. The lowest dose that caused the maximum inhibition (>88\%; $\mathrm{P}<0.0001$ ) of $\mathrm{GJ}$ communication within $\mathrm{COC}$ was $500 \mu \mathrm{M}$ carbenoxolone. The incubation time of 4 hours resulted in significantly increased $(P<0.0001$; Mann-Whitney test) fluorescent intensity in the control oocytes (no carbenoxolone), compared to that at 0 hours (i.e. note the $\sim 4$-fold higher value; Figure 3-8). 


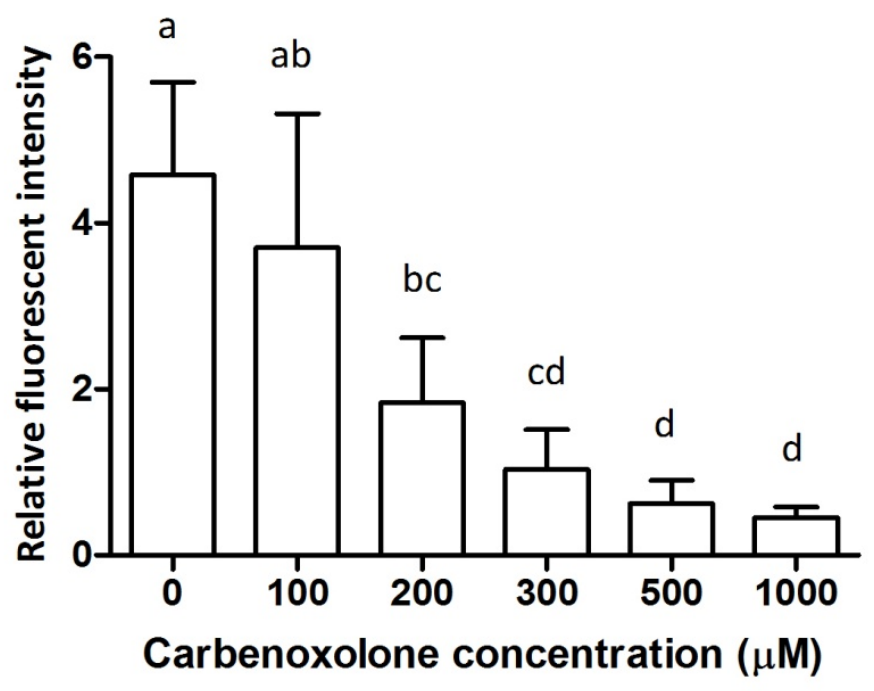

Figure 3-8: Effect of increasing concentrations of carbenoxolone on fluorescent dye transfer from adjacent CC to oocytes after a 4 hour incubation of rat COC. Values are shown as geometric means $\pm 95 \%$ confidence intervals of fluorescent ratios normalised against the geometric mean of the T0 control group (not shown, relative geometric mean $=1.0$ ). The data represent 25-37 readings from individual oocytes in each group generated from 4 replicate experiments. The data were analysed by a Kruskal-Wallis test and where significant differences were observed, a Dunn's multiple comparison post-hoc test was performed. Groups that do not share a letter are significantly different $(P<0.05)$. The mean $\pm S E M$ raw fluorescent reading in the control group at TO was $195 \pm 35$.

\subsubsection{Testing the robustness of the GJ bioassay}

The robustness of the in vitro GJ bioassay over time was assessed by determining the proportion of fluorescent dye that enters the oocyte via a means other than GJs over time. These results are depicted in Figure 3-9, in which the fluorescent intensity values plotted are normalised against the mean control value at 0 hours. As carbenoxolone takes at least 2 hours to effectively block GJ (see Appendix 1), the specificity of calcein transfer to the oocyte in the COC incubated for 1 hour was not able to be determined. A 2 hour incubation of COC with carbenoxolone, regardless of a prior 2, 10, or 22 hour incubation without carbenoxolone, caused a marked decrease (91\%, $85 \%$, and $92 \%$, respectively) in dye transfer to the oocyte by the end of the total incubation period. All of the incubation times tested resulted in significantly increased $(\mathrm{P}<0.0001$; Mann-Whitney test) fluorescent intensities in the control oocytes, compared to those at 1 hours (i.e. note the $\sim 3.5$-fold higher values, Figure 3-9). 


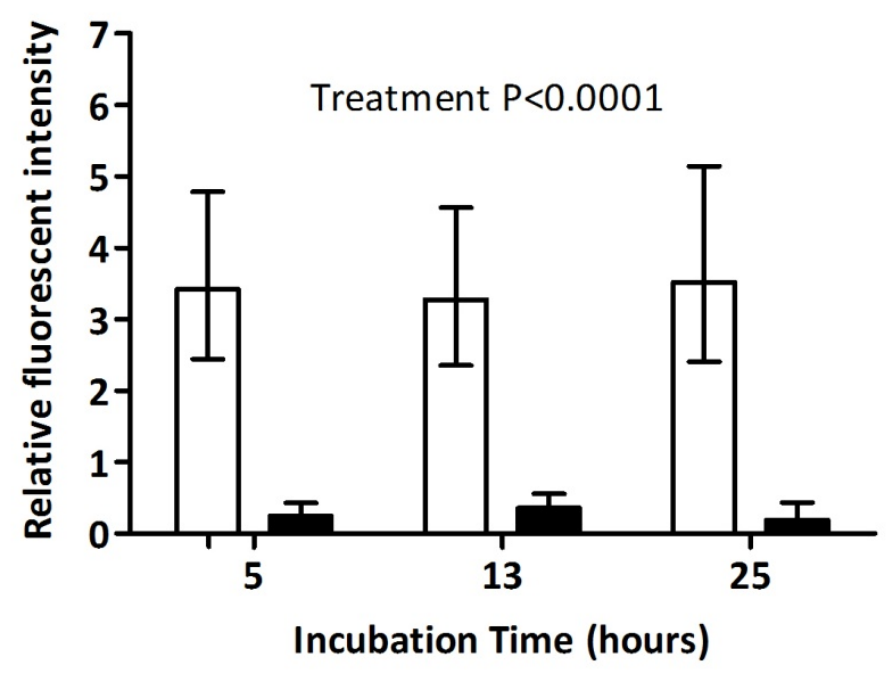

Figure 3-9: Effect of $1 \mathrm{mM}$ carbenoxolone on fluorescent dye transfer from adjacent CC to oocytes after 5, 13, and 25 hours incubation in rat COC. The incubation time indicates the pre-calcein incubation + the 1 hour postcalcein incubation. The control (i.e. untreated) group is represented by the unfilled bars ( $\square$ ), while the carbenoxolone-treated group is represented by the filled bars ( $)$. Values are shown as geometric means \pm $95 \%$ confidence intervals of fluorescent ratios normalised against the geometric mean of the control group at 1 hour (not shown, relative geometric mean $=1.0$ ). The data represents $23-44$ readings from individual oocytes in each group generated from 3 replicate experiments. The natural log-transformed data were analysed by a two-way ANOVA. The mean \pm SEM raw fluorescent reading in the control group at 1 hour was $112 \pm 14$.

\subsection{Discussion}

These validation studies reveal for the first time in the rat that, of all the cAMP-modulating compounds tested the combination of dipyridamole and rolipram was arguably the most effective in maintaining GJ communication between the CC and oocyte. Interestingly, the inhibitors milrinone and rolipram that block the PDE isoforms reported to be primarily expressed in rat oocytes (i.e. PDE3; Richard et al., 2001) and CC (i.e. PDE4; (Tsafriri et al., 1996), respectively had no effect on GJ communication in this study either singly or in combination with one another. Dipyridamole is reported to inhibit the actions of PDE7, 8, 10, and 11 (Sasseville et al., 2009a). Whilst the expression of PDE8 has previously been detected in the rat ovary (Kobayashi et al., 2003), PDE8 activity on regulating cAMP concentrations or oocyte meiotic resumption in this species has not been reported. Additionally, to the best of my knowledge, the expression of PDE7, 10, and 11 in the rat COC has not been previously detected. Therefore, these data indicate for the first time that the levels of PDE7, 8, 10, and/or 11 are active in rat COC. In the cow, PDE8 has been reported to account for up to 20 $\%$ and $60 \%$ of PDE activity in oocytes and CC, respectively. If a similar relationship exists in 
the rat $\mathrm{COC}$, these results suggest that inhibition of PDE activity in the $\mathrm{CC}$, rather than the oocyte, is more important for maintaining GJ communication in this species. These results also suggest that prevention of CAMP breakdown (tested by the addition of PDE inhibitors), rather than stimulation of cAMP production (tested by the addition of forskolin) is more important for maintaining GJ communication in the rat.

The ability of the GJ inhibitor carbenoxolone to inhibit calcein transfer to the oocyte up to 25 hours in culture indicates that these culturing conditions allow the $\operatorname{COC}$ to retain its structural integrity in vitro. Furthermore, calcein transfer from the $\mathrm{CC}$ to the oocyte was reduced in a dose-dependent manner with increasing concentrations of carbenoxolone. Maximum inhibition ( $90 \%$ ) of fluorescence transfer from the CC to the oocyte was achieved at a dose of $500 \mu \mathrm{M}$ carbenoxolone. Thus, approximately $10 \%$ of fluorescence measured within DO using the bioassay was the result of transfer facilitated by a means other than GJ. Indeed, other transfer channels exist in oocytes including aquaporins and calcium channels (Ford et al., 2000; Tosti et al., 2000). Aquaporins are involved in the transfer of water and neutral solutes such as polyols, purines, pyrimidines, and carbamides, and are thought to mediate oocyte volume control (Tsukaguchi et al., 1998; Ford et al., 2000). Calcium channels are thought to play a role in oocyte meiotic resumption (Tosti, 2006).

The optimal parameters for measuring GJ communication in rat $\mathrm{COC}$ using the bioassay herein include the addition of $50 \mu \mathrm{M}$ dipyridamole and $50 \mu \mathrm{M}$ rolipram in media. The inclusion of these PDE inhibitors in media should be used for ovary collections and dissections, throughout all in vitro incubation periods and the 1 hour incubation time following calcein-AM exposure to allow for appropriate dye transfer. Furthermore, the final concentration of the solvent DMSO should remain at $<1 \%$.

In summary, we report a successful bioassay for measuring GJ communication in rat COC that utilises a novel combination of PDE inhibitors (dipyridamole and rolipram). This study infers that PDE7, 8, 10 and 11 are active in the rat COC, and may play an important role in the maintenance of meiotic arrest in the oocyte in this species. Moreover this study verifies that GJ communication within the rat $\mathrm{COC}$ is a dynamic process that the bioassay described herein can accurately and repeatedly measure. 


\section{VALIDATING THE USE OF COC PREVIOUSLY UTILISED IN GJ ASSAYS FOR GENE EXPRESSION ANALYSES}

\subsection{Introduction}

Ovarian follicles are activated to grow one at a time such that each one is at a different stage of development at any one time. Even when a large number of pre-ovulatory follicles are produced in sheep through hormonal superovulation protocols, it has been shown that GC isolated from these similarly-sized follicles showed significant differences in their responses to FSH, and hCG (McNatty et al., 2010). Thus, superovulation protocols are unable to overcome the hierarchical growth of ovarian follicles. Moreover, large differences in oocyte quality have also been reported following superovulation in humans (Krisher, 2004; Lasien et al., 2009) confirming that similar-sized follicles are unlikely to be at an identical stage of development, or to respond identically to external stimuli. These observations highlight the

importance of assessing both the degree of GJ communication and the expression levels of candidate genes in individual COC. Moreover, the ability to directly correlate the degree of GJ communication and candidate gene expression levels would be invaluable for assessing the regulatory pathway(s) that are involved in CC-oocyte interactions.

In order to directly correlate CC and oocyte gene expression with GJ activity, the aim of this study was to determine whether (1) the fluorescent properties of the dye (calcein) used in the GJ assays interfered with the fluorescence of the TaqMan probes used in the qPCR technique and (2) the exposure of COC to calcein affected mRNA levels of the candidate genes.

\subsection{Methods}

For all experiments described below, the fluorescence was measured in a Corbett RotorGene 6000 machine following a thermal cycle programme of: $95^{\circ} \mathrm{C}$ for 10 minutes; and then 40 cycles of $95{ }^{\circ} \mathrm{C}$ for 15 seconds and $60{ }^{\circ} \mathrm{C}$ for 60 seconds. The Corbett was set to acquire florescence on the green, orange, red, and yellow channels. The excitation and emission wavelengths for these channels (Table 4-1) determine the four florescence dyes chosen for the tags on the TaqMan probes, namely FAM, ROX, CY5 and HEX, respectively. Calcein has excitation and emission wavelength of 495 and $515 \mathrm{~nm}$, respectively. 
Table 4-1: Excitation and emission wavelengths for the green, orange, red, and yellow channels of the Corbett Rotor Gene 6000

\begin{tabular}{ccc}
\hline Channel & Excitation $(\mathbf{n m})$ & Emission $(\mathbf{n m})$ \\
\hline Green (FAM) & 470 & 510 \\
Orange (ROX) & 585 & 610 \\
Red (CY5) & 625 & 660 \\
Yellow (HEX) & 530 & 555 \\
\hline
\end{tabular}

\subsubsection{Detection of calcein fluorescence in qPCR reactions}

To determine whether the fluorescence emitted by calcein in a diluted solution was detected by the Corbett apparatus, a $1 \mathrm{mM}$ calcein-AM stock was diluted to a concentration of $40 \mu \mathrm{M}$ in $\mathrm{dH}_{2} \mathrm{O}$. Fluorescence was subsequently measured using the Corbett under the thermal conditions described above.

To determine whether fluorescence in DO subjected to a GJ assay (i.e. exposed to calceinAM) was detectable by the Corbett apparatus, $\mathrm{COC}$ were extracted and included in a routine GJ assay. In brief, COC were incubated with incubation media supplemented with $50 \mu \mathrm{M}$ dipyridamole and $50 \mu \mathrm{M}$ rolipram and in the absence (control) or presence (treated) of $2 \mu \mathrm{M}$ calcein-AM for 10 minutes. The COC were then transferred into calcein-AM free incubation media containing $50 \mu \mathrm{M}$ dipyridamole and $50 \mu \mathrm{M}$ rolipram and incubated for a further 4 hours. The CC were removed from the oocyte and fluorescence was measured within each individual oocyte as described previously (see Section 2.1 .3 ). The oocytes were stored at -80 ${ }^{\circ} \mathrm{C}$ until further use. To determine whether fluorescence from the calcein is detected in cDNA of oocytes that were previously subjected to the GJ assay procedure, RNA was extracted from some of the oocytes collected in the previous experiment and CDNA synthesised. Fluorescence was detected using the Corbett under the thermal conditions described previously.

\subsubsection{Correlation of Rpl19 $C_{T}$ value to CC number and oocyte diameter}

To test the relationship between CC number and Rp/19 expression levels, COC were extracted from ovaries into PBS as described previously. The oocytes were stripped of the CC by vigorous pipetting and the oocytes were discarded from the resulting cell suspension. The number of $\mathrm{CC} / \mathrm{mL}$ was determined by counting the $\mathrm{CC}$ under an inverted microscope using a 
haemocytometer and Trypan blue stain (Section 2.1.5). The CC were serially diluted in triplicate, and stored at $-80{ }^{\circ} \mathrm{C}$ prior to extraction of total RNA and synthesis into CDNA. The level of Rp/19 mRNA was ascertained in each sample in duplicate using TaqMan qPCR.

To test the relationship between oocyte diameter and $R p / 19$ expression levels, oocytes with no CC contamination were selected from the experiment in Section 4.2.3. The diameter of each oocyte was measured, and this was compared to the level of Rp/19 mRNA measured using multiplex TaqMan qPCR.

\subsubsection{Effect of calcein/calcein-AM on expression of candidate genes}

To test whether exposure to calcein-AM during the GJ assay affects the expression levels of the candidate genes, a GJ assay was performed in which the COC were, or were not, exposed to calcein-AM. The COC were extracted from ovaries as described previously, and incubated either for $0,4,12$, or 24 hours in incubation media containing $50 \mu \mathrm{M}$ dipyridamole and 50 $\mu \mathrm{M}$ rolipram. The $\mathrm{COC}$ were further incubated for 10 minutes in the aforementioned incubation media in the absence (control) or presence (treated) of $2 \mu \mathrm{M}$ calcein-AM. The $\mathrm{COC}$ were transferred into fresh incubation media (same as above) but without calcein-AM and incubated for a further 1 hour. The oocyte was denuded of CC, and oocyte fluorescence was measured as described previously. Individual CC masses and DO were collected separately in PBS and stored at $-80{ }^{\circ} \mathrm{C}$ until further use.

Total RNA was extracted from the thawed CC and oocyte samples, and CDNA was synthesised as previously described (see Section 2.3.1). Multiplex TaqMan qPCR was performed to determine the mRNA levels of the following sets of genes: Atr, Cycs, Glut1 and Rpl19 in DO and CC separately; Cx43, Gfpt1, Pfkp and Rp/19 in CC; and Gdf9, Bmp15, Cx37 and Rpl19 in DO. The data were analysed using the $2^{-\left(\Delta \Delta C_{T}\right)}$ method (Section 2.3.5; Livak and Schmittgen, 2001). As discussed herein, CC-derived genes were corrected for the reference gene $R p / 19$ to give gene expression relative to input, while oocyte-derived genes were corrected to an internal calibrator control to give total gene expression in a single oocyte. The relative linear mRNA levels were analysed for normality using the D'Agostino \& Pearson normality test. Data that were not normally-distributed were transformed (natural log, $1(y+1)$, or square $\operatorname{root}(y+0.5)$ transformations) and reanalysed using the D'Agostino \& Pearson normality test to confirm a normal distribution. Normally-distributed data were 
analysed by a two-way ANOVA. In cases where data were not normally-distributed after all transformation options were exhausted, the data were ranked and a two-way ANOVA was performed on the ranked values.

\subsection{Results}

\subsubsection{Detection of calcein fluorescence}

The fluorescence levels detected in a $40 \mu \mathrm{M}$ calcein-AM solution is shown in Figure 4-1. Fluorescence was detected at very high levels on the green channel but was not detected above baseline levels on the orange, red, or yellow channels.
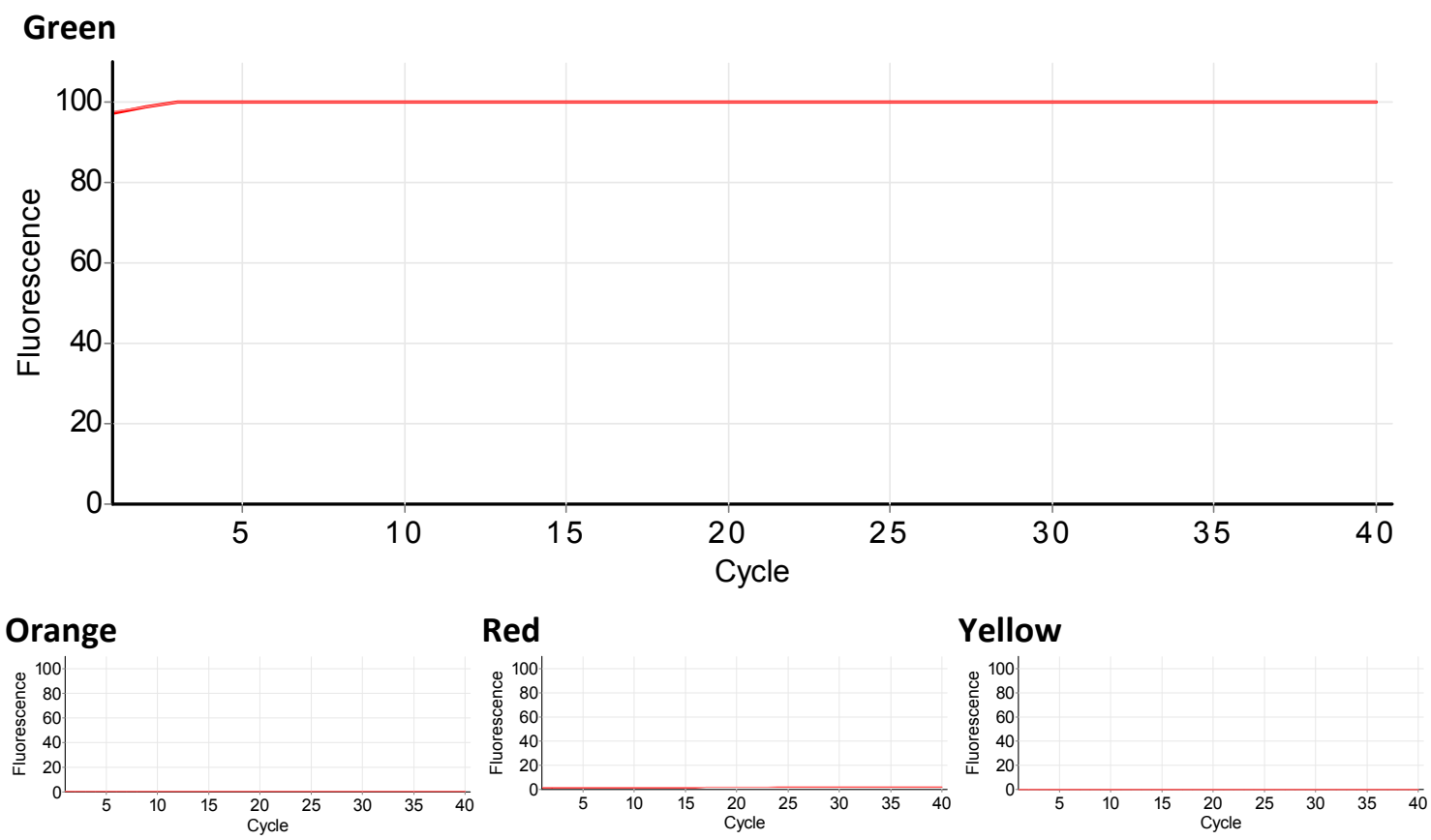

Figure 4-1: Detection of fluorescence in a $\mathbf{4 0} \mu \mathrm{M}$ calcein-AM solution over $\mathbf{4 0}$ cycles. The red lines show, in duplicate, the fluorescence measured on each channel in the calcein-AM solution.

The fluorescence levels detected in the lysate of individual oocytes that had been incubated in the absence or presence of calcein-AM is shown in Figure 4-2. Fluorescence was not detected above the baseline levels on any channel in either the control or treated oocytes. 


\section{Green}

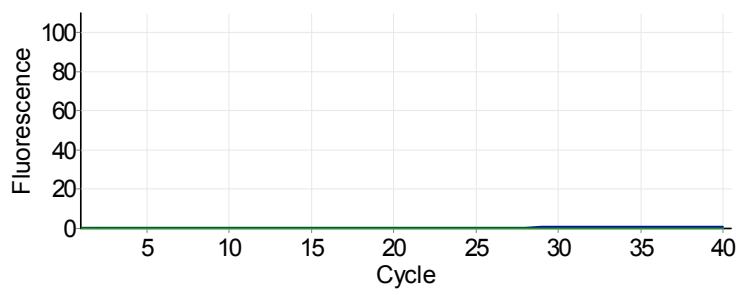

Red

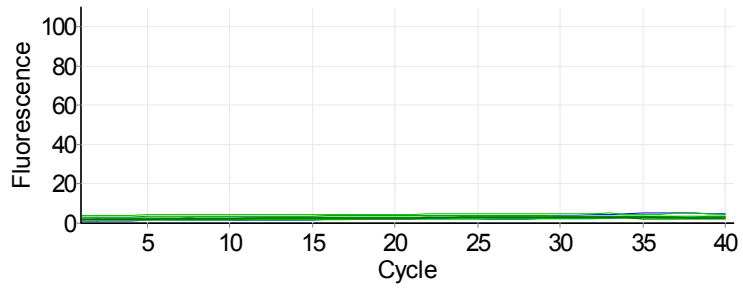

Orange

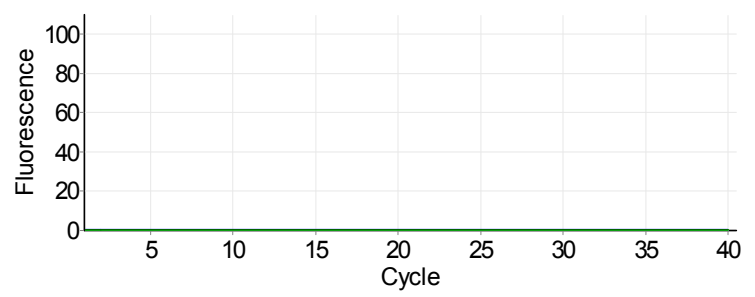

Yellow

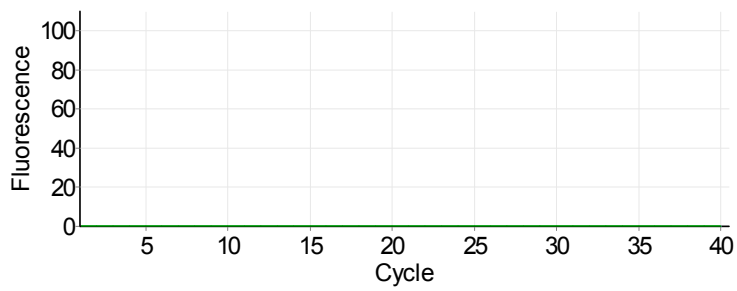

Figure 4-2: Detection of fluorescence in DO that had been incubated with and without calcein over $\mathbf{4 0}$ cycles. The blue lines show, in duplicate, a pool of DO that had not been treated with calcein $(\mathrm{N}=1)$. The green lines show, in duplicate, the DO that had been treated with calcein $(\mathrm{N}=8)$.

The fluorescence levels detected in CDNA of DO that had been incubated in the absence or presence of calcein-AM is shown in Figure 4-3. Fluorescence was not detected above baseline levels on any channel for cDNA from either the control or treated oocytes.

\section{Green}

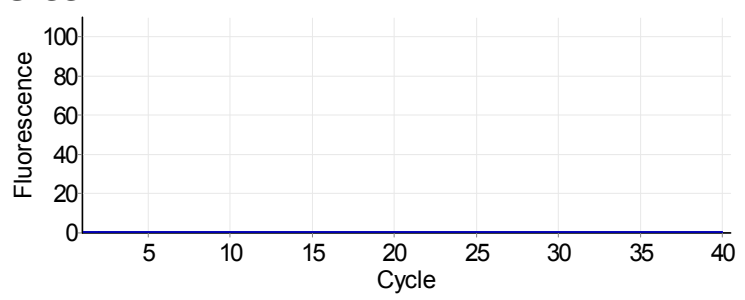

Red

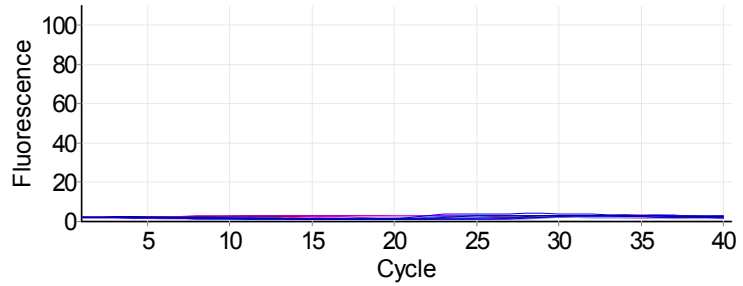

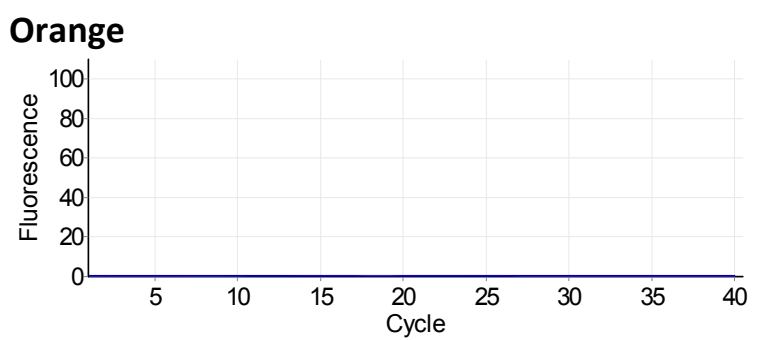

Yellow

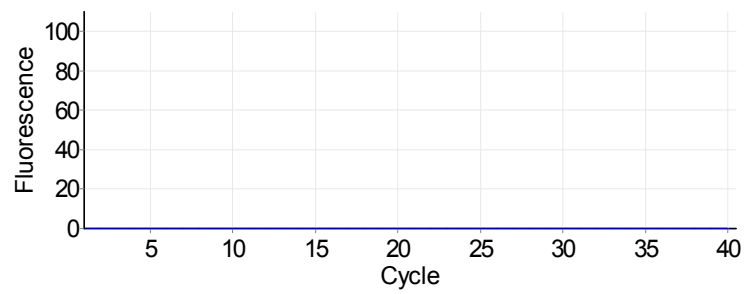

Figure 4-3: Detection of fluorescence in cDNA samples of oocytes that had been incubated with and without calcein over $\mathbf{4 0}$ cycles. The purple lines show, in duplicate, cDNA from oocytes that were not treated with calcein $(\mathrm{N}=3)$. The blue lines show, in duplicate, cDNA from oocytes that were treated with calcein $(\mathrm{N}=5)$. 


\subsubsection{Correlation of Rpl19 $C_{T}$ value to CC number and oocyte diameter}

CC were collected, counted, and serially diluted to determine the relationship between CC number and Rpl19 expression levels as denoted by $C_{T}$ values. This is depicted in Figure 4-4A. Expression levels of Rp/19 were highly correlated to the number of CC. The minimum number of cells required to measure $R p / 19$ (i.e. the $C_{T}$ value at the upper sensitivity limit $=$ 30) was 900.

The relationship between oocyte diameter and Rp/19 expression levels as denoted by $C_{T}$ values is shown in Figure 4-4B. There was no relationship between oocyte diameter and Rpl19 expression levels. The average oocyte diameter was $74 \mu \mathrm{M}$ (range 58-95 $\mu \mathrm{M}$ ).

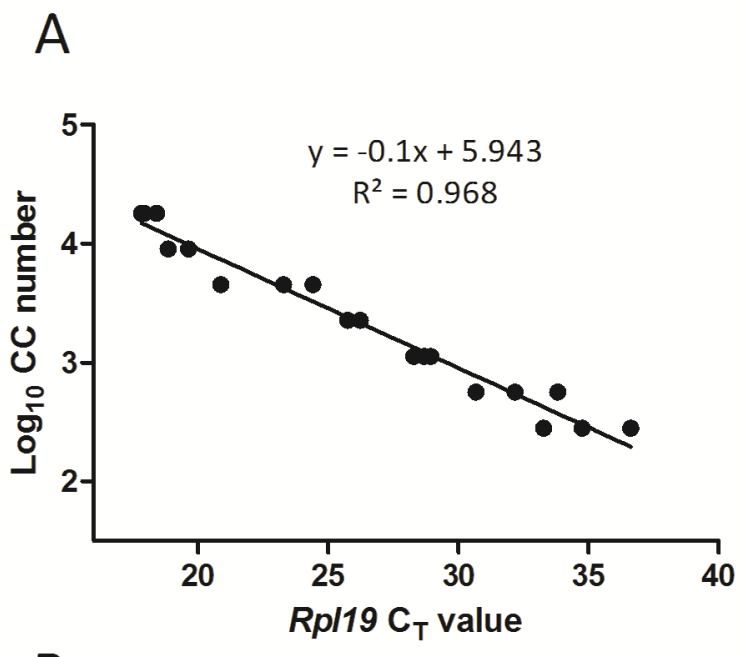

B

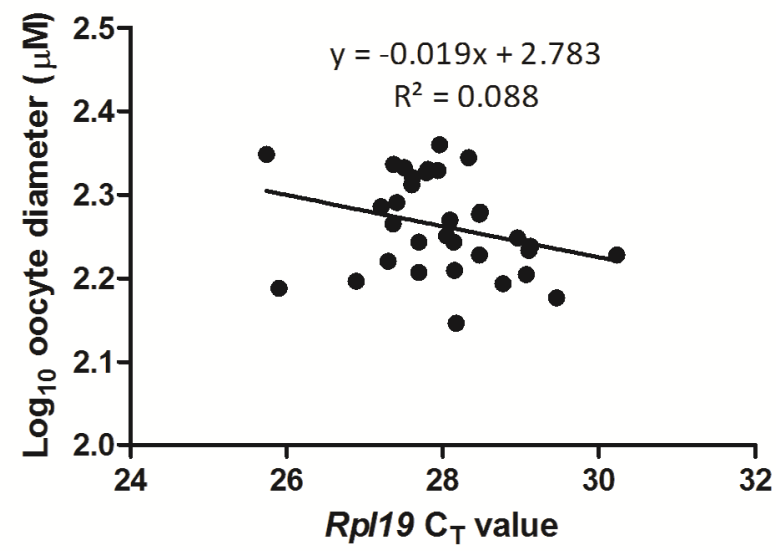

Figure 4-4: Correlation between $R p / 19 \mathrm{C}_{\mathrm{T}}$ value and CC number (A) or oocyte diameter (B). CC were serially diluted and the Rp/19 levels were measured using TaqMan qPCR. The Rp/19 mRNA levels in single oocytes were measured using TaqMan qPCR and compared to the diameter of each oocyte. 


\subsubsection{Effect of calcein on gene expression}

The effect of calcein-AM treatment with time on expression of the reference gene Rp/19 in DO and CC masses is shown in Figure 4-5. The average number of CC collected from each COC was 1495 (range 988-2397). Levels of Rp/19 mRNA in the oocyte and CC decreased over time in both the untreated and calcein-AM treated groups. No effect of calcein-AM treatment was measured for Rp/19 mRNA levels in the oocyte or the CC. A treatment $\mathrm{x}$ time interaction effect for Rp/19 mRNA levels in CC, but not oocytes, approached significance $(P=0.058)$.

Some of the oocyte samples were contaminated with CC due to improper denuding technique. When those oocytes that had >5 CC attached were excluded from the analysis, the effect of time on $R p / 19$ levels remained $(P<0.05)$, but no treatment or treatment $\mathrm{x}$ time interaction effects were noted.

For future gene expression studies only the 1 and 25 hour incubation times were used. When the 5 and 13 hour times were excluded from the analysis of Rp/19 levels in the oocyte, the effect of time remained $(P<0.0001)$ and no effects due to treatment or a treatment $x$ time interaction were observed. When the 5 and 13 hour times were excluded from the analysis of Rp/19 levels in the CC, a treatment $\mathrm{x}$ time interaction effect was measured $(P<0.01)$ but no effects of treatment or time were observed. 


\section{A}

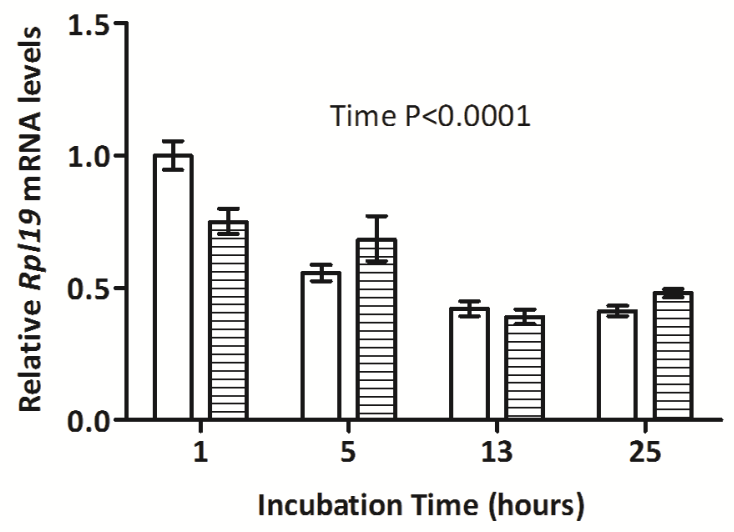

B

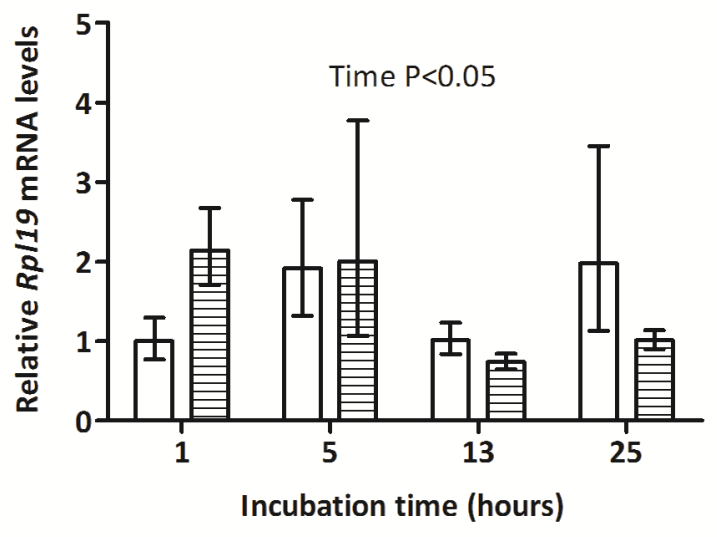

Figure 4-5: Effect of calcein-AM with time on Rp/19 expression levels in oocytes (A) and CC (B). The incubation time indicates the pre-calcein incubation + the 1 hour post-calcein incubation. The control (i.e. not exposed to calcein-AM) is represented by the unfilled bars ( $\square$ ), while the calcein-AM treated group is represented by the hatched bars ( oocyte sample was an average from the two oocyte gene quadriplex sets (Atr/ Cycs/Glut1/Rpl19 and Gdf9/Bmp1/CX37/Rpl19). The mRNA value of Rp/19 for each CC sample was an average from the two CC gene quadriplex sets (Atr/Cycs/Glut1/Rp/19 and CX43/Gfpt1/Pfkp/Rp/19). Values are shown as means \pm SEM analysed from linearised gene expression values normalised against the mean of the control group at 1 hour, which had a mean of 1 . The data represent 6-15 individual samples in each group generated from 3 replicate experiments. A two-way ANOVA was performed on natural log-transformed data.

The effects of calcein-AM treatment on the expression levels of candidate genes of interest for the oocyte (i.e. Atr, Gdf9, Bmp15, and Cx37) are shown in Figure 4-6. Oocyte-derived expression levels of Cycs and Glut1 were also tested, but were below the detectable limit. A significant effect of time was observed for Atr mRNA levels, with Atr levels decreasing over time in both the control and calcein-AM treated oocytes. No effect of calcein-AM treatment was observed for Atr mRNA levels. A significant effect of time was observed for Bmp15 levels 
in rat oocytes. Overall Bmp15 mRNA levels decreased over time. Bmp15 levels were similar between the control and calcein-AM treated groups at all time points except at 13 hours. At 13 hours, the level of Bmp15 was reduced in the control oocytes but was unchanged in the calcein-AM treated oocytes. However, no effect of calcein-AM treatment on Bmp15 levels in rat oocytes was observed. Cx37 mRNA levels in rat oocytes were significantly affected by both treatment and time. Cx37 mRNA was reduced in the control oocytes after 13 and 25 hours, however this decrease was not observed in the calcein-AM treated oocytes. Significant time and calcein-AM treatment effects were observed for Gdf9 mRNA levels in rat oocytes. Gdf9 mRNA was decreased after 13 and 25 hours in the control group, however this decrease was not observed in the calcein-AM treated group.

For most future gene expression studies, only the 1 and 25 hour incubation times were included. Therefore, the data were reanalysed with the 5 and 13 hour time points excluded. When only the 1 and 25 hour time points were included from the Atr analysis, both treatment $(P<0.05)$ and time effects $(P<0.0001)$ were observed. Expression levels of Atr mRNA were decreased at 25 hours, compared to 1 hour, in both the control and calcein-AM treated oocytes. In addition, Atr mRNA was lower in the calcein-AM treated oocytes compared to the control oocytes at both time points. No treatment, time, or treatment $x$ time interaction effects were observed for Bmp15 levels. Time effects $(P<0.01)$ were noted for $C \times 37$ and Gdf9 levels ( $\mathrm{P}<0.01$ and 0.05 respectively), but no treatment or treatment $\mathrm{x}$ time interaction effects were measured. $C \times 37$ and Gdf9 mRNA levels were decreased over time in the control oocytes and, to a lesser extent, in the calcein-AM treated oocytes. These results are summarised in Table 4-2. 
A

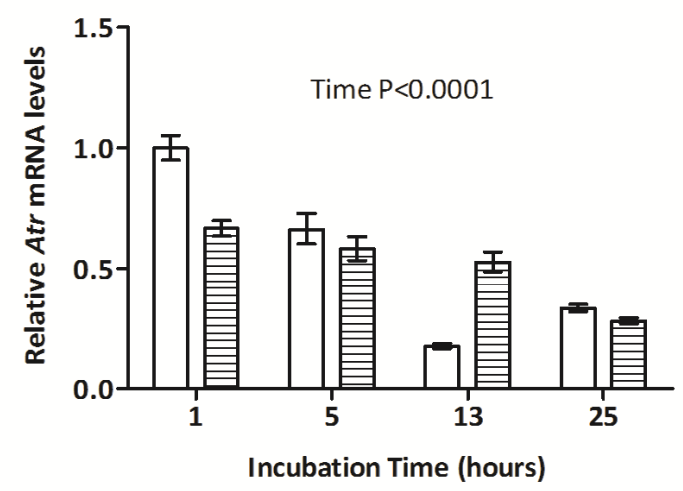

C

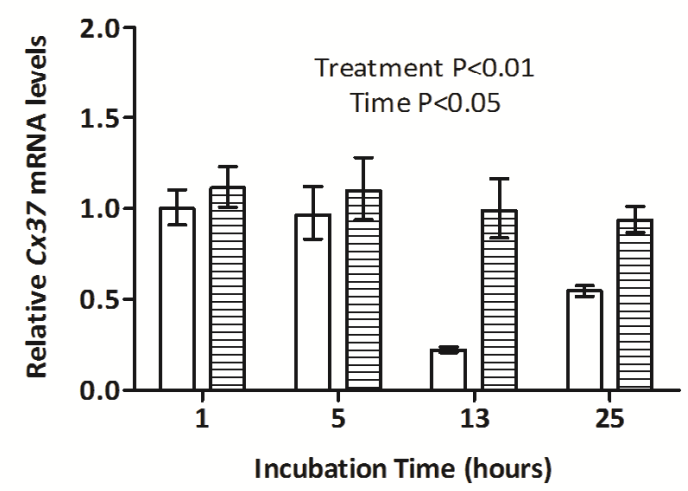

B

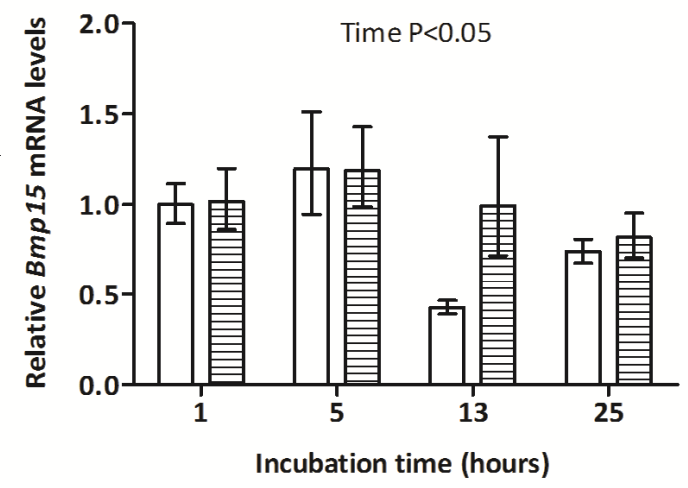

D

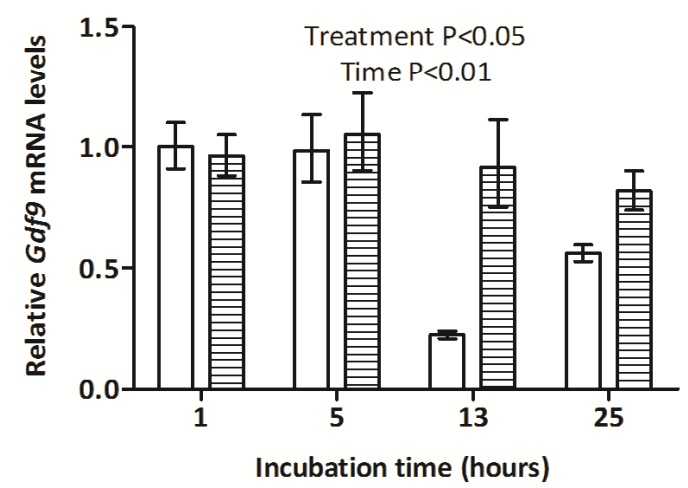

Figure 4-6: Effect of calcein-AM with time on mRNA levels of Atr (A), Bmp15 (B), Cx37 (C), Gdf9 (D) in rat oocytes. The incubation time indicates the pre-calcein incubation + the 1 hour post-calcein incubation. The control is represented by the unfilled bars $(\square)$, while the calcein-AM treated group is represented by the hatched bars (可). Values are shown as means \pm SEM analysed from linearised gene expression values normalised against the mean of the control group at 1 hour, which has a value of 1 . The data represent 7-16 readings from individual oocytes in each group generated from 3 replicate experiments. $B, C$, and $D$ were transformed $(1 / \mathrm{y}+1)$ and $\mathrm{A}$ was not transformed prior to analysis by a two-way ANOVA.

Table 4-2: Summary of calcein-AM treatment, time, and interaction effects on mRNA levels of candidate genes in rat oocytes. Genes are ordered from most abundant to least abundant.

\begin{tabular}{|c|c|c|c|c|c|c|c|}
\hline \multirow[t]{2}{*}{ Gene } & \multirow{2}{*}{$\begin{array}{c}\text { Abundance } \\
\text { relative to } \\
\text { Rpl19 }\end{array}$} & \multicolumn{3}{|c|}{ All time points } & \multicolumn{3}{|c|}{1 and 25 hours only } \\
\hline & & $\begin{array}{c}\text { Treatment } \\
\text { effect }\end{array}$ & Time effect & $\begin{array}{c}\text { Treatment } \\
\text { x Time } \\
\text { effect }\end{array}$ & $\begin{array}{c}\text { Treatment } \\
\text { effect }\end{array}$ & Time effect & $\begin{array}{c}\text { Treatment } \\
\text { x Time } \\
\text { effect }\end{array}$ \\
\hline Gdf9 & $7.93 \pm 0.36$ & $P<0.05$ & $P<0.01$ & - & - & $P<0.05$ & - \\
\hline$C \times 37$ & $7.80 \pm 0.61$ & $P<0.01$ & $P<0.05$ & - & - & $P<0.01$ & - \\
\hline Bmp15 & $1.62 \pm 0.08$ & - & $P<0.05$ & - & - & - & - \\
\hline Atr & $0.35 \pm 0.02$ & - & $P<0.05$ & - & $P<0.05$ & $P<0.0001$ & - \\
\hline
\end{tabular}


The effects of calcein-AM treatment on the expression levels of candidate genes of interest for the CC (i.e. Atr, Cycs, Cx43, Gfpt1, and Pfkp) are shown in Figure 4-7. Significant treatment $\mathrm{x}$ time interaction and time effects were observed for Atr mRNA levels in rat CC. The mean Atr mRNA levels were more variable in the calcein-AM group as evidenced from the SEM. In the control group, Atr mRNA in CC declined progressively after the 5 hour incubation whereas this decline wasn't evident in the calcein-AM treated CC until the 25 hour time point. A significant effect of time was observed for $C_{X} 43$ levels in rat CC. $C_{x} 43$ mRNA levels were markedly reduced from 5 hours onwards in both the control and calceinAM treated CC. No effect of calcein-AM treatment was observed for Cx43 expression. Significant treatment, time and treatment $x$ time interaction effects were observed for Cycs levels in rat CC. Cycs mRNA levels had decreased from 5 hours onwards in the control CC, however levels in the calcein-AM treated CC did not decrease until 25 hours. The mean CycS mRNA levels were consistently higher in the calcein-AM treated CC, compared to the control CC, at 5, 13, and 25 hours. Significant treatment, time and treatment $x$ time interaction effects were observed for Gfpt1 mRNA levels in rat CC. The mean Gfpt1 levels had declined from 5 hours and remained low thereafter in the control CC, However, in the calcein-AM treated CC, the mean mRNA levels appeared to be different with a decrease noted at 5 hours but not at 13 or 25 hours. A significant effect of time was observed for Pfkp mRNA levels in rat CC. Pfkp mRNA was markedly decreased from 5 hours onwards in both the control and the calcein-AM treated CC. No effect of calcein-AM treatment on Pfkp levels was observed.

A further re-evaluation of these data for CC gene expression was undertaken using only the 1 and 25 hour data sets as it seemed that the effects of calcein-AM had their major interactive effects at the 5 and 13 hour time points. When this restricted data set was analysed for Atr, a significant effect of time $(\mathrm{P}<0.05)$ but no treatment or time $\mathrm{x}$ treatment interaction effects were observed. For $C_{x} 43, C y c s$, and Pfkp, significant effects of time (all genes, $\mathrm{P}<0.0001)$ but no treatment or time $\mathrm{x}$ treatment interaction effects were found. However, for Gfpt1, there were significant effects of time $(P<0.0001)$, treatment $(P<0.05)$ and a treatment $x$ time interaction $(P<0.01)$. These results are summarised in Table 4-3. 
A

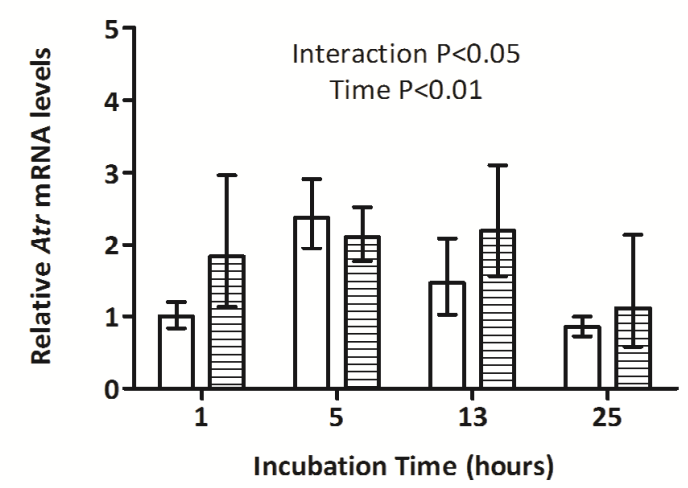

C

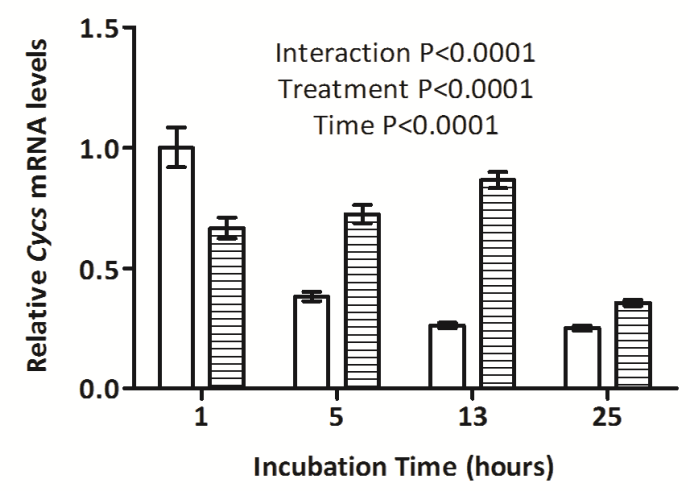

E

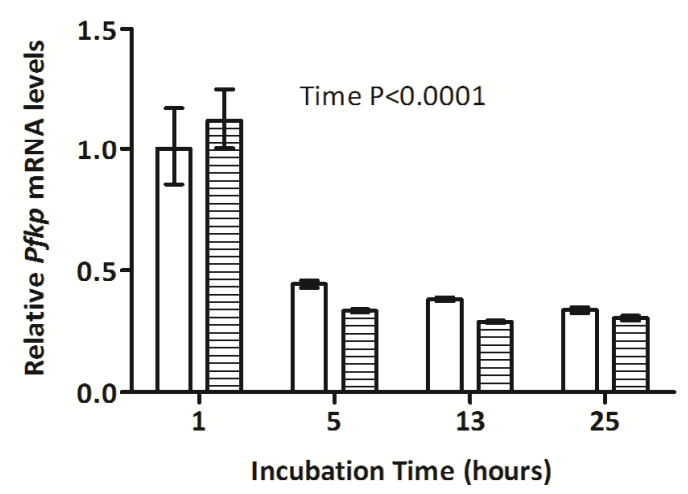

B

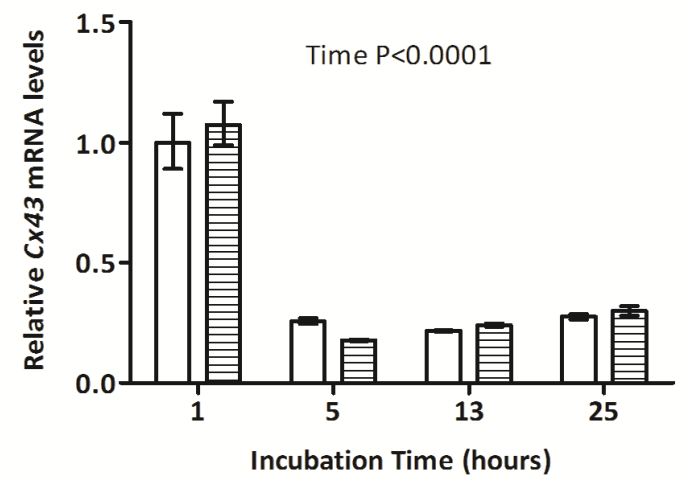

$\mathrm{D}$

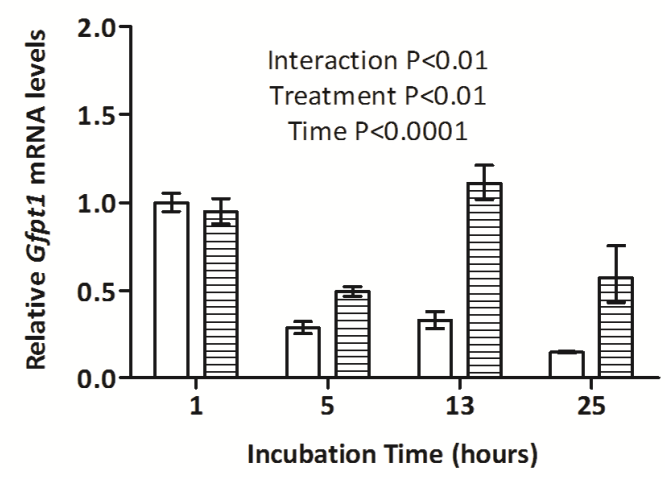

Figure 4-7: Effect of calcein-AM with time on mRNA levels of Atr (A), Cx43 (B), Cycs (C), Gfpt1 (D), and Pfkp (E) in rat CC. The incubation time indicates the pre-calcein incubation + the 1 hour post-calcein incubation. The control is represented by the unfilled bars $(\square)$, while the calcein-AM treated group is represented by the hatched bars (可). Values are shown as means \pm SEM analysed from linearised gene expression values normalised against the mean of the control group at 1 hour, which has a value of 1 . The data represent 9-16 readings from individual oocytes in each group generated from 3 replicate experiments. $C$ was transformed (square $\operatorname{root}(\mathrm{y}+0.5)$ ) and $\mathrm{E}$ was transformed (natural $\log$ ) prior to analysis by a two-way ANOVA. A, B and D were ranked and a two-way ANOVA was performed on the ranked data. 
Table 4-3: Summary of calcein-AM treatment, time, and interaction effects on mRNA levels of candidate genes in rat CC. Genes are ordered from most abundant to least abundant.

\begin{tabular}{cccccccc}
\hline Gene & $\begin{array}{c}\text { Abundance } \\
\text { relative to } \\
\text { Rp/19 }\end{array}$ & \multicolumn{3}{c}{$\begin{array}{c}\text { All time points } \\
\text { effect }\end{array}$} & $\begin{array}{c}\text { Treatment Time effect } \\
\text { Treatment } \\
\text { x Time } \\
\text { effect }\end{array}$ & $\begin{array}{c}\text { Treatment Time effect } \\
\text { effect }\end{array}$ & $\begin{array}{c}\text { Treatment } \\
\text { x Time } \\
\text { effect }\end{array}$ \\
\hline Cx43 & $2.231 \pm 0.31$ & - & $\mathrm{P}<0.0001$ & - & - & $\mathrm{P}<0.0001$ & - \\
Pfkp & $0.178 \pm 0.02$ & - & $\mathrm{P}<0.0001$ & - & - & $\mathrm{P}<0.0001$ & - \\
Cycs & $0.068 \pm 0.004$ & $\mathrm{P}<0.0001$ & $\mathrm{P}<0.0001$ & $\mathrm{P}<0.0001$ & - & $\mathrm{P}<0.0001$ & - \\
Gfpt1 & $0.056 \pm 0.01$ & $\mathrm{P}<0.01$ & $\mathrm{P}<0.0001$ & $\mathrm{P}<0.01$ & $\mathrm{P}<0.05$ & $\mathrm{P}<0.0001$ & $\mathrm{P}<0.01$ \\
Atr & $0.047 \pm 0.005$ & - & $\mathrm{P}<0.01$ & $\mathrm{P}<0.05$ & - & $\mathrm{P}<0.05$ & - \\
\hline
\end{tabular}

\subsection{Discussion}

Fluorescence was detected on the green channel in the sample of diluted calcein-AM solution, indicating that the thermal cycling caused the removal of the acetoxymethyl group resulting in activation of fluorescence. However, fluorescence was not detected in the lysate of oocytes from $\mathrm{COC}$ that had been treated with calcein-AM suggesting that residual calcein contained within the oocytes is at undetectable concentrations. Similarly, no fluorescence was detected in cDNA from oocytes that had been treated with calcein-AM. These results demonstrate that residual calcein fluorescence in oocytes is unlikely to add to or interfere with the fluorescence emitted from any of the TaqMan probes during qPCR reactions.

In this study, the incubation times of $1,5,13$, and 25 hours differed in the lengths of time COC are incubated before exposure to calcein-AM (i.e. $0,4,12$, or 24 hours respectively). However, thereafter the time that COC are exposed to calcein-AM (i.e. 10 minutes) and the subsequent calcein-AM free interval (i.e. 1 hour) were identical for all samples. While it might be expected that exposure to calcein-AM would affect gene expression in a similar manner across all time points, it is also possible that since the functions of individual COC after isolation are so variable, they are much more sensitive to calcein-AM effects over the first 13 hours of incubation. As a consequence of this variability, most future gene expression studies after calcein-AM treatment were limited to the 1 and 25 hour time points. 
The reference gene (Rp/19) used in this study is a component of the ribosome 60S subunit, and is a commonly used reference gene in CC and GC (Dragovic et al., 2006; Feuerstein et al., 2007; Su et al., 2008). Selection of a stable reference gene that is expressed at similar levels to the candidate genes and is not affected by experimental conditions is essential. Two other reference genes commonly used for COC are Gadph and 18s RNA. These were not considered for the current study as Gapdh levels are affected by oestrogens (Diel et al., 2000), and therefore would not be suitable when testing compounds with oestrogenic activity such as BPA, while 18s RNA is very highly expressed and therefore would not provide a good comparison for the candidate genes selected for this study. This current study revealed that Rp/19 is strongly correlated to CC number $(\mathrm{P}<0.0001)$. All of the CC-derived candidate genes were corrected for Rpl19 to remove potentially large amounts of variability in CC-derived candidate gene expression as a result of differences in CC number. The exposure of $\mathrm{COC}$ to the fluorescent dye calcein-AM used in the GJ assay did not directly affect expression of Rp/19 in rat CC, when all incubation times were taken into account. While, Rp/19 levels declined with time this was likely due to reduced CC number as COC diameter was significantly reduced at 13 and 25 hours, compared to at 1 hour $(P<0.01$; Kruskal-Wallis test). Therefore, $R p / 19$ is a suitable reference gene for rat CC.

Interestingly, Rpl19 gene expression levels were not correlated to oocyte size. None of the oocyte genes were corrected for Rp/19, but were instead corrected for a calibrator control sample. Thus for the oocyte genes, mRNA levels were calculated as expression levels in each single DO. Although oocyte-derived candidate genes were not corrected for a reference gene, Rpl19 was measured in all future DO samples, as levels below the detectable level suggested that the RNA extraction was unsuccessful. DO samples that had an Rp/19 $C_{\mathrm{T}}$ value $>30$ were excluded for this reason.

The exposure of COC to the fluorescent dye calcein-AM used in the GJ assay did not directly affect expression of Rp/19 in rat CC or DO, when all incubation times were taken into account. Thus, we conclude that treatment with calcein-AM does not inhibit or interfere with the RNA extraction and cDNA synthesis procedures.

When analysing only the 1 and 25 hour time points, there was no effect of calcein-AM treatment on levels of Rp/19 mRNA in the oocyte. However, a treatment $\mathrm{x}$ time interaction 
was noted for Rpl19 levels in CC. These levels were increased in the calcein-AM treated CC at 1 hour, but were at similar levels to the control CC at 25 hours. It is unlikely that this difference in Rpl19 was a direct result of calcein-AM exposure but rather was due to a difference in the number of CC collected. Indeed, when reviewing the pictures that were taken of each individual $\mathrm{COC}$, those that were collected in the calcein-AM treated group at 1 hour had noticeably more CC than those in the untreated group.

When all of the time points were included in the analyses of the effects of calcein-AM on oocyte-derived genes, a treatment effect was observed for two genes ( $C \times 37$ and Gdf9). Interestingly, Cx37 and Gdf9 were the most abundant genes measured in the oocyte. When only the 1 and 25 hour time points were included in the analysis, no treatment effects on Cx37 and Gdf9 were observed. In fact, including the 1, 5, and 25 hour time points (i.e. excluding the 13 hour time point) also removes the treatment effect. These results indicate that the difference in the levels of these genes between the calcein-AM treated and control groups at 13 hours contribute disproportionately to the treatment effect observed when all the time points were analysed. After 13 hours of incubation, the expression of $C_{x 3} 3$ and Gdf9 may be particularly sensitive to perturbations. In the control oocytes, both $C \times 37$ and Gdf9 were reduced at 13 hours however, calcein-AM may influence this reduction in gene expression.

When only the 1 and 25 hour time points were included in the analyses of the effects of calcein-AM on oocyte-derived genes, a treatment effect was observed for Atr. The gene Atr was the least abundant of all the oocyte genes measured in this study. Oocyte-derived Atr was reduced in the calcein-AM treated group compared to the control group. The effect of calcein-AM treatment on oocyte Atr levels was the same at both time points. In addition, the expression levels of Atr were closely related to the levels of Rp/19, especially at 1 hour, indicating this effect may have been due to differences in RNA input. The effect of calceinAM on Atr mRNA levels in the oocyte may be related to the function of ATR as a DNA damage sensor and regulator of meiosis. The down-regulation of Atr levels in calcein-AM treated oocytes indicate that calcein-AM may protect against DNA damage, resulting in a reduced requirement for $A T R$, or that calcein-AM prevents the oocyte from responding to DNA damage by reducing Atr expression. Either way, this effect of calcein-AM may mask any potential effects of lifestyle factors on Atr levels and the DNA damage pathway. It is possible 
that calcein-AM exposure simply reduces the baseline level of Atr in oocytes, however any interpretations of Atr levels will be treated with caution in future experiments.

When all of the time points were included in the analyses of the effects of calcein-AM on CCderived genes, a treatment effect was observed for two CC-derived genes (Cycs and Gfpt1) while a treatment $x$ time interaction was noted for three of the genes (Atr, Cycs and Gfpt1). The genes Atr, Cycs and Gfpt1 were the least abundant of all the genes measured in the CC. When only the 1 and 25 hour time points were analysed, the treatment effect was not observed for Cycs and no treatment $\mathrm{x}$ time interaction effects were noted for Atr or Cycs. The Atr mRNA levels reduced over time, and this reduction began after 13 hours. However, calcein-AM exposure appeared to delay the reduction in Atr expression, resulting in a large difference in levels between the calcein-AM treated and control groups at 13 hours. The Cycs and Gfpt1 levels were also reduced over time in the control CC from 5 hours onwards. However, calcein-AM treatment appeared to delay the reduction in Cycs levels until 25 hours. This resulted in large differences in the levels of Cycs mRNA between the calcein-AM treated and control CC at 5 and 13 hours. The Gfpt1 levels in the calcein-AM treated CC were similar to the control at 5 hours, but were significantly upregulated at 13 and 25 hours.

When only the 1 and 25 hour time points were included in the analyses of calcein-AM on CC gene expression, treatment and treatment $x$ time interaction effects were only observed for one gene (Gfpt1). CC-derived Gfpt1 was increased in the calcein-AM treated group at 25 hours, but not at 1 hour. This treatment $x$ time interaction effect is of particular concern, as it indicates that the effect of calcein-AM on Gfpt1 expression is inconsistent over time. These results suggest that flux through the hexosamine biosynthetic pathway is upregulated at 25 hours in calcein-AM treated CC. Thus, the interpretation of CC-derived Gfpt1 expression levels over time in $\mathrm{COC}$ subjected to the GJ assay will be treated with caution.

The ability to compare directly the degree of the GJ communication (as determined by the GJ assay) and the expression levels of genes thought to be involved in maintenance of oocyte health is a very powerful tool. This study concludes that despite a few small and transitory differences in expression levels of a couple of genes following the exposure of $\mathrm{COC}$ to calcein-AM, it would be acceptable to measure gene expression levels by multiplex qPCR in $\mathrm{CC}$ and oocytes from $\mathrm{COC}$ that were subjected to the GJ assay. However, when gene 
expression is measured at $1,5,13$, and 25 hours, caution must be taken when interpreting the changes in mRNA levels of oocyte-derived Cx37 and Gdf9 and CC-derived Atr, Cycs, and Gfpt1 due to the effects of calcein-AM exposure on expression of these genes. In addition, when gene expression is measured at 1 and 25 hours, the levels of oocyte-derived Atr and CC-derived Gfpt1 must also be interpreted carefully. 


\section{EFFECT OF TIME IN CULTURE ON COC HEALTH}

\subsection{Introduction}

Removal of the COC from the follicle, and subsequent in vitro culture can have a significant impact on $\mathrm{COC}$ health. As described previously, aspiration of the $\mathrm{COC}$ from the follicular environment results in the progression of meiosis within the oocyte (Wert and Larsen, 1989). Additionally, the communication network comprised of GJs appears to undergo dynamic changes following the removal of $\mathrm{COC}$ from the follicle. For example, GJ communication in bovine COC decreased progressively over 21 hours in vitro (Thomas et al., 2004). However, in porcine COC, GJ communication increased over the first 18 hours of culture, before declining at 24 hours and then remaining unchanged for 24 hours thereafter (Sasseville et al., 2009a). Thus, species differences exist in the impact of removing the $\mathrm{COC}$ from its follicular environment.

Alterations in GJ communication within the $\mathrm{COC}$ would be expected to be associated with molecular and metabolic changes in both the CC and oocyte. Expression of Gdf9 and Bmp15 mRNA transcripts were reduced in rat DO that had been co-cultured with GC for 24 hours (Lin et al., 2012). Utilization of radioactively labelled glucose $\left(\left[5-{ }^{3} \mathrm{H}\right]\right.$ glucose) was observed to increase during IVM of bovine COC over 24 hours (Steeves and Gardner, 1999). Moreover, bovine $\mathrm{CC}$ that were isolated from $\mathrm{COC}$ that underwent IVM had a two-fold increase in cell death, along with increased expression levels of genes involved in apoptosis, compared with $\mathrm{COC}$ that were matured in vivo. Furthermore, $\mathrm{COC}$ that underwent IVM compared with those matured in vivo had augmented activation of the TGF $\beta$ and MAPK signalling pathways and increased mRNA and protein levels of hyaluronan synthase 2 , which is responsible for the synthesis of hyaluronic acid for CC expansion (Salhab et al., 2012). Therefore, while there is some evidence that the removal of the COC from the follicle and its subsequent incubation in vitro can have marked changes in cell-to-cell communication, gene expression levels and metabolic activity in $\mathrm{COC}$ of some species, there is little information regarding these changes in the rat.

The aim of this chapter was to determine the impact of incubation time on the health of rat COC in this study. Specifically, the changes in GJ communication, candidate gene expression (oocyte-derived Atr, Bmp15, Cx37, and Gdf9 and CC-derived Atr, Cx43, Cycs, Gfpt1, and Pfkp) 
and protein expression levels (oocyte-derived GDF9 and CC-derived CX43) in COC that had been incubated for varying amounts of time were investigated.

\subsection{Methods}

\subsubsection{Effect of time on GJ communication}

Ovaries were extracted into dissection media supplemented with $50 \mu \mathrm{M}$ dipyridamole and $50 \mu \mathrm{M}$ rolipram, and the COC were recovered and transferred into a 48-well plate as described previously (section 2.1.1 and 2.1.2). The COC were washed twice in wash media containing $50 \mu \mathrm{M}$ dipyridamole and $50 \mu \mathrm{M}$ rolipram. The COC were then incubated for 0,4 , 12 , or 24 hours in incubation media containing $50 \mu \mathrm{M}$ dipyridamole and $50 \mu \mathrm{M}$ rolipram. The COC were exposed to $2 \mu \mathrm{M}$ calcein-AM for 10 minutes before being incubated for a further hour in fresh incubation media (calcein-AM free) containing $50 \mu \mathrm{M}$ dipyridamole and $50 \mu \mathrm{M}$ rolipram. The COC were transferred into incubation media free of PDE inhibitors where the oocyte was denuded and then fluorescence was measured. The oocytes and separate CC masses were then stored at $-80{ }^{\circ} \mathrm{C}$ until further processing for RNA extraction. The oocyte fluorescence for each individual sample was corrected for the geometric mean of that at 1 hour. The relative fluorescent levels were analysed by a Kruskal-Wallis test, and where a significant difference was observed, a Dunn's multiple comparison post hoc test was performed.

\subsubsection{Effect of time on mRNA levels}

Total RNA was extracted from the DO and CC samples from the GJ bioassay and synthesised into cDNA as previously described (Section 2.3.1). Three quadriplex qPCR reactions were used to measure expression of candidate gene in CC and/or DO. The candidate genes were as follows: CC expression of $C_{x} 43$, Gfpt1 and Pfkp; oocyte expression of Bmp15, Cx37 and Gdf9; and both oocyte and CC expression of Atr, Cycs and Glut1. The reference gene Rp/19 was included in each of qPCR multiplexes. The expression of each gene in each sample was measured in duplicate. Relative levels of mRNA were calculated as described previously (Section 2.3.5). Expression levels of CC genes were corrected for Rp/19, enabling candidate gene expression to be standardized relative to CC number. Expression levels of oocyte genes were corrected for an internal calibrator control, giving total candidate gene expression per 
oocyte. These data were analysed by a Kruskal-Wallis test. Where significant differences were measured, a Dunn's multiple comparison post-hoc test was performed.

\subsubsection{Effect of time on protein expression}

Ovaries were extracted into dissection media supplemented with $50 \mu \mathrm{M}$ dipyridamole and $50 \mu \mathrm{M}$ rolipram, and the $\mathrm{COC}$ were recovered and transferred into a 48-well plate as described previously. The $\mathrm{COC}$ were transferred into incubation media containing $50 \mu \mathrm{M}$ dipyridamole and $50 \mu \mathrm{M}$ rolipram and incubated for $1,5,13$ or 25 hours. These incubation times were chosen to reflect the total incubation period during the GJ assay (i.e. 4 hours incubation plus 1 hour post-calcein incubation). The COC were washed twice in PBS and were collected into a microcentrifuge tube (100 COC/tube). The COC were pelleted by centrifugation and were resuspended in $15 \mu \mathrm{L}$ PBS containing proteinase inhibitors. A total of 200 COC were collected per group, comprising of pooled samples from two collection days.

A $10 \mu \mathrm{L}$ aliquot (the equivalent of $\sim 67 \mathrm{COC}$ ) of each pool of COC was taken and analysed for protein concentration using the BCA assay as previously described (Section 2.4.1). Briefly, $\mathrm{BCA}$ reagent was added to each sample in a 96-well plate, the plate was incubated at $60{ }^{\circ} \mathrm{C}$ for 2 hours, and the absorbance at $490 \mathrm{~nm}$ was measured. The total protein content was calculated by comparing against the $490 \mathrm{~nm}$ absorbance values of a bovine serum albumin standard curve. The remaining $20 \mu \mathrm{L}$ aliquot (the equivalent of $\sim 133 \mathrm{COC}$ ) was analysed for the expression of CX43 and GDF9 proteins using Western blotting as described in Section 2.4. To determine band specificity, the blot was also incubated with either the CX43 or GDF9 specific antibodies pre-absorbed with the appropriate control peptide or recombinant protein.

Band densities were analysed using the ImageJ software. For the CX43 blots, the density of the $43 \mathrm{kDa}$ band was measured. For the GDF9 blots, the densities of the $49 \mathrm{kDa}$ promature and, when present, the $20 \mathrm{kDa}$ mature bands were measured. When multiple bands were unresolved (e.g. when doublet or triplet bands were present for CX43) the density of all bands was measured. The band densities for CX43 protein were corrected for total protein amount, as determined by the BCA assay. The band densities for GDF9 protein were uncorrected to give total GDF9 protein expressed by a known number of oocytes. 


\subsection{Results}

\subsubsection{Effect of time on GJ communication}

The effect of increasing incubation time on GJ communication in rat $\mathrm{COC}$ is depicted in Figure 5-1. Oocyte fluorescence was increased at 5, 19, or 25 hours, compared to that at 1 hour. The proportion of oocytes with a visible GV progressively and significantly decreased over time, with all oocytes containing a GV at 1 hour, but only $44 \%$ of oocytes containing a GV after 25 hours (Table 5-1).

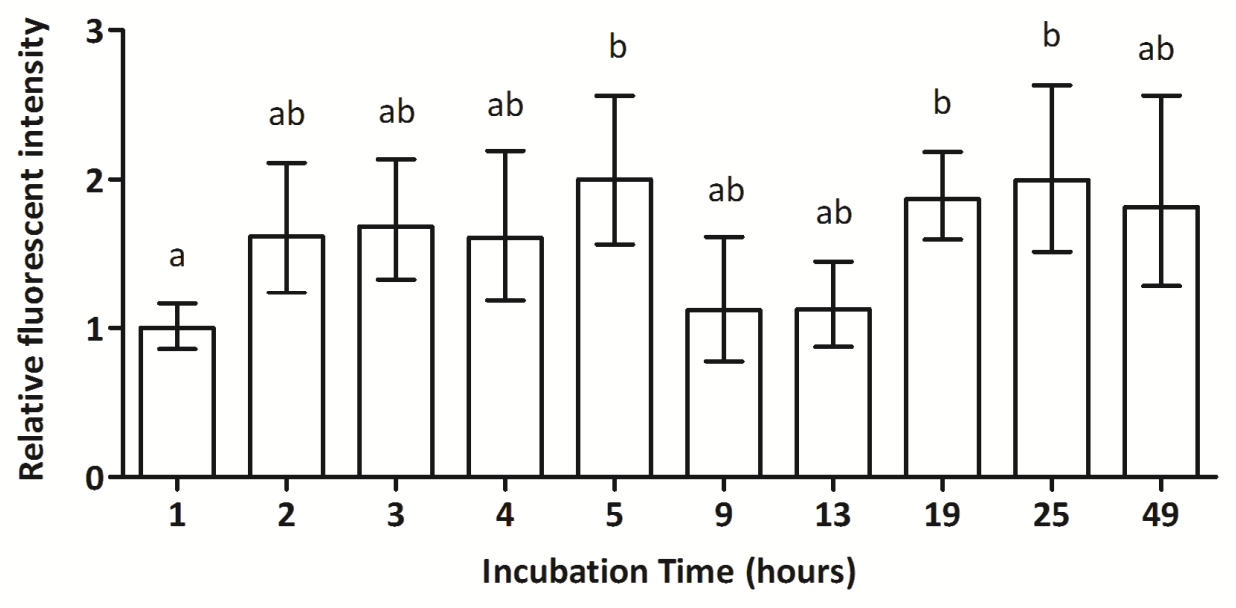

Figure 5-1: Effect of incubation time on fluorescent dye transfer from adjacent CC to oocytes. The incubation time indicates the pre-calcein incubation + the 1 hour post-calcein incubation. Values are shown as geometric means $\pm 95 \%$ confidence intervals of fluorescent ratios normalised against the geometric mean of the 1 control. The data represent 16-17 readings from individual oocytes at each time generated from 3 replicate experiments. The data were analysed using the Kruskal-Wallis test, and where significant differences were observed, a Dunn's multiple comparison post-hoc test was performed. Groups that do not share a letter are significantly different $(P<0.05)$. The mean \pm SEM fluorescent reading at 1 hour was $357 \pm 28$.

Table 5-1: Effect of selected incubation times on the proportion of oocytes with a visible GV. The table shows the proportion (\%) of oocytes with a visible GV, with the actual number of oocytes with a GV in brackets. The data from 3 replicate experiments $(\mathrm{N}=16-17)$ was analysed by a Fisher's exact test. Groups that do not share a letter are significantly different from one another $(P<0.05)$.

\begin{tabular}{cc}
\hline Time (hours) & \% GV oocytes \\
\hline 1 & $100^{\mathrm{a}}(16)$ \\
5 & $88^{\mathrm{ab}}(14)$ \\
13 & $56^{\mathrm{bc}}(9)$ \\
25 & $44^{\mathrm{c}}(7)$ \\
\hline
\end{tabular}




\subsubsection{Effect of time on mRNA levels in rat oocytes and CC}

The effect of increasing incubation time on mRNA levels in cultured rat oocytes is depicted in Figure 5-2. It appeared that levels of Atr mRNA in oocytes declined progressively after 1 hour, and were significantly lower $(P<0.05)$ at 25 hours. Conversely, the expression levels of Bmp15, Cx37, and Gdf9 mRNA in oocytes did not change over time. Oocyte-derived Rp/19 mRNA levels remained unchanged at 5 hours, compared to 1 hour, but appeared to decline at 13 and 25 hours, however this was not significant. Oocyte-derived Cycs and Glut1 mRNA levels were below the detection limit.

The effect of increasing incubation time on mRNA levels in cultured rat CC is depicted in Figure 5-3. The levels of Atr mRNA in CC had increased by 13 hours, compared to 1 hour $(P<0.05)$, in culture but this effect had dissipated by 25 hours. The levels of $C x 43$ mRNA in CC were clearly reduced $(P<0.0001)$ at 5 and 13 hours, compared to that at 1 hour, and remained low after 25 hours. Two samples at 25 hours contained relative Cx43 levels of 1.29 and 1.19 (compared to relative levels of $0.013-0.678$ in the other CC samples) and these samples masked the clear reduction in $C_{x} 43$ mRNA levels at this time point during the statistical analysis. However, these samples were not excluded from the analysis, as the Rp/19 $\mathrm{C}_{\mathrm{T}}$ values were well within the sensitive range. The levels of Cycs mRNA remained constant until 25 hours, at which time they had significantly declined $(P<.05)$. The levels of Gfpt1 mRNA in CC increased between 5 and 13 hours $(P<0.01)$, but this effect was attenuated by 25 hours. The levels of $P f k p$ mRNA were markedly reduced $(P<0.01)$ at all time points, compared to 1 hour. Similar to that observed in the oocyte, Rp/19 mRNA levels declined with time, with a significant difference at 13 and 25 hours compared to 1 hour $(\mathrm{P}<0.05$ for both time points). CC-derived Glut1 mRNA levels were below the detection limit. 
A
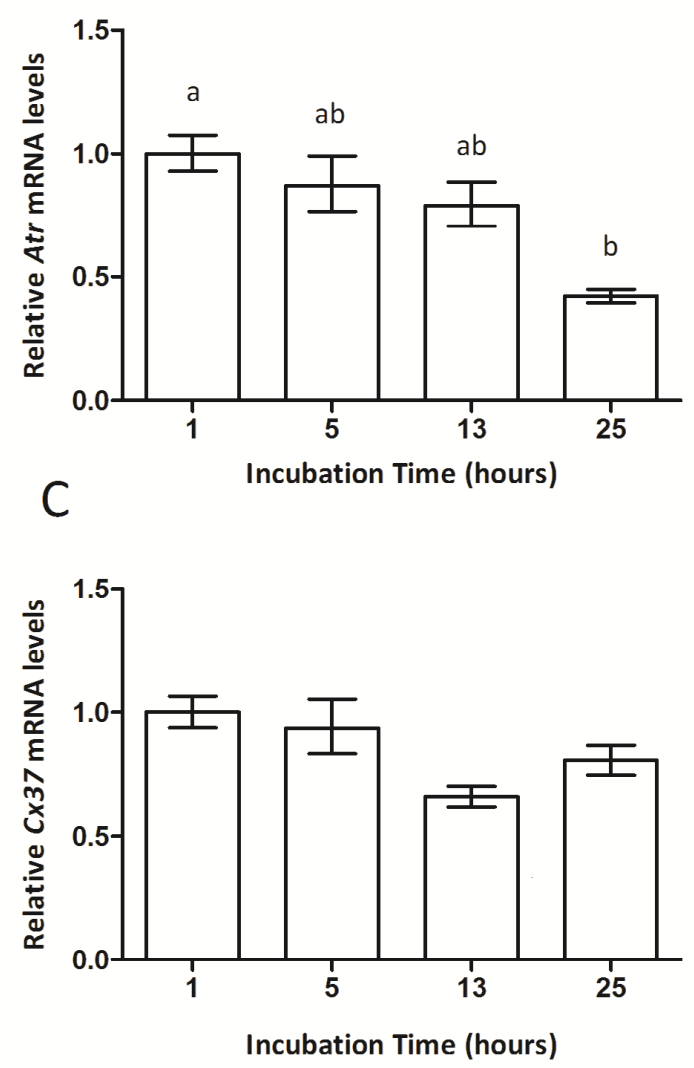

$E$

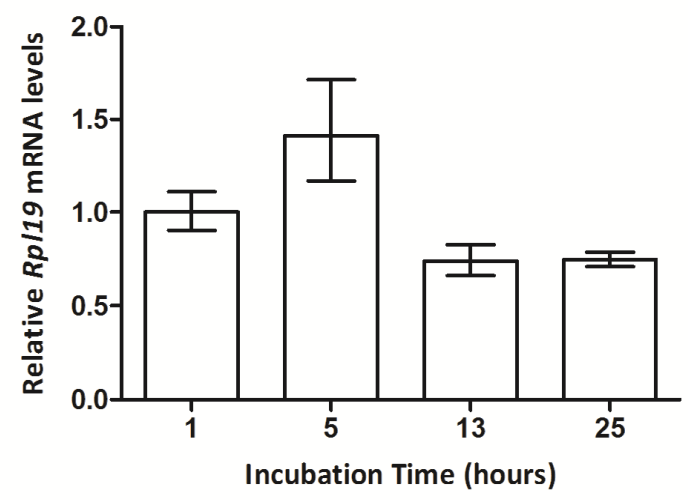

B
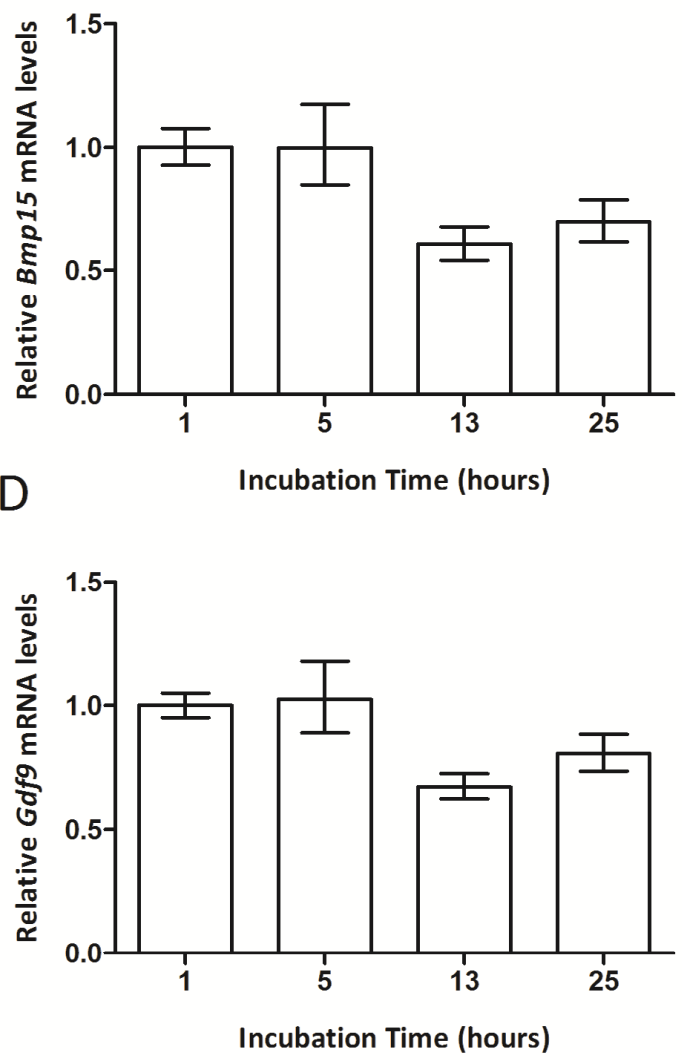

Figure 5-2: Effect of incubation time on mRNA levels of $A \operatorname{tr}(\mathrm{A}), \mathrm{Bmp15}$ (B), Cx37 (C), Gdf9 (D), and Rpl19 (E) in rat oocytes. Values are shown as means \pm upper/lower limit of linearised $C_{T}$ values normalised against the mean of that at 1 hour. The data represent 7-16 readings from individual oocytes in each group generated from 3 replicate experiments. The data were analysed using the Kruskal-Wallis test, and where significant differences were observed, a Dunn's multiple comparison post-hoc test was performed. For Atr levels groups that do not share a letter are significantly different $(\mathrm{P}<0.05)$. No significant effects of time on $B m p 15, C \times 37$, Gdf9, or Rp/19 levels were noted. 
A
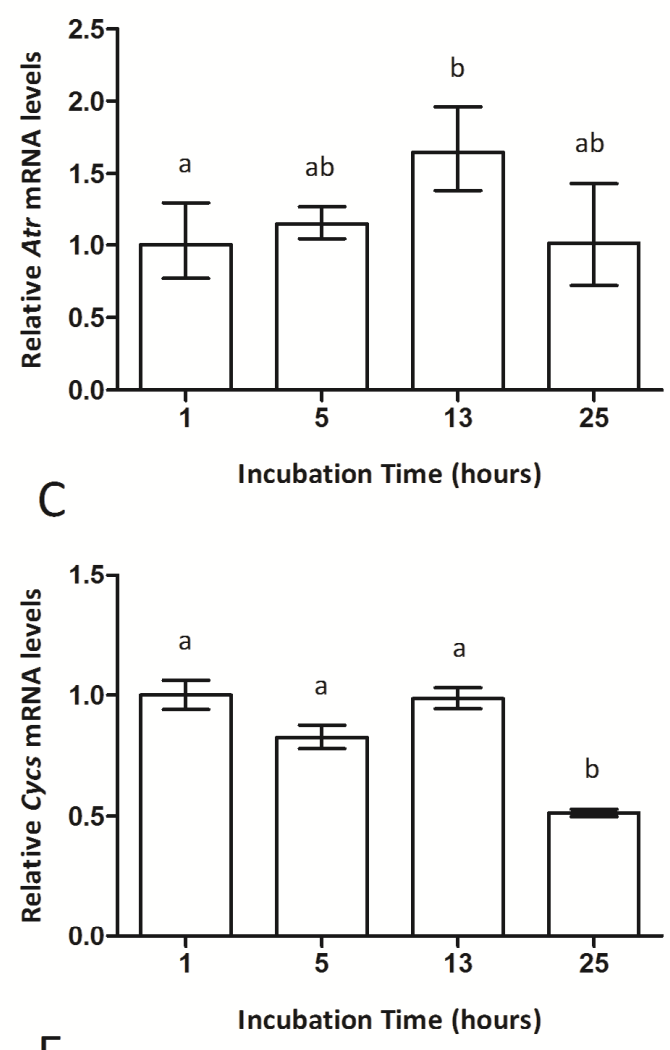

$E$

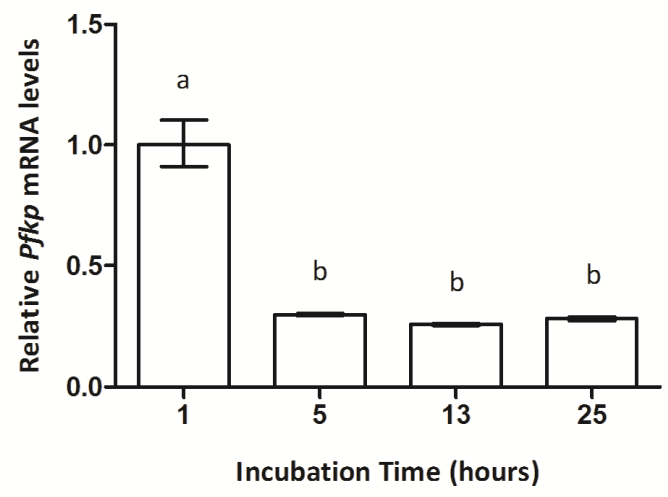

B
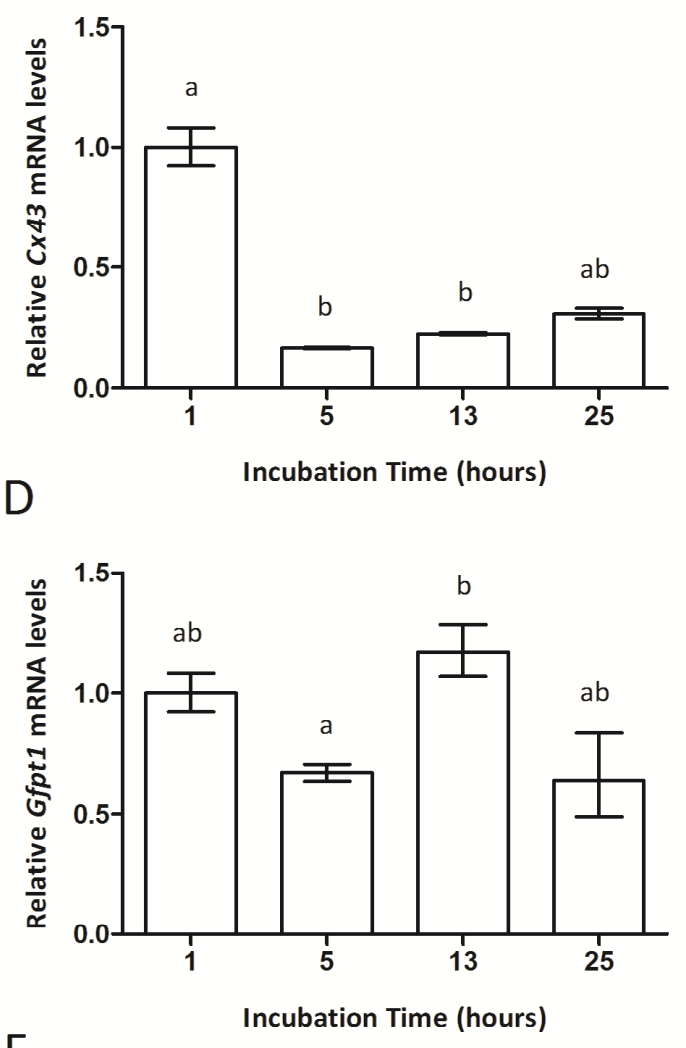

F

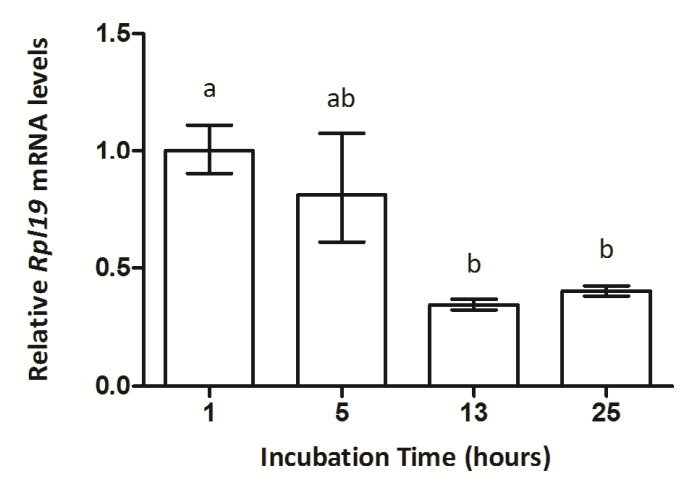

Figure 5-3: Effect of incubation time on mRNA levels of Atr (A), Cx43 (B), Cycs (C), Gfpt1 (D), Pfkp (E), and $R$ pl19 (F) in rat CC masses. Values are shown as means \pm upper/lower limit of linearised $C_{T}$ values normalised against the mean of that at 1 hour. The data represent 7-16 readings from individual CC masses in each group generated from 3 replicate experiments. The data were analysed using the Kruskal-Wallis test, and where significant differences were observed, a Dunn's multiple comparison post-hoc test was performed. In all graphs, groups that do not share a letter are significantly different $(\mathrm{P}<0.05)$. 
The relationship between oocyte fluorescence and expression levels of $C_{x} 43$ and $C_{x 3}$ mRNA, as denoted by $C_{T}$ values, in each individual sample is shown in Figure 5-4. There was a very weak relationship between oocyte fluorescence and $C_{x} 43$ expression levels. Oocytes with higher fluorescence readings tended to be associated with $C C$ with lower $C_{x} 43$ mRNA levels (high $\mathrm{C}_{\mathrm{T}}$ value). No relationship was observed between oocyte fluorescence and expression levels of $C \times 37$ mRNA (denoted by $C_{T}$ values).

A

Cx43

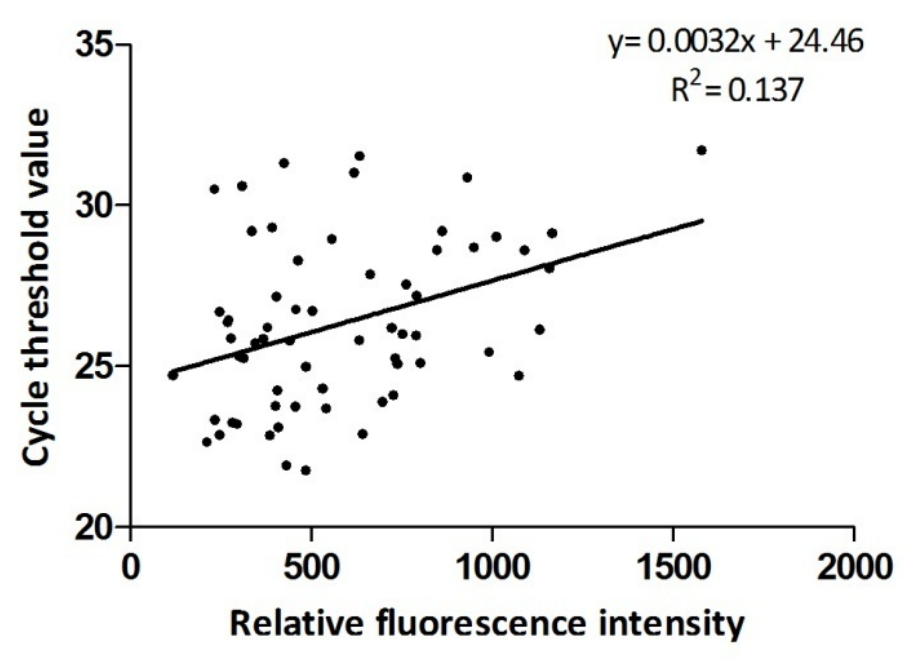

B

Cx37

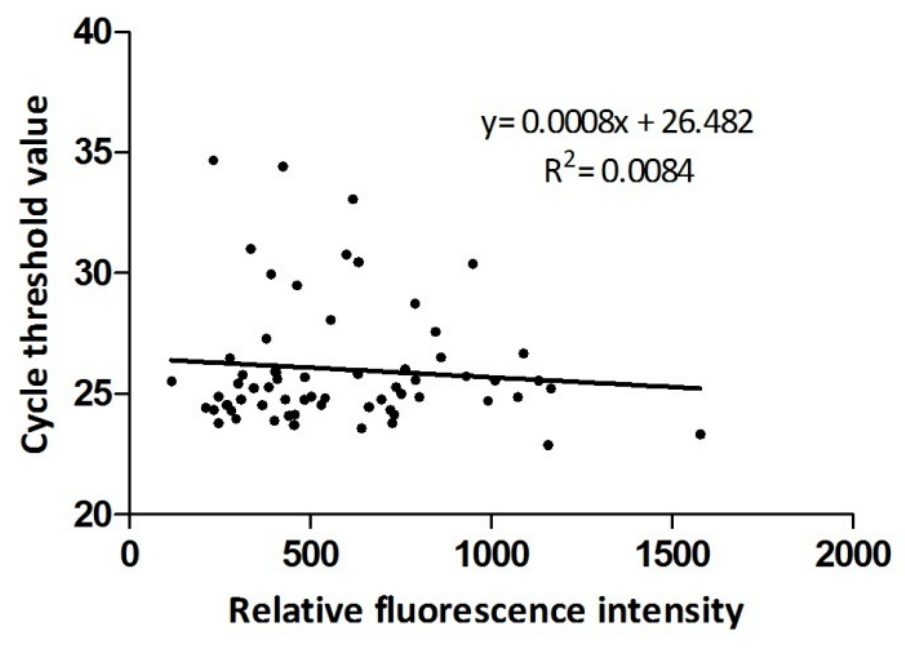

Figure 5-4: Correlation between oocyte fluorescence values and $C_{T}$ values for $C \times 43$ (A) and $C \times 37$ (B). 


\subsubsection{Effect of time on protein levels}

The effect of increasing incubation time on CX43 protein expression in rat COC in culture is depicted in Figure 5-5. Multiple bands were detected in the positive control of both replicates, while no bands were observed in the negative control. A band corresponding to the size of $\mathrm{CX} 43$ protein (i.e. $43 \mathrm{kDa}$ ) was detected in the positive control of the first replicate (Figure 5-5A), along with unidentified bands around 65 and $22 \mathrm{kDa}$ in size. In the second replicate, a $43 \mathrm{kDa}$ band was not detected in the positive control (Figure 5-5B). In contrast to the $\mathrm{COC}$ lysates from the second replicate where only a $43 \mathrm{kDa}$ band was observed, an additional strong unidentified $22 \mathrm{kDa}$ band was observed in the COC lysate from the first replicate. Following stripping of the blot and re-probing with the CX43 antibody that had been pre-absorbed with the control peptide, the bands that had previously been visualised were not apparent (Figure 5-5C).

In comparison with that in $\mathrm{COC}$ after 1 hour in culture, the corrected CX43 protein levels appeared to be slightly reduced at 5 hours (Figure 5-6), markedly increased at 13 hours ( 3 fold higher) and slightly increased at 25 hours (1-2.5 fold higher). There was a large variability in CX43 protein levels between replicates at 25 hours. 
A

\begin{tabular}{|c|c|c|c|c|c|c|}
\hline & 1 & 5 & 13 & 25 & + & - \\
\hline $\begin{array}{l}\text { Uncorrected band } \\
\text { density }\end{array}$ & 1.0 & 0.2 & 1.9 & 1.1 & - & - \\
\hline Total protein content & 4.9 & 4.4 & 3.3 & 5.3 & - & - \\
\hline $\begin{array}{l}\text { Band density/ } \\
\text { protein content }\end{array}$ & 1.0 & 0.2 & 2.9 & 1.0 & - & - \\
\hline
\end{tabular}

\begin{tabular}{llllll}
\hline B \\
\\
27
\end{tabular}

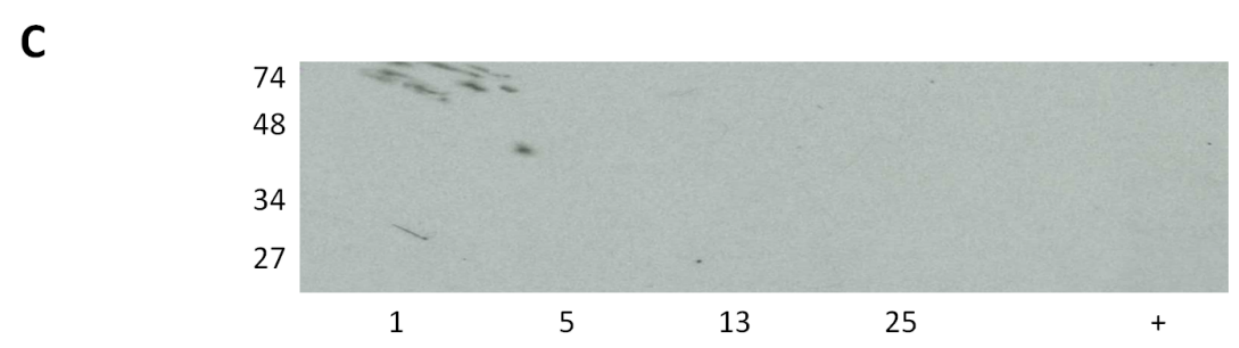

Figure 5-5: The in vitro effect of incubation time on CX43 protein levels in rat COC. Western blots were performed in duplicate (A and $B$ ) to determine the level of CX43 protein in COC lysate. COC were either incubated for $1,5,13$, or 25 hours. Rat ovary lysate (A) or rat aorta lysate (B) were included as positive controls $(+)$ and $E$. coli produced recombinant ovine BMP15 was included as a negative control (-).A control experiment (C) was also run where the membrane (B) was stripped and re-probed with the CX43-specific antibody that had been pre-absorbed with its control peptide. Molecular sizes ( $\mathrm{KDa}$ ) are indicated to the left of each blot. The density of the band of interest (indicated by an arrowhead) was measured and corrected for the control at 1 hour. Total protein $(\mu \mathrm{g})$ in each sample was determined using the BCA assay. The relative band densities were corrected for total protein amount, as calculated by the BCA assay. 


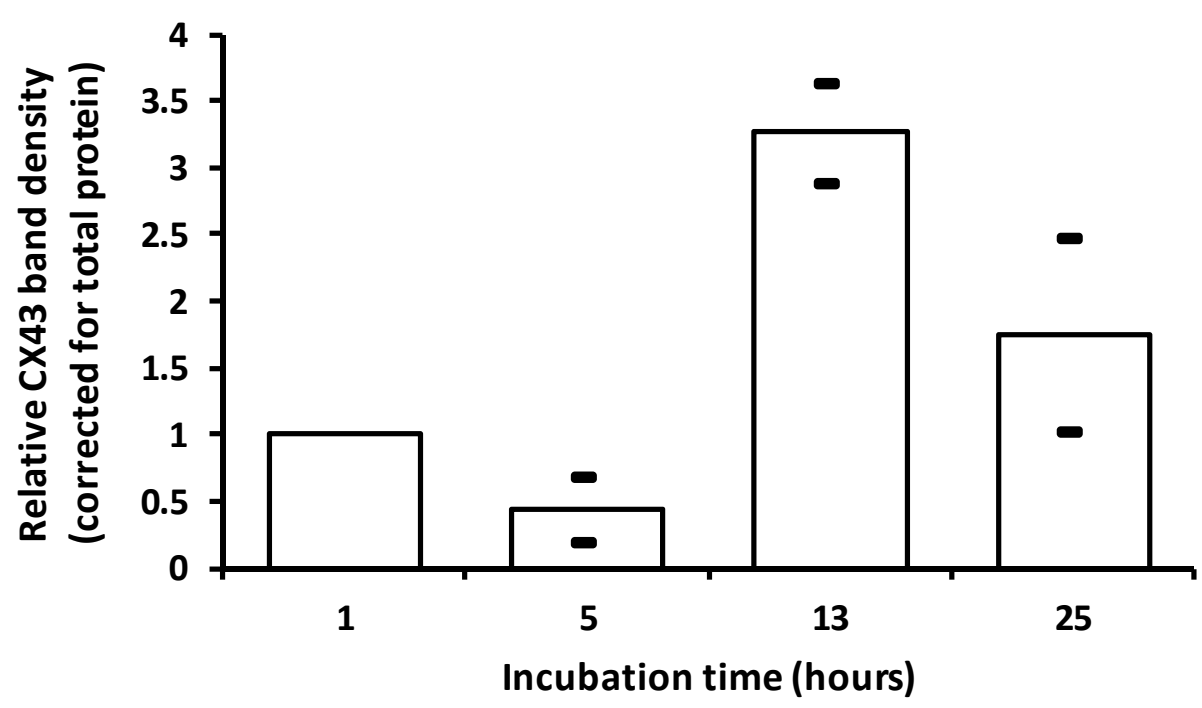

Figure 5-6: Effect of incubation time on relative CX43 levels in rat COC. The bars depict the mean relative CX43 band densities corrected for protein amount at each time point, with each individual band density represented by the dashes.

The effect of increasing the incubation time of rat COC on GDF9 protein levels is depicted in Figure 5-7. Multiple bands between 20 and $114 \mathrm{kDa}$ were detected in the positive control (Figure 5-7A and C) and the COC lysates (Figure 5-7A, B \& C) of both replicates. These included bands of 49 and $20 \mathrm{kDa}$ in size, corresponding to the promature and mature forms of GDF9, respectively. Additional prominent bands of 93, 70, and 40 kDa were also observed. To better visualise the mature $20 \mathrm{kDa}$ band in the COC lysate, the first replicate was subjected to an increased exposure time (Figure 5-7B) and revealed the absence of this band at 5 hours in culture. No bands were observed in the negative control (Figure 5-7A \& C). Following stripping of the blot and re-probing with the GDF9 antibody that had been preabsorbed with recombinant GDF9, the bands that had been visualised were not apparent, with the exception of one band that was markedly reduced in intensity (Figure 5-7D).

Overall, the relative levels of the promature and mature GDF9 proteins did not change over time (Figure 5-8A and B, respectively). There was large variation in the relative levels of both forms of GDF9 at all time points, and especially at 25 hours (i.e. the promature form ranged from 0.8-2 fold; the mature form ranged from 0.5-3.4 fold). 
A

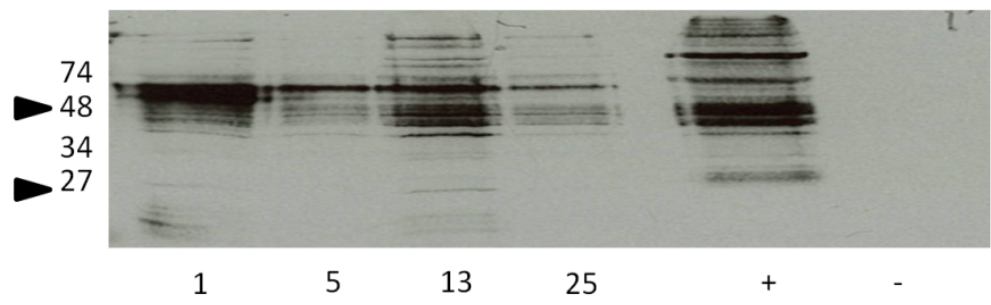

B

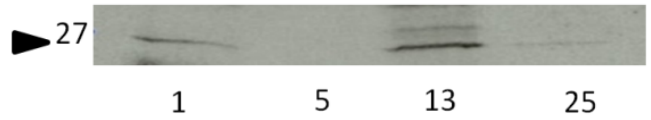

\begin{tabular}{lllllll}
\hline $\begin{array}{l}\text { Uncorrected promature } \\
\text { band density }\end{array}$ & 1.0 & 0.5 & 1.8 & 0.8 & - & - \\
\hline $\begin{array}{l}\text { Uncorrected mature band } \\
\text { density }\end{array}$ & 1.0 & 0.2 & 1.7 & 0.5 & - & - \\
\hline Total protein content & 4.9 & 4.4 & 3.3 & 5.3 & - & - \\
\hline
\end{tabular}

C

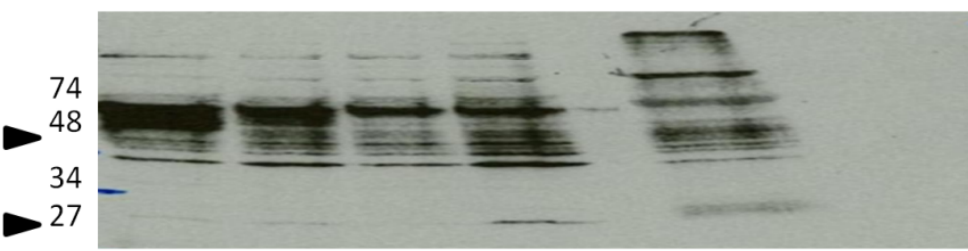

$\begin{array}{llll}1 & 5 & 13 & 25\end{array}$

\begin{tabular}{lcccccc}
\hline $\begin{array}{l}\text { Uncorrected promature } \\
\text { band density }\end{array}$ & 1.0 & 1.4 & 1.0 & 2.0 & - & - \\
\hline $\begin{array}{l}\text { Uncorrected mature } \\
\text { band density }\end{array}$ & 1.0 & 1.1 & 0.9 & 3.4 & - & - \\
\hline Total protein content & 7.0 & 6.4 & 5.2 & 10.2 & - & - \\
\hline
\end{tabular}

D

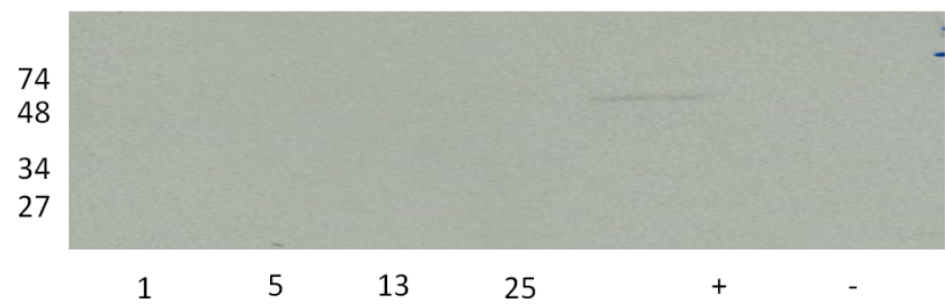

Figure 5-7: The in vitro effects of incubation time on GDF9 protein levels in rat COC. Western blots were performed in duplicate (A and C) to determine the level of GDF9 protein in COC lysate. COC were either incubated for $1,5,13$, or 25 hours. Rat ovary lysate was included as a positive control (+) and E. coli produced recombinant ovine BMP15 was included as a negative control (-).A longer exposure time was performed for blot $(A)$ to better visualise the mature band (B). A control experiment (D) was also run where the membrane (B) was stripped and re-probed with the GDF9-specific antibody that had been pre-absorbed with $E$. coli produced recombinant ovine GDF9. Molecular sizes $(\mathrm{kDa})$ are indicated to the left of each blot. The densities of the bands of interest (indicated by arrowheads) were measured and corrected for the control at 1 hour. Total protein ( $\mu \mathrm{g}$ ) in each sample was determined using the BCA assay. 
A

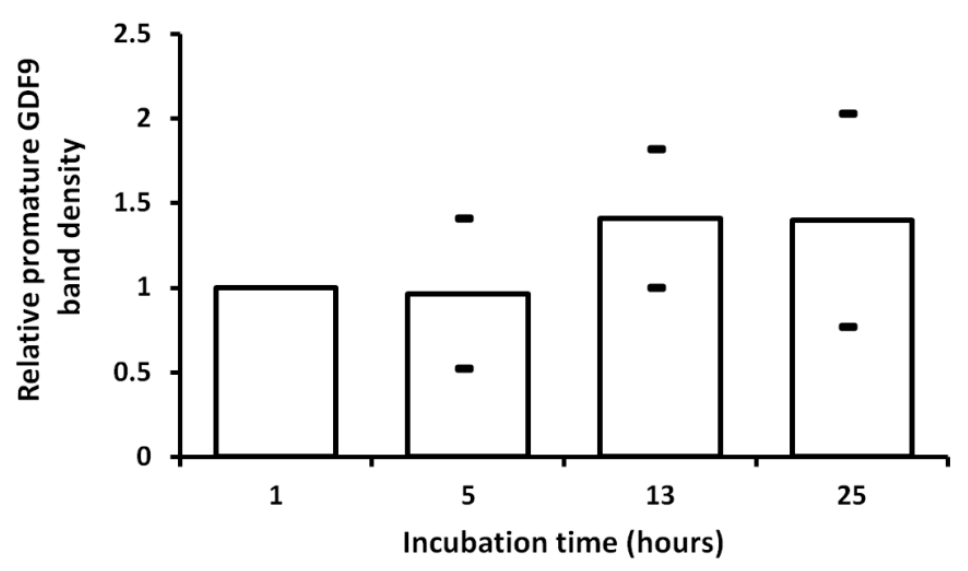

B

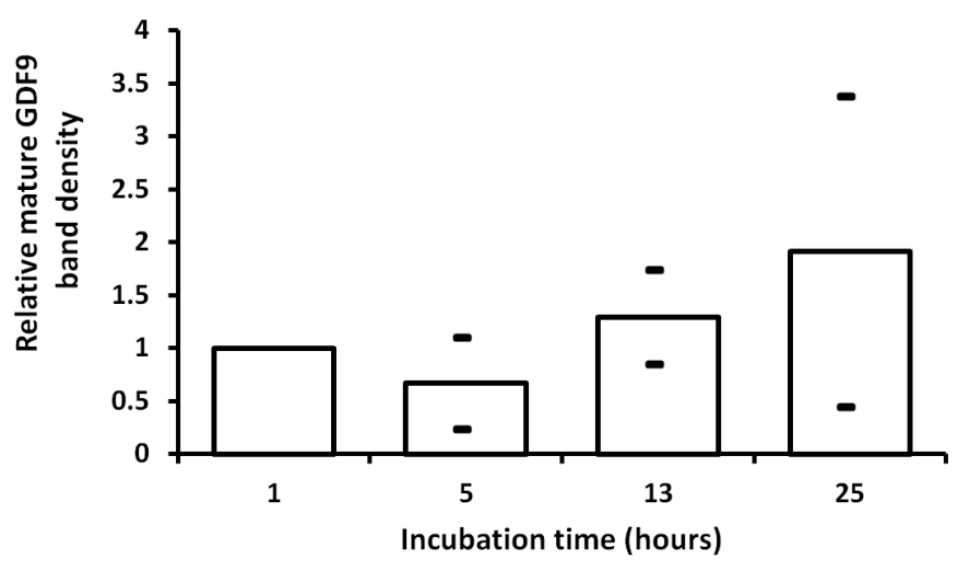

Figure 5-8: Effect of incubation time on promature (A) and mature (B) GDF9 protein levels in rat COC. The bars depict the mean relative GDF9 band densities at each time point, with each individual band density represented by the dashes.

\subsection{Discussion}

The purpose of this chapter was to determine the effects of incubating rat $\mathrm{COC}$ in vitro over an extended time period on GJ communication and the expression of oocyte- and CC-derived candidate genes and proteins. The candidate genes and proteins were selected due to their key roles in COC health. These results demonstrate that a number of cellular processes were altered over time when rat $\mathrm{COC}$ were cultured in vitro.

GJ communication in COC showed a biphasic pattern over time in culture where levels increased from 2-5 hours, declined to low levels between 9-13 hours, and then increased again from 19-25 hours. This pattern of GJ activity is markedly different to that seen in 
cultured porcine and bovine COC (Thomas et al., 2004; Sasseville et al., 2009b). Interestingly in the current study, the pattern of GJ communication over time was not strongly correlated with gene or protein expression levels of the connexins. This indicates that the regulation of GJ communication in rat $\mathrm{COC}$ is more complex than connexin expression levels alone, and may be further regulated by post-translational modifications. In contrast to the current study, GJ communication increased for up to 18 hours in culture in porcine COC, before declining after 24 hours. Moreover, an elegant study by Sasseville et al. (2009b) showed that mechanical rupture of a porcine $\mathrm{COC}$ from the follicle results in a transcriptional-dependent upregulation of CX43 protein after 4 hours in culture, which was concomitant with an increase in GJ communication. However, the following decrease in GJ communication by 22 hours in culture in the Sasseville et al. study was not associated to CX43 protein levels, but to GVBD and compartmentalisation of CX43 into lipid rafts in a gonadotrophin-dependent manner. Taking into account that the timings may be different, it appears this pattern of events does not occur in the rat. The increase in GJ communication in rat $\mathrm{COC}$ observed after 5 hours in culture in the current study was concomitant with a marked decline in both mRNA and proteins levels of $\mathrm{CX} 43$, and no change in $\mathrm{C} \times 37$ mRNA levels. Furthermore, the decrease in GJ communication in rat $\mathrm{COC}$ after 13 hours in culture was associated with a large increase in CX43 protein levels. However it is not known whether this decline in GJ communication was associated with post-translational or cellular localisation changes of CX43 within the COC. One similarity between this study and that of Sasseville et al. was that this decline in GJ communication after 13 hours was associated with an increased proportion of oocytes undergoing GVBD. However in the current study, GJ communication increased thereafter despite the increased proportion of oocytes resuming meiosis.

Interestingly, after 25 hours in our culture system, $\sim 50 \%$ of rat oocytes still contained a visible GV indicating that half of the oocytes were able to maintain meiotic arrest for longer than 25 hours. There was no apparent correlation with time between the level of GJ communication and the proportion of oocytes that had resumed meiosis. It is well established that oocytes require a connection with the CC to maintain meiotic arrest (SelaAbramovich, 2006; Norris et al., 2009; Conti et al., 2012). Whilst the exact molecule supplied by the CC for this purpose is currently being debated, it is clear that the critical end-point is high intra-oocyte cAMP levels. In the present study, PDE inhibitors were added to maintain 
GJ communication and oocyte meiotic arrest over a long incubation period. This was likely to have decreased the need for the transfer of the meiotic maintenance molecule through the GJs, thus confounding any correlation between GJ communication and nuclear maturation that exists in the absence of PDE inhibitors.

The amount of RNA from the individual oocytes, as indicated by Rp/19 mRNA levels, was similar across all time points. Therefore, despite not correcting oocyte-derived candidate gene expression levels against Rp/19 mRNA levels, differences in gene levels are likely to be due to changes in expression, rather than differences in RNA input. The level of Atr mRNA in the oocyte progressively decreased with the lowest levels measured after 25 hours in culture. The reduced level of Atr expression indicates that the DNA damage checkpoint is less active after 25 hours incubation. Both Atr mRNA levels and the proportion of oocytes with a GV decreased over time which is consistent with the role of Atr in the regulation of meiosis. ATR detects DNA damage in the oocyte at the pachytene stage of meiotic prophase (Garcia-Cruz et al., 2009). Activated ATR phosphorylates downstream targets, including CHK1, BRCA1, and NBS1, resulting in $\mathrm{G}_{2} / \mathrm{M}$ arrest (Abraham, 2001). However, the level of Atr mRNA was not significantly associated with the presence or absence of a GV (data not shown). The mRNA levels of Bmp15, $C \times 37$, and Gdf9 remained steady over time. Previously (Chapter 4), it was found that treatment with calcein-AM prevented a time-dependent decrease in $C \times 37$ and Gdf9 levels. Thus, the finding that $C \times 37$ and Gdf9 did not change over time in this study may be attributable to the treatment of all samples with calcein-AM. Interestingly, levels of the promature GDF9 protein also remained steady over time, correlating with Gdf9 mRNA levels. Taken together, these results suggest that the oocyte health is generally unaffected by a 25 hour in vitro incubation period. However, a reduction in the activity of the DNA damage sensing pathway may occur at 13 hours.

The mRNA levels of all of the CC genes tested showed a change over time. The Rp/19 mRNA levels were reduced at the 13 and 25 hour time points, suggesting that fewer CC were attached to the oocyte at later times. Since each candidate gene was corrected for Rp/19, this discrepancy in the number of CC collected at each time point was taken into account. The Atr mRNA levels in CC were increased at 13 hours, compared to 1 hour, indicating that the DNA damage checkpoint is more active at 13 hours, and may correspond to increased DNA damage in those CC that are still attached to the oocyte. In pigs, DNA damage in the 
oocyte as a result of oxidative stress generated by the xanthine oxidoreductase (XOD) enzyme system was reduced when the oocytes were cultured as $\mathrm{COC}$, rather than as DO (Tatemoto et al., 2000). Given the finding that oocyte Atr levels were relatively constant until 13 hours in culture, and then reduced at 25 hours, this supports the hypothesis that the CC protect the oocyte from DNA damage. As discussed previously, Cx43 mRNA levels were reduced in CC from 5 hours onwards but this change did not correlate with GJ communication in the COC. The levels of Pfkp mRNA in CC were reduced after 5 hours, suggesting a significant reduction in glycolytic activity. Interestingly, mRNA levels of Cycs were also reduced in the CC although this did not occur until 25 hours. Levels of Gfpt 1 mRNA were upregulated in the CC at 13 hours, compared to 5 hours. Given the concurrent reduction in Cycs and Pfkp expression, these results suggest that glucose is directed to the hexosamine pathway at 13 hours, rather than the glycolytic pathway. The hexosamine biosynthetic pathway is involved in the production of substrates for $O$-linked glycosylation and in the synthesis of hyaluronic acid for CC expansion, while the glycolytic pathway is involved in energy production. Therefore, the results presented here suggest that CC energy production is reduced at 13 hours and that glucose is instead used for $O$-linked glycosylation and CC expansion, although CC expansion was not observed at any time point in this study (data not shown). Previously (Chapter 4), it was established that calcein-AM treatment prevented a time-dependent decrease in Cycs levels. It was also found that calcein-AM treatment resulted in increased Gfpt1 levels at 13 and 25 hours, and an increase in Atr mRNA at 13 hours. Therefore, the time dependent changes in Atr, Cycs, and Gfpt1 mRNA levels may be influenced by calcein-AM. However, the results presented here strongly support the notion that glucose metabolism is altered over time under in vitro conditions.

Western blotting was used to analyse changes in expression levels of CX43 and GDF9 in rat COC over time in culture. The Western blot protocol yielded variable results probably due to the limited number of replicate samples $(\mathrm{N}=2)$ able to be collected (i.e. each replicate sample consisted of $200 \mathrm{COC}$ ). Therefore, the results presented here are preliminary and further replicates need to be carried out in the future. Nevertheless, multiple bands were detected using the CX43-specific antibody, including a $43 \mathrm{kDa}$ band assumed to be CX43, and two unidentified bands around 63 and $22 \mathrm{kDa}$ in size. Interestingly, the $43 \mathrm{kDa}$ band and both of the unidentified bands were absent after the blot had been stripped and the CX43 
antibody added had been pre-absorbed with the control peptide. However, pre-absorption of the antibody with an antigen proves that the antibody binds to that antigen but does not prove the specificity of single bands (Swaab et al., 1977; Willingham, 1999). Thus, it is possible that the 63 and $22 \mathrm{kDa}$ bands were visualised as a result of nonspecific binding by the CX43 antibody. These bands may also represent differentially processed forms of CX43 protein however this has not been previously reported to the best of my knowledge. A 43 $\mathrm{kDa}$ band was notably absent in the rat aorta lysate positive control of the second replicate experiment. The phosphorylation pattern of CX43 is tissue specific (Kadle et al., 1991) thus CX43 from rat aorta may have a different migration pattern to that from the CC.

Multiple bands were also detected using the GDF9-specific antibody, including a $49 \mathrm{kDa}$ band assumed to be the promature protein, a $20 \mathrm{kDa}$ band assumed to be the mature protein (Lin et al., 2012), and unidentified bands of 90, 70, and $40 \mathrm{kDa}$ sizes. All of these bands were absent following stripping of the blot and re-probing with the GDF9-specific antibody that had been pre-absorbed with a recombinant ovine GDF9 protein. It is likely that some of these bands were differentially processed forms of GDF9. Indeed, a preproprotein also exists within the oocyte and is a non-secreted form. Furthermore, GDF9 contains five $\mathrm{N}$ linked glycosylation sites (Jaatinen et al., 1999) and is a target for phosphorylation (McMahon et al., 2008).

Analysis of the band densities was undertaken using the ImageJ software. The $43 \mathrm{kDa}$ band was analysed exclusively for the CX43 Western blots. For the GDF9 blots, the $49 \mathrm{kDa}$ and 20 kDa bands were analysed separately. The total protein content was measured in each sample using the BCA assay. The band densities for CX43 were corrected for total protein amount to reduce the variation caused by differences in CC number. The band densities for GDF9 were not corrected for total protein, to provide an indication of the total amount of protein produced by a known number of oocytes.

This study revealed that expression of CX43 protein in COC was increased at 13 hours in culture, compared to 1 and 5 hours, and the levels of CX43 at 25 hours were variable. As discussed previously, CX43 protein levels were not correlated to the pattern of GJ communication over time. This may be due to various GJ regulatory mechanisms, such as 
voltage gating, phosphorylation, and incorporation into GJ plaques, playing a major role in up- or down-regulating GJ communication.

Overall levels of promature GDF9 protein did not change over time. However, relatively large variability was observed in the levels of promature GDF9 measured in cultured COC at 5, 13, and 25 hours. The promature band was much denser than the mature band, indicating that the promature form is much more abundant in the cell than the mature form. A previous study found that the mature form of GDF9 was the predominant form detected in rat oocytes, and that promature protein was undetectable (Lin et al., 2012). However, there were significant experimental differences between the Lin et al. (2012) study and the experiments presented here. Most notably, the Lin et al. study measured GDF9 expression following 24 hours of culturing DO, as opposed to COC in this present study. Therefore, the presence of intact CC layers around the oocyte may alter the processing of uncleaved promature GDF9. The band representing the mature GDF9 protein was faint and highly variable in density between replicates. Although the mature form of the protein is the bioactive region, immunization against the proregion of GDF9 in mice resulted in ovaries with more corpora lutea and smaller litter sizes. This indicates that the GDF9 proregion may also play an important role in regulating fertility (Mclntosh et al., 2012). While this influence is likely to be through interactions between the cleaved proregion and the mature region (Mclntosh et al., 2008), this may also be as a result of undetermined biological activity of the uncleaved promature protein.

In summary, these results demonstrate that the communication pathways between the oocyte and CC remain active in rat COC throughout 49 hours of in vitro culture. In contrast to other species, levels of connexin mRNA (i.e. $C_{x} 43$ and $C_{x 3} 7$ ) or CX43 protein were not good indicators of functional GJ communication in rat COC. The levels of oocyte-derived mRNAs (with the exception of Atr) and GDF9 protein remained stable for up to 25 hours in culture. In the CC, expression levels of genes involved in the glycolytic pathway (i.e. Pfkp and Cycs) were reduced after 25 hours. However, Gfpt1 expression was similar at 25 hours compared to that at 1 hour. These results indicate that culturing rat $\mathrm{COC}$ in vitro may favour the hexosamine biosynthetic pathway, rather than the glycolytic pathway, for glucose utilisation. Moreover, these results validate the use of the GJ bioassay, qPCR and Western blotting 
methodologies to detect changes in key regulatory pathways that are involved in maintaining $\mathrm{COC}$ health. 


\section{EFFECTS OF IN VITRO EXPOSURE OF COC TO LIFESTYLE FACTORS ON CC-OOCYTE GJ COMMUNICATION}

\subsection{Introduction}

Maintenance of GJ communication within the COC is essential for oocyte development and maturation. The function of GJ are regulated at multiple levels including transcription, translation, post-translational modifications, incorporation into GJ plaques, voltage gating, and other slow gating processes (Bukauskas et al., 2000; Harris, 2002; Goodenough and Paul, 2009). Disruptions to any of these mechanisms have the potential to modify GJ communication. Moreover, suppression of GJ communication may result in the oocyte not receiving adequate nutrients for development and/or maturation (Kidder and Mhawi, 2002). In this context, blocking GJs between the CC and oocyte, using carbenoxolone, inhibited the transfer of a fluorescent glucose analogue (6-NBDG) and cAMP (Webb et al., 2002; Wang et al., 2012). Moreover, blocking GJs using octanol and heptanol resulted in a decreased proportion of bovine oocytes reaching the blastocyst stage of development (Atef et al., 2005). On the other hand, increasing GJ communication is likely to enhance the transfer of nutrients to the oocyte. This may be advantageous, due to more substrates entering the oocyte for energy production and maturation processes. Conversely, excessive GJ communication may also have a negative impact on oocyte quality by transferring more nutrients than necessary for oocyte growth, or may be indicative of a stressed oocyte requiring an increased need for energy-consuming RNA surveillance mechanisms (Baumann et al., 2007).

The closure of GJs between the oocyte and CC is necessary for normal nuclear maturation (Shimada et al., 2001; Norris et al., 2008). For example, suppression of inhibitory phosphatidylinositol 3 kinase mediated phosphorylation of CX43 in porcine COC resulted in a decreased proportion of MII oocytes following in vitro culture (Shimada and Terada, 2001). The timing of meiotic resumption is critical. Blastocyst formation was reduced in bovine oocytes pre-incubated with cyclohexamide (meiosis inhibitor) for 24 or 48 hours prior to IVM and fertilised under inhibitor-free conditions, compared to oocytes not exposed to meiotic inhibitors (Saeki et al., 1997). The effects of increased GJ communication on nutrient transfer to the oocyte have not been examined to the best of my knowledge. However, it is reasonable to assume that increased GJ communication results in more glucose and glucose 
metabolites being transferred to the oocyte, and indeed, this occurs under hyperglycaemic conditions (Wang et al., 2012). Hyperglycaemic conditions result in increased $O$-linked glycosylation (Brownlee, 2001; Schelbach et al., 2010), increased production of reactive oxygen species, and decreased glutathione concentrations in the oocyte (Hashimoto et al., 2000). Hyperglycaemic conditions also lead to an increased rate of oocyte meiotic maturation (Downs et al., 1998; Sutton-McDowall et al., 2005). Increased glucose concentrations are also correlated with increased FSH-induced CC expansion in bovine COC (Sutton-McDowall, 2004).

A number of environmental contaminants and lifestyle factors, including BPA, caffeine, and nicotine metabolites, have been found at measureable concentrations in follicular fluid as well as in serum of women (Zenzes et al., 1996; Ikezuki et al., 2002; Al-Saleh et al., 2010). Some of these compounds have also been reported to negatively affect female fertility (Emanuele et al., 2002; Klonoff-Cohen, 2005; Anderson, 2010). The environmental contaminant BPA and the lifestyle factors caffeine, ethanol and nicotine have all previously been reported to affect GJ communication in various tissue types (Flagg-Newton et al., 1981; Tsai et al., 2004; Mustonen et al., 2005; Lee and Rhee, 2007). However, the effects of physiological concentrations of these and other highly addictive and potentially detrimental compounds (e.g. MDMA and THC) on GJ communication within the COC have not been tested. Moreover, the large number of connexin isoforms, their combinations in differing tissue types and the unique regulatory properties of each isoform suggest that alterations in GJ communication in response to regulatory factors between tissue types will be unique.

The aims of this chapter were to determine the in vitro effects of high physiological concentrations of BPA, caffeine, ethanol, nicotine, MDMA, or THC with time on cultured COC in regards to (1) the degree of GJ communication between the oocyte and CC and (2) the timing of meiotic resumption of the oocyte.

\subsection{Methods}

\subsubsection{COC collection}

A full description of the $\mathrm{COC}$ collection procedure for the in vitro culture experiments is described in Section 2.1.2. In brief, ovaries were extracted, follicles were punctured with a 20 gauge needle, and morphologically healthy $\operatorname{COC}$ were transferred into 48 -well plates 
containing dissection media containing $50 \mu \mathrm{M}$ dipyridamole and $50 \mu \mathrm{M}$ rolipram. All COC were washed twice in wash media containing $50 \mu \mathrm{M}$ dipyridamole and $50 \mu \mathrm{M}$ rolipram.

\subsubsection{Exposure to lifestyle factors}

Stock solutions of each of the treatment reagents are listed in Table 6-1. The treatments were generally purchased from Sigma with the exception of BPA (donated by Phil Conveny; Nuplex Industries, NZ), MDMA (donated by Richard Moore; VUW), and THC (donated by Ryan Steel, VUW). The final concentrations chosen were based on levels that had been measured in biological fluids (see Table 1.1). Following the wash steps described above, COC were transferred into incubation media containing $50 \mu \mathrm{M}$ dipyridamole and $50 \mu \mathrm{M}$ rolipram without (control) or with (treated) the appropriate treatment. After 0, 4, 12, or 24 hours incubation at $37{ }^{\circ} \mathrm{C}$ in $5 \% \mathrm{CO}_{2}$, and $96 \%$ humidity, the $\mathrm{COC}$ were transferred into incubation media containing $2 \mu \mathrm{M}$ calcein- $\mathrm{AM}, 50 \mu \mathrm{M}$ dipyridamole and $50 \mu \mathrm{M}$ rolipram as well as the appropriate treatment for 10 minutes. The COC were then transferred into fresh incubation media (calcein-AM free) containing $50 \mu \mathrm{M}$ dipyridamole and $50 \mu \mathrm{M}$ rolipram and the appropriate treatment, and incubated for a further hour. The COC were washed twice in PBS and the oocyte was denuded of CC and measured for fluorescence as described previously. Thereafter, the oocyte was washed twice more in PBS. These individual DO and separated CC masses were suspended in $10 \mu \mathrm{L}$ PBS in the 96 -well plates and stored at $-80{ }^{\circ} \mathrm{C}$ until further analyses (see Chapter 2.1.3).

To reduce the number of samples, the experiments involving the treatments with caffeine and nicotine were combined, and the experiments involving the treatments with MDMA and THC were combined such that the treatments in each experiment were compared to one control group.

As described previously (Section 2.1.3.1), the raw fluorescence measurement for each individual oocyte was corrected for the geometric mean of the control group at 1 hour (i.e. those COC pre-incubated for 0 hours followed by a 1 hour post-calcein incubation). The data were tested for normality using the D'Agostino \& Pearson normality test and data that were not normally-distributed were transformed (square root or natural log transformations) and were assessed again for normality. The data were then analysed using a two-way ANOVA. 
Table 6-1: Stock and final concentrations of treatment reagents in media. With the exception of ethanol, which was stored in $\mathrm{dH}_{2} \mathrm{O}$, all other reagents were stored in DMSO.

\begin{tabular}{ccc}
\hline Treatment reagents & Stock concentration & Final Concentration \\
\hline BPA & $4 \mu \mathrm{g} / \mathrm{mL}$ & $20 \mathrm{ng} / \mathrm{mL}$ \\
Caffeine & $20 \mathrm{mg} / \mathrm{mL}$ & $100 \mu \mathrm{g} / \mathrm{mL}$ \\
Ethanol & $750 \mathrm{mg} / \mathrm{mL}$ & $7.5 \mathrm{mg} / \mathrm{mL}(0.95 \% \mathrm{w} / \mathrm{v})$ \\
Nicotine & $10 \mu \mathrm{g} / \mathrm{mL}$ & $500 \mathrm{ng} / \mathrm{mL}$ \\
MDMA & $400 \mu \mathrm{g} / \mathrm{mL}$ & $2 \mu \mathrm{g} / \mathrm{mL}$ \\
THC & $10 \mu \mathrm{g} / \mathrm{mL}$ & $500 \mathrm{ng} / \mathrm{mL}$ \\
\hline
\end{tabular}

\subsection{Results}

\subsubsection{Effect of BPA on GJ communication in rat COC}

The effect of exposure of rat COC to $20 \mathrm{ng} / \mathrm{mL}$ BPA in vitro on GJ communication over time is depicted in Figure 6-1. A significant effect of BPA treatment on oocyte fluorescence was noted, but no effects of time or treatment $x$ time interaction were observed. Although no significant effect of time was measured, oocyte fluorescence appeared marginally increased in the control COC at 13 hours, compared to 1 hour, but was decreased at 25 hours. This reduction in oocyte fluorescence in the control $\mathrm{COC}$ at 25 hours was not mirrored in the BPA-treated COC. Instead, mean oocyte fluorescence in the BPA-treated COC increased progressively at each consecutive time point, although no significant effect of time was demonstrated. The proportion of oocytes with a visible GV declined over time in both the control and BPA-treated groups (Table 6-2). There was no difference in the proportion of oocytes with a visible GV between the control and BPA-treated groups. 


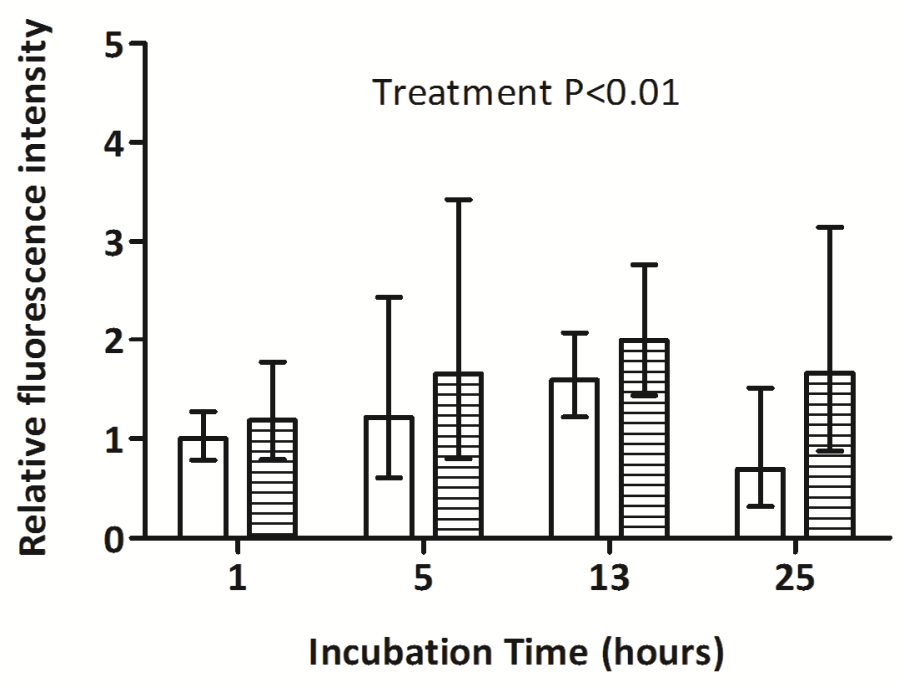

Figure 6-1: Effect of BPA on fluorescent dye transfer from adjacent CC to oocytes over time in vitro. The incubation time indicates the pre-calcein incubation + the 1 hour post-calcein incubation. The control (i.e. untreated) group is represented by the unfilled bars ( $\square$ ), while the BPA-treated group is represented by the hatched bars ( Values are shown as geometric means $\pm 95 \%$ confidence intervals of fluorescent ratios normalised against the geometric mean of the control group at 1 hour. The data represent 11-12 readings from individual oocytes in each group generated from 3 replicate experiments. The data were transformed (square root) prior to being analysed by a two-way ANOVA. The mean \pm SEM fluorescent reading in the control group at 1 hour was $167 \pm 39$.

Table 6-2: Effect of BPA on the proportion of oocytes with a visible GV. The table shows the proportion of oocytes with a visible GV (\%), with the actual number of oocytes with a GV in brackets. The data from 3 replicate experiments ( $\mathrm{N}=11-12 /$ group) were analysed by Fisher's exact test. No effect of BPA on the presence of a GV was noted.

\begin{tabular}{ccc}
\hline Time & Control & BPA \\
\hline $\mathbf{1}$ & $100(12)$ & $75(9)$ \\
$\mathbf{5}$ & $42(5)$ & $75(9)$ \\
13 & $67(8)$ & $58(7)$ \\
25 & $42(5)$ & $50(6)$ \\
\hline
\end{tabular}

\subsubsection{Effect of caffeine on GJ communication in rat COC}

The effect of exposure of rat $\mathrm{COC}$ to $100 \mu \mathrm{g} / \mathrm{mL}$ caffeine in vitro on GJ communication over time is depicted in Figure 6-2. Overall, there were no significant effects of treatment, time or treatment $x$ time interaction. It is worth noting that the treatment effect almost reached 
significance $(P=0.052)$. Mean oocyte fluorescence in the control COC remained essentially unchanged for the first 13 hours but by 25 hours, a lower (albeit not significant) mean level of fluorescence was evident. This reduction in oocyte fluorescence at 25 hours was not observed in the caffeine-treated COC. Instead, the mean oocyte fluorescence levels in the caffeine-treated COC were essentially unchanged over 25 hours. The proportion of oocytes with a visible GV declined over time in both the control and caffeine-treated groups (Table 6-3). There was no difference in the proportion of oocytes with a visible GV between the control and caffeine-treated groups.

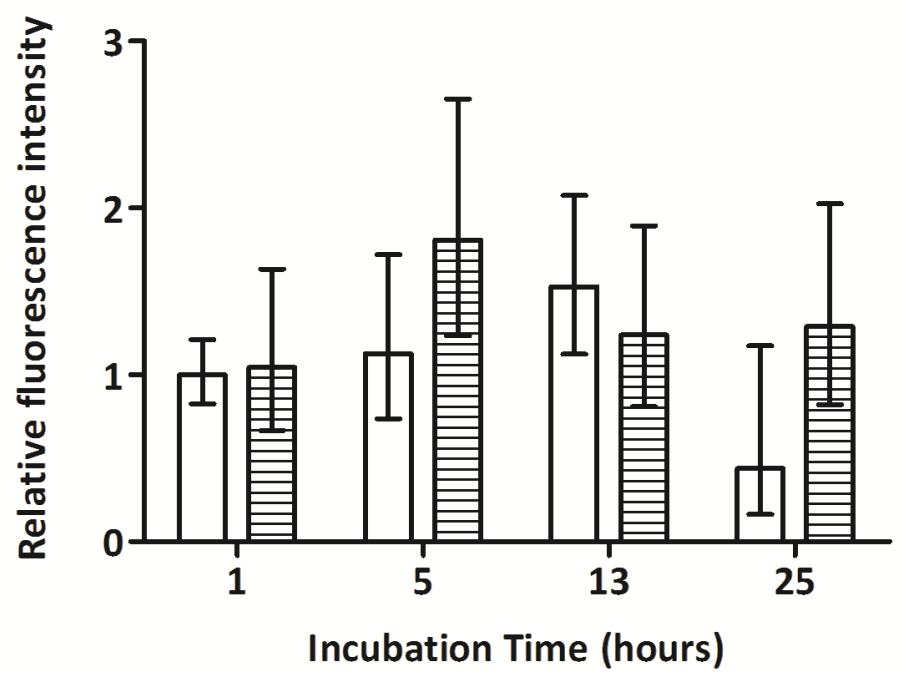

Figure 6-2: Effect of caffeine on fluorescent dye transfer from adjacent CC to oocytes over time. The incubation time indicates the pre-calcein incubation + the 1 hour post-calcein incubation. The control (i.e. untreated) group is represented by the unfilled bars ( $\square$ ), while the caffeine-treated group is represented by the hatched bars ( normalised against the geometric mean of the control group at 1 hour. The data represent 10-12 readings from individual oocytes in each group generated from 3 replicate experiments. The data were transformed (square root) and then analysed by a two-way ANOVA. The mean \pm SEM fluorescent reading in the control group at 1 hour was $224 \pm 24$. 
Table 6-3: Effect of caffeine on the proportion of oocytes with a visible GV. The table shows the proportion of oocytes with a visible GV (\%), with the actual number of oocytes with a GV in brackets. The data from 3 replicate experiments ( $\mathrm{N}=10-12 /$ group) were analysed by a Fisher's exact test. No effect of caffeine on the presence of a GV was noted.

\begin{tabular}{ccc}
\hline Time & Control & Caffeine \\
\hline 1 & $83(10)$ & $83(10)$ \\
5 & $75(9)$ & $75(9)$ \\
13 & $42(5)$ & $67(8)$ \\
25 & $42(5)$ & $58(7)$ \\
\hline
\end{tabular}

\subsubsection{Effect of ethanol on GJ communication in rat COC}

The effect of exposure of rat $\mathrm{COC}$ to $7.5 \mathrm{mg} / \mathrm{mL}$ ethanol in vitro on GJ communication over time is depicted in Figure 6-3. No effects due to treatment or time, or treatment $x$ time interaction were observed. The proportion of oocytes with a visible GV decreased over time in both the control and ethanol-treated groups (Table 6-4). When the data from all time points were pooled, there was a greater proportion of oocytes with a visible GV in the ethanol-treated group compared to the control group $(P<0.05)$. 


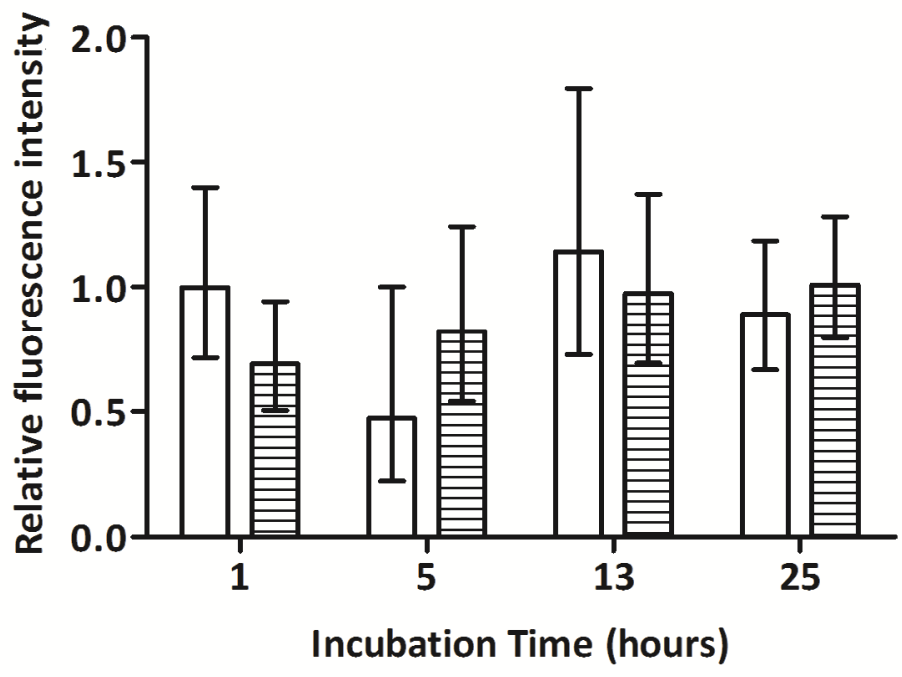

Figure 6-3: Effect of ethanol on fluorescent dye transfer from adjacent CC to oocytes over time in vitro. The incubation time indicates the pre-calcein incubation + the 1 hour post-calcein incubation. The control (i.e. untreated) group is represented by the unfilled bars ( $\square$ ), while the ethanol-treated group is represented by the hatched bars ( $)$ ). Values are shown as geometric means $\pm 95 \%$ confidence intervals of fluorescent ratios normalised against the geometric mean of the control group at 1 hour. The data represent 9-12 readings from individual oocytes in each group generated from 3 replicate experiments. The data were transformed (square root) and analysed by a two-way ANOVA. The mean \pm SEM fluorescent reading in the control group at 1 hour was $313 \pm 71$.

Table 6-4: Effect of ethanol on the proportion of oocytes with a visible GV. The table shows the proportion of oocytes with a visible GV, with the actual number of oocytes with a GV in brackets. The data from 3 replicate experiments ( $\mathrm{N}=9-12 /$ group) were analysed by a Fisher's exact test.

\begin{tabular}{ccc}
\hline Time & Control & Ethanol \\
\hline $\mathbf{1}$ & $62(7)$ & $83(10)$ \\
$\mathbf{5}$ & $50(6)$ & $75(9)$ \\
13 & $29(4)$ & $75(9)$ \\
25 & $33(4)$ & $42(5)$
\end{tabular}

\subsubsection{Effect of nicotine on GJ communication in rat COC}

The effect of exposure of rat $\mathrm{COC}$ to $500 \mathrm{ng} / \mathrm{mL}$ nicotine in vitro on GJ communication over time is depicted in Figure 6-4. No significant effects of treatment or time were detected but a significant treatment $x$ time interaction was noted. The mean oocyte fluorescence in the 
control COC remained essentially unchanged over the first 13 hours but appeared to be lower at 25 hours. This lower (albeit not significant) level at 25 hours in the control group was not evident in the nicotine-treated group where the mean fluorescence levels remained essentially unchanged over the 25 hour period. There was no difference in the proportion of oocytes with a visible GV between the control and nicotine-treated groups (Table 6-5).

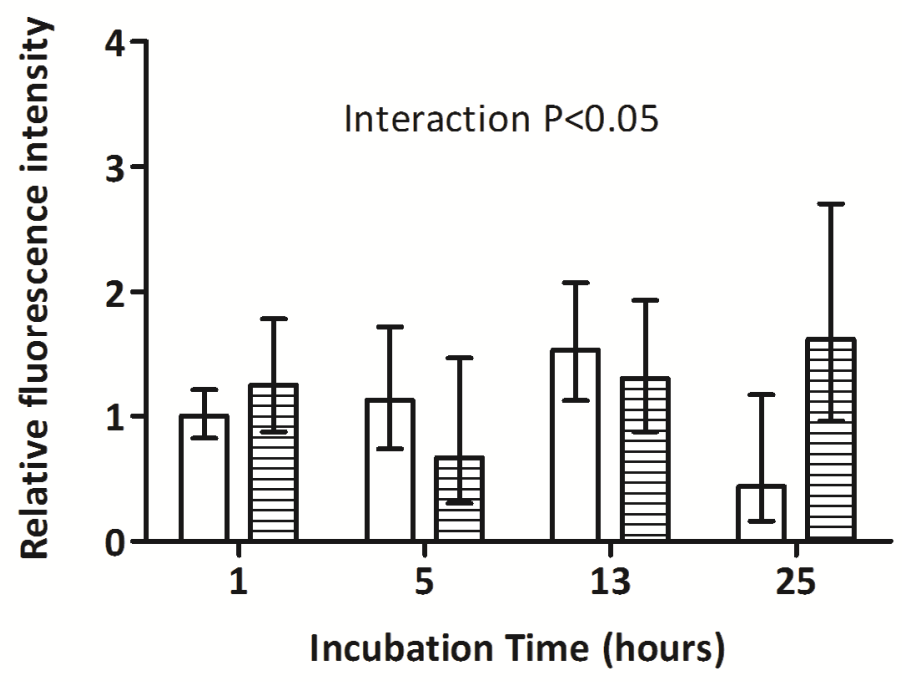

Figure 6-4: Effect of nicotine on fluorescent dye transfer from adjacent CC to oocytes over time in vitro. The incubation time indicates the pre-calcein incubation + the 1 hour post-calcein incubation. The control (i.e. untreated) group is represented by the unfilled bars ( $\square$ ), while the nicotine-treated group is represented by the hatched bars (局). Values are shown as geometric means $\pm 95 \%$ confidence intervals of fluorescent ratios normalised against the geometric mean of the control group at 1 hour. The data represent 12 readings from individual oocytes in each group generated from 3 replicate experiments. The data were transformed (square root) and analysed by a two-way ANOVA. The mean \pm SEM fluorescence reading in the control group at 1 hour was $224 \pm 24$.

Table 6-5: Effect of nicotine on the proportion of oocytes with a visible GV. The table shows the proportion of oocytes with a visible GV (\%), with the actual number of oocytes with a GV in brackets. The data from 3 replicate experiments ( $N=12 /$ group) were analysed by a Fisher's exact test. No effect of nicotine on the presence of a GV was noted.

\begin{tabular}{ccc}
\hline Time & Control & Nicotine \\
\hline $\mathbf{1}$ & $83(10)$ & $67(8)$ \\
13 & $75(9)$ & $67(8)$ \\
25 & $42(5)$ & $42(5)$ \\
& $42(5)$ & $67(8)$ \\
\hline
\end{tabular}




\subsubsection{Effect of MDMA on GJ communication in rat COC}

The effect of exposure of rat $\mathrm{COC}$ to $2 \mu \mathrm{g} / \mathrm{mL}$ MDMA in vitro on $\mathrm{GJ}$ communication over time is shown in Figure 6-5. A significant effect of time but not treatment was noted. Moreover, there was no significant treatment $\mathrm{x}$ time interaction. Overall, mean oocyte fluorescence was lower at 13 and 25 but not 5 hours in both the control and MDMA-treated groups. The proportion of oocytes with a visible GV decreased over time in both the control and MDMAtreated groups (Table 6-6). When observations from all time points were pooled, there was a lower proportion of oocytes with a visible GV in the MDMA-treated group compared to the control group $(\mathrm{P}<0.001)$.

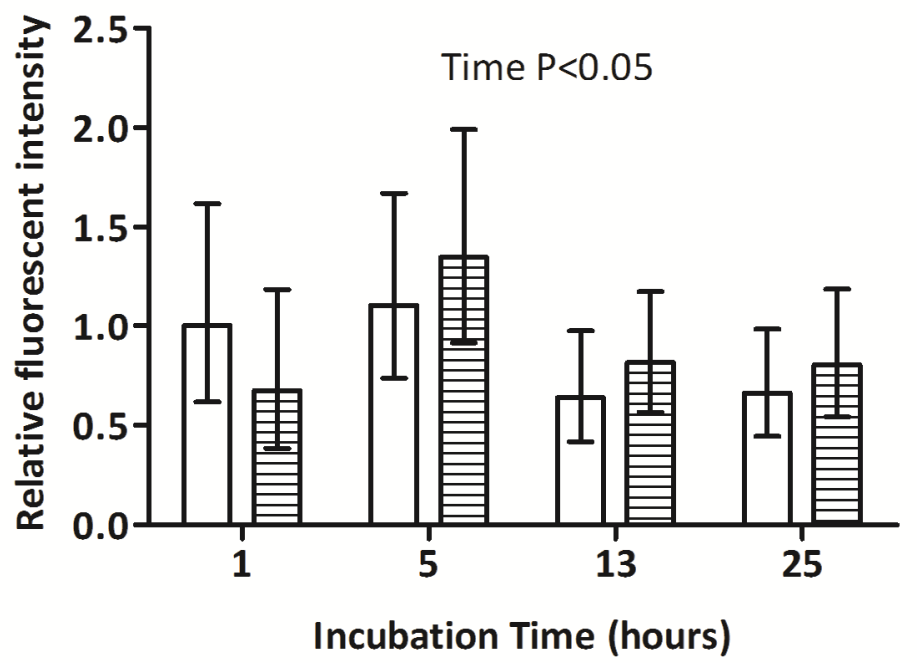

Figure 6-5: Effect of MDMA on fluorescent dye transfer from adjacent CC to oocytes over time. The incubation time indicates the pre-calcein incubation +1 hour the post-calcein incubation. The control (i.e. untreated) group is represented by the unfilled bars ( $\square$ ), while the MDMA-treated group is represented by the hatched bars (局). Values are shown as geometric means $\pm 95 \%$ confidence intervals of fluorescent ratios normalised against the geometric mean of the control group at 1 hour. The data represent 11-12 readings from individual oocytes in each group generated from 3 replicate experiments. The data were transformed (square root) and analysed by a two-way ANOVA. The mean \pm SEM fluorescent reading in the control group at 1 hour was $139 \pm 31$. 
Table 6-6: Effect of MDMA on the proportion of oocytes with a visible GV. The table shows the proportion of oocytes with a visible GV (\%), with the actual number of oocytes with a GV in brackets. The data from 3 replicate experiments ( $\mathrm{N}=11-12 /$ group) were analysed by a Fisher's exact test.

\begin{tabular}{ccc}
\hline Time & Control & MDMA \\
\hline $\mathbf{1}$ & $92(11)$ & $92(11)$ \\
$\mathbf{5}$ & $83(10)$ & $25(3)$ \\
$\mathbf{1 3}$ & $67(8)$ & $25(3)$ \\
$\mathbf{2 5}$ & $58(7)$ & $17(2)$ \\
\hline
\end{tabular}

\subsubsection{Effect of THC on GJ communication in rat COC}

The effect of exposure of rat $\mathrm{COC}$ to $500 \mathrm{ng} / \mathrm{mL}$ THC in vitro on $\mathrm{GJ}$ communication over time is depicted in Figure 6-6. Overall, there was a significant effect of incubation time but no effect of treatment and no treatment $x$ time interaction. Mean oocyte fluorescence was lower at 13 and 25 hours in both the control and THC-treated groups. The proportion of oocytes with a visible GV declined over time in both the control and THC-treated groups (Table 6-7). When observations from all time points were pooled, the difference in the proportion of oocytes with a visible GV between the control and THC-treated groups almost reached significance $(P=0.054)$. When observations from the 5,13 , and 25 hour time points were pooled, the proportion of oocytes with a visible GV was significantly reduced in the THC-treated group compared to the control $(P<0.05)$. 


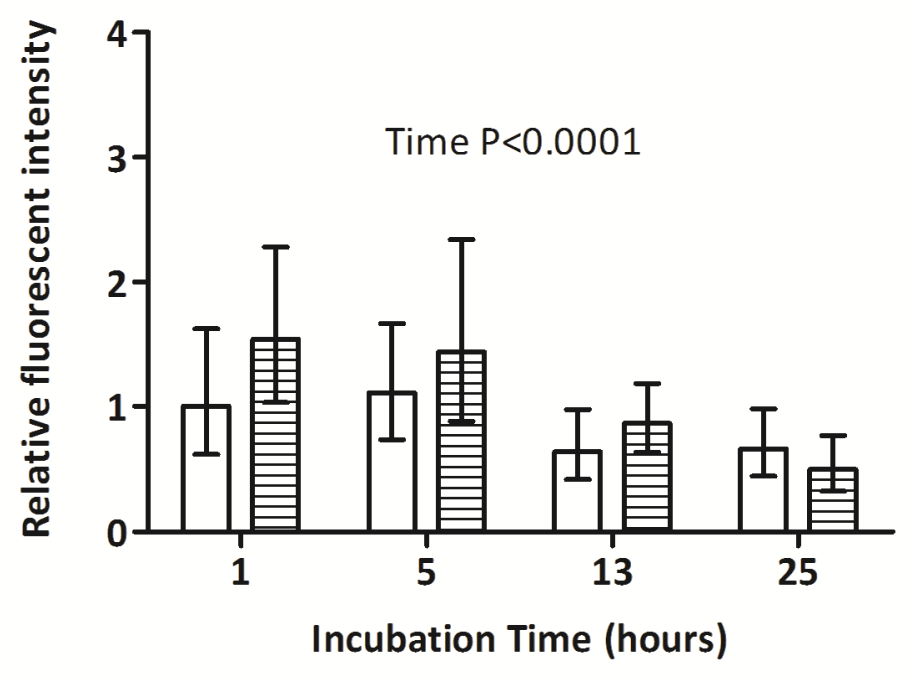

Figure 6-6: Effect of THC on fluorescent dye transfer from adjacent CC to oocytes over time in vitro. The incubation time indicates the pre-calcein incubation + the 1 hour post-calcein incubation. The control (i.e. untreated) group is represented by the unfilled bars ( $\square$ ), while the THC-treated group is represented by the hatched bars ( normalised against the geometric mean of the control group at 1 hour. The data represent 12 readings from individual oocytes in each group generated from 3 replicate experiments. The data were transformed (natural $\log$ ) and analysed by a two-way ANOVA. The mean \pm SEM fluorescent reading in the control group at 1 hour was $139 \pm 31$.

Table 6-7: Effect of THC on proportion of oocytes with a visible GV. The table shows the proportion (\%) of oocytes with a visible GV, with the actual number of oocytes with a GV in brackets. The data from 3 replicate experiments ( $N=12 /$ group) were analysed by a Fisher's exact test.

\begin{tabular}{ccc}
\hline Time & Control & THC \\
\hline 1 & $92(11)$ & $92(11)$ \\
5 & $83(10)$ & $42(5)$ \\
13 & $67(8)$ & $50(6)$ \\
25 & $58(7)$ & $33(4)$
\end{tabular}

\subsection{Discussion}

The focus of this study was to investigate the effects of several lifestyle factors and environmental contaminants on the $\mathrm{COC}$ communication system and hence their influence oocyte health. The results reveal that there was no treatment effect noted after in vitro 
exposure of rat $\mathrm{COC}$ to high physiological concentrations of caffeine, ethanol, MDMA or THC on fluorescent dye transfer over 25 hours relative to the controls. It is possible that there is an effect of caffeine as this treatment approached significance. However, further studies are needed to confirm whether this is of biological significance. In contrast, exposure to nicotine resulted in a treatment $x$ time interaction, as a result of a dramatic decline in dye transfer that was noted in the control group after 13 hours whereas no change in dye transfer over 25 hours was measured in the nicotine-treated group. The only treatment effect independent of time in vitro was noted for BPA where the pattern of fluorescent dye transfer was similar to that for nicotine: again no change was noted for the treated group over 25 hours whereas a dramatic decline after 13 hours was noted for the control. Thus, exposure of COC to BPA and nicotine may require oocytes to demand a higher level of CCderived factors to mitigate the possible negative effects of these reagents. With respect to measures of oocyte maturation status (i.e. the presence of a GV) after exposure to lifestyle factors, it was noted that in vitro exposure to ethanol suppressed oocyte meiotic maturation, whereas in vitro exposure to MDMA or THC enhanced the proportion of oocytes undergoing GVBD.

In general, the compounds tested in the present study were at concentrations 10-fold higher than that measured previously in biological fluids. These concentrations were chosen as they were above the normal physiological range, but were not so high to induce a false positive result. The concentrations of those compounds that had not been measured previously in follicular fluid were determined based on the concentration in blood. In general, the concentrations of small molecules are comparable between plasma and follicular fluid (Edwards, 1974).

The exposure of rat COC to BPA caused an overall increase in GJ communication. The largest difference in oocyte fluorescence between the treated and control COC occurred after 25 hours in culture. The reduction in oocyte fluorescence that occurred in control COC after a 25 hour culture period was not observed in those COC exposed to BPA. This observation suggests that BPA prevents closure of the GJs. Interestingly, previous in vitro studies using a higher dose of BPA found that BPA suppressed GJ communication in an epithelium-derived rat breast cancer cell line in vitro (Lee and Rhee, 2007). Similarly, BPA has also been reported to suppress spontaneous GJ-dependent $\mathrm{Ca}^{2+}$ oscillations in mouse oocytes (Mohri and 
Yoshida, 2005). However, it is important to note that the concentrations of BPA used in these studies were between 4- and 1000-fold higher than that used in the present study. Whilst the concentration used in this study was lower, it was nevertheless above the normal physiological range. In future studies it would be of interest to test BPA concentrations within the expected physiological range. As discussed previously, the consequence of increased GJ communication may include increased transfer of cAMP, glucose metabolites, and other nutrients to the oocyte. BPA has previously been reported to reduce oocyte quality in both mice and humans (Eichenlaub-Ritter et al., 2008; Fujimoto et al., 2010). Furthermore, in vitro exposure to BPA did not affect oocyte nuclear maturation, as the proportion of oocytes undergoing GVBD was unchanged following exposure to BPA for up to 25 hours. It should be noted here that the number of samples used in this study was small resulting in the inability to detect small differences between groups. While the Fisher's exact test is more sensitive than the traditional chi-square test, it still lacks power with small sample sizes (Upton, 1992). Previous reports indicate that BPA does promote meiotic arrest in oocytes. For example, a greater proportion of mouse oocytes underwent GVBD following exposure to $10 \mu \mathrm{g} / \mathrm{mL}$, but not lower concentrations, of BPA (Eichenlaub-Ritter et al., 2008). Additionally another study revealed that exposure of mouse oocytes to BPA was associated with a decreased ability of oocytes to resume meiosis, and in those that do, an increased proportion arrest after GVBD and fail to extrude a first polar body (Lenie et al., 2008). Again, both of these studies used much higher concentrations of BPA than that used in this study (340-fold and 500-fold higher respectively). Interestingly, the former study found that lower concentrations of BPA did not affect meiotic maturation of the oocyte even after 16 hours of exposure to BPA. Therefore the results from the present study are in agreement with previous publications that determined that high physiological concentrations of BPA do not affect oocyte meiotic maturation.

Overall, caffeine appeared to promote GJ communication in rat $\mathrm{COC}$ at some time points although this did not quite reach significance. Similar to BPA-treated oocytes, the largest difference in oocyte fluorescence between the caffeine-treated and control $\mathrm{COC}$ occurred after 25 hours in culture. Additionally oocyte fluorescence was highest in the caffeinetreated $\mathrm{COC}$ at 5 hours. Again, the reduction in oocyte fluorescence that occurred in control $\mathrm{COC}$ after a 25 hour culture period was not observed in those $\mathrm{COC}$ exposed to caffeine. This 
is consistent with previous reports that caffeine increased GJ communication in rat fibroblasts and epithelial cells in vitro (Flagg-Newton et al., 1981). These results suggest that caffeine may affect oocyte quality by preventing closure of the GJs. In vitro exposure to caffeine has also been reported to increase the glutathione content of ovine oocytes, and to improve the embryo cleavage rate (Ye et al., 2010). However, evidence regarding the effect of caffeine consumption on female fertility and oocyte quality in humans is inadequate and controversial (Anderson, 2010). Interestingly in the current study, caffeine did not have a significant effect on the proportion of oocytes that underwent GVBD over time. Caffeine is a broad-range PDE inhibitor (Rahimi et al., 2010), that has been shown to maintain meiotic arrest in mouse and porcine oocytes (Jagiello et al., 1972; Kren et al., 2004). However in contrast to the current study, neither of these studies added other specific PDE inhibitors into the culture medium. The use of rolipram and dipyridamole in this study may have inhibited the relevant PDEs to a maximal level, thus potentially masking the PDE inhibitory effects of caffeine.

The exposure of rat $\mathrm{COC}$ to ethanol had no overall effects on GJ communication. A study investigating rat gastric mucosal cells showed that ethanol reduced GJ communication, although a higher dose ( 8 fold) was used than that in the present study (Mustonen et al., 2005). It is entirely plausible that the incubation conditions may cause ethanol to evaporate out of the media, causing variable or false negative results. However, this has been tested in other studies and $96 \pm 2 \%$ of the added ethanol remained in the media following a 25 hour incubation at $37^{\circ} \mathrm{C}$ (Messing et al., 1986). Interestingly, ethanol appeared to inhibit oocyte meiotic maturation, as the proportion of oocytes that underwent GVBD was significantly lower over time in the ethanol-treated group compared to the control. A previous study involving the exposure of ovulated mouse oocytes to $7 \%$ ethanol reported a stimulation of parthenogenetic activation (i.e. progression of meiosis from metaphase II to completion), as measured by the number of unfertilized oocytes that had extruded a second polar body (O'Neill et al., 1989). This suggests that ethanol modulates progression of meiosis II in oocytes. However to the best of my knowledge the effect of ethanol on meiosis I (i.e. prior to ovulation) has not been investigated.

Nicotine had no overall effect on GJ communication in rat $\mathrm{COC}$ however there was a significant treatment $x$ time interaction. The reduction in oocyte fluorescence that occurred 
in control COC after a 25 hour culture period was not observed in those COC exposed to nicotine, as was also the case for BPA and caffeine. These results are in contrast to previous studies that found that in vitro exposure to nicotine resulted in decreased GJ communication in human umbilical vein endothelial cells (Tsai et al., 2004). However, the concentration of nicotine used in the study was 195 -fold greater than that used in the current study. It is important to note that nicotine is rapidly metabolised into the two major metabolites, cotinine and nicotine- $N$-oxide by the enzymes cytochrome p450 2A6 (CYP2A6) and flavincontaining monooxygenase 3 (FMO3), respectively (Benowitz, 1986; Hukkanen et al., 2005). The enzyme CYP2A6 has been detected in mouse ovaries (Cannady et al., 2003) and cotinine has been measured at concentrations of $\sim 700 \mathrm{ng} / \mathrm{mL}$ in follicular fluid and $50-200 \mathrm{ng} / \mathrm{mL}$ in plasma of active smokers (Zenzes et al., 1996; Shoaib and Stolerman, 1999). To my knowledge, there is no available data on the activity of FMO3 or the levels of nicotine- $\mathrm{N}$ oxide in the ovary. Nicotine did not have a significant effect on the proportion of oocytes that underwent GVBD over time. In contrast to this study, nicotine has previously been reported to promote oocyte nuclear maturation in bovine oocytes (Liu et al., 2008). However, the Liu study used a concentration of nicotine more than 1200 -fold greater than was used in the current study.

The effects of the active ingredients in two illicit drugs (i.e. MDMA and THC) on GJ communication in $\mathrm{COC}$ was also tested for the first time in this study. Exposure to MDMA did not affect the ability of the oocyte to import dye from the CC. Interestingly though, meiotic maturation of the oocyte was affected by MDMA. Significantly, fewer oocytes with a visible GV were present in the MDMA-treated group compared to the controls. These results suggest that MDMA prematurely advances meiotic progression in rat COC. In the present study, exposure of $\mathrm{COC}$ to THC also had no effect on transfer of calcein from the CC to the oocyte compared to the controls. A previous study found that in vitro exposure to THC inhibited GJ communication in a rat liver epithelial cell line (Upham et al., 2003). However, the concentrations used were $\sim 8$-fold greater than in the present study. The results in this study indicate that exposure to THC may prematurely advance oocyte meiotic maturation, as there were fewer oocytes remaining with a visible GV after exposure to THC compared to the controls. This in agreement with a previous study that found that exposure of rat follicles to THC in vitro induced resumption of meiosis (Reich et al., 1982). 
The study design for the GJ bioassay meant that COC were incubated for $0,4,12$, or 24 hours in the presence or absence of the appropriate treatment followed by incubation with calcein-AM and a subsequent 1 hour post calcein-AM incubation with or without treatment. Incubating COC with the lifestyle factors for up to 24 hours prior to calcein-AM exposure allowed for identification of factors that slowly regulated GJ communication. The 1 hour post calcein-AM incubation was carried out to allow for transfer of the fluorescent dye. Performing the post calcein-AM incubation in the absence of treatment may have released the regulatory action of that treatment, providing a false negative result. Thus, the post calcein-AM incubation was carried out in the presence or absence of treatment, as appropriate.

In summary, this in vitro study found that exposure to $20 \mathrm{ng} / \mathrm{mL}$ BPA and $500 \mathrm{ng} / \mathrm{mL}$ nicotine prevented a time-dependent decrease of GJ communication in rat COC. A reduction in oocyte fluorescence was measured in the control group after 25 hours but not in the BPA or nicotine-treated COC. Moreover, exposure of $\operatorname{COC}$ to $100 \mu \mathrm{g} / \mathrm{mL}$ caffeine in vitro may promote GJ communication, however this effect was negligible. In contrast, exposure to 7.5 $\mathrm{mg} / \mathrm{mL}$ ethanol, $2 \mu \mathrm{g} / \mathrm{mL}$ MDMA or $500 \mathrm{ng} / \mathrm{mL}$ THC did not affect GJ communication in rat COC. In vitro exposure to ethanol inhibited oocyte meiotic maturation, as measured by the proportion of oocytes with a visible GV, while exposure to MDMA or THC promoted oocyte meiotic maturation. None of the other treatments affected the proportion of oocytes with a visible GV. 


\section{EFFECTS OF IN VITRO EXPOSURE OF COC TO LIFESTYLE FACTORS ON GENE EXPRESSION IN OOCYTES}

\subsection{Introduction}

Oocyte competency, defined as the ability of an oocyte to develop into a viable embryo, is dependent upon a two-way communication system between the oocyte and $\mathrm{CC}$ that facilitates a co-ordinated maturation process of all follicular constituents (Gilchrist et al., 2008). The oocyte indirectly regulates its own growth and maturation through secretion of oocyte growth factors that act on the CC. The key oocyte growth factors GDF9 and BMP15 regulate important developmental events in GC (e.g. proliferation, steroidogenesis and differentiation, prevention of premature luteinisation and apoptosis, and CC mucification) as well as the synthesis and delivery of essential reagents and substrates from the $G C$ to the oocyte (Gilchrist et al., 2008; Su et al., 2009). Levels of mature GDF9 in follicular fluid have been reported to be positively associated with high quality embryos during IVF (Gode et al., 2011). A number of other processes are also indicative of oocyte competence including glucose uptake and metabolism (Krisher and Bavister, 1999), DNA damage repair (Marangos and Carroll, 2012), and mitochondrial function (Stojkovic et al., 2001).

Within an antral follicle, the $\mathrm{CC}$ that surround the oocyte are in direct contact with follicular fluid. In humans, numerous studies have shown that after exposure to certain chemical contaminants such as BPA, caffeine or cotinine, these reagents are able to cross from the blood stream into follicular fluid. In this context, these contaminants have the potential to influence oocyte functions (Zenzes et al., 1996; Ikezuki et al., 2002; Al-Saleh et al., 2010). Moreover, results from Chapter 6 established that in vitro exposure of rat COC to BPA or nicotine resulted in increased trafficking from CC to the oocyte via GJ communication. Conversely, these results also showed that in vitro exposure to caffeine, ethanol, MDMA, or THC did not affect GJ communication in rat COC. While transfer of nutrients to the oocyte is important for oocyte maturation, this does not provide information about the health of the oocyte itself. Oocyte health may be indirectly measured by quantifying the expression levels of key genes involved in critical pathways that regulate normal oocyte function. 
The aim of this chapter was to determine the effects of in vitro exposure of rat COC to BPA, caffeine, ethanol, nicotine, MDMA, or THC on Atr, Bmp15, Cx37, and Gdf9 mRNA expression levels within the oocyte.

\subsection{Methods}

DO were collected at completion of a GJ bioassay (those samples collected for Chapter 6). The samples were stored at $-80^{\circ} \mathrm{C}$ prior to extraction of total RNA as described in Section 2.3.1. The mRNA levels of Atr, Cycs, Glut1; and Gdf9, Bmp15 and Cx37 were measured using multiplex TaqMan qPCR as previously described (Section 2.3.2). Levels of Cycs and Glut1 were below the detection limit, and were not included in the analyses. Expression levels of the reference gene Rp/19 were also measured in each of these multiplex reactions. Each reaction run included a calibrator sample which contained cDNA from a pool of rat COC and this allowed comparison between reaction runs. The mRNA levels for each candidate gene were corrected for the mRNA levels of the same gene in the calibrator sample. Subsequently, the data were analysed using the $2^{\left(-\Delta \Delta C_{T}\right)}$ method (Livak and Schmittgen, 2001). The relative linear mRNA levels were analysed for normality using the D'Agostino \& Pearson normality test. Data that were not normally-distributed were transformed (using natural logarithms, squared, or square root transformations) and reanalysed by the D'Agostino \& Pearson normality test to confirm a normal distribution. Normally-distributed data were analysed by a two-way ANOVA.

\subsection{Results}

\subsubsection{Effect of BPA on gene expression in rat oocytes}

The effect of in vitro exposure of rat COC to $20 \mathrm{ng} / \mathrm{mL}$ BPA on mRNA levels of oocytes is depicted in Figure 7-1. For all candidate genes (i.e. Atr, Bmp15, Cx37 and Gdf9), there were no significant effects of time or treatment although a treatment $x$ time interaction was close to being significant for $C \times 37(P=0.058)$. This appeared to be due to the mean $C \times 37$ levels decreasing over time in the BPA-treated oocytes, whilst that in the control oocytes appeared to increase. Very similar patterns of gene expression were observed for Atr, Bmp15, and Gdf9. There was a significant effect of time but not treatment on Rp/19 mRNA levels and no treatment $x$ time interaction was observed. Overall, the levels of Rp/19 mRNA were lower at 25 hours compared to 1 hour. 
A

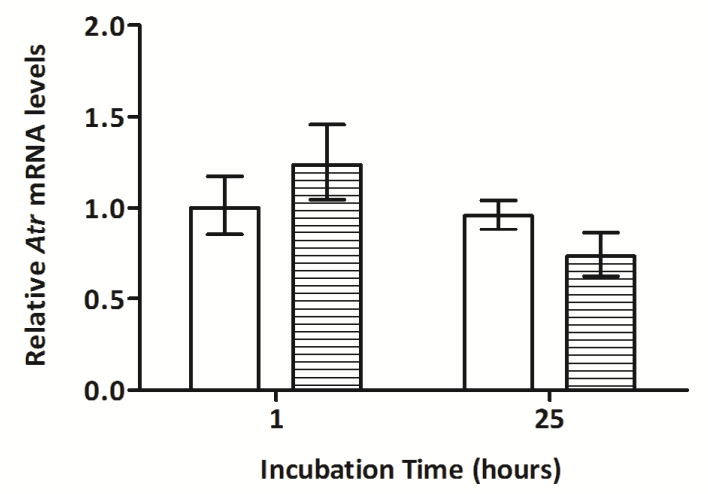

C

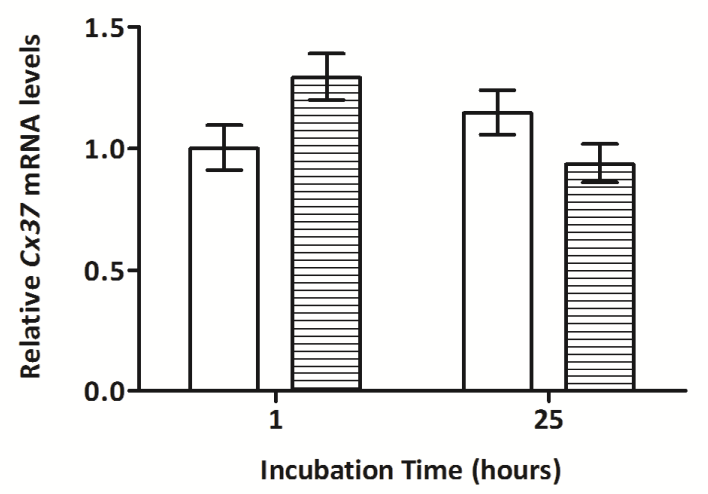

$\mathrm{E}$

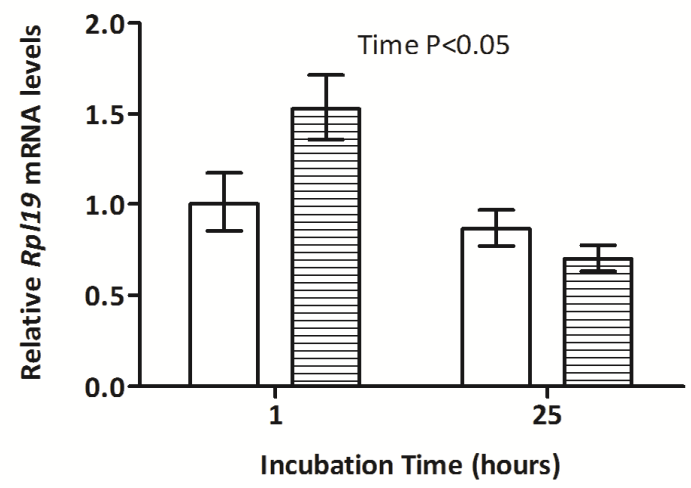

B

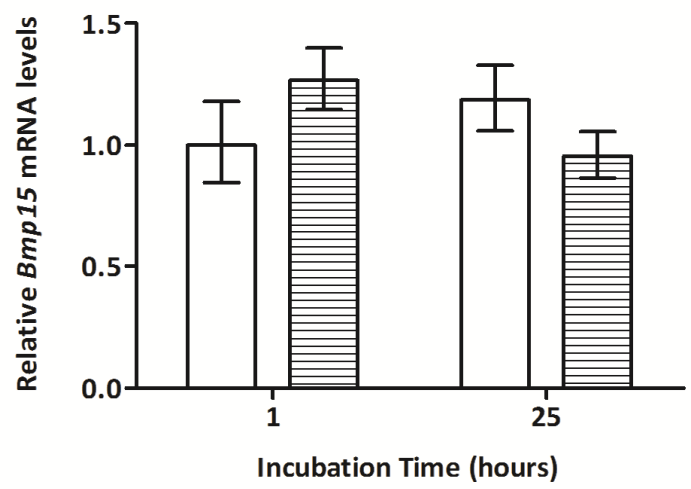

D

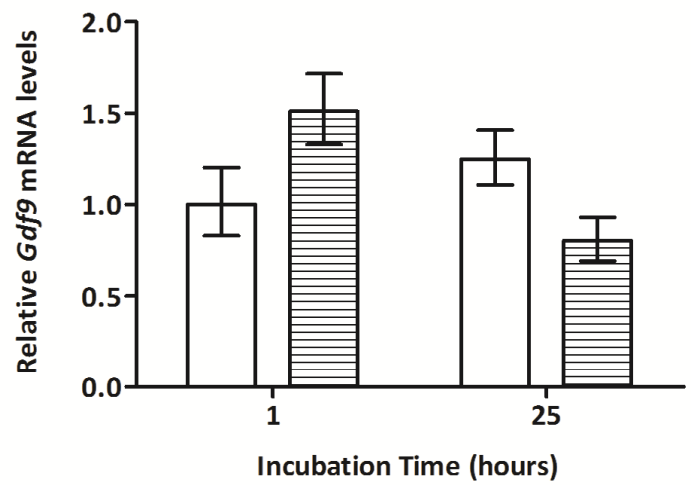

Figure 7-1: Effect of BPA on mRNA levels of Atr (A), Bmp15 (B), Cx37 (C), Gdf9 (D), and Rpl19 (E) in rat oocytes. The control group is represented by the unfilled bars $(\square)$, while the BPA-treated group is represented by the hatched bars ( normalised against the mean of the control group at 1 hour. The data were generated from a total of 10-12 readings from individual oocytes in each group. A two-way ANOVA was performed on untransformed data ( $B$, C), natural log transformed data (A, E) or squared data (D).

\subsubsection{Effect of caffeine on gene expression in rat oocytes}

The effect of in vitro exposure of rat COC to $100 \mu \mathrm{g} / \mathrm{mL}$ caffeine on mRNA levels of oocytes is depicted in Figure 7-2. There was no effect of treatment or time, nor any treatment $x$ time 
interactions for any of the candidate genes (i.e. Atr, Bmp15, Cx37, and Gdf9). Atr levels appeared to decrease over time, however this was not significant $(P=0.069)$. The $R p / 19$ levels were similar across both treatment groups and exposure times.

A

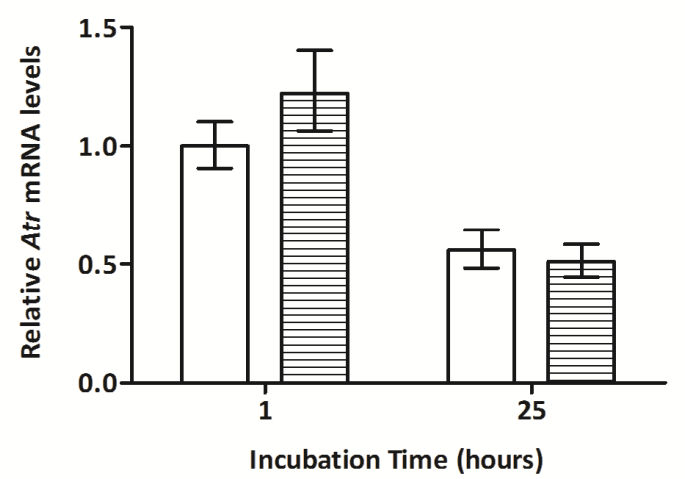

C

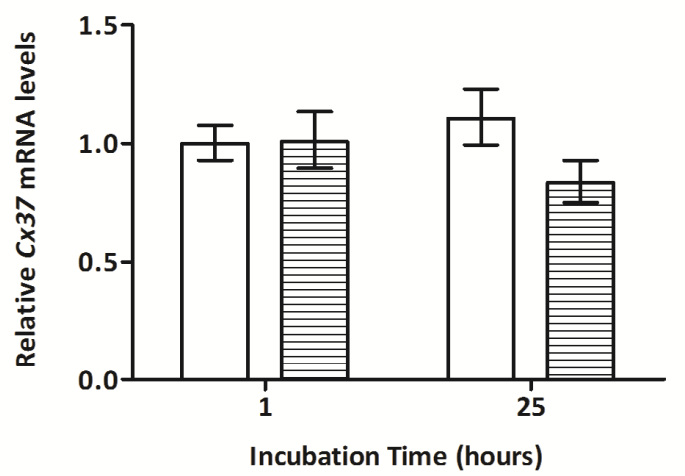

$E$

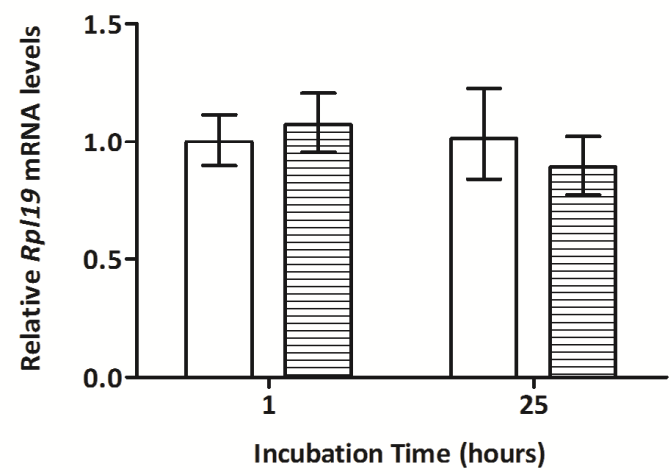

B

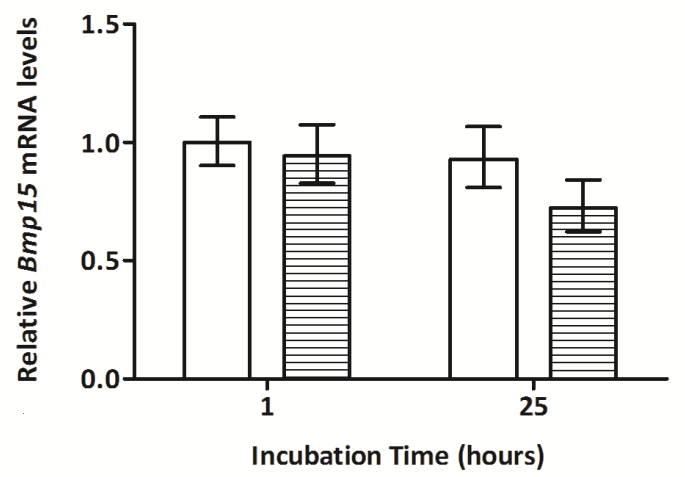

D

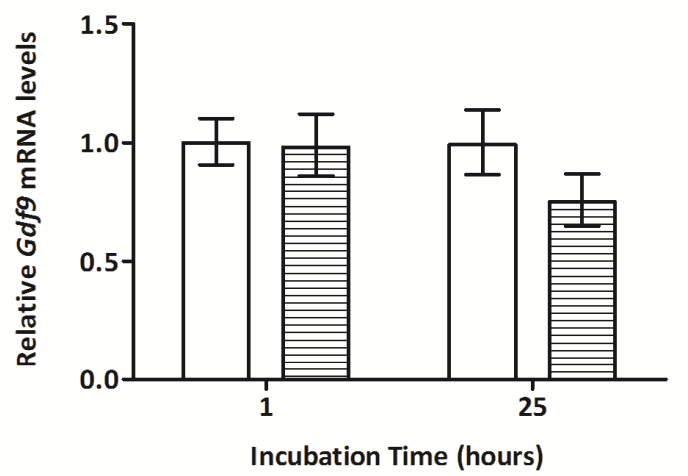

Figure 7-2: Effect of caffeine on mRNA levels of Atr (A), Bmp15 (B), Cx37 (C), Gdf9 (D), and Rpl19 (E) in rat oocytes. The control group is represented by the unfilled bars $(\square)$, while the caffeine-treated group is represented by the hatched bars ( normalised against the mean of the control group at 1 hour. The data were generated from a total of 10-12 readings from individual oocytes in each group. A two-way ANOVA was performed on untransformed data ( $B$, $C, D)$, square-root transformed data (A), or natural log transformed data (E). 


\subsubsection{Effect of ethanol on gene expression in rat oocytes}

The effect of in vitro exposure of rat COC to $7.5 \mathrm{mg} / \mathrm{mL}$ ethanol in mRNA levels of oocytes is depicted in Figure 7-3. There were no significant effects of treatment, time or treatment $x$ time interactions on Atr, Bmp15, Cx37, Gdf9, or Rp/19 mRNA levels. No significant effects of treatment or time, or treatment $\mathrm{x}$ time interactions on $R p / 19$ levels were observed. Rp/19 levels appeared to be higher in the ethanol-treated DO than the control however this was not significant $(P=0.065)$.

\subsubsection{Effect of nicotine on gene expression in rat oocytes}

The effect of in vitro exposure of rat COC to $500 \mathrm{ng} / \mathrm{mL}$ nicotine on mRNA levels of oocytes is depicted in Figure 7-4. There was no effect of treatment or time, or a treatment $x$ time interaction on Atr, Bmp15 or Gdf9 levels in rat oocytes. While not significant, Atr levels did appear to decline with time $(P=0.068)$. For $C \times 37$, there was no significant effect of treatment or time however a significant treatment $x$ time interaction was noted. Whilst $C \times 37$ mRNA levels were similar at both time points in the control oocytes, Cx37 levels appeared to decrease with time in the nicotine-treated oocytes. There was no effect of treatment or time, or a treatment $x$ time interaction on Rpl19 levels in rat oocytes.

\subsubsection{Effect of MDMA on gene expression in rat oocytes}

The effect of in vitro exposure of rat COC to $2 \mu \mathrm{g} / \mathrm{mL}$ MDMA on mRNA levels of oocytes is depicted in Figure 7-5. There were no significant effects of treatment or time, or treatment $x$ time interactions with respect to the levels of Atr, Bmp15, Cx37, Gdf9, or Rp/19 mRNA in rat oocytes.

\subsubsection{Effect of THC on gene expression in rat oocytes}

The effect of in vitro exposure of rat COC to $500 \mathrm{ng} / \mathrm{mL}$ THC on mRNA levels of oocytes is depicted in Figure 7-6. There were no significant effects of treatment or time, or treatment $x$ time interactions with respect to levels of Atr or Gdf9 mRNA in rat oocytes. For Bmp15 and Cx37, there were significant effects of time but there were no treatment or treatment $x$ time interactions: the levels for both of these genes increased with time, especially in the THCtreated groups. There were no significant effects of treatment, time, or treatment $x$ time interactions on $R p / 19$ levels in rat oocytes. 
A

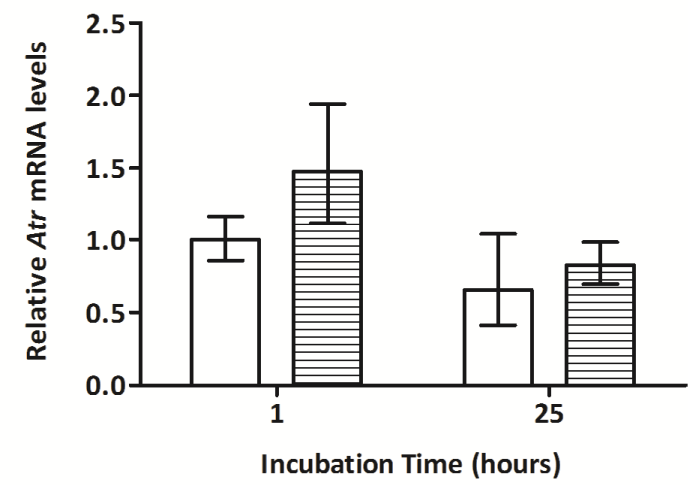

C

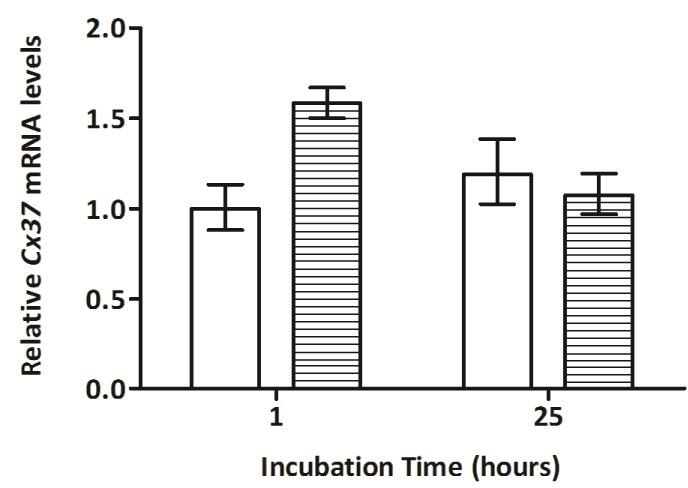

$E$

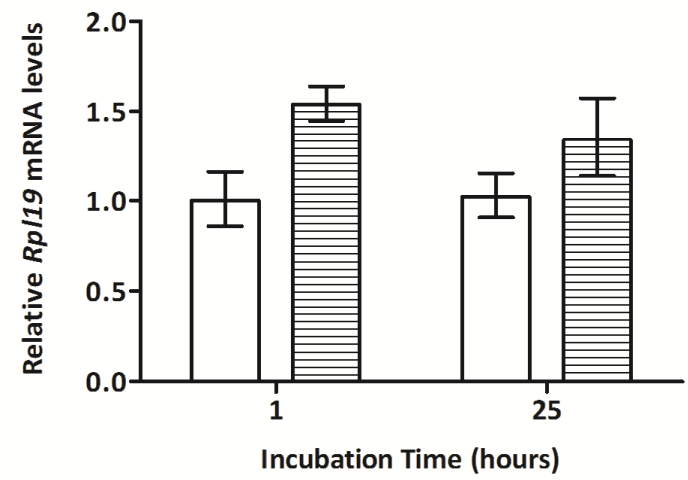

B

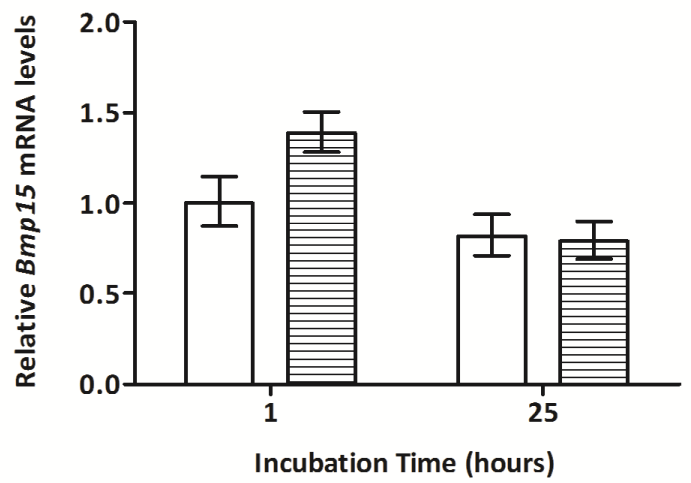

D

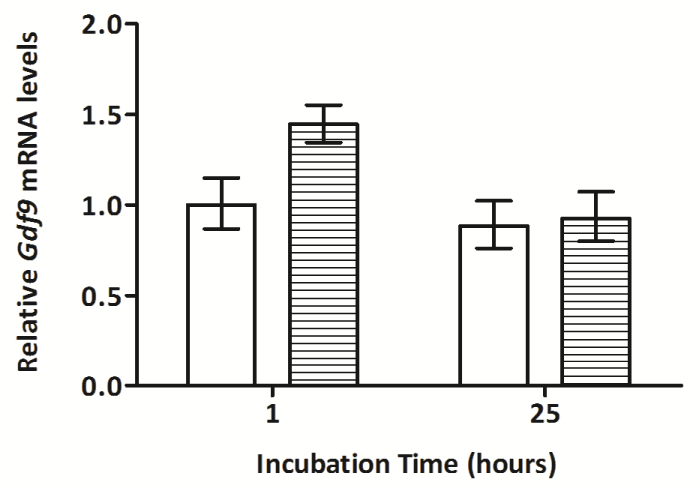

Figure 7-3: Effect of ethanol on mRNA levels of $\operatorname{Atr}(\mathrm{A}), B m p 15$ (B), Cx37 (C), Gdf9 (D), and Rpl19 (E) in rat oocytes. The control group is represented by the unfilled bars $(\square)$, while the ethanol-treated group is

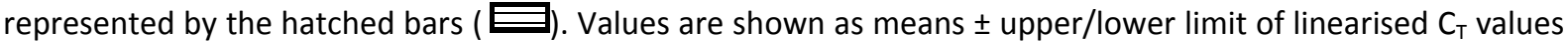
normalised against the mean of the control group at 1 hour. The data were generated from a total of 10-12 readings from individual oocytes in each group. A two-way ANOVA was performed on untransformed data ( $B$, $C, D)$, or square root transformed data $(A, E)$. 
A

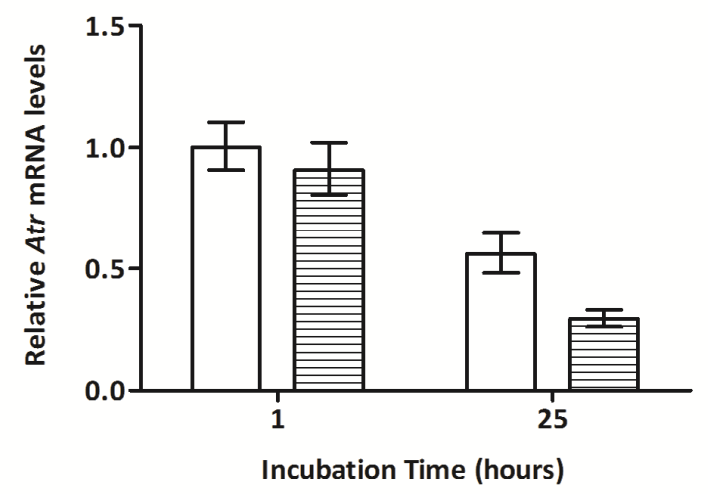

C

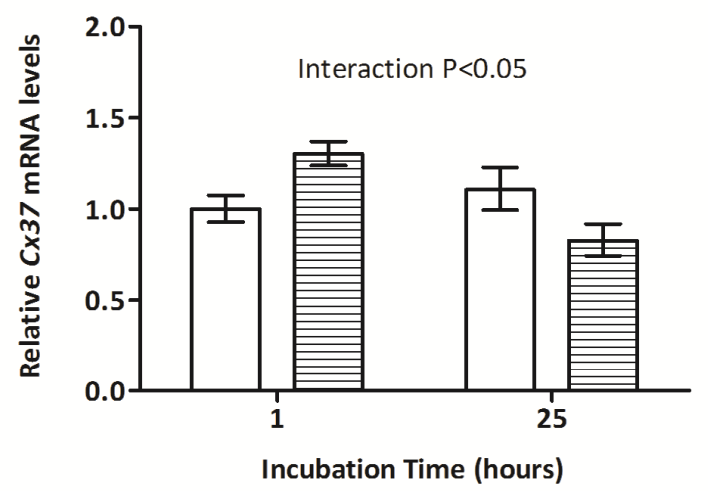

E

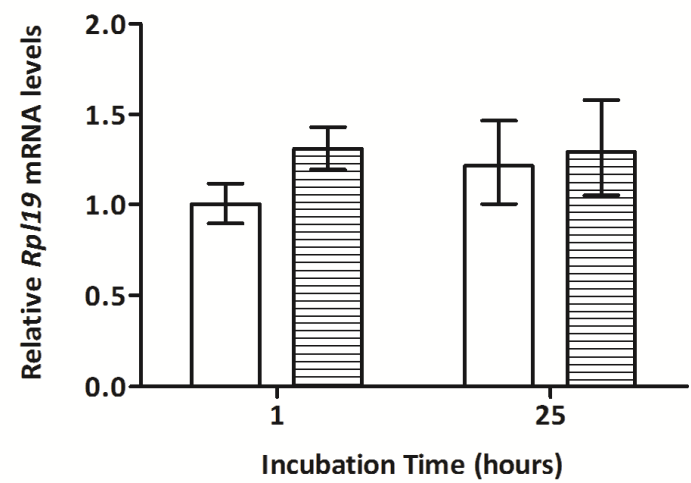

B

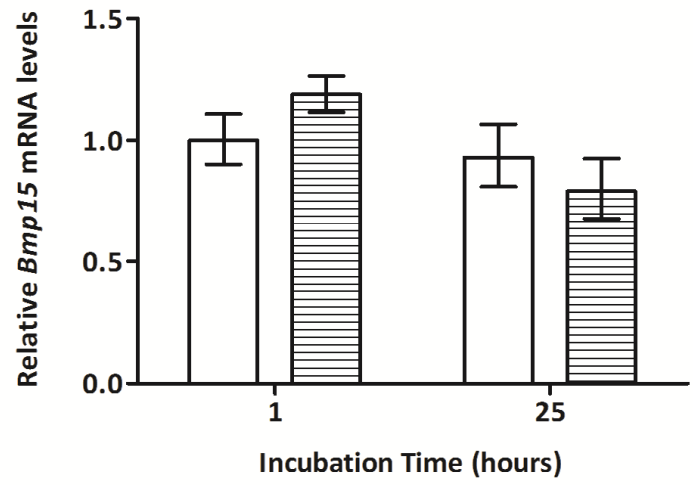

D

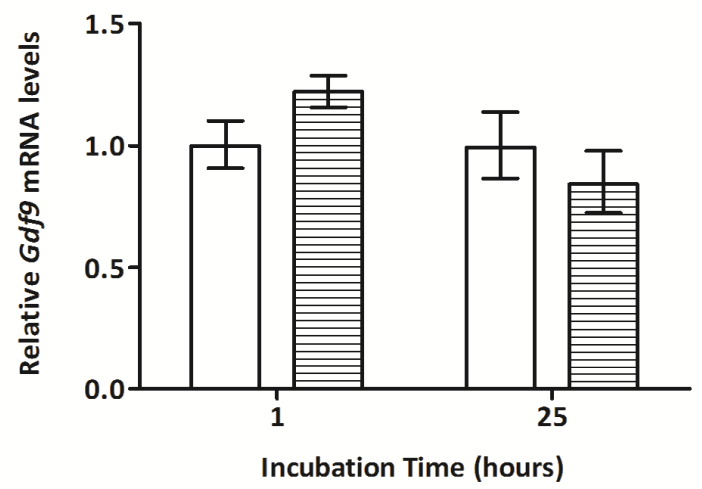

Figure 7-4: Effect of nicotine on mRNA levels of $\operatorname{Atr}(\mathrm{A}), \mathrm{Bmp15}$ (B), Cx37 (C), Gdf9 (D), and Rp/19 (E) in rat oocytes. The control group is represented by the unfilled bars ( $\square$ ), while the nicotine-treated group is represented by the hatched bars ( Values are shown as means \pm upper/lower limit of linearised $\mathrm{C}_{\mathrm{T}}$ values normalised against the mean of the control group at 1 hour. The data were generated from a total of 9-12 readings from individual oocytes in each group. A two-way ANOVA was performed on untransformed data ( $A$, $B, C, D)$ or natural log transformed data (E). 
A

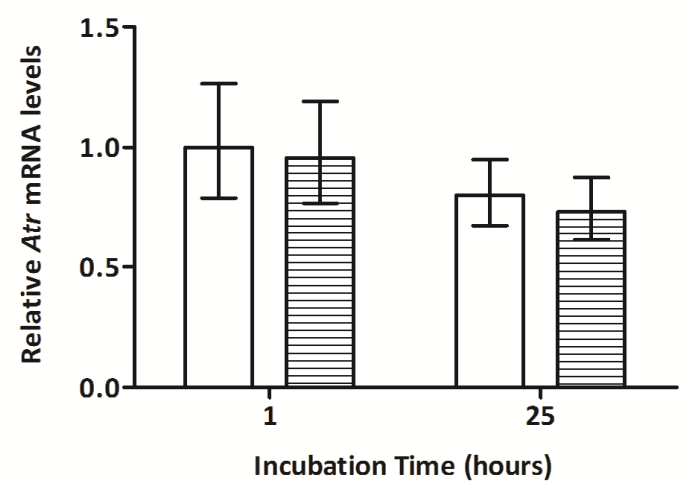

C

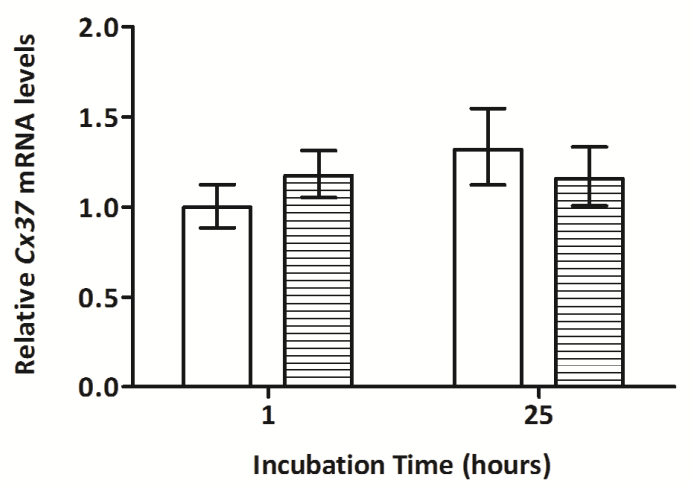

$\mathrm{E}$

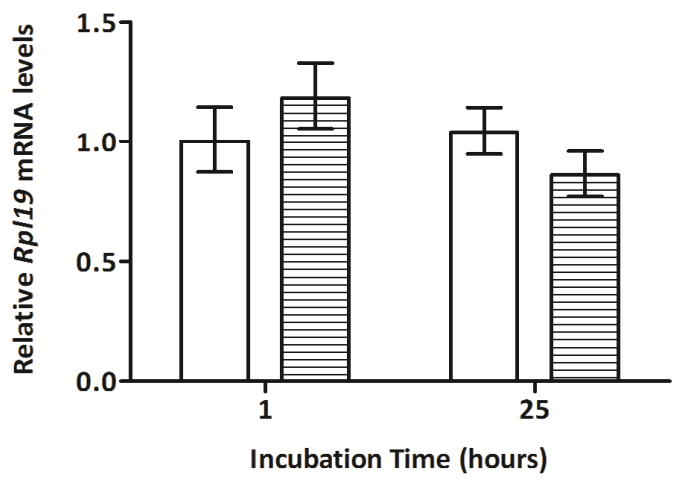

B

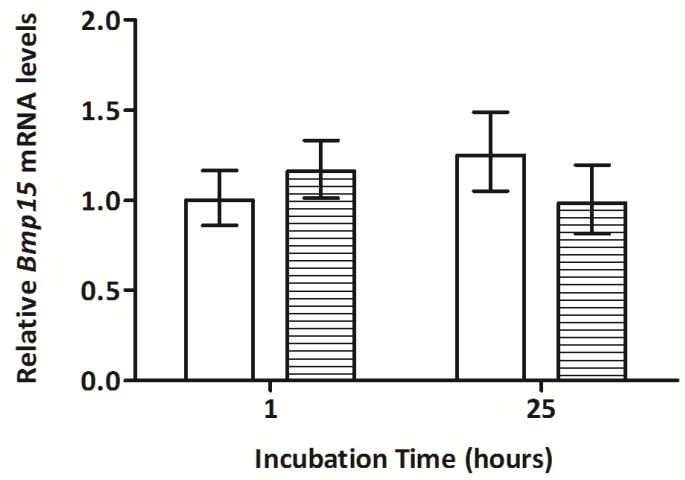

D

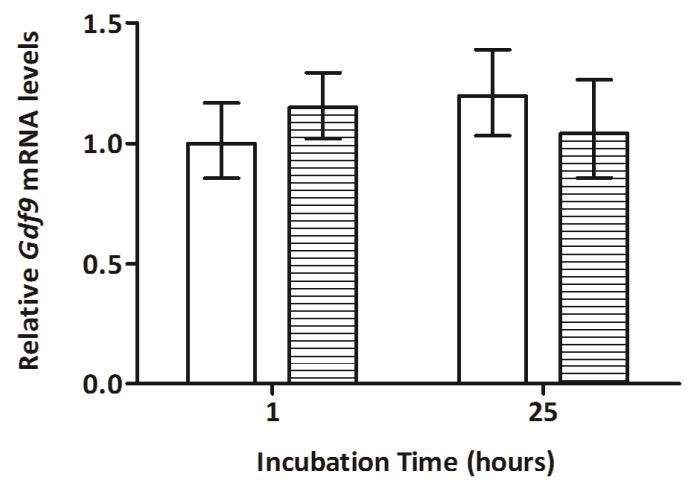

Figure 7-5: Effect of MDMA on mRNA levels of Atr (A), Bmp15 (B), Cx37 (C), Gdf9 (D), and Rpl19 (E) in rat oocytes. The control group is represented by the unfilled bars $(\square)$, while the MDMA-treated group is represented by the hatched bars ( $\overline{~(~ V a l u e s ~ a r e ~ s h o w n ~ a s ~ m e a n s ~} \pm$ upper/lower limit of linearised $C_{T}$ values normalised against the mean of the control group at 1 hour. The data were generated from a total of 10-12 readings from individual oocytes in each group. A two-way ANOVA was performed on untransformed data ( $B$, C, D, E) or square-root transformed data (A). 
A

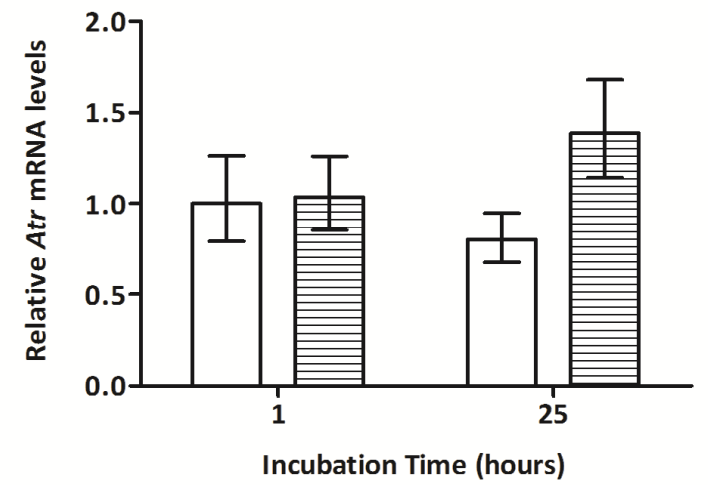

C

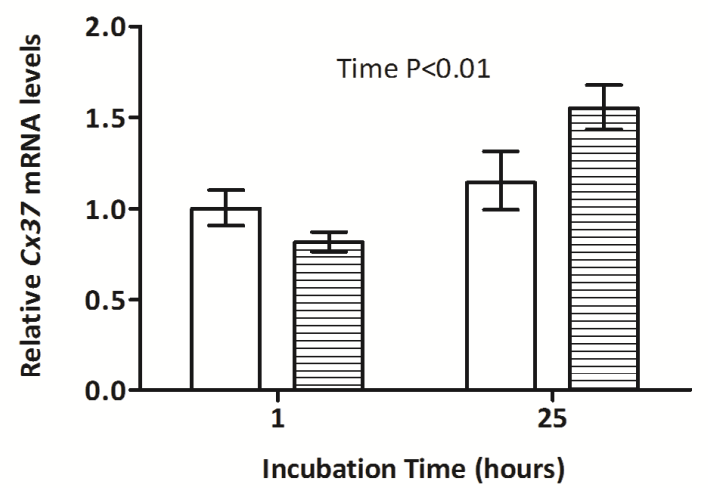

E

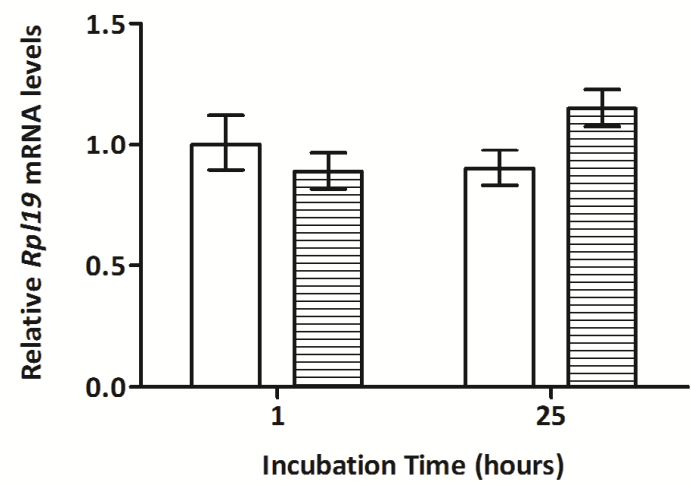

B

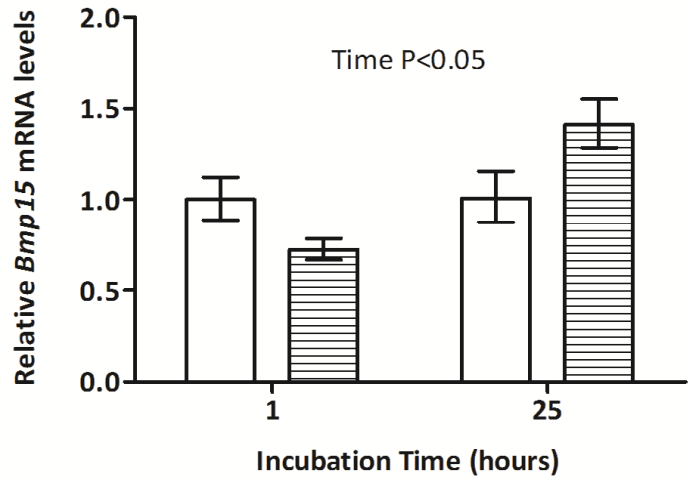

D

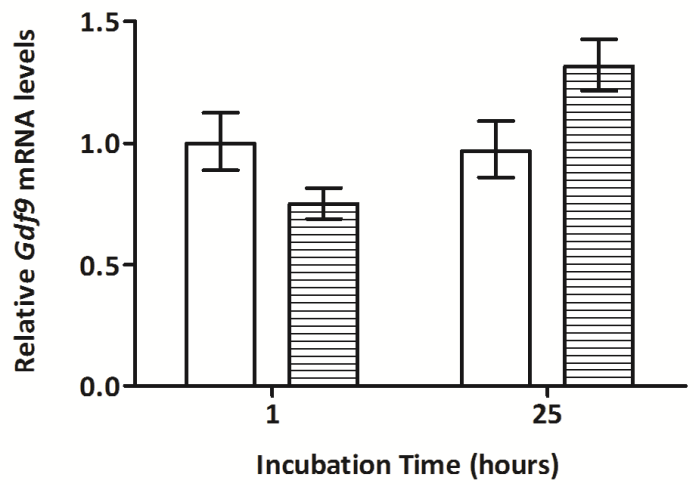

Figure 7-6: Effect of THC on mRNA levels of Atr (A), Bmp15 (B), Cx37 (C), Gdf9 (D), and Rpl19 (E) in rat oocytes. The control group is represented by the unfilled bars $(\square)$, while the THC-treated group is represented by the hatched bars ( Values are shown as means \pm upper/lower limit of linearised $\mathrm{C}_{\mathrm{T}}$ values normalised against the mean of the control group at 1 hour. The data were generated from a total of 10-12 readings from individual oocytes in each group. A two-way ANOVA was performed on untransformed $(B, C, D$, E) or natural log transformed data $(A)$. 


\subsection{Discussion}

These results reveal that the expression of the oocyte-derived genes measured in this study are mostly unchanged following exposure to a range of lifestyle factors and environmental contaminants. There was a treatment $x$ time interaction for $C \times 37$ mRNA expression following nicotine exposure, where nicotine caused a reduction in $C \times 37$ mRNA levels over time in rat oocytes, compared to an increase in control oocytes. Surprisingly, in vitro exposure to BPA, caffeine, ethanol, MDMA, or THC alone had no effect on the mRNA levels in rat oocytes of any of the genes tested.

CX37 is the primary component of oocyte-derived GJ channels (Simon et al., 1997; Teilmann, 2005) and is essential for transfer of nutrients to the oocyte. Ratchford et al. (2008) demonstrated an association between the reduced health of oocytes from diabetic mice, reduced $C \times 37$ mRNA levels, and impaired GJ coupling between the oocyte and CC. Therefore, the changes in $C_{x 3} 3$ expression levels after exposure to nicotine may be of biological significance.

The oocytes used in this study were collected following a GJ dye transfer assay. As discussed previously (Chapter 6), this GJ assay involved COC being exposed to a treatment for $0,4,12$, or 24 hours. Thereafter $\mathrm{COC}$ were incubated with calcein-AM for 10 minutes and then incubated for a further hour in calcein-free media, but still in the presence of the treatment. The results presented in this chapter suggest that nicotine raises $C \times 37$ expression in the oocyte following a 1 hour exposure, but this effect is attenuated after a 25 hour exposure. The finding that nicotine had different effects depending on exposure time may be related to the metabolism of nicotine over time. It is reasonable to expect that after a 1 hour exposure, most of the nicotine would not have been metabolised. However, after 25 hours in vitro it is possible that most of the nicotine had been metabolised. As discussed in Chapter 6, CYP2A6, which catalyses the conversion of nicotine to cotinine, is expressed in the mouse ovary (Cannady et al., 2003). Moreover, cotinine has been detected in follicular fluid (Zenzes et al., 1996; Shoaib and Stolerman, 1999).

Interestingly, exposure to BPA, caffeine, ethanol, MDMA, or THC did not affect mRNA levels of any of the oocyte-derived genes tested. This was unexpected, as BPA, caffeine, and ethanol have all been reported to directly affect oocyte quality following in vitro exposure 
(Jagiello et al., 1972; O'Neill et al., 1989; Lenie et al., 2008). However, the oocytes in the present study were cultured as intact COC. The CC may have acted as a protective barrier, perhaps preventing at least a proportion of the harmful factors from direct action on the oocyte. In addition, the variability that exists between the maturation stages of individual oocytes may have masked any treatment effects.

The levels of oocyte-derived Rp/19 were not affected by any of the treatments tested in this study. Similarly, levels of Rp/19 were not affected by incubation time with the notable exception of the BPA experiment. Levels of Rp/19 decreased over time in the BPA experiment, indicating that oocytes containing less total RNA were selected at the 25 hour time point. Expression levels of many of the candidate genes showed a similar pattern to that of Rp/19. Overall these results reveal that expression levels of oocyte-derived Atr, Bmp15, Cx37, and Gdf9 are generally stable regardless of time that the COC spent in culture.

In summary, the major finding from this chapter was that exposure of rat COC to potentially detrimental compounds (i.e. BPA, caffeine, ethanol, MDMA, or THC) did not alter the overall expression of oocyte genes measured in this study. The exposure of nicotine did result in increased oocyte-derived $C_{x} 37$ at 1 hour, but decreased $C_{x} 37$ at 25 hours. The changes in Cx37 expression levels following exposure to nicotine may influence the availability of nutrients and other molecules necessary for oocyte health. 


\section{EFFECTS OF IN VITRO EXPOSURE OF COC TO LIFESTYLE FACTORS ON GENE EXPRESSION IN CC}

\subsection{Introduction}

During follicular development, the CC provide the oocyte with glucose metabolites, cholesterol metabolites, CAMP, ions and amino acids. Thus, the $\mathrm{CC}$ have a nurturing role during oocyte maturation. This is facilitated by the passing of these low molecular weight compounds to the oocyte through GJ (Kidder and Vanderhyden, 2010). However, in addition to important substrates for oocyte maturation, the follicular fluid microenvironment contains exudates from the plasma so that the $\mathrm{COC}$ can also be exposed to a number of potentially harmful environmental or lifestyle factors (Weiss and Eckert, 1989; Zenzes et al., 1996; Al-Saleh et al., 2010).

As the CC surround the oocyte within the follicle, they are also considered to have a protective role for the oocyte. For example, the CC protect the oocyte from oxidative stress during follicular development (Tatemoto et al., 2000). Exposure to a reactive oxidative species generating system (XOD) caused meiotic arrest in DO, and this was coincident with the activation of caspase- 3 and thus increased activation of the apoptotic pathway. In contrast, COC that were exposed to XOD did not exhibit this activation of caspase-3 and had higher levels of the antioxidant glutathione (Tatemoto et al., 2000).

In Chapter 6 it was determined that in vitro exposure of COC to BPA or nicotine resulted in increased GJ transfer from the CC to the oocyte. However, in vitro exposure of $\mathrm{COC}$ to caffeine, ethanol, MDMA, or THC did not affect GJ communication. In Chapter 7, it was found that an interaction between time and nicotine treatment was observed for the oocyte GJ gene, $C \times 37$. The effects of these compounds on gene expression in oocytes are most likely facilitated through regulatory mechanisms within CC. Thus, these compounds would be expected to cause even larger perturbations in gene expression levels of CC.

The aim of this chapter was to determine the effects of in vitro exposure of rat $\mathrm{COC}$ to BPA, caffeine, ethanol, nicotine, MDMA, or THC on Atr, Cx43, Cycs, Gfpt1, and Pfkp mRNA expression levels in the CC. 


\subsection{Methods}

The collection of CC masses from individual $\mathrm{COC}$ was undertaken following completion of the GJ bioassays described in Chapter 6 . The samples were stored at $-80{ }^{\circ} \mathrm{C}$ prior to total RNA extraction as described in Section 2.3.1. The incubation times referred to henceforth indicate the total time each $\mathrm{COC}$ was incubated with the treatment in Chapter 6 (i.e. a 0 or 24 hour pre-calcein incubation followed by a 1 hour post-calcein incubation). The mRNA levels of the candidate genes were measured in quadriplex reaction (Atr/Cycs/Glut1 and Cx43/Gfpt1/Pfkp, respectively) using multiplex TaqMan qPCR as previously described (Section 2.3.2). Glut1 was excluded from the final analysis, as expression levels were below the detection limit. The final gene in each multiplex reaction was the reference gene $R p / 19$, which was used to correct gene expression for RNA input. Each reaction run included a calibrator sample which contained CDNA from a pool of rat COC; this allowed comparisons to be made between reaction runs. The mRNA levels for each candidate gene were corrected for the Rp/19 levels of that sample. Subsequently, the data were analysed using the $2^{(-\Delta \Delta C T)}$ method (Livak and Schmittgen, 2001).

The relative linear mRNA levels were analysed for normality using the D'Agostino \& Pearson normality test. Data that were not normally-distributed were transformed (using natural log, square root, square $\operatorname{root}(y+0.5), y / y+1$, or squared transformations) and reanalysed to confirm a normal distribution. The normally-distributed data were analysed by a two-way ANOVA. 


\subsection{Results}

\subsubsection{Effect of BPA on gene expression in rat CC}

The effect of in vitro exposure of COC to $20 \mathrm{ng} / \mathrm{mL}$ BPA on mRNA levels in CC is depicted in Figure 8-1. There were no effects of time or treatment, or a treatment $x$ time interaction on Atr, Gfpt1, and Pfkp mRNA levels: the apparent decline in Pfkp levels with time in culture in the control group was not significant $(P=0.068)$ However, there was a treatment $x$ time interaction for $C_{x} 43$ mRNA levels, along with treatment and time main effects. The expression levels for $\mathrm{C}_{X} 43$ remained steady over time in the control $\mathrm{CC}$, but had increased markedly after 25 hours in culture in the BPA-treated CC. There was a significant decline in Cycs mRNA levels over time in both the control and BPA-treated CC. There were no effects of time, treatment, or a treatment $\mathrm{x}$ time interaction on Rp/19 mRNA levels.

\subsubsection{Effect of caffeine on gene expression in rat CC}

The effect of in vitro exposure of COC to $100 \mu \mathrm{g} / \mathrm{mL}$ caffeine on mRNA levels in rat CC is depicted in Figure 8-2. There were no significant effects of treatment, time, or a treatment $x$ time interaction on Atr mRNA levels although the mean expression levels were $~ 50 \%$ lower $(P=0.069)$ at 25 hours in both the control and caffeine-treated CC. Both treatment and time effects were measured for $C_{X} 43$ mRNA where levels were reduced at 25 hours, compared to 1 hour, in both control and caffeine-treated CC. Moreover, $C \times 43$ mRNA was further reduced in the caffeine-treated CC, compared to control CC at both time points. There was an effect due to time, but not treatment, or a treatment $x$ time interaction for Cycs, Gfpt1, and Pfkp mRNA levels. Expression levels of all of these genes were reduced at 25 hours, compared to 1 hour, in both the control and caffeine-treated groups. There were no effects of time, treatment, or a treatment $\mathrm{x}$ time interaction on Rp/19 mRNA levels. 
A

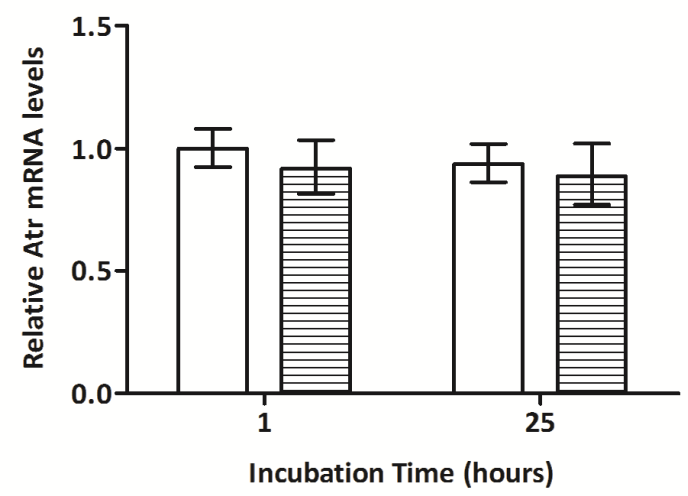

C

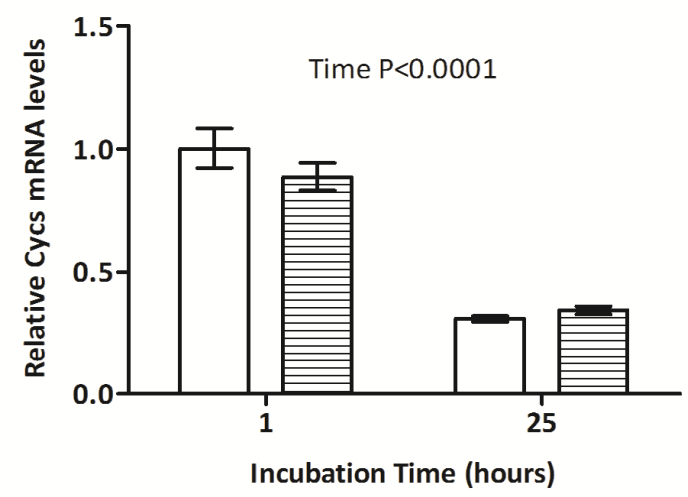

$E$

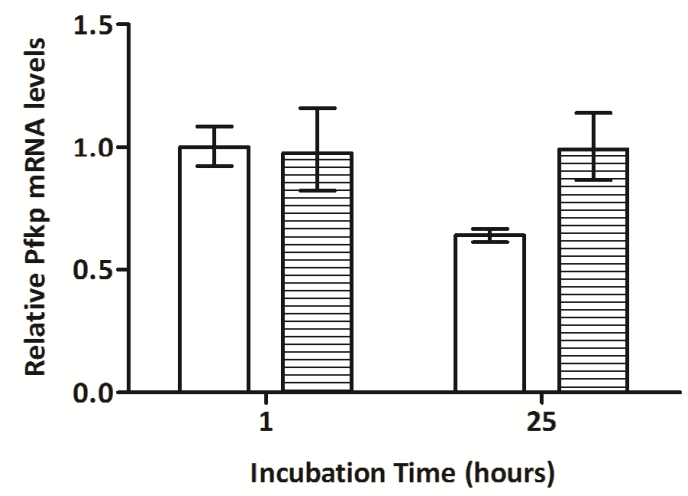

B

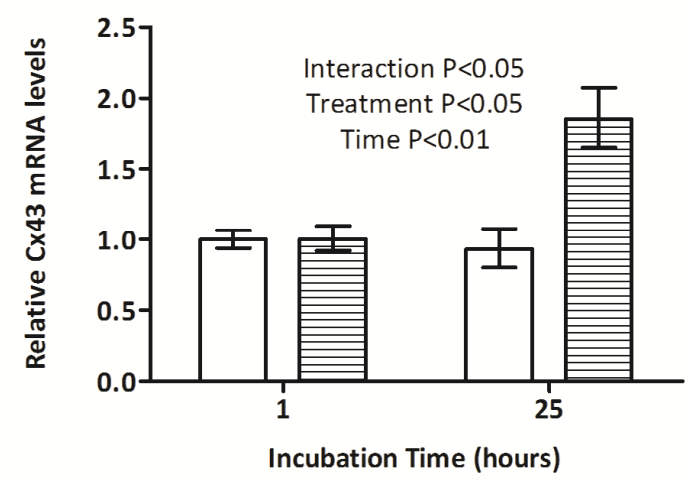

D

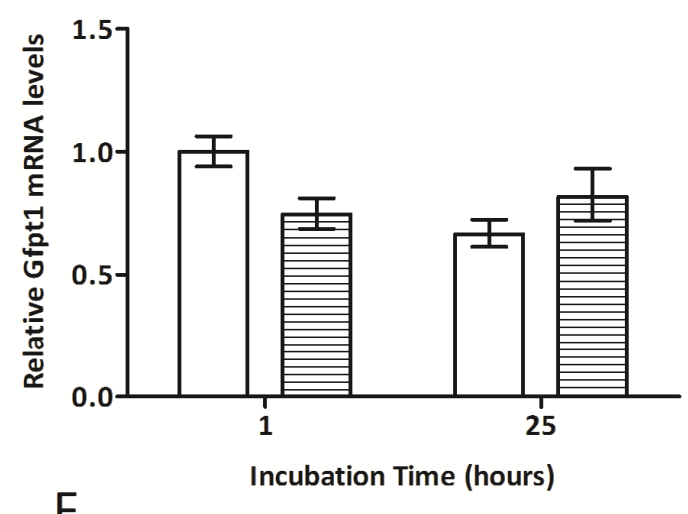

$\mathrm{F}$

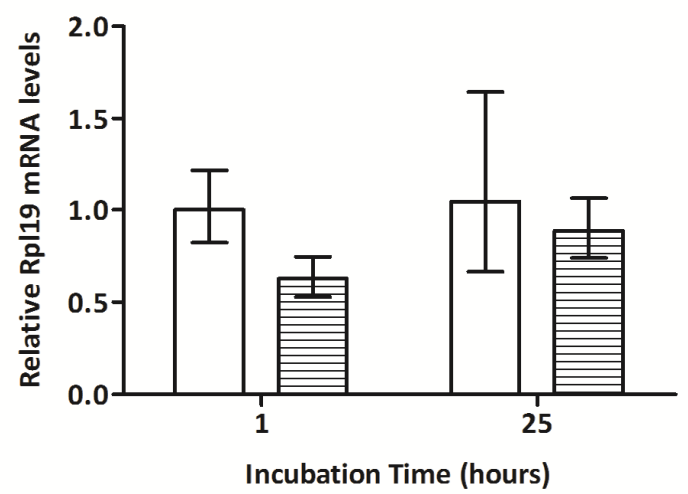

Figure 8-1: Effect of BPA on mRNA levels of Atr (A), Cx43 (B), Cycs (C), Gfpt1 (D), Pfkp (E), and Rpl19 (F) in rat CC. The control group is represented by the unfilled bars ( $\square)$, while the BPA-treated group is represented by the hatched bars ( against the mean of the control group at 1 hour. The data were generated from a total of 11-12 readings from individual oocytes in each group. A two-way ANOVA was performed on untransformed data (B, E), natural log transformed data $(D, F)$ or squared-root transformed data $(A)$, square-root $(y+0.5)$ transformed data (C). 
A

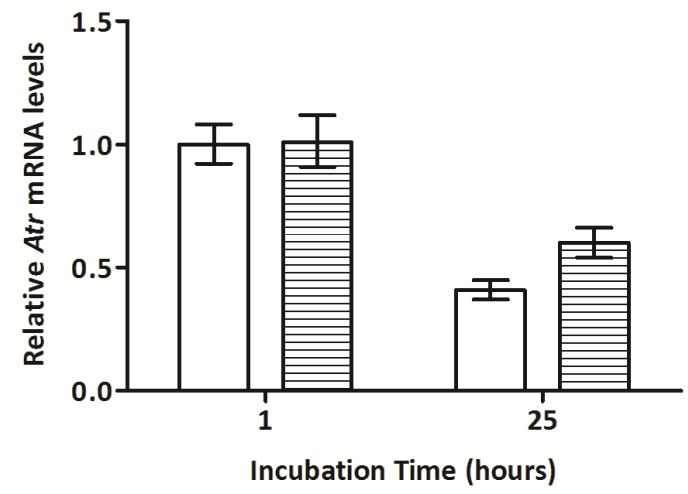

C

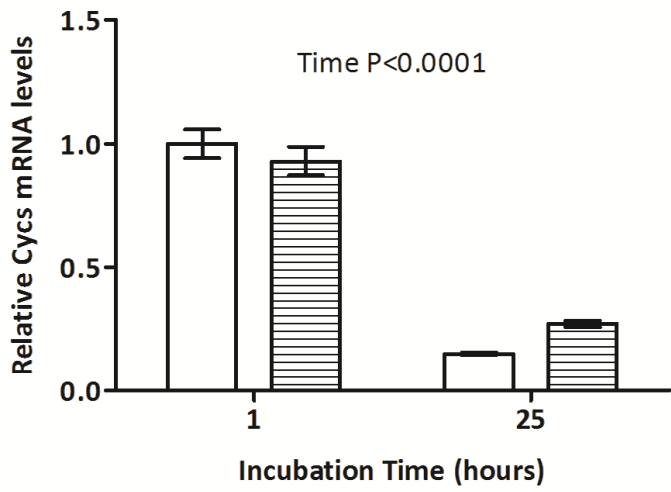

$\mathrm{E}$

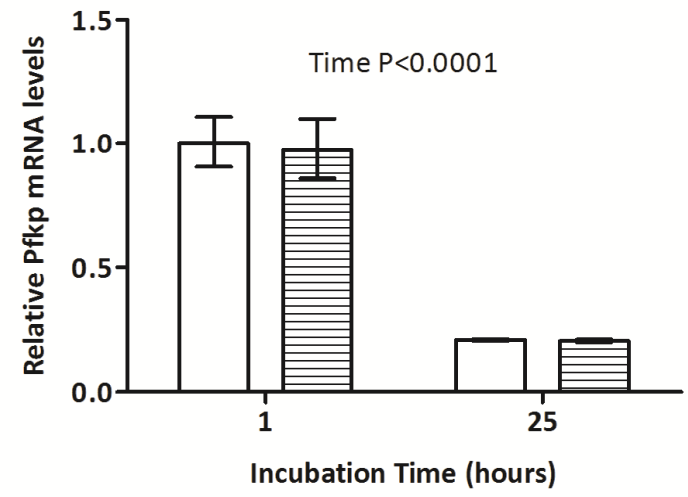

B

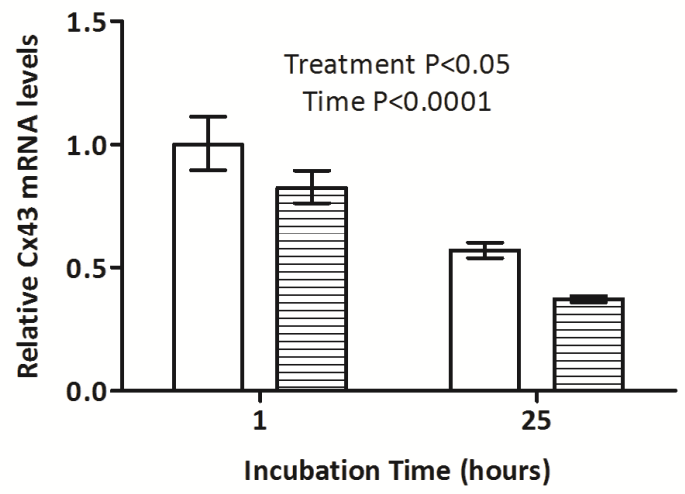

D

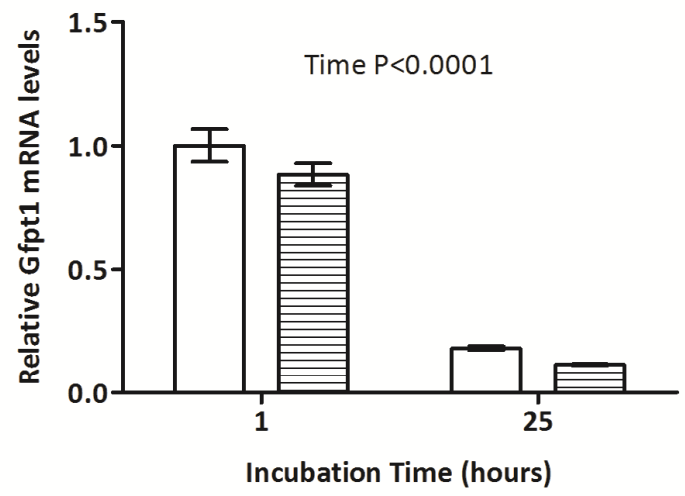

$\mathrm{F}$

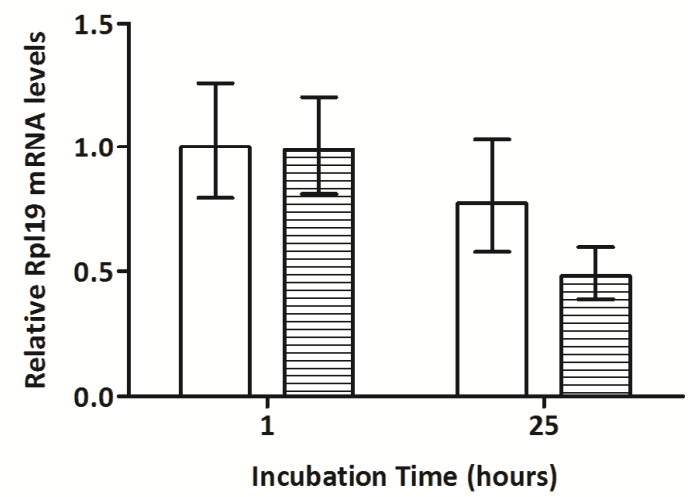

Figure 8-2: Effect of caffeine on mRNA levels of Atr (A), Cx43 (B), Cycs (C), Gfpt1 (D), Pfkp (E), and Rpl19 (F) in rat CC. The control group is represented by the unfilled bars $(\square)$, while the caffeine-treated group is represented by the hatched bars ( normalised against the mean of the control group at 1 hour. The data were generated from a total of 10-12 readings from individual oocytes in each group. A two-way ANOVA was performed on untransformed data $(A$, $C, D, E)$, natural log transformed data (B, F). 


\subsubsection{Effect of ethanol on gene expression in rat CC}

The effect of in vitro exposure of COC to $7.5 \mathrm{mg} / \mathrm{mL}$ ethanol on mRNA levels in rat CC is depicted in Figure 8-3. There were no effects of time, treatment, or a treatment $x$ time interaction on Atr mRNA levels. A treatment $x$ time interaction was observed for $C_{x} 43$ expression levels: this was likely due to the mean $C_{x} 43$ levels in ethanol-treated CC being higher than the controls at 1 hour, but similar at 25 hours. An effect of time was observed for Cycs, Gfpt1, and Pfkp mRNA levels where expression levels of all these genes were lower at 25 hours relative to 1 hour in both groups. Whilst expression levels of Gfpt1 appeared to be further reduced in the ethanol-treated, compared to the control, $\mathrm{CC}$, this did not reach significance $(\mathrm{P}=0.14)$. The mean levels of Gfpt1 expression in the ethanol-treated groups were skewed by one outlier at 1 hour (relative Gfpt1 level of 0.012) and two outliers at 25 hours (relative Gfpt1 levels of 0.004 and 0.008). However, these did not appear to affect the statistical analysis. These samples were not removed from the analysis as the $R p / 19 C_{T}$ value was within the sensitive range. There were no effects of time, treatment, or a treatment $x$ time interaction on Rp/19 mRNA levels.

\subsubsection{Effect of nicotine on gene expression in rat CC}

The effect of in vitro exposure of COC to $500 \mathrm{ng} / \mathrm{mL}$ nicotine on mRNA levels in rat CC is depicted in Figure 8-4. There were no effects of time, treatment, or a treatment $x$ time interaction on Atr mRNA levels. Although levels of Atr mRNA were lower in control CC at 25 hours compared to that at 1 hour this was not significant $(P=0.17)$. There were effects of time on $C x 43, C y c s, G f p t 1$ and Pfkp mRNA levels. Mean expression levels of each gene were reduced at 25 hours, compared to 1 hour, in both the control and nicotine-treated groups. Moreover, $C_{X} 43$ mRNA was further reduced $(P=0.052)$ in the nicotine-treated group, compared to control group at 25 hours. Conversely, the levels of Cycs mRNA were higher although not significantly so $(\mathrm{P}=0.07)$, in the nicotine-treated group compared to the control at 25 hours. There were no effects of time, treatment, or a treatment $x$ time interaction on Rpl19 mRNA levels. 
A

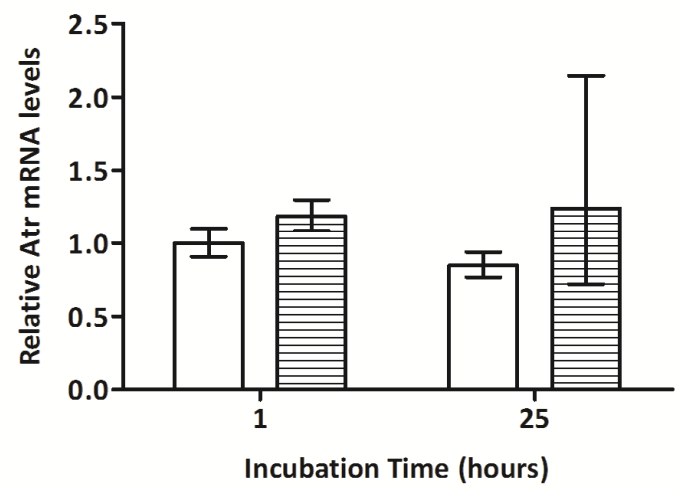

$\mathrm{C}$

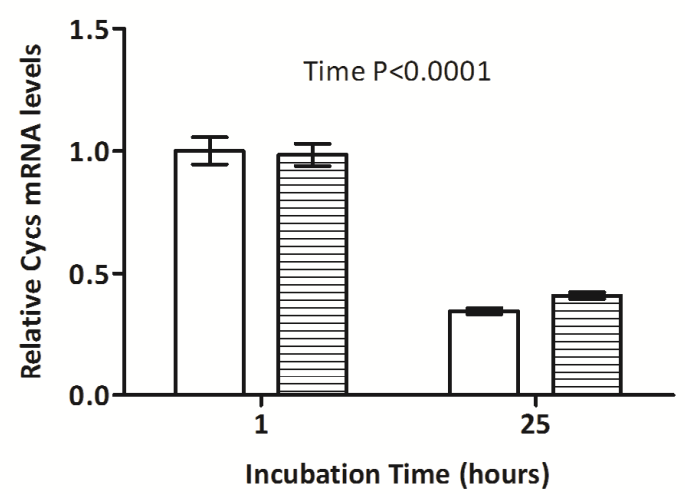

$E$

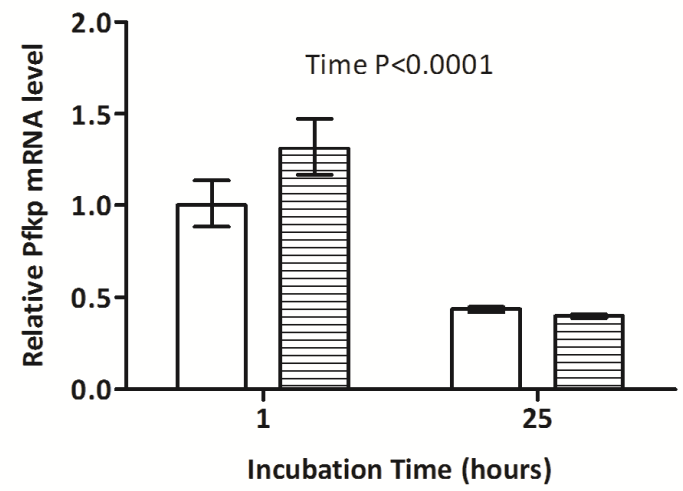

B

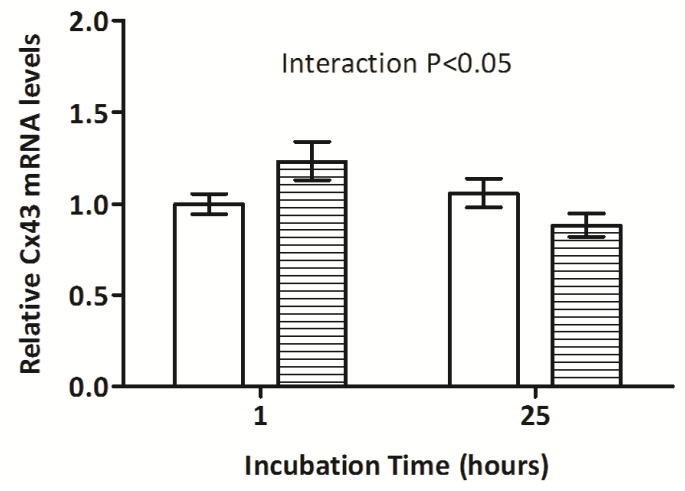

D

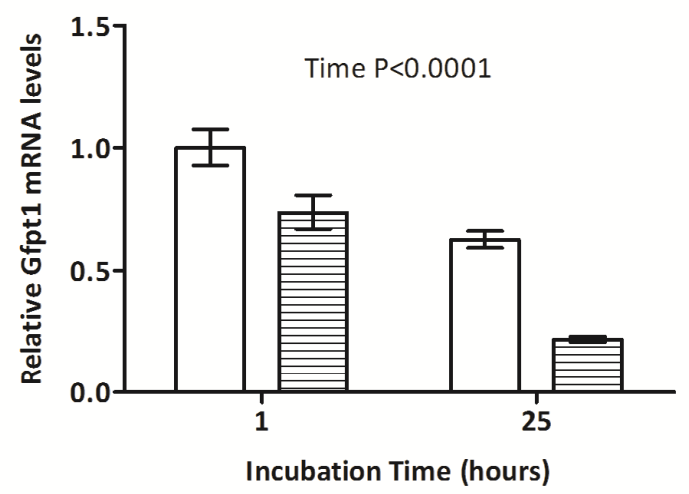

$\mathrm{F}$

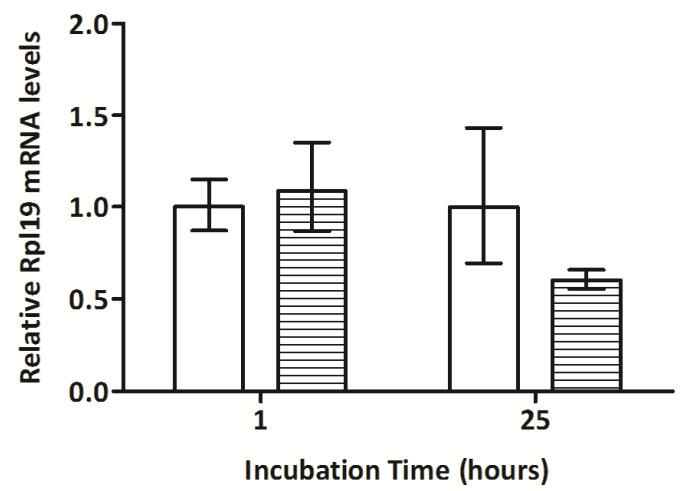

Figure 8-3: Effect of ethanol on mRNA levels of Atr (A), Cx43 (B), Cycs (C), Gfpt1 (D), Pfkp (E), and Rpl19 (F) in rat CC. The control group is represented by the unfilled bars $(\square)$, while the ethanol-treated group is represented by the hatched bars ( normalised against the mean of the control group at 1 hour. The data were generated from a total of 10-12 readings from individual oocytes in each group. A two-way ANOVA was performed on untransformed data ( $B$, $C, D, E)$, natural log transformed data $(F)$ and $1 /(y+1)$ transformed data $(A)$. 
A

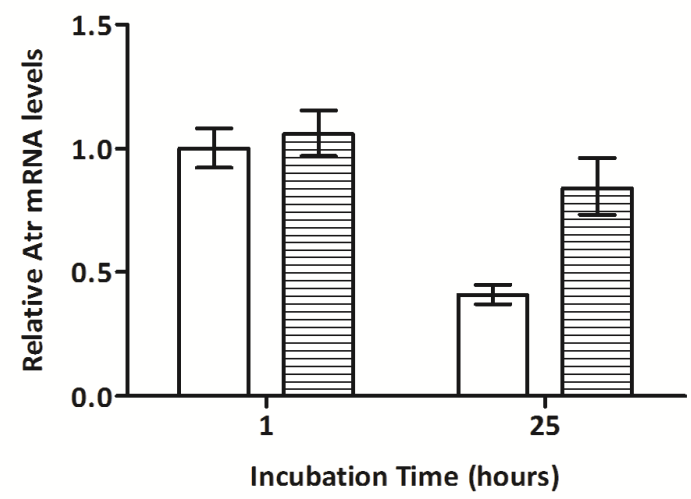

C

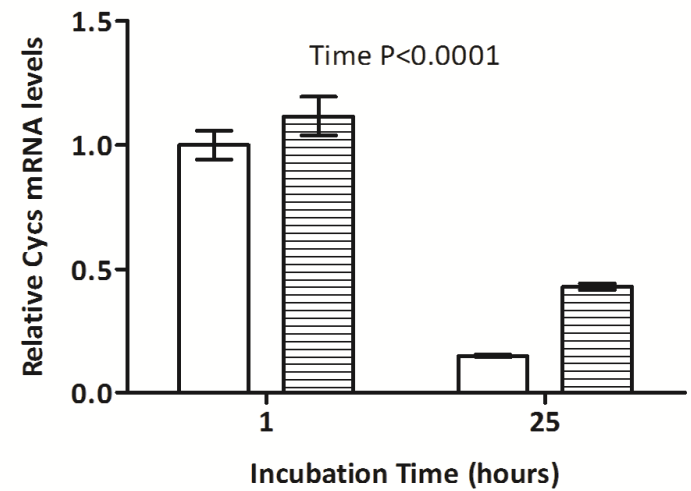

$E$

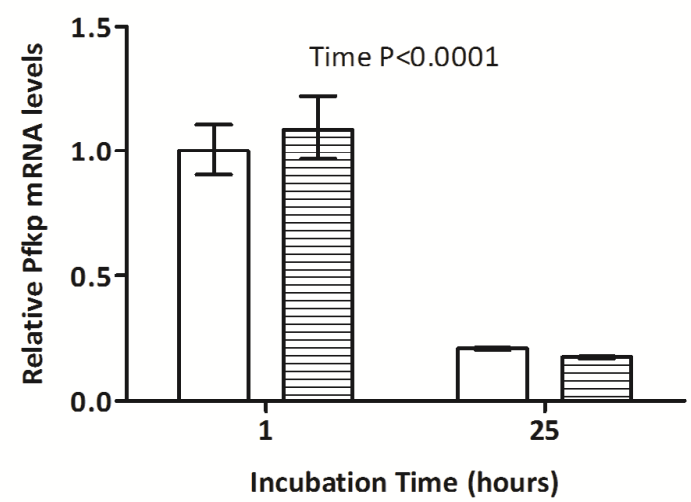

B

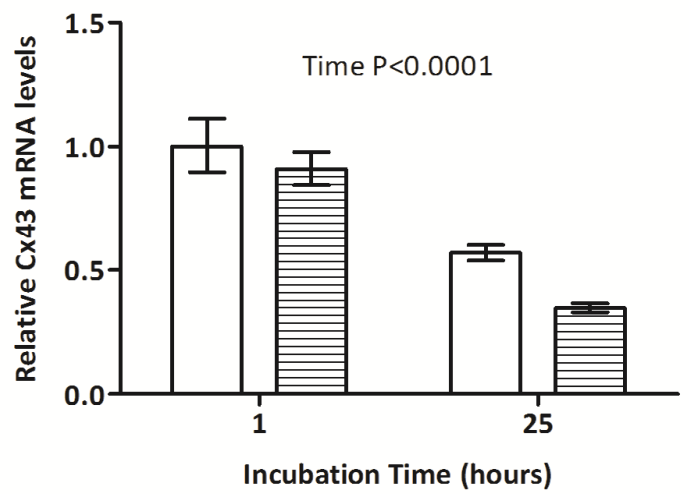

D

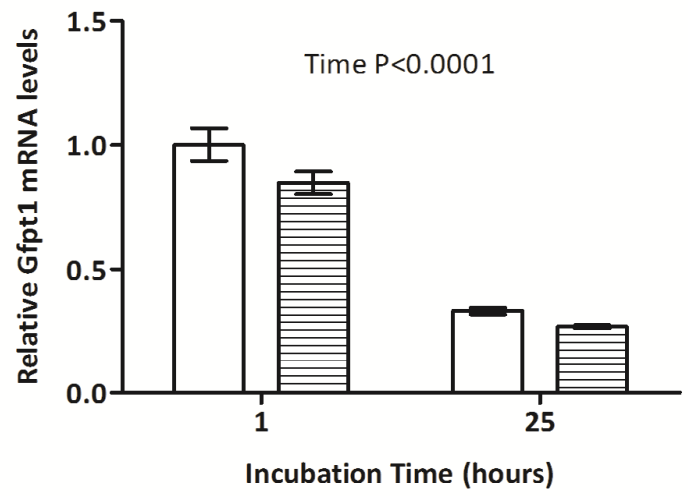

$\mathrm{F}$

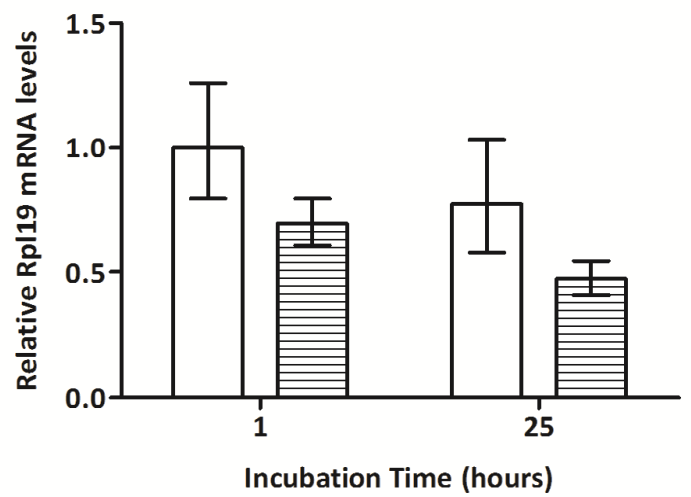

Figure 8-4: Effect of nicotine on mRNA levels of Atr (A), Cx43 (B), Cycs (C), Gfpt1 (D), Pfkp (E), and Rpl19 (F) in rat CC. The control group is represented by the unfilled bars $(\square)$, while the nicotine-treated group is represented by the hatched bars ( $\bar{~}$. Values are shown as means \pm upper/lower limit of linearised $C_{T}$ values normalised against the mean of the control group at 1 hour. The data were generated from a total of 11-12 readings from individual oocytes in each group. A two-way ANOVA was performed on untransformed data ( $A$, $C, D)$, natural log transformed data (B, E, F). 


\subsubsection{Effect of MDMA on gene expression in rat CC}

The effect of in vitro exposure of $\mathrm{COC}$ to $2 \mu \mathrm{g} / \mathrm{mL}$ MDMA on mRNA levels in rat CC is depicted in Figure 8-5. A time effect was observed for Atr mRNA whereby expression levels had increased in both groups at 25 hours, compared to 1 hour. There were treatment effects on expression levels of both $C_{x} 43$ and Gfpt1. Expression levels of $C_{x} 43$ and Gfpt1 levels were increased in the MDMA-treated group, compared to the control, at both time points. There were effects of time $\mathrm{x}$ treatment interactions for Cycs and Pfkp expression. The mean Cycs levels in the MDMA-treated group were similar at 1 hour, but reduced at 25 hours, relative to the control. Pfkp levels in the MDMA-treated group were increased at 1 hour, but were reduced at 25 hours, relative to the control group. There were no effects of time or treatment on Rp/19 mRNA levels.

\subsubsection{Effect of THC on gene expression in rat CC}

The effect of in vitro exposure of COC to $500 \mathrm{ng} / \mathrm{mL}$ THC on mRNA levels in rat CC is depicted in Figure 8-6. There was an effect of time on Atr expression whereby levels were increased in both THC-treated and control groups at 25 hours, compared to that at 1 hour. The expression levels of $C_{x} 43$ were affected by treatment and time. The mean levels of $C_{x} 43$ mRNA were similar between the two time points in the control group, but had increased with time in the THC-treated group. An effect of time was observed for both Cycs and Pfkp genes where expression levels of both Cycs and Pfkp were decreased in the control and THCtreated groups at 25 hours, compared to 1 hour. Additionally, Gfpt1 mRNA levels were increased in the THC-treated group at both time points, compared to the control. There were no effects of time or treatment on Rp/19 mRNA levels. 
A

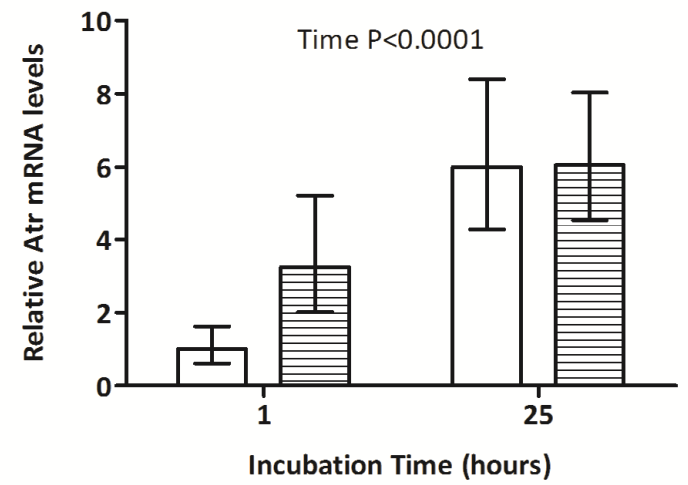

$\mathrm{C}$

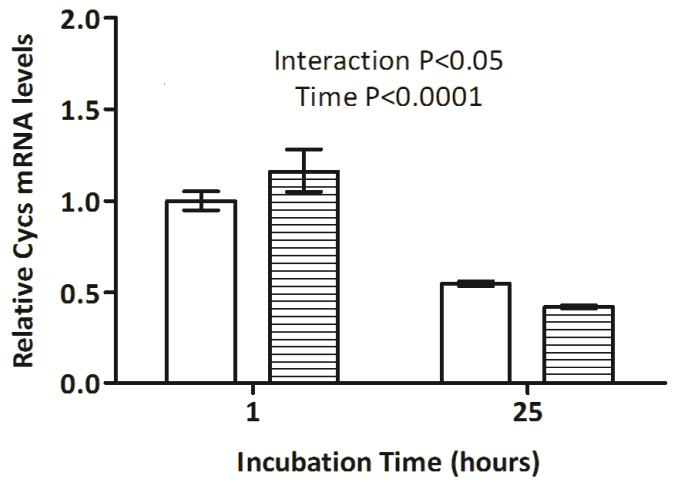

$E$

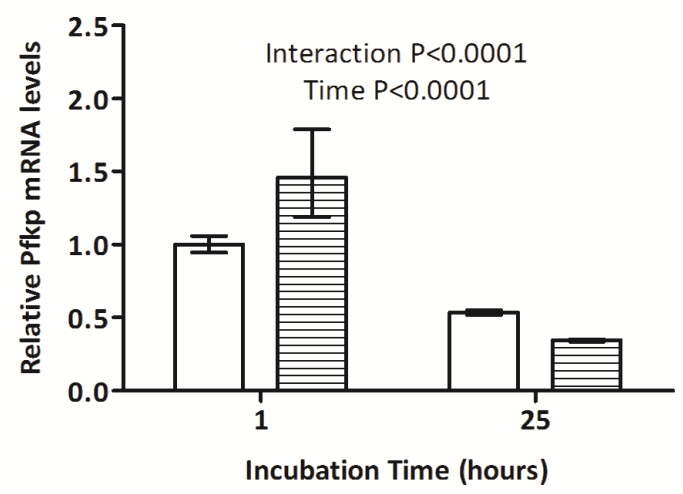

B

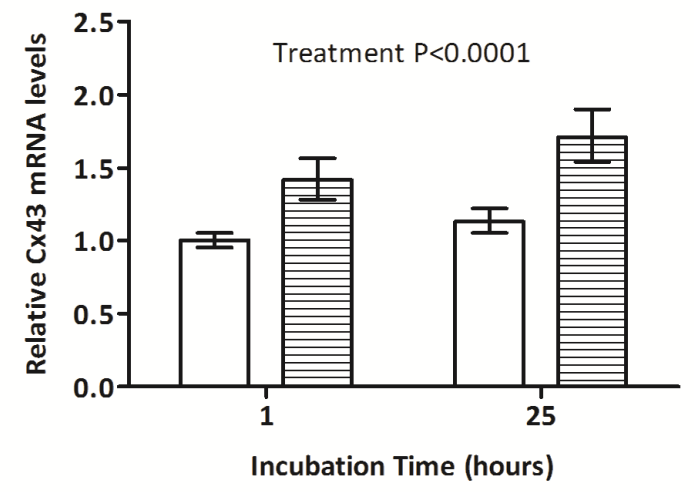

D

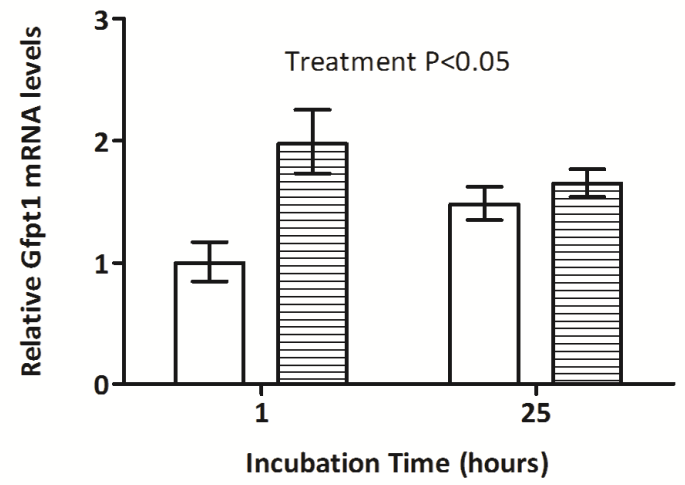

$\mathrm{F}$

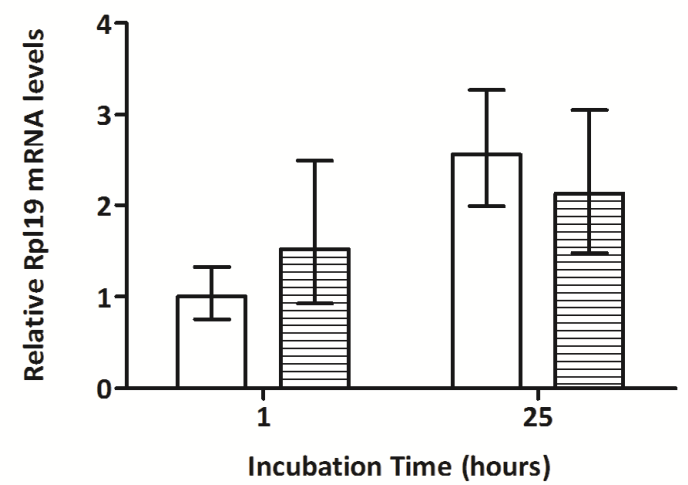

Figure 8-5: Effect of MDMA on mRNA levels of Atr (A), Cx43 (B), Cycs (C), Gfpt1 (D), Pfkp (E), and Rpl19 (F) in rat CC. The control group is represented by the unfilled bars ( $\square$ ), while the MDMA-treated group is represented by the hatched bars ( normalised against the mean of the control group at 1 hour. The data were generated from a total of 12 readings from individual oocytes in each group. A two-way ANOVA was performed on untransformed data (A, $B, C, D, F)$, natural log transformed data (E). 
A

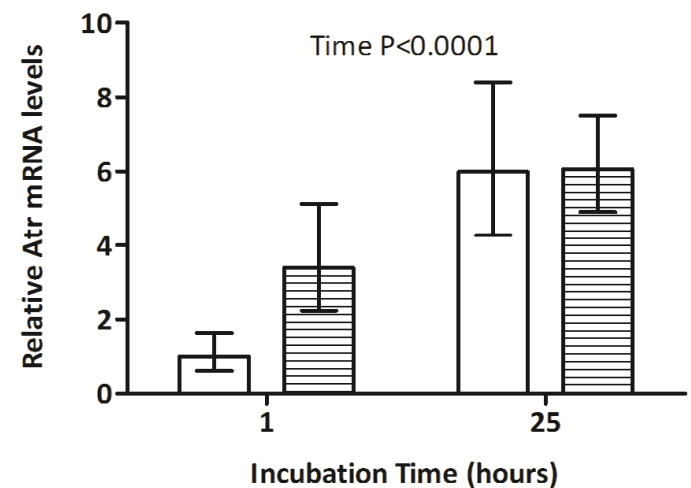

C

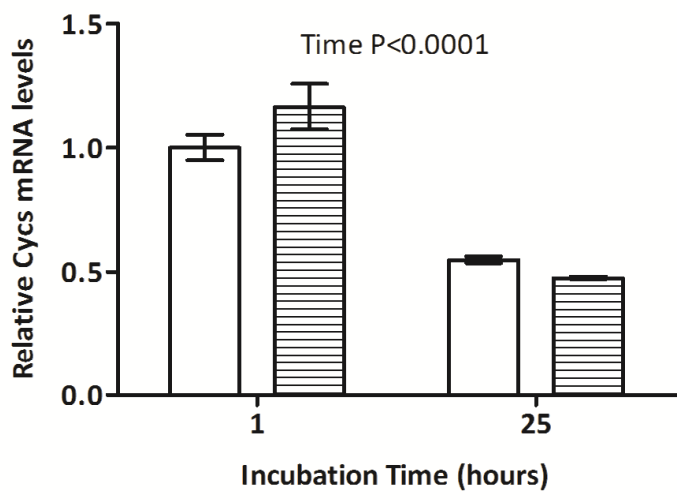

$E$

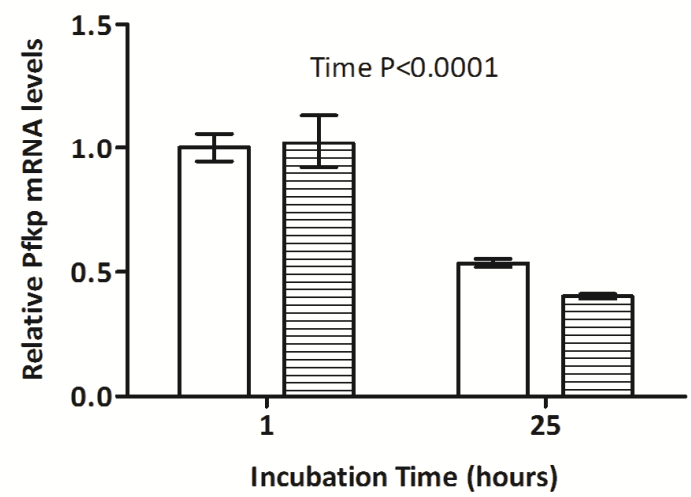

B

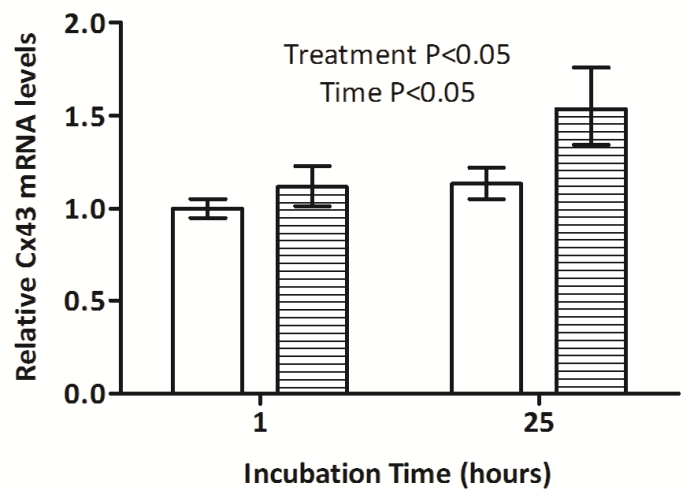

D

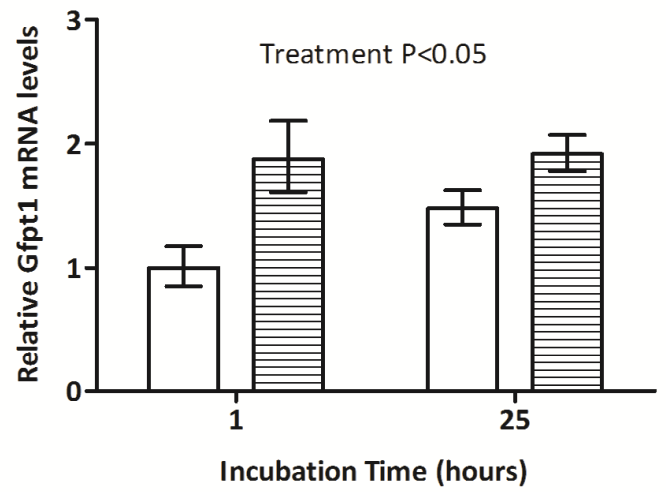

$\mathrm{F}$

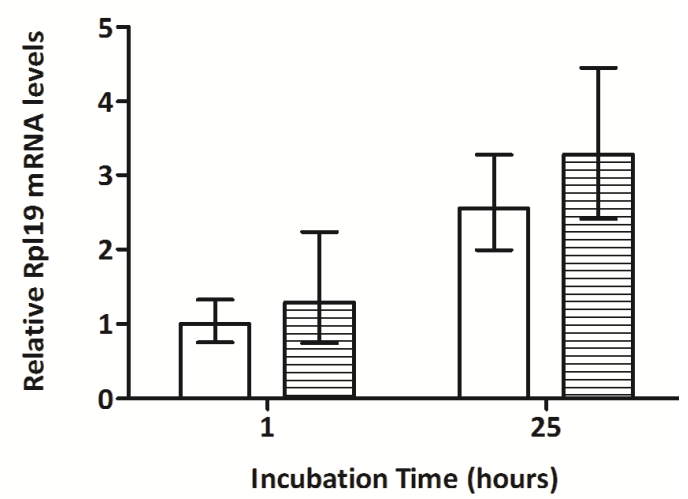

Figure 8-6: Effect of THC on mRNA levels of Atr (A), Cx43 (B), Cycs (C), Gfpt1 (D), Pfkp (E), and Rpl19 (F) in rat CC. The control group is represented by the unfilled bars ( $\square$ ), while the THC-treated group is represented by the hatched bars ( against the mean of the control group at 1 hour. The data were generated from a total of 12 readings from individual oocytes in each group. A two-way ANOVA was performed on untransformed data (B, C, D, E), squared data (A), or square-root transformed data $(F)$. 


\subsection{Discussion}

These results reveal that the function of $\mathrm{CC}$ within rat $\mathrm{COC}$ is influenced by lifestyle or environmental contaminants in the surrounding environment. This study shows that the in vitro exposure of COC to BPA, caffeine, ethanol, MDMA and THC, but not nicotine, resulted in modified expression levels of one or more of CC-derived genes.

The finding that $C_{x} 43$ mRNA levels increased after a long (i.e. 25 hour) but not a short-term (i.e. 1 hour) exposure to BPA indicates that BPA may act indirectly to modify $C x 43$ expression. The increased levels of $C_{x} 43$ mRNA suggest that prolonged exposure to BPA may increase the number of GJs and/or hemichannels present in the CC. Interestingly, exposure to BPA did not affect Atr expression, a gene that mediates the response to DNA damage by causing $G_{2} / M$ arrest. BPA inhibits mitosis in murine $G C$ resulting in $G_{2} / M$ arrest (Xu et al., 2002), however, the Xu study used a dose of BPA that was $>1000$-fold higher than that used in the current study.

Conversely, the exposure of cultured rat $\mathrm{COC}$ to caffeine resulted in an overall reduction in CX43 mRNA in CC. This result was surprising as caffeine is a broad-range PDE inhibitor that has been shown to both promote GJ communication and increase intracellular cAMP levels in rat fibroblasts and epithelial cells in vitro (Flagg-Newton et al., 1981). Indeed, a previous study found that increases in intracellular cAMP levels that induced GJ communication in a rat hepatoma cell line were coincident with increased $C_{x} 43$ mRNA levels (Mehta et al., 1992). Therefore, it was expected that caffeine would increase $C_{x} 43$ levels. However as discussed in Chapter 6, the addition of PDE inhibitors for the entire incubation period in the current study may have maximally-inhibited PDE activity therefore masking any similar effects of caffeine. Thus, the mechanism by which caffeine elicits a reduction of $C_{x} 43$ mRNA may not be related to the PDE inhibitory properties of caffeine.

Exposing rat $\mathrm{COC}$ to ethanol for a short-term period i.e. 1 hour) caused a slight increase in CC-derived $C_{x} 43$ mRNA levels, but following a long term exposure, the mean $C_{x} 43$ mRNA levels were similar to the control. The results presented here suggest that short exposures to ethanol resulted in an increased number of CX43 GJ channels/hemichannels in rat CC. Thereafter, either receptor desensitization or metabolism of ethanol may result in a reduction of $C \times 43$ mRNA to levels similar to that of the control. Ethanol is metabolised by 
alcohol dehydrogenase (ADH) isoenzymes, the expression of which have been confirmed in murine oocytes (Rout and Armant, 2002). However the major isoenzyme that metabolises ethanol, namely ADH1, was not detected. Desensitization of the cellular response to ethanol over long exposure times has previously been reported in a neuroblastoma cell line (Gordon et al., 1986). Initial exposure of neuroblastoma cells to ethanol resulted in increased production of cAMP, however chronic exposure caused desensitization of the heterologous receptors that are coupled to the GTP-binding protein $\mathrm{G}_{\mathrm{S}}$, resulting in reduced cAMP production (Gordon et al., 1986; Mochly-Rosen et al., 1988). The finding in this present study that $C \times 43$ mRNA is increased in rat CC following exposure to ethanol for 1 hour is consistent with increased intracellular cAMP (Mehta et al., 1992). In addition, the finding that the ethanol-mediated increase in $C_{X} 43$ mRNA was attenuated following a 25 hour exposure is consistent with desensitization of rat CC.

Interestingly, nicotine treatment did not significantly affect mRNA levels of any of the candidate genes tested in CC. However, the results presented in this study suggest that in vitro exposure to nicotine may result in a small reduction ( $P=0.052)$ in $C x 43$ mRNA levels in rat CC. This is in contrast with a previous study which found that exposure to nicotine in vitro resulted in increased Cx43 mRNA levels in human umbilical vein endothelial cells (Tsai et al., 2004). However as discussed in Chapter 6, the concentration of nicotine used by the Tsai group was 195 times greater than that used in this study.

The effects of MDMA on gene expression levels in CC were more pronounced than the other aforementioned treatments in that treatment effects or treatment $\mathrm{x}$ time effects were observed in four out of the five candidate genes tested. Expression levels for both $C_{x} 43$ and Gfpt1 mRNA were higher at both time points in the MDMA-treated, compared to control CC. In addition, a time $\mathrm{x}$ treatment interaction was observed for Cycs and Pfkp levels where $P f k p$ levels were higher at 1 hour but lower at 25 hours in the MDMA-treated CC, and Cycs levels were similar at 1 hour but lower at 25 hours in the MDMA-treated CC, compared to controls. These results indicate that MDMA acts directly on CC (i.e. within 1 hour) to regulate gene expression. To the best of my knowledge this is the first study that has examined the effect of MDMA on Cycs, Gfpt1, or Pfkp mRNA levels in any tissue type. The increase in Cx43 mRNA suggests that exposure to MDMA results in an increase in the number of GJs and/or hemichannels present between rat CC which is in contrast to neonatal rat ventricular 
myocytes where MDMA reduced expression levels of Cx43 (Zhuo et al., 2013). Interestingly, these results also strongly suggest that MDMA affects expression of genes involved in glucose metabolism. MDMA has been shown to regulate glucose metabolism in several areas of the brain, although the effects are dependent upon the location within the brain (Obrocki et al., 1999). In the present study, MDMA increased the expression patterns of Gfpt1 and Pfkp at 1 hour, indicating that energy production via the hexosamine and glycolytic pathways was increased. The simultaneous increase of both glucose utilisation rate-limiting enzymes in conjunction with increased $C_{x} 43$ mRNA is possibly due to increased glucose uptake by CC however the expression levels of the glucose transport Glut1 mRNA were below detection limits. Enhanced glucose uptake may lead to an increase in all four glucose utilisation pathways in the COC (i.e. glycolysis, hexosamine, pentose phosphate, and polyol pathways) which is linked with an increase in reactive oxygen species and precocious resumption of nuclear maturation (Sutton-McDowell et al., 2010). Interestingly, Cycs and Pfkp levels were reduced after 25 hours exposure to MDMA. The metabolism of MDMA occurs by two pathways: demethylenation which is catalysed by CYP2D1 and CYP3A2 in rats to produce 3,4-dihydroxymethamphetamine (HHMA); and $\mathrm{N}$-demethylation which is catalysed by CYP1A2 and CYP2D1 to produce 3,4-methylenedioxyamphetamine (MDA) (Green et al., 2003). The four aforementioned CYP enzymes has been detected in the rat ovary (Hiroi et al., 1998; Lee et al., 2012). However, the expression of these enzymes has not been reported in particular ovarian cell types (i.e. the GC, CC, or oocyte). Therefore, a complex interplay between un-metabolised MDMA, HHMA, and MDA may exist to differentially regulate expression levels of $C_{x} 43, C y c s, G f p t 1$, and $P f k p$ in rat CC over time.

The exposure of rat $\mathrm{COC}$ to THC also resulted in an overall treatment effect for both $\mathrm{C}_{x} 43$, and Gfpt1 mRNA levels. The results presented here suggest that more CX43 GJs/hemichannels may be present in the CC following a 25 hour exposure to THC. The finding that THC takes a longer time to modify $C_{X} 43$ mRNA levels, suggests that $C_{X} 43$ is less sensitive than Gfpt1 to THC, or that a THC metabolite is mediating the change in Cx43 levels. The primary enzymes involved in first pass metabolism of THC are CYP2C and CYP3A (Shimada et al., 1994). Almost 100 metabolites of THC have been identified, however the major metabolite is 11-OH-THC (Lemberger et al., 1973; Grotenhermen, 2003). The finding that Gfpt1 levels were upregulated at both time points in the THC-treated CC indicates that 
the activity of the hexosamine biosynthetic pathway may be preferentially increased over the glycolytic pathways. This is a very interesting result as the major glucose utilisation pathway utilised in the $\mathrm{COC}$ is the glycolytic pathway while only a small proportion of glucose is utilised by the hexosamine pathway. The hexosamine pathway is involved in CC expansion, however inappropriate upregulation of this pathway has been linked to compromised developmental competence (Sutton-McDowell et al 2010).

This study reveals that the most sensitive gene of those tested in rat CC was the GJ component $C_{x} 43$. The levels of $C_{x} 43$ were affected by all of the treatments included in this study, although the effect of nicotine did not quite reach significance $(P=0.052)$. It is perhaps unsurprising that this was the case, as mRNA levels of $C_{x} 43$ are rapidly regulated in response to many external stimuli including cAMP (Mehta et al., 1992), prostaglandin $E_{2}$ and parathyroid hormone (Civitelli et al., 1998), and epidermal growth factor (Rivedal et al., 1996). The half-life of most connexins is $<10$ hours (Hervé et al., 2007) suggesting that transcription and translation are important processes by which GJ communication may be regulated. Additionally, transcriptional regulation of the $C_{x} 43$ gene in response to external stimuli may be an important mechanism by which the $\mathrm{CC}$ protect and nurture the oocyte.

In summary, the main findings of this study were that the gene responsible for GJ transfer potential in CC ( $\left.C_{X} 43\right)$ was regulated by most of the compounds tested in this study. Overall, exposure of rat COC to BPA, MDMA, and THC increased CC-derived CX43 mRNA levels but exposure of rat $\mathrm{COC}$ to caffeine, ethanol, and nicotine either lowered $C_{X} 43$ mRNA levels or had little effect. Moreover, exposure of rat COC to MDMA and THC in vitro resulted in an overall increase in Gfpt1 mRNA, whilst a treatment $\mathrm{x}$ time interaction for Cycs and Pfkp was observed following MDMA exposure. 


\section{EFFECTS OF IN VITRO EXPOSURE OF COC TO LIFESTYLE FACTORS ON PROTEIN EXPRESSION}

\subsection{Introduction}

Quantifying the expression level of a gene that encodes a particular protein only gives an indication of how much protein a cell is instructed to synthesise. Subsequent posttranscriptional and post-translational modifications may further enhance or restrict the level of biologically-active protein that is available. Thus, while quantification of gene expression levels are very informative, a true account of the amount of protein being synthesised at any one time is only obtained by measuring protein expression levels. The synthesis of both CX43 and GDF9 proteins are regulated by post-translational events.

GJ communication is regulated in a dynamic manner throughout folliculogenesis by gonadotrophins (Granot and Dekel, 2002), CAMP and PKA (Yogo et al., 2002), and MAPK (Norris et al., 2008). Rats treated with pregnant mare's serum gonadotrophin had increased levels of CX43 protein whereas an additional hCG injection resulted in a marked reduction in CX43 levels (Granot and Dekel, 1997). The CX43 gene contains two exons separated by a 30 kilobase intron; the first exon contains a $5^{\prime}$-untranslated region (UTR), while the second exon contains the protein coding region and a $3^{\prime}$-UTR. The first exon is differentially spliced to form at least nine different $C_{x 43}$ species in mice, and six different species in rat (Pfeifer et al., 2004). These $C \times 43$ species had varying translation efficiencies both between different species and in different cell lines. Therefore, as previously stated, levels of $C_{x} 43$ gene expression may not correlate with protein levels.

An important mechanism by which GJ communication is regulated is through phosphorylation of connexin proteins. For example, several forms of CX43 are often detected using Western blotting, including a non-phosphorylated form (P0), and two differentially phosphorylated forms (P1 and P2) that migrate at a slower rate (Solan and Lampe, 2009). Phosphorylation on $\mathrm{Ser}^{365}$ is responsible for the altered migration of the P1 form (Solan et al., 2007), while phosphorylation at $\operatorname{Ser}^{325}, \operatorname{Ser}^{328}$, and $\operatorname{Ser}^{330}$ is responsible for the altered migration of the P2 form (Lampe et al., 2006). These phosphorylation sites are 
thought to be involved in promoting GJ formation and activity (Yogo et al., 2002; Solan and Lampe, 2009).

GDF9 is the primary oocyte-secreted factor involved in regulation of CC/GC function in rodents (Lin et al., 2012). Post-translational modifications are required for GDF9 signalling. As with other members of the TGF $\beta$ family, GDF9 is initially synthesised as a preproprotein. The signal peptide is removed upon secretion from the oocyte and the promature protein is released into the follicular fluid in humans, mice and sheep (Gilchrist et al., 2004b; McNatty et al., 2006; Gode et al., 2011). The proregions of TGF $\beta$ proteins are cleaved by proprotein convertase subtilisin/kexin enzymes (Harrison et al., 2011) and evidence exists that this cleavage occurs both intracellularly and extracellularly (Blanchet et al., 2008; Susan-Resiga et al., 2011). Generally, the mature protein has been considered to be the bioactive form. However, a recent study suggests that the proregion also plays an important role in female reproduction. When female mice were immunized with a GDF9 proregion peptide, these mice had more corpora lutea but smaller litter sizes (McIntosh et al., 2012). The exact role of the proregion is unknown, however for other TGF $\beta$ proteins it mediates dimer formation and targets the protein to the receptor (Sengle et al., 2008; Walton et al., 2009).

Therefore, while transcriptional regulation of gene expression is an important indicator of an effect that a treatment has on a cell, this does not always correspond to what occurs at a protein level (Gygi et al., 1999). In addition, post-translational modifications play an important role in protein bioactivity and function.

The aim of this chapter was to determine the effect of in vitro exposure of rat COC to BPA, caffeine, ethanol, nicotine, MDMA, or THC on the expression levels of CC-derived CX43 and oocyte-derived GDF9 proteins.

\subsection{Methods}

\subsubsection{COC collection}

Ovaries from prepubescent Sprague-Dawley rats were collected in PBS and the COC were extracted as previously described (Section 2.1.2). The $\mathrm{COC}$ were incubated either with treatment or without treatment (control group) for 1 or 25 hours at $37^{\circ} \mathrm{C}$ with $5 \% \mathrm{CO}_{2}$ and $96 \%$ humidity, to mimic the incubation conditions used in the GJ assay (see Chapter 6 and 
Table 6-1). Subsequently, 100 COC were washed in PBS before being transferred into a microcentrifuge tube. The tubes were centrifuged at $800 \times g$ for 10 minutes, the supernatant removed, and the COC resuspended in PBS supplemented with protease inhibitors. Each COC collection experiment was performed twice, and the samples were pooled so that the final number of $\mathrm{COC}$ in each sample was 200 . The samples were stored at $-20^{\circ} \mathrm{C}$ until further processing.

\subsubsection{BCA Assay}

Total protein concentration was measured using the BCA assay (Section 2.4.1). Briefly, a bovine serum albumin standard was serially diluted in PBS and $25 \mu \mathrm{L}$ was added in triplicate into a 96-well plate. One third of each COC sample ( 67 COC in $\sim 10 \mu \mathrm{L}$ ) was serially diluted in PBS and aliquoted in duplicate into a 96-well plate. BCA working reagent (200 $\mu \mathrm{L})$ was added to each well, and the plate was then incubated at $37^{\circ} \mathrm{C}$ (original assays) or $60^{\circ} \mathrm{C}$ (subsequent assays) for 2 hours. The plates were cooled to room temperature and the absorbance at 490 $\mathrm{nm}$ was measured using a VICTOR3 multilabel plate reader (PerkinElmer; Surrey Hills, Australia). The concentration of protein in each COC sample was calculated using the equation obtained from the bovine serum albumin standard curve.

\subsubsection{Western blotting}

Two-thirds of each COC sample ( 133 COC in $\sim 20 \mu \mathrm{L}$ ) was added to sample buffer, incubated at $95^{\circ} \mathrm{C}$ in a water bath for 10 minutes, before being loaded onto a polyacrylamide gel. The proteins were separated according to size by SDS-PAGE, transferred onto a nitrocellulose membrane, and CX43 or GDF9 protein expression was detected using Western blotting. In brief, CX43 was identified using the monoclonal antibody MAB3067. To determine MAB3067 specificity, the antibody was pre-absorbed with 5x the corresponding MAB3067 control peptide. GDF9 was detected using a monoclonal antibody (MAB47B) which was generated against the C-terminal region that corresponds to the mature region of ovine GDF9. To determine MAB47B specificity, the antibody was pre-absorbed with 5x E. coli produced recombinant ovine GDF9. The membranes were incubated with electrochemiluminescence reagents for 1 minute before being exposed to an X-ray film for between 1 and 10 minutes. 


\subsubsection{Data analysis}

The density of the bands present on the Western blots was measured using Image J software. For the CX43 blots, the density of the $43 \mathrm{kDa}$ band was measured. For the GDF9 blots, the densities of the $49 \mathrm{kDa}$ promature and, when present, the $20 \mathrm{kDa}$ mature bands were measured. When multiple bands were present (e.g. when doublet or triplet bands were present for $\mathrm{CX} 43$ ) the density of all bands was measured. The total protein amount in each sample was used to correct for CX43, but not GDF9, protein levels. The reasoning behind this was that samples contained entire COCs which consisted mostly of large numbers of CC and a few (i.e. 200) oocytes. Thus, differences in total protein levels between samples would predominantly be due to differences in the number of CC, and not oocytes. Therefore, CX43 protein levels are referred to as corrected levels and GDF9 protein levels are referred to as uncorrected levels.

Each experiment comprised of 200 COC for each experimental group at each time point, therefore there were only two replicated experimental samples at each time point. Thus statistical analysis was inappropriate and therefore interpretation of the data is simply based on the evidence of two replicate but independent studies.

\subsection{Results}

\subsubsection{Effect of BPA and caffeine on protein levels in rat COC}

The effects of in vitro exposure of rat COC to $20 \mathrm{ng} / \mathrm{mL}$ BPA or $100 \mu \mathrm{g} / \mathrm{mL}$ caffeine on CX43 protein levels are depicted in Figure 9-1. A $43 \mathrm{kDa}$ band was observed in the positive control in both experimental replicates, while no bands were observed in the negative control in either replicate. There was some non-specific background signal in the first replicate (Figure 9-1A), however this did not obscure the bands of interest. A $43 \mathrm{kDa}$ band was observed in each of the lanes containing COC lysate. When the CX43 antibody was pre-absorbed with the control peptide, no bands were detected (Figure 9-1C).

In the first replicate experiment (Figure 9-1A), a 1.7-fold reduction in CX43 levels with time in culture was noted in the control COC. Following exposure to BPA and caffeine for 1 hour, CX43 levels were 2.0-fold and 1.8-fold greater than the levels in the control at that time. Similar to the control, CX43 levels decreased over time in the BPA-treated and caffeine- 
treated COC whereby a 10-fold and 1.8-fold reduction in CX43 levels over time were observed for these treatments so that levels were 3-fold lower and 1.7-fold greater than the levels in the control at 25 hours.

The treatment responses in the second replicate relative to the control differed somewhat from the first replicate but the CX43 levels over time followed the same pattern in all treatment groups (Figure 9-1B). CX43 levels in the control COC declined 10-fold with time in culture. CX43 levels were 1.4-fold lower or 1.4-fold greater after incubation with BPA and caffeine for 1 hour, compared to the control. Similar to the control, 1.8-fold and 7-fold reductions in CX43 levels were observed with time in culture so that levels were 4-fold and 2-fold greater than that in the control following 25 hours incubation with BPA and caffeine, respectively.

Regardless of treatment, there was a consistent decrease in the mean CX43 levels in cultured $\mathrm{COC}$ with time (Figure 9-2). CX43 levels following exposure to BPA were not different from that in the control, although there was variability between the BPA treated replicates after 1 hour of culture. Exposure of COC to caffeine after 1 hour resulted in a reproducible increase in CX43 levels compared to controls, however the overall decline in expression over 25 hours was similar to that noted in the control and BPA treated groups. 
A

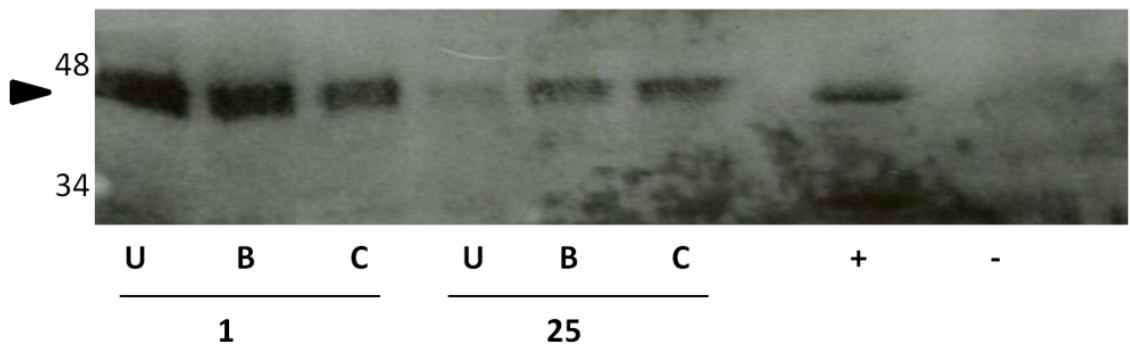

\begin{tabular}{lcccccccc}
\hline $\begin{array}{l}\text { Uncorrected } \\
\text { band density }\end{array}$ & 1.0 & 1.0 & 0.6 & 0.1 & 0.3 & 0.4 & - & - \\
\hline $\begin{array}{l}\text { Total protein } \\
\text { content }\end{array}$ & 0.9 & 0.5 & 0.3 & 0.2 & 1.6 & 0.4 & - & - \\
\hline $\begin{array}{l}\text { Band density/ } \\
\text { protein content }\end{array}$ & 1.0 & 2.0 & 1.8 & 0.6 & 0.2 & 1.0 & - & - \\
\hline
\end{tabular}

B

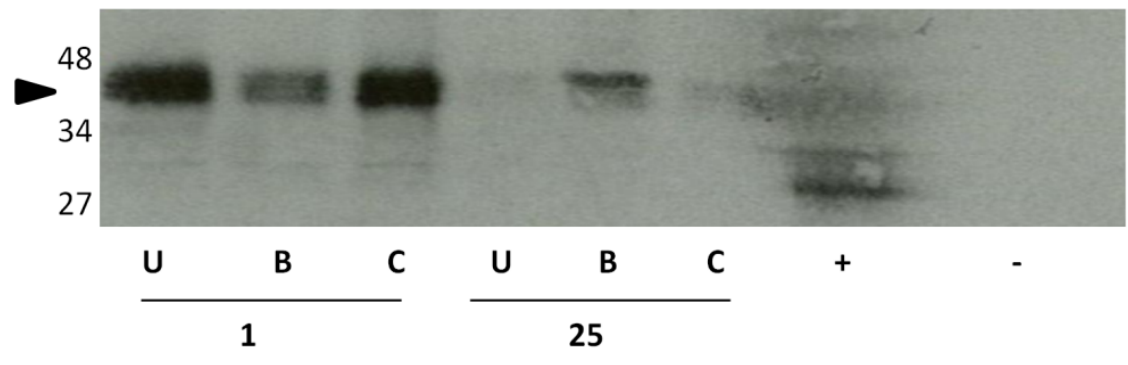

\begin{tabular}{lcccccccc}
\hline $\begin{array}{l}\text { Uncorrected } \\
\text { band density }\end{array}$ & 1.0 & 0.5 & 0.9 & 0.03 & 0.4 & 0.1 & - & - \\
\hline $\begin{array}{l}\text { Total protein } \\
\text { content }\end{array}$ & 2.3 & 1.7 & 1.5 & 0.8 & 1.9 & 1.3 & - & - \\
\hline $\begin{array}{l}\text { Band density/ } \\
\text { protein content }\end{array}$ & 1.0 & 0.7 & 1.4 & 0.1 & 0.4 & 0.2 & - \\
\hline
\end{tabular}

C

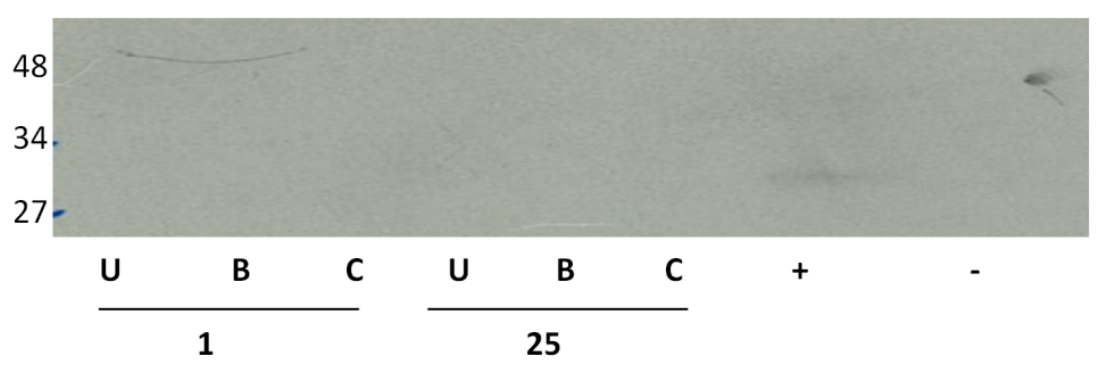

Figure 9-1: The effects of exposing rat COC to BPA and caffeine in vitro on CX43 protein levels over time. Western blots were performed in duplicate (A and B) to determine the level of CX43 protein in COC lysate. COC were either incubated for 1 or 25 hours with the solvent control (U), BPA (B), or caffeine (C). Rat ovary lysate was included as a positive control $(+)$ and $E$. coli produced recombinant ovine BMP15 was included as a negative control (-). A control experiment (C) was also performed where the blot shown in (B) was stripped and re-probed with the CX43-specific antibody that had been pre-absorbed with its control peptide. Molecular sizes $(\mathrm{kDa})$ are indicated to the left of each blot. The density of the band of interest (indicated by an arrowhead) was measured and corrected for the control at 1 hour. Total protein $(\mu \mathrm{g})$ in each sample was determined using the BCA assay. The relative band densities were corrected for total protein amount, as calculated by the BCA assay. 


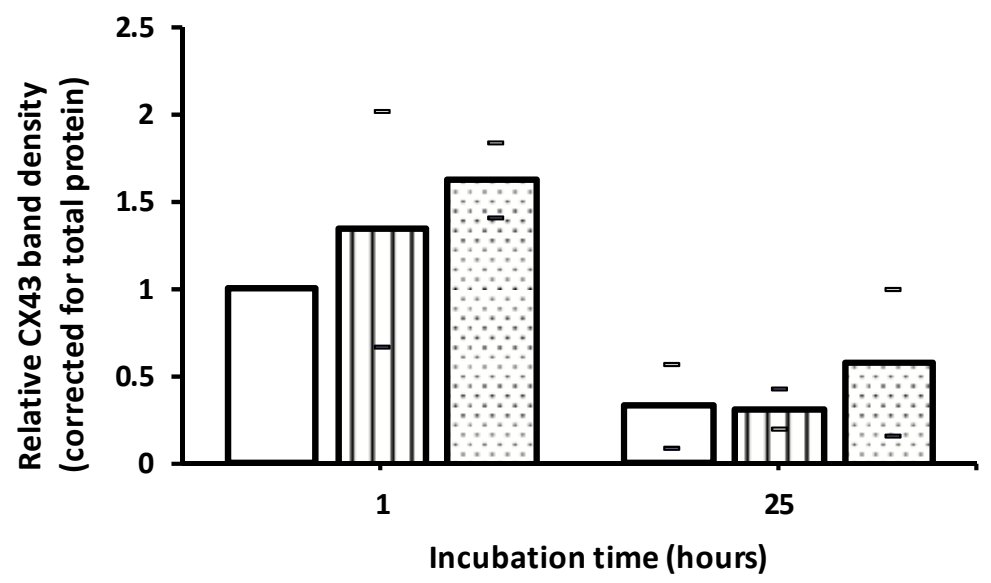

Figure 9-2: Effect of BPA and caffeine on relative CX43 levels in rat COC. The control group is represented by the unfilled bars $(\square)$, the BPA-treated group is represented by the striped bars ( $\square$ ), while the caffeinetreated group is represented by the dotted bars $(\cdots)$. The bars represent the means, while the dashes represent each individual band density measurement.

The effects of exposing rat COC to $20 \mathrm{ng} / \mathrm{mL}$ BPA or $100 \mu \mathrm{g} / \mathrm{mL}$ caffeine on GDF9 protein levels are depicted in Figure 9-3. Multiple bands between 20 and 114 kDa were detected in the positive control in both replicates. The bands in the positive control of the first replicate (Figure 9-3A) were overexposed, so most individual bands were unable to be distinguished. Bands of 49 and $20 \mathrm{kDa}$ in size, corresponding to the promature and mature forms of GDF9 respectively, were detected in the positive control lanes. Additional prominent bands of 93, 70 , and $40 \mathrm{kDa}$ were observed. No bands were observed in the negative control. When the GDF9 antibody was pre-absorbed with recombinant GDF9, no bands were observed (Figure 9-3C). Multiple bands corresponding to the aforementioned molecular weights observed in the positive controls were observed in each of the lanes containing COC lysate.

In the first replicate experiment (Figure 9-3A), promature GDF9 in control COC declined 5fold over time. Promature GDF9 levels were 2-fold and 3.3-fold lower than the control following incubation with BPA and caffeine for 1 hour. In contrast to the pattern of promature GDF9 levels over time in the control, the amount of promature GDF9 in the BPAtreated and caffeine-treated COC increased 1.6-fold or 2.7-fold with time so that levels were 4-fold greater in both the BPA- and caffeine-treated groups than that in the control at 25 hours. Mature GDF9 protein was only detected in the control COC at 1 hour and in the treated COC at 25 hours. 
In the second replicate experiment (Figure 9-3B), promature GDF9 levels in control COC decreased slightly (1.3-fold) with time. Promature GDF9 levels were 3.3-fold and 1.3-fold lower than the control following 1 hour exposure to BPA and caffeine respectively. In contrast to the pattern of promature GDF9 levels with time in the control, levels of promature GDF9 levels increased 6-fold and 2.4-fold with time in the BPA-treated and caffeine-treated COC, so that levels were 2.3-fold and 2.4-fold greater than the control at 25 hours. Similar to the first replicate, mature GDF9 was only detected in the control COC at 1 hours and the treated $\mathrm{COC}$ at 25 hours.

Overall in the control COC, the mean promature GDF9 levels declined over time (Figure 9-4), notwithstanding the variability between the replicates after 25 hours in culture. Compared to the control, the mean levels of promature GDF9 for the BPA-treated and caffeine-treated group were lower after 1 hour in culture but both had recovered to levels above that in the controls after 25 hours in culture. 
A

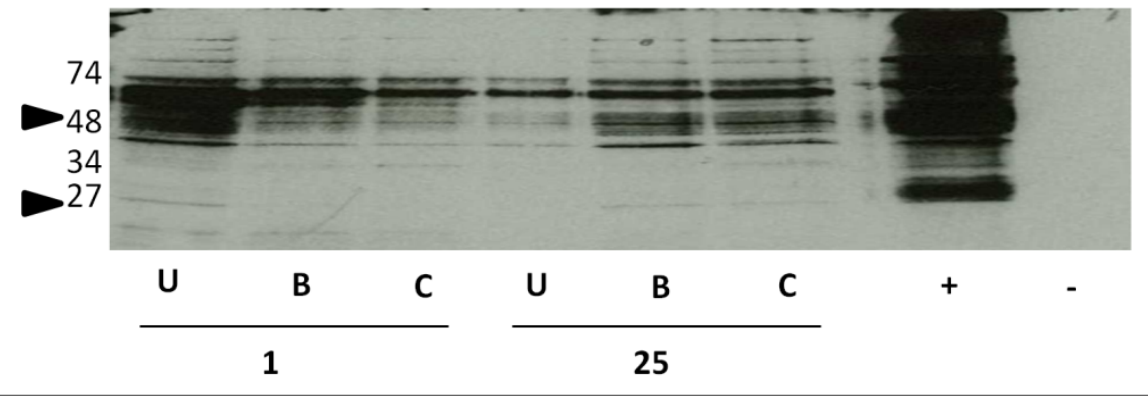

\begin{tabular}{lcccccccc}
\hline $\begin{array}{l}\text { Uncorrected promature } \\
\text { band density }\end{array}$ & 1.0 & 0.5 & 0.3 & 0.2 & 0.8 & 0.8 & - & - \\
\hline $\begin{array}{l}\text { Uncorrected mature } \\
\text { band density }\end{array}$ & 1.0 & 0.0 & 0.0 & 0.0 & 0.7 & 0.7 & - & - \\
\hline Total protein content & 5.0 & 4.4 & 3.7 & 4.3 & 4.6 & 5.2 & - & - \\
\hline
\end{tabular}

B
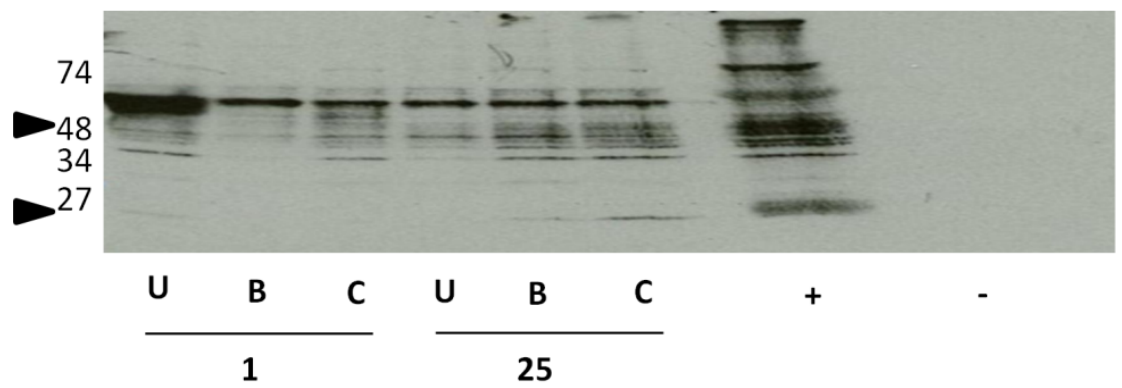

\begin{tabular}{|c|c|c|c|c|c|c|c|c|}
\hline $\begin{array}{l}\text { Uncorrected promature } \\
\text { band density }\end{array}$ & 1.0 & 0.3 & 0.8 & 0.8 & 1.8 & 1.9 & - & - \\
\hline $\begin{array}{l}\text { Uncorrected mature } \\
\text { band density }\end{array}$ & 1.0 & 0.0 & 0.0 & 0.0 & 1.2 & 5.6 & - & - \\
\hline Total protein content & 4.4 & 2.8 & 4.5 & 3.1 & 4.9 & 5.3 & - & - \\
\hline
\end{tabular}

C

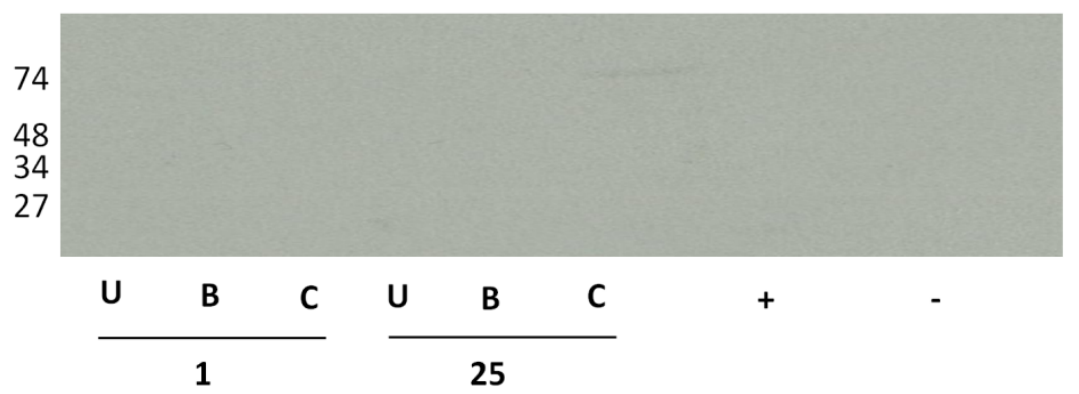

Figure 9-3: The effects of exposing rat COC to BPA and caffeine in vitro on GDF9 protein levels over time. Western blots were performed in duplicate ( $A$ and $B$ ) to determine the level of GDF9 protein in COC lysate. COC were either incubated for 1 or 25 hours with the solvent control (U), BPA (B), or caffeine (C). Rat ovary lysate was included as a positive control $(+)$ and $E$. coli produced recombinant ovine BMP15 was included as a negative control (-). A control experiment (C) was also performed where the blot shown in (B) was stripped and re-probed with the GDF9-specific antibody that had been pre-absorbed with $E$. coli produced recombinant ovine GDF9. Molecular sizes $(\mathrm{kDa})$ are indicated to the left of each blot. The densities of the bands of interest (indicated by arrowheads) were measured and corrected for the control at 1 hour. Total protein ( $\mu \mathrm{g}$ ) in each sample was determined using the BCA assay. 


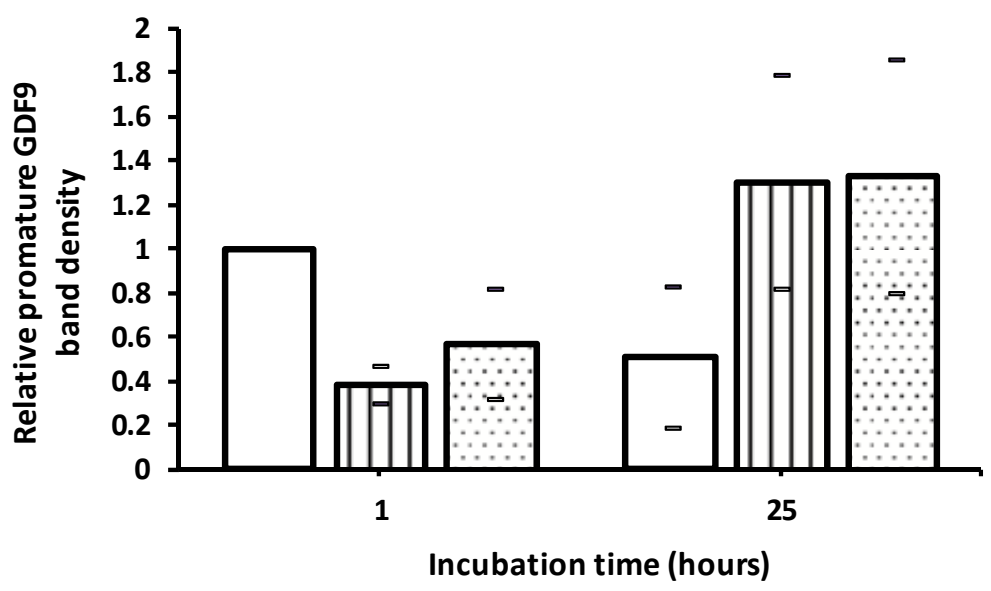

Figure 9-4: Effect of BPA and caffeine on relative GDF9 levels in rat COC. The control group is represented by the unfilled bars $(\square)$, the BPA-treated group is represented by the striped bars ( $\square$ ), while the caffeinetreated group is represented by the dotted bars $(\cdots)$. The bars represent the means, while the dashes represent each individual band density measurement.

\subsubsection{Effect of ethanol on protein levels in rat COC}

The effect of exposing COC to $7.5 \mathrm{mg} / \mathrm{mL}$ ethanol in vitro on CX43 protein levels is depicted in Figure 9-5. A $43 \mathrm{kDa}$ band was observed in the positive control of both experimental replicates, while no bands were observed in the negative control of either. A $43 \mathrm{kDa}$ band was observed in each of the lanes containing COC lysate. Multiple smaller bands were also present in all lanes however the identities of these bands are unknown. When the CX43 antibody was pre-absorbed with the control peptide no bands were observed (Figure 9-5C).

In the first replicate experiment (Figure 9-5A), CX43 levels in control COC did not change over time. CX43 levels were similar between the ethanol-treated and control COC at 1 hour. In the ethanol-treated $\mathrm{COC}$, the CX43 levels decreased slightly (1.4-fold) over time and were 1.3-fold lower after 25 hours of culture compared to the controls.

A similar response was observed in the second replicate experiment (Figure 9-5B). Levels of CX43 in the control COC did not change over time. After ethanol exposure CX43 levels were 1.3-fold greater after 1 hour, compared to the control and by 25 hours had decreased 1.6fold at which time the levels were 1.3-fold lower than that measured in the control.

Overall in the control COC, CX43 levels did not change with time in culture (Figure 9-6). However in the ethanol-treated COC, there was a consistent reduction in CX43 levels after 25 hours in culture, however this was negligible. 
A

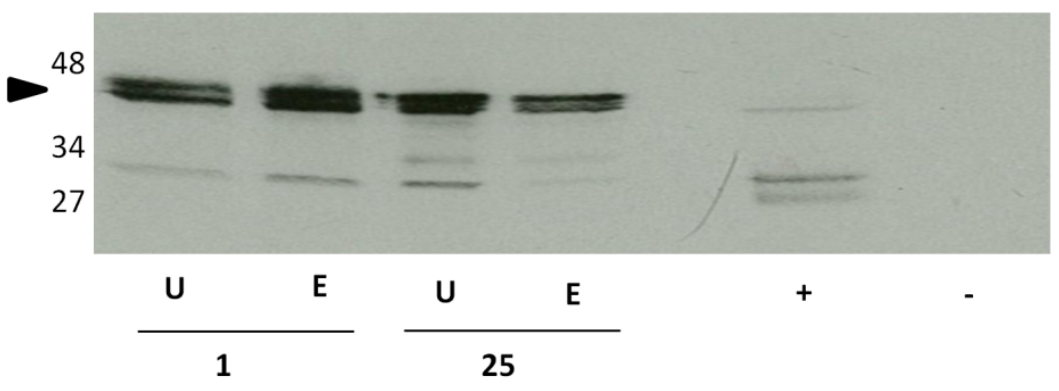

\begin{tabular}{lcccccc}
\hline $\begin{array}{l}\text { Uncorrected } \\
\text { band density }\end{array}$ & 1.0 & 1.0 & 1.2 & 0.8 & - & - \\
\hline $\begin{array}{l}\text { Total protein } \\
\text { content }\end{array}$ & 19.5 & 18.1 & 22.9 & 21.3 & - & - \\
\hline $\begin{array}{l}\text { Band density/ } \\
\text { protein content }\end{array}$ & 1.0 & 1.1 & 1.0 & 0.8 & - \\
\hline
\end{tabular}

B

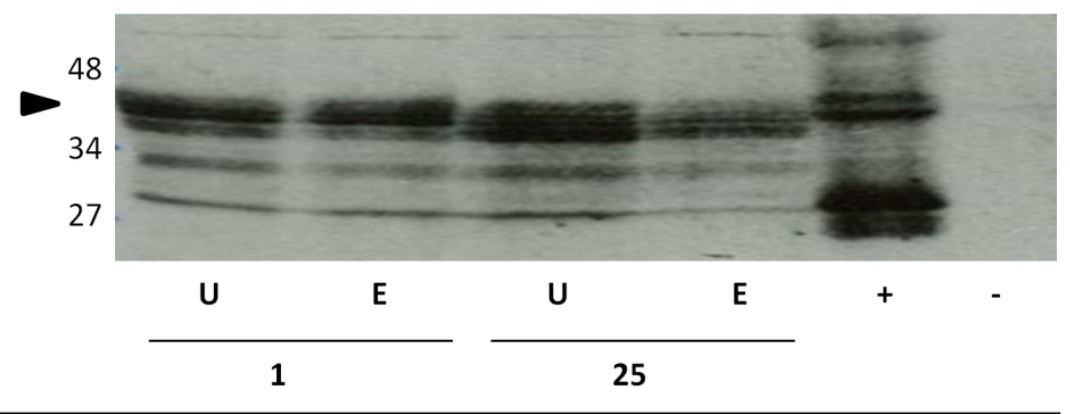

\begin{tabular}{lcccccc}
\hline $\begin{array}{l}\text { Uncorrected } \\
\text { band density }\end{array}$ & 1.0 & 1.3 & 1.1 & 0.8 & - & - \\
\hline Total protein content & 6.0 & 5.9 & 6.7 & 5.9 & - & - \\
\hline $\begin{array}{l}\text { Band density/ } \\
\text { protein content }\end{array}$ & 1.0 & 1.3 & 1.0 & 0.8 & - & - \\
\hline
\end{tabular}

C

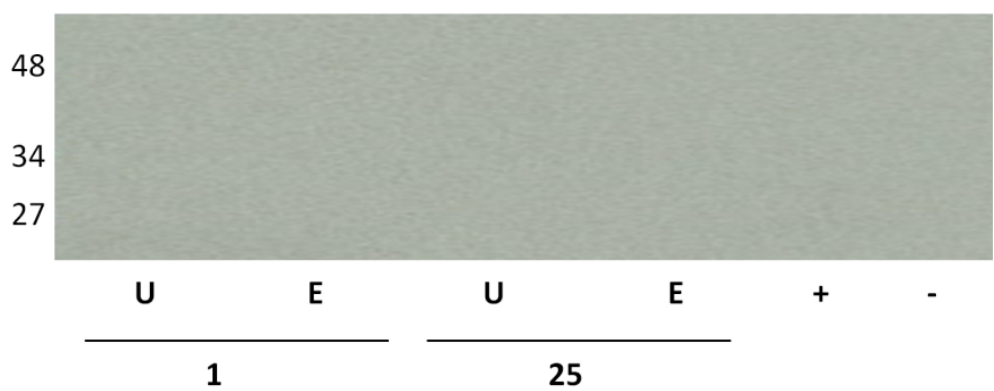

Figure 9-5: The effects of exposing rat COC to ethanol in vitro on CX43 protein levels over time. Western blots were performed in duplicate (A and B) to determine the level of CX43 protein in COC lysate. COC were either incubated for 1 or 25 hours with the solvent control (U) or ethanol (E). Rat ovary lysate was included as a positive control (+) and E. coli produced recombinant ovine BMP15 was included as a negative control (-). A control experiment $(C)$ was also performed where the blot shown in (B) was stripped and re-probed with the CX43-specific antibody that had been pre-absorbed with its control peptide. Molecular sizes (kDa) are indicated to the left of each blot. The density of the band of interest (indicated by an arrowhead) was measured and corrected for the control at 1 hour. Total protein $(\mu \mathrm{g})$ in each sample was determined using the BCA assay. The relative band densities were corrected for total protein amount, as calculated by the BCA assay. 


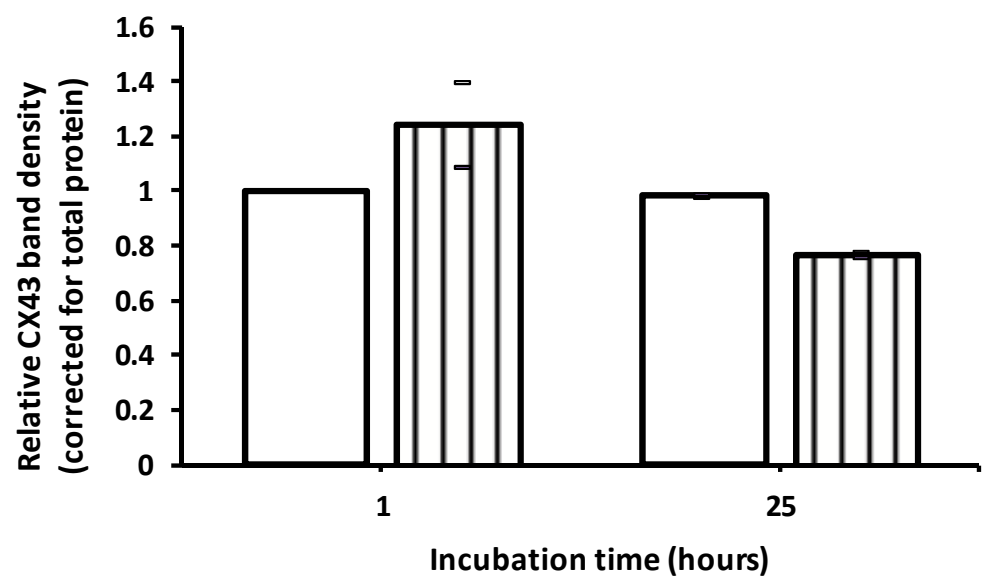

Figure 9-6: Effect of ethanol on relative CX43 levels in rat COC. The control group is represented by the unfilled bars ( $\square$ ), while the ethanol-treated group is represented by the striped bars ( $\square$ ). The bars represent the means, while the dashes represent each individual band density measurement.

The effect of exposing rat COC to $7.5 \mathrm{mg} / \mathrm{mL}$ ethanol on GDF9 protein levels are depicted in Figure 9-7. Multiple bands between 20 and 114 kDa were detected in the positive control of both replicates. The bands in the positive control of the first replicate (Figure 9-7A) were overexposed, so most individual bands were unable to be distinguished. Bands of 49 and 20 $\mathrm{kDa}$ in size, corresponding to the promature and mature forms of GDF9 respectively were detected in the positive control lanes. Additional prominent bands of 93, 70, and $40 \mathrm{kDa}$ were observed. No bands were observed in the negative control. When the GDF9 antibody was pre-absorbed with recombinant GDF9, no bands were detected (Figure 9-7C). Multiple bands were observed in each of the lanes containing COC lysate, corresponding to the aforementioned molecular weights in the positive control.

In the first replicate (Figure 9-7A), levels of promature GDF9 increased 2.4-fold over time in the controls. Promature GDF9 levels were 1.4-fold lower in ethanol-treated COC at 1 hour, compared to the control. Similar to the control, levels of promature GDF9 in the ethanoltreated COC increased 3.4-fold over time such that levels were similar between the ethanoltreated and control COC at 25 hours.

In the second replicate, levels of promature GDF9 increased 1.6-fold over time in the controls (Figure 9-7B). Promature GDF9 levels were 1.3-fold lower following incubation with ethanol for 1 hour, compared to the control. In the ethanol-treated COC, levels of promature 
GDF9 only increased slightly (1.3-fold) with time in culture so that levels were 1.6-fold lower at 25 hours, compared to the control.

Overall, levels of promature GDF9 consistently increased after 25 hours in culture in both the control and ethanol-treated samples (Figure 9-8). However, no difference in the levels of promature GDF9 following incubation treatment were noted. 
A

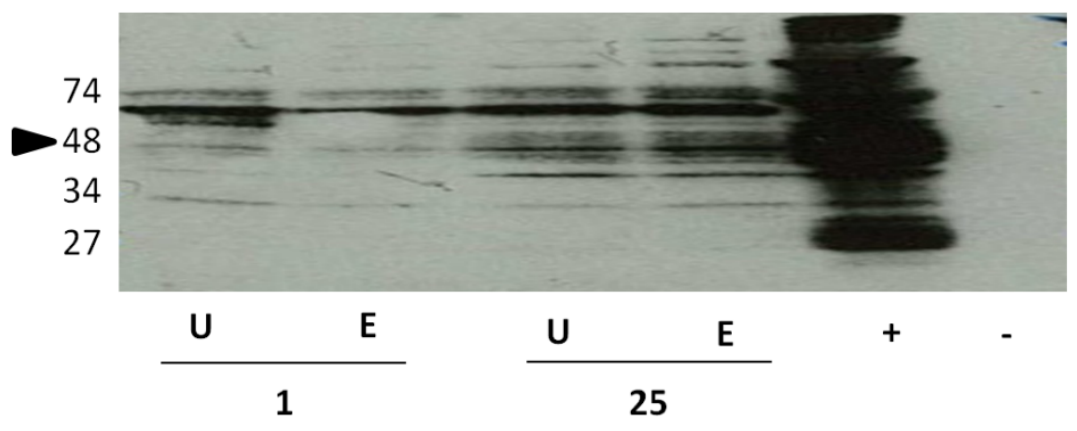

\begin{tabular}{lllllll}
\hline $\begin{array}{l}\text { Uncorrected promature } \\
\text { band density }\end{array}$ & 1.0 & 0.7 & 2.4 & 2.4 & - & - \\
\hline Total protein content & 4.4 & 3.7 & 4.4 & 4.7 & - & - \\
\hline
\end{tabular}

B

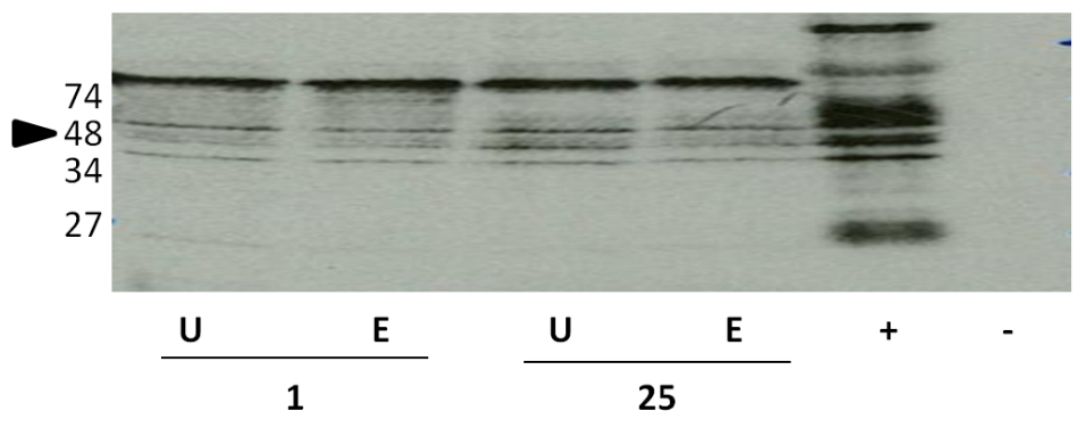

\begin{tabular}{lcccccc}
\hline $\begin{array}{l}\text { Uncorrected promature } \\
\text { band density }\end{array}$ & 1.0 & 0.8 & 1.6 & 1.0 & - & - \\
\hline Total protein content & 19.5 & 18.2 & 22.9 & 21.3 & - & - \\
\hline
\end{tabular}

C

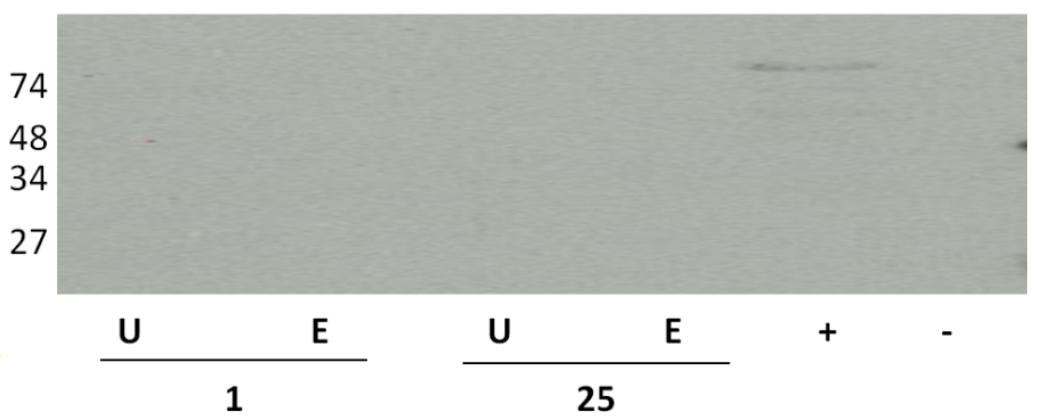

Figure 9-7: The effects of exposing rat COC to ethanol in vitro on GDF9 protein levels over time. Western blots were performed in duplicate (A and B) to determine the level of GDF9 protein in COC lysate. COC were either incubated for 1 or 25 hours with the solvent control (U) or ethanol (E). Rat ovary lysate was included as a positive control $(+)$ and E. coli produced recombinant ovine BMP15 was included as a negative control (-). A control experiment (C) was also performed where the blot shown in (B) was stripped and re-probed with the GDF9-specific antibody that had been pre-absorbed with E. coli produced recombinant ovine GDF9. Molecular sizes $(\mathrm{kDa})$ are indicated to the left of each blot. The density of the band of interest (indicated by an arrowhead) was measured and corrected for the control at 1 hour. Total protein $(\mu \mathrm{g})$ in each sample was determined using the BCA assay. 


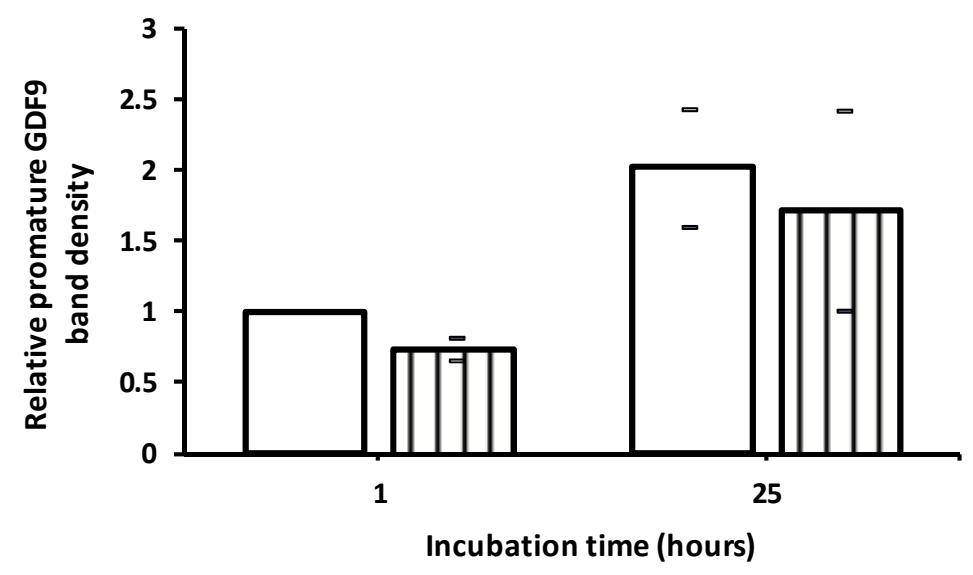

Figure 9-8: Effect of ethanol on relative GDF9 levels in rat COC. The control group is represented by the unfilled bars ( $\square$ ), while the ethanol-treated group is represented by the striped bars ( $\square$ ). The bars represent the means, while the dashes represent each individual band density measurement.

\subsubsection{Effect of nicotine on protein levels in rat COC}

The effect of exposing rat COC to $500 \mathrm{ng} / \mathrm{mL}$ nicotine on CX43 protein levels is depicted in Figure 9-9. A $43 \mathrm{kDa}$ band was observed in the positive control of both experimental replicates, while no bands were observed in the negative control of either replicate. A 43kDa band was observed in each of the lanes containing COC lysate. Multiple smaller bands were also present in all lanes however the identities of these bands are unknown. When the CX43 antibody was pre-absorbed with the control peptide, the bands that had previously been visualised disappeared (Figure 9-9C).

In the first replicate experiment (Figure 9-9A), CX43 levels decreased 5-fold over time in the control group. Levels of CX43 were 5-fold lower after incubation with nicotine for 1 hour when compared to the control group. After 25 hours of culture, the CX43 levels increased 2.5-fold over time in the nicotine-treated group such that levels were 2.5 -fold greater in the nicotine-treated group compared to the control at 25 hours.

A similar response was observed in the second replicate experiment (Figure 9-9B), CX43 levels decreased 2.5-fold over time in the control group. Similar to the first replicate experiment, CX43 levels were 5-fold lower in COC incubated with nicotine for 1 hour compared to the control. CX43 levels increased 7.5-fold over time in the nicotine-treated COC increased, such that levels were 3.8-fold greater in the nicotine-treated group compared to the control at 25 hours. 
Overall, there was a consistent decrease in CX43 levels with time in culture in the control group (Figure 9-10). Conversely, CX43 levels were markedly lower at 1 hour incubation in nicotine-treated $\mathrm{COC}$ compared to the controls but were higher than the equivalent controls after 25 hours of incubation. 
A

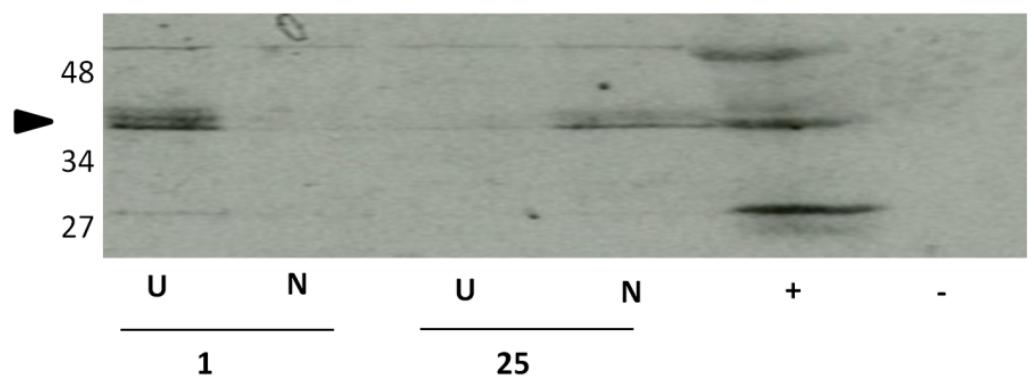

\begin{tabular}{lcccccc}
\hline $\begin{array}{l}\text { Uncorrected } \\
\text { band density }\end{array}$ & 1.0 & 0.2 & 0.2 & 0.5 & - & - \\
\hline $\begin{array}{l}\text { Total protein } \\
\text { content }\end{array}$ & 4.8 & 4.2 & 4.7 & 4.7 & - & - \\
\hline $\begin{array}{l}\text { Band density/ } \\
\text { protein content }\end{array}$ & 1.0 & 0.2 & 0.2 & 0.5 & - & - \\
\hline
\end{tabular}

B

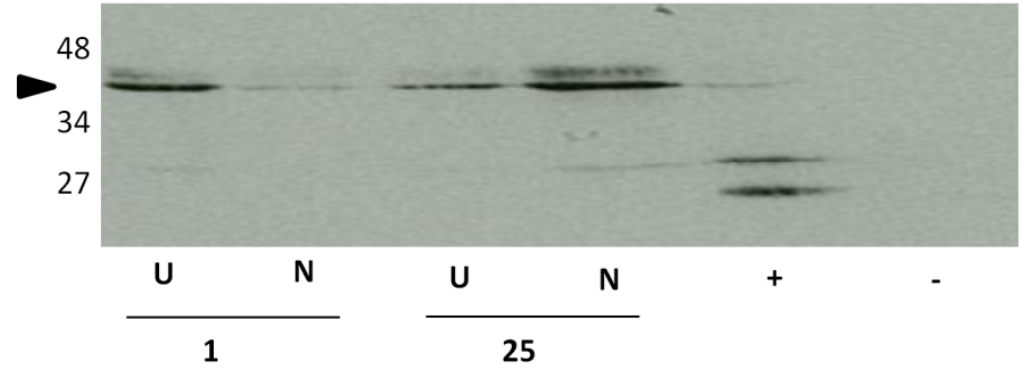

\begin{tabular}{lccccc}
\hline $\begin{array}{l}\text { Uncorrected } \\
\text { band density }\end{array}$ & 1.0 & 0.1 & 0.5 & 1.6 & - \\
\hline $\begin{array}{l}\text { Total protein } \\
\text { content }\end{array}$ & 4.7 & 3.5 & 5.2 & 5.0 & - \\
\hline $\begin{array}{l}\text { Band density/ } \\
\text { protein content }\end{array}$ & 1.0 & 0.2 & 0.4 & 1.5 & - \\
\hline
\end{tabular}

C

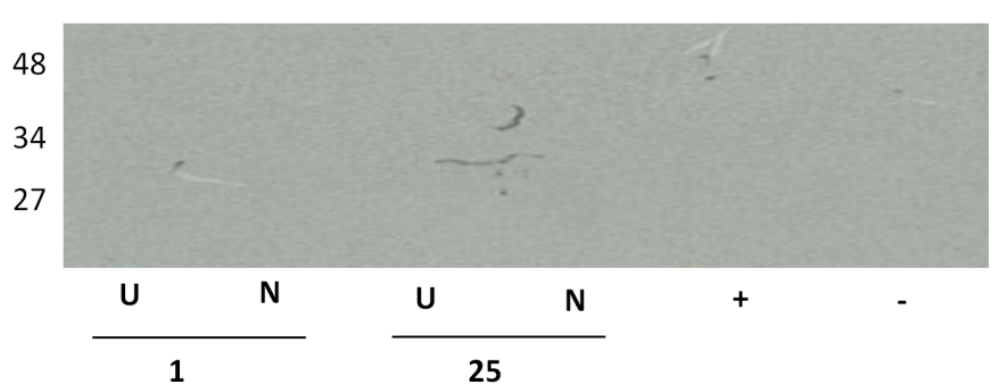

Figure 9-9: The effects of exposing rat COC to nicotine in vitro on CX43 protein levels in rat COC over time. Western blots were performed in duplicate ( $A$ and $B$ ) to determine the level of CX43 protein in COC lysate. COC were either incubated for 1 or 25 hours with the solvent control $(U)$ or nicotine $(N)$. Rat ovary lysate was included as a positive control $(+)$ and $E$. coli produced recombinant ovine BMP15 was included as a negative control (-). A control experiment (C) was also run where the blot shown in (B) was stripped and re-probed with the CX43-specific antibody that had been pre-absorbed with its control peptide. Molecular sizes (kDa) are indicated to the left of each blot. The density of the band of interest (indicated by an arrowhead) was measured and corrected for the control at 1 hour. Total protein $(\mu \mathrm{g})$ in each sample was determined using the $\mathrm{BCA}$ assay. The relative band densities were corrected for total protein amount, as calculated by the BCA assay. 


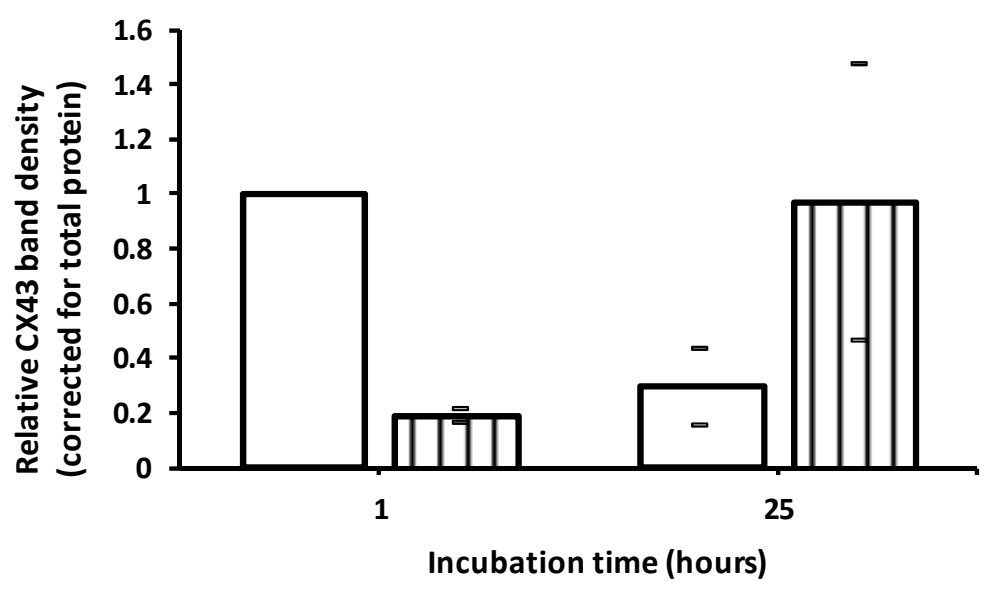

Figure 9-10: Effect of nicotine on relative CX43 levels in rat COC. The control group is represented by the unfilled bars ( $\square$ ), while the nicotine-treated group is represented by the striped bars ( $\square$ ). The bars represent the means, while the dashes represent each individual band density measurement.

The effects of exposing rat COC to $500 \mathrm{ng} / \mathrm{mL}$ nicotine on GDF9 protein levels are depicted in Figure 9-11. Multiple bands between 20 and $114 \mathrm{kDa}$ were detected in the positive control of both replicates. The bands in the positive control of the first replicate (Figure 9-11A) were overexposed, so most individual bands were indistinguishable. Bands of 49 and $20 \mathrm{kDa}$ in size, corresponding to the promature and mature forms of GDF9 respectively were detected in the positive control lanes. Additional prominent bands of 93, 70, and $40 \mathrm{kDa}$ were observed. No bands were observed in the negative control. When the GDF9 antibody was pre-absorbed with recombinant GDF9, no bands were detected (Figure 9-11C). Multiple bands were observed in each of the lanes containing COC lysate, corresponding to the aforementioned molecular weights in the positive control.

In the first replicate experiment (Figure 9-11A), promature GDF9 levels did not change over time in the control group. Incubation of $\mathrm{COC}$ for 1 hour with nicotine resulted in promature GDF9 levels 2.5-fold lower than that in the control. However, promature GDF9 levels increased 3.3-fold with time in the nicotine-treated group such that levels were similar to that in control COC at 25 hours. Mature GDF9 was detected in the control COC after 1 hour of incubation, but the levels declined 1.7-fold with time. Mature GDF9 was undetectable in the nicotine-treated $\mathrm{COC}$ at 1 hour in culture but had increased by 25 hours in culture to levels nearly twice that of control COC. 
Similar to the first replicate, promature GDF9 levels in the second replicate experiment did not change over time in the control group (Figure 9-11B). Levels of promature GDF9 were 1.3-fold greater in the nicotine-treated group at 1 hour compared to the control, however decreased 1.2-fold with time to levels similar to that in the control at 25 hours. Mature GDF9 was undetectable at all time points regardless of treatment.

Overall, due to the large difference between replicates in the promature GDF9 levels in the nicotine-treated group at the 1 hour time point, no effect of time or treatment was observed for promature GDF9 levels (Figure 9-12). 
A

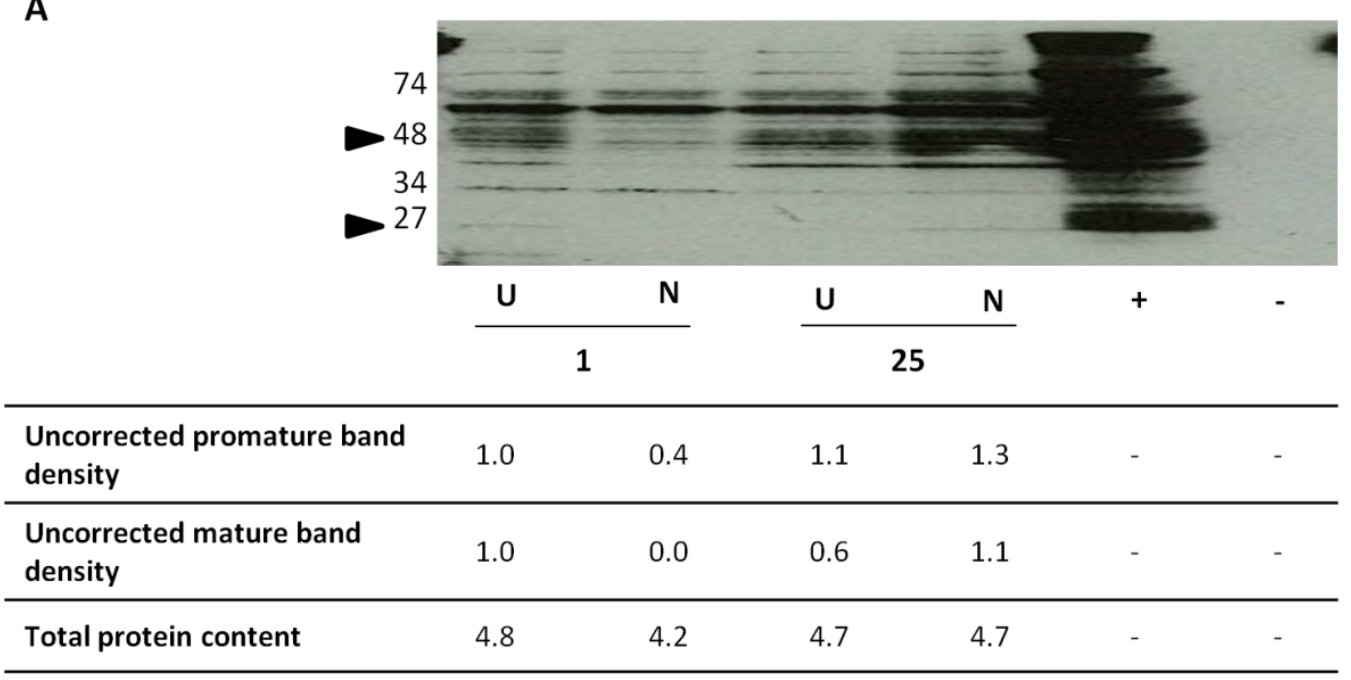

B

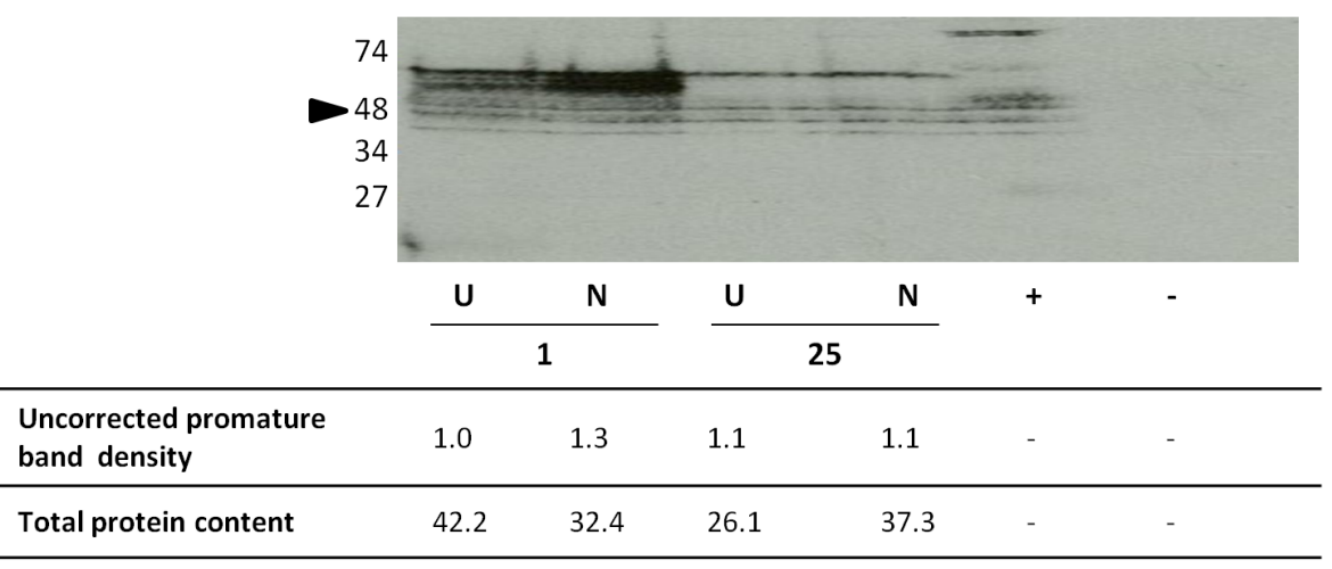

C

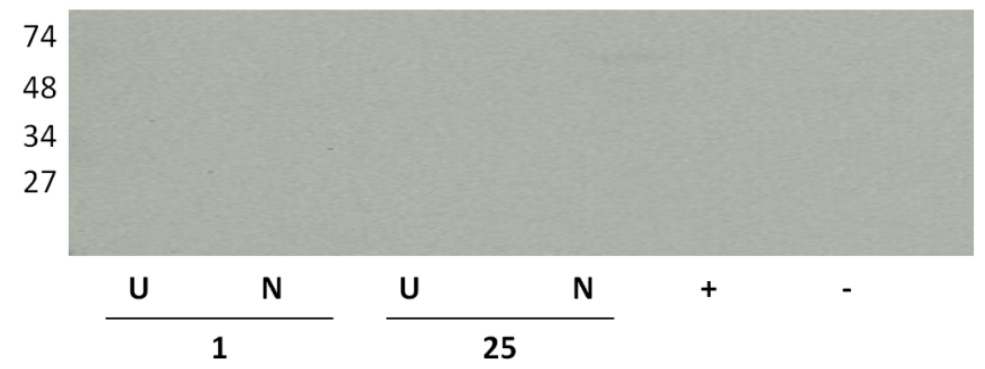

Figure 9-11: The effects of exposing rat COC to nicotine in vitro on GDF9 protein levels over time. Western blots were performed in duplicate (A and B) to determine the level of GDF9 protein in COC lysate. COC were either incubated for 1 or 25 hours with the solvent control (U) or nicotine (N). Rat ovary lysate was included as a positive control (+) and E. coli produced recombinant ovine BMP15 was included as a negative control (-). A control experiment (C) was also run where the blot shown in (B) was stripped and re-probed with the GDF9specific antibody that had been pre-absorbed with E. coli produced recombinant ovine GDF9. Molecular sizes $(\mathrm{kDa})$ are indicated to the left of each blot. The densities of the bands of interest (indicated by arrowheads) were measured and corrected for the control at 1 hour. Total protein $(\mu \mathrm{g})$ in each sample was determined using the BCA assay. 


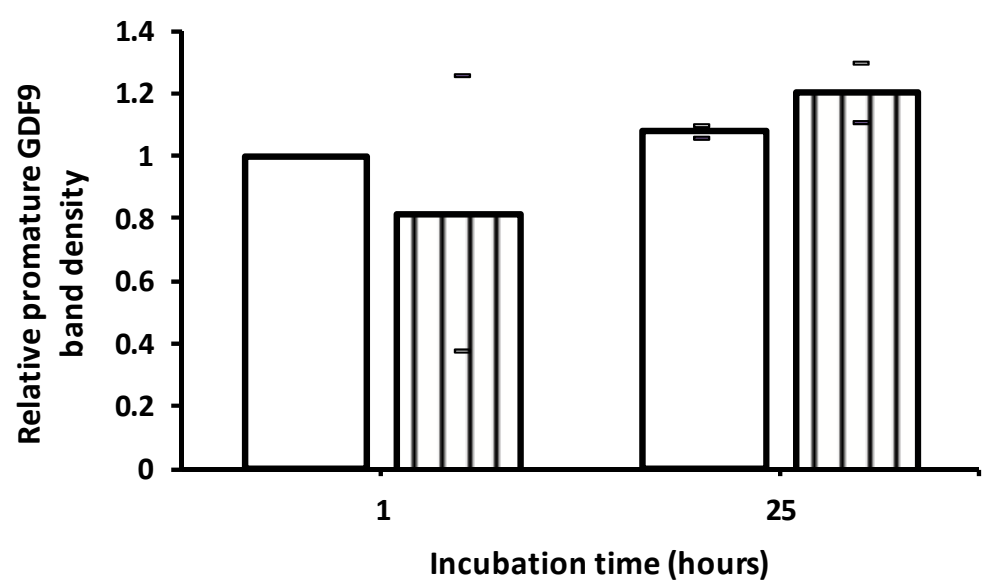

Figure 9-12: Effect of nicotine on relative GDF9 levels in rat COC. The control group is represented by the unfilled bars ( $\square$ ), while the nicotine-treated group is represented by the striped bars ( $\square$ ). The bars represent the means, while the dashes represent each individual band density measurement.

\subsubsection{Effect of MDMA and THC on protein levels in rat COC}

The effects of exposing rat COC to $2 \mu \mathrm{g} / \mathrm{mL}$ MDMA and $500 \mathrm{ng} / \mathrm{mL}$ THC on CX43 protein levels are depicted in Figure 9-13. A $43 \mathrm{kDa}$ band was observed in the positive control of both experimental replicates, while no bands were observed in the negative control of either replicate. A $43 \mathrm{kDa}$ band was observed in each of the lanes containing COC lysate. When the CX43 antibody was pre-absorbed with the control peptide, no bands were detected (Figure $9-13 C)$.

In the first replicate experiment (Figure 9-13A), CX43 levels decreased 2.5-fold with time in the control COC. Similarly, the levels of CX43 also decreased 3.3-fold with time in the THCtreated COC, so that levels were similar to the controls at both times. In contrast, CX43 levels were 1.25-fold lower in the MDMA-treated COC at 1 hour compared to the control, and did not change with time so that the levels were 1.75 -fold greater than that in the control COC at 25 hours.

In the second replicate experiment, levels of CX43 decreased 3.3-fold with time in the control COC (Figure 9-13B). CX43 levels were similar between experimental groups after 1 hour of culture time, and a reduction in CX43 levels over time was noted in all groups (Figure 9-13B). Levels of CX43 decreased 2-fold and 1.2-fold with time in the MDMA- and THCtreated COC respectively. The CX43 levels in COC incubated with MDMA and THC for 25 hours were 1.7-fold and 3-fold greater respectively compared to the control. 
A consistent trend of a decrease in CX43 levels over time was observed in all experimental groups but this was more evident in the control group (Figure 9-14). The smaller decreases in the MDMA- and THC-treated group resulted in CX43 levels being higher in the treated COC, compared to the control, after 25 hours in culture. 
A

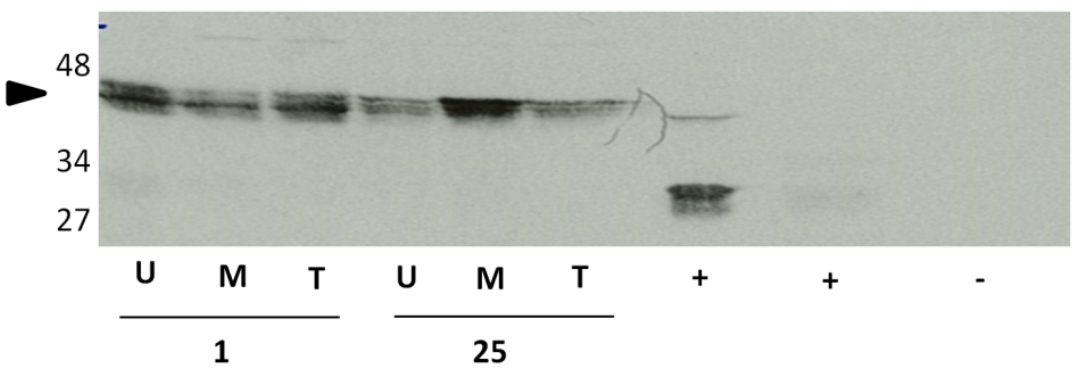

\begin{tabular}{lccccccccc}
\hline $\begin{array}{l}\text { Uncorrected } \\
\text { band density }\end{array}$ & 1.0 & 0.6 & 0.9 & 0.4 & 1.1 & 0.4 & - & - & - \\
\hline $\begin{array}{l}\text { Total protein } \\
\text { content }\end{array}$ & 2.8 & 2.1 & 2.6 & 3.4 & 4.2 & 4.0 & - & - & - \\
\hline $\begin{array}{l}\text { Band density/ } \\
\text { protein content }\end{array}$ & 1.0 & 0.8 & 1.0 & 0.4 & 0.7 & 0.3 & - & - & - \\
\hline
\end{tabular}

B

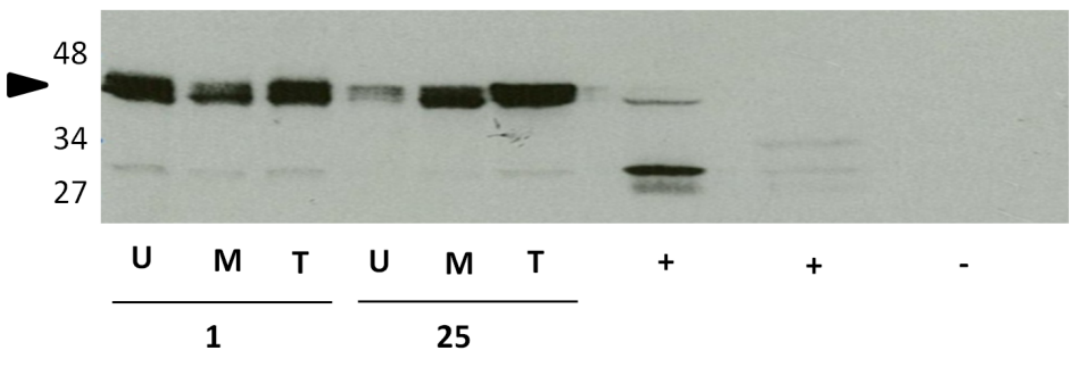

\begin{tabular}{llllllllll}
\hline $\begin{array}{l}\text { Uncorrected } \\
\text { band density }\end{array}$ & 1.0 & 0.7 & 0.9 & 0.2 & 0.8 & 0.9 & - & - & - \\
\hline $\begin{array}{l}\text { Total protein } \\
\text { content }\end{array}$ & 19.9 & 13.5 & 16.4 & 17.8 & 31.8 & 21.2 & - & - & - \\
\hline $\begin{array}{l}\text { Band density/ } \\
\text { protein content }\end{array}$ & 1.0 & 1.0 & 1.1 & 0.3 & 0.5 & 0.9 & - & - & - \\
\hline
\end{tabular}

C

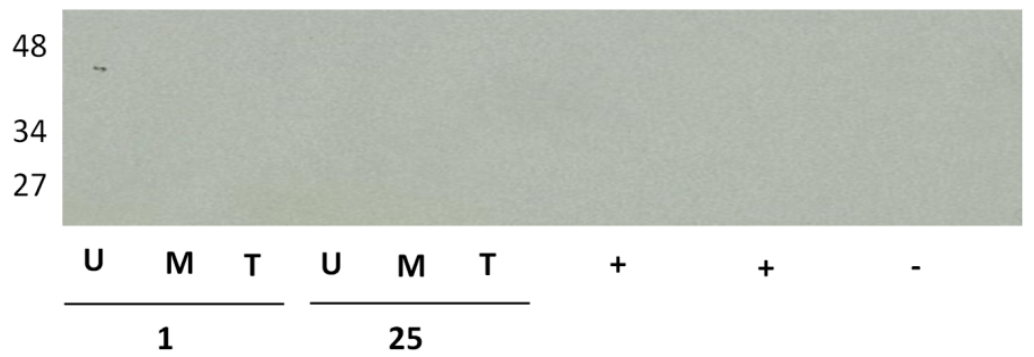

Figure 9-13: The effects of exposing rat COC to MDMA and THC in vitro on CX43 protein levels over time. Western blots were performed in duplicate $(A$ and $B$ ) to determine the level of $C X 43$ protein in $C O C$ lysate. $C O C$ were either incubated for 1 or 25 hours with the solvent control (U), MDMA (M) or THC (T). Rat ovary lysate was included as a positive control $(+)$ and $E$. coli produced recombinant ovine BMP15 was included as a negative control (-). A control experiment (C) was also performed where the blot shown in (B) was stripped and re-probed with the CX43-specific antibody that had been pre-absorbed with its control peptide. Molecular sizes $(\mathrm{kDa})$ are indicated to the left of each blot. The density of the band of interest (indicated by an arrowhead) was measured and corrected for the control at 1 hour. Total protein $(\mu \mathrm{g})$ in each sample was determined using the $\mathrm{BCA}$ assay. The relative band densities were corrected for total protein amount, as calculated by the BCA assay. 


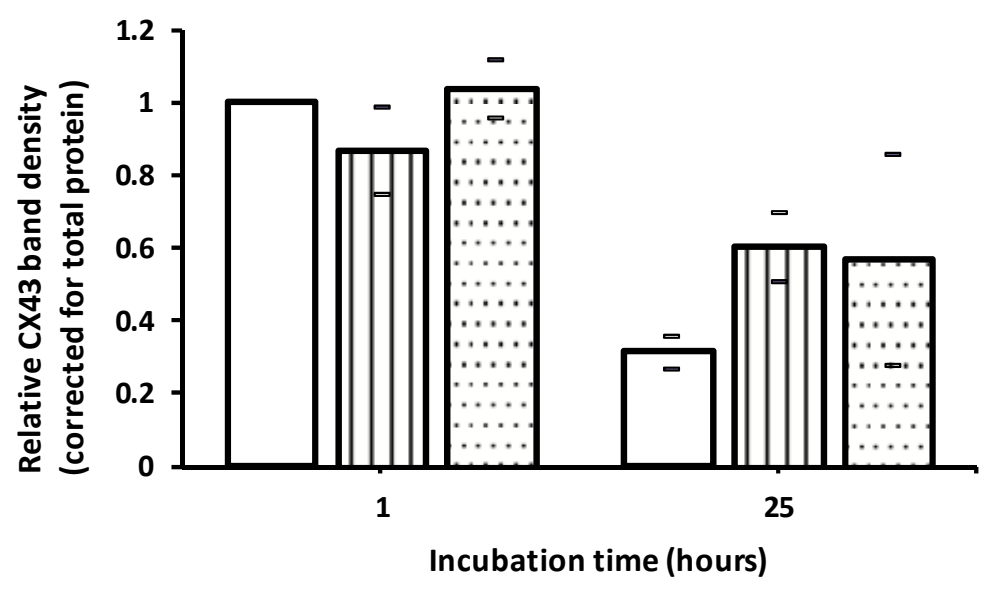

Figure 9-14: Effect of MDMA and THC on relative CX43 levels in rat COC. The control group is represented by the unfilled bars ( $\square$ ), the MDMA-treated group is represented by the striped bars ( $\square$ ), while the THCtreated group is represented by the dotted bars $(\cdots)$. The bars represent the means, while the dashes represent each individual band density measurement.

The effects of exposing of rat COC to $2 \mu \mathrm{g} / \mathrm{mL}$ MDMA and $500 \mathrm{ng} / \mathrm{mL}$ THC on GDF9 protein levels are depicted in Figure 9-15. Multiple bands between 20 and 114 kDa were detected in the positive control of both replicates. Bands of 49 and $20 \mathrm{kDa}$ in size, corresponding to the promature and mature forms of GDF9 respectively were detected in the positive control lanes. Additional prominent bands of 93,70 , and $40 \mathrm{kDa}$ were observed. No bands were observed in the negative control. When the GDF9 antibody was pre-absorbed with recombinant GDF9, no bands were detected (Figure 9-11C). Multiple bands were observed in each of the lanes containing COC lysate, corresponding to the aforementioned molecular weights in the positive control.

In the first replicate experiment (Figure 9-15A), levels of promature GDF9 decreased 1.25fold with time in the control group. Levels of promature GDF9 were similar after 1 hour in culture in all experimental groups. Promature GDF9 levels increased slightly (1.4-fold and 1.2-fold) with time in the MDMA- and THC-treated COC to levels 1.6 -fold greater in the MDMA- and THC-treated compared to the control COC at 25 hours. Mature GDF9 was detected at every time point regardless of treatment. Mature GDF9 levels declined 2.5-fold with time in the control COC. Following incubation with MDMA or THC for 1 hour, mature GDF9 levels were 2.5-fold lower than that in the control. Mature GDF9 levels increased 2.75fold with time in the MDMA-treated COC so that levels were 2.8-fold greater than the 
control at 25 hours. Mature GDF9 levels remained constant in the THC-treated COC so that levels were similar to the control at 25 hours.

Similar to the first replicate, levels of promature GDF9 decreased 2-fold with time in the control group (Figure 9-15B). Promature GDF9 levels were 1.7-fold and 5-fold lower in the MDMA- and THC-treated COC compared to the control COC at 1 hour. Levels of promature GDF9 in COC incubated with MDMA or THC increased with time by 1.5 -fold and 6-fold respectively. This resulted in the levels of promature GDF9 being 1.8-fold and 2.4-fold higher in the MDMA- and THC-treated COC than the control COC at the 25 hour time point. Mature GDF9 levels were undetectable at any time point regardless of treatment.

The levels of promature GDF9 consistently decreased over time in the control group (Figure 9-16). Conversely, there was high variability in regards to the effect of treatment on the promature GDF9 levels after 1 hour in culture. However with time, both the MDMA and THC-treated COC showed an increase in promature GDF9 levels. 
A

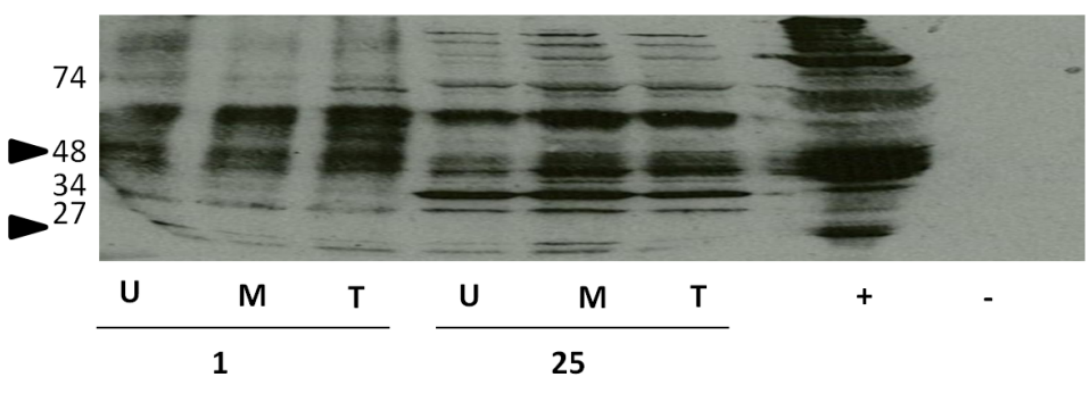

\begin{tabular}{lcccccccc}
\hline $\begin{array}{l}\text { Uncorrected promature } \\
\text { band density }\end{array}$ & 1.0 & 0.9 & 1.1 & 0.8 & 1.3 & 1.3 & - & - \\
\hline $\begin{array}{l}\text { Uncorrected mature band } \\
\text { density }\end{array}$ & 1.0 & 0.4 & 0.4 & 0.4 & 1.1 & 0.3 & - & - \\
\hline Total protein content & 5.2 & 5.1 & 5.1 & 5.5 & 5.3 & 5.3 & - & - \\
\hline
\end{tabular}

B

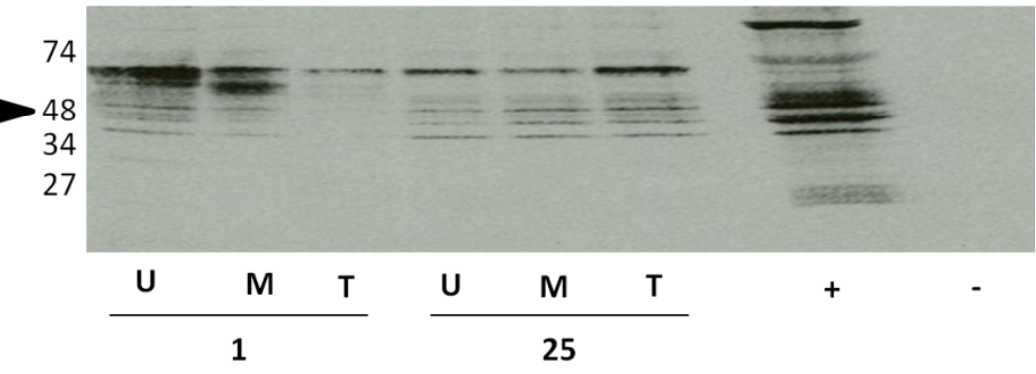

\begin{tabular}{lcccccccc}
\hline $\begin{array}{l}\text { Uncorrected promature } \\
\text { band density }\end{array}$ & 1.0 & 0.6 & 0.2 & 0.5 & 0.9 & 1.2 & - & - \\
\hline Total protein content & 4.9 & 4.2 & 3.3 & 3.7 & 4.9 & 5.6 & - & - \\
\hline
\end{tabular}

\section{C}

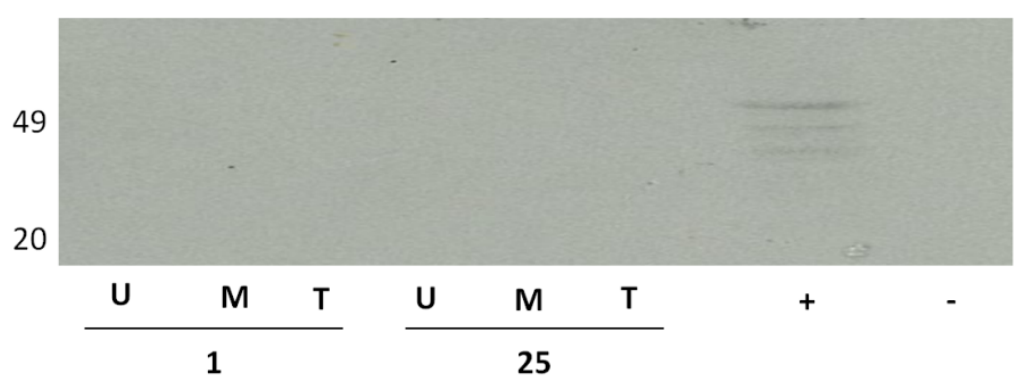

Figure 9-15: The effects of exposing rat COC to MDMA and THC in vitro on GDF9 protein levels over time. Western blots were performed in duplicate ( $A$ and $B$ ) to determine the level of GDF9 protein in COC lysate. COC were either incubated for 1 or 25 hours with the solvent control (U), MDMA (M) or THC (T). Rat ovary lysate was included as a positive control $(+)$ and $E$. coli produced recombinant ovine BMP15 was included as a negative control (-). A control experiment (C) was also performed where the blot shown in (B) was stripped and re-probed with the GDF9-specific antibody that had been pre-absorbed with $E$. coli produced recombinant ovine GDF9. Molecular sizes $(\mathrm{kDa})$ are indicated to the left of each blot. The densities of the bands of interest (indicated by arrowheads) were measured and corrected for the control at 1 hour. Total protein ( $\mu \mathrm{g}$ ) in each sample was determined using the BCA assay. 


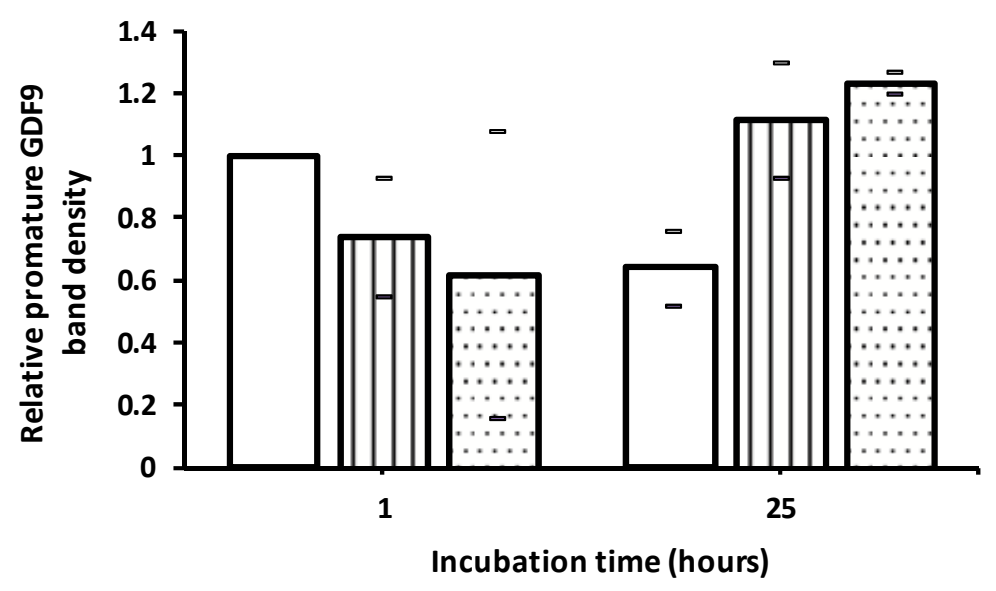

Figure 9-16: Effect of MDMA and THC on relative GDF9 levels in rat COC. The control group is represented by the unfilled bars ( $\square$ ), the MDMA-treated group is represented by the striped bars ( $\square$ ), while the THCtreated group is represented by the dotted bars $(\cdots)$. The bars represent the means, while the dashes represent each individual band density measurement.

\subsection{Discussion}

This study revealed a consistent pattern of CX43 protein expression in cultured rat $\mathrm{COC}$ with time that enabled the effects of short and long-term exposures to common lifestyle factors and environmental contaminants to be assessed. Overall, the CX43 protein levels were lower after rat $\mathrm{COC}$ were cultured for 25 hours, compared to that after 1 hour. However exposure of rat COC to nicotine, MDMA, and THC for 25 hours resulted in CX43 protein levels that were higher than in the appropriate controls. It was interesting to note that the CX43 levels were sometimes altered after a 1 hour exposure of rat $\mathrm{COC}$ to some compounds. Specifically, CX43 protein levels were enhanced following a 1 hour exposure to caffeine, and reduced after exposure to nicotine. The effect of time on GDF9 protein expression in cultured rat $\mathrm{COC}$ was variable across experiments, thereby confounding interpretations concerning the effects of treatments on protein expression levels. Nevertheless, the evidence did suggest that promature GDF9 levels were reduced following short-term in vitro exposures (i.e. 1 hour) to BPA and caffeine, but enhanced after longer exposures (i.e. 25 hours) to BPA, caffeine, MDMA, and THC.

CX43 levels in COC cultured with BPA were not different to the levels in the control group however there was variability between the replicates noted after a 1 hour exposure to BPA. In Chapter 6 it was established that BPA enhanced GJ communication between the CC and 
oocyte, particularly after 25 hours exposure. Therefore, it seems that the BPA-mediated upregulation of GJ communication does not occur through changes in CX43 protein expression. The findings from this study are consistent with a previous study which found that BPA did not affect CX43 levels in a rat epithelium derived mammary cell line (Lee and Rhee, 2007). Levels of both promature and mature GDF9 in COC were reduced after a 1 hour exposure to BPA, suggesting a reduction in oocyte growth factor signalling. However, after 25 hours exposure to BPA, promature and mature GDF9 levels were increased, indicating enhanced oocyte growth factor signalling. The finding that short and long term exposures to BPA have opposing effects on GDF9 levels suggest that desensitisation or metabolism of BPA occurs, or that two different regulatory mechanisms are at play in the COC. Given the role of GDF9 in regulating CC functions such as proliferation, metabolism, and apoptosis, further investigation into these pathways would provide insight into how BPA affects COC health.

Rat COC that were exposed to caffeine for 1 hour had increased CX43 levels compared to the control, however this effect was attenuated after a 25 hour exposure. Interestingly, in Chapter 6 it was determined that caffeine may enhance GJ communication ( $P=0.052)$, and that this effect was most notable after a 25 hour exposure. Therefore, as with BPA, these results suggest that changes in CX43 protein levels are not responsible for the increase in GJ communication that was seen in COC incubated with caffeine for 25 hours. Similar to the effect of BPA on GDF9 protein, levels of promature and mature GDF9 were reduced in COC incubated with caffeine for 1 hour, but were markedly higher than the controls following a 25 hour exposure to caffeine. Again, these findings suggest that metabolism or desensitization to caffeine occurs in the $\mathrm{COC}$, or that two different regulatory mechanisms are at play. The marked effects of caffeine on GDF9 protein levels indicates that CC functions mediated by oocyte-secreted factors may be altered following exposure to caffeine in vitro. Specifically, functions such as CC proliferation and metabolism that are enhanced by oocytesecreted factors may be reduced following a short exposure to caffeine, but increased following a long exposure. Further investigation into oocyte-mediated CC functions will reveal how caffeine affects these pathways.

CX43 levels in COC were not noticeably influenced by ethanol either after a 1 or 25 hour exposure. These results are consistent with those reported in Chapter 6, where it was shown that ethanol did not affect GJ communication in COC. A previous study found markedly 
reduced levels of CX43 protein in rat liver epithelial cells that had been exposed to ethanol for 24 hours (Bokkala et al., 2001). Interestingly, in that study CX43 protein levels were also correlated with a reduction in GJ communication. In the current study, a doublet band was observed in both replicates, indicating CX43 was differentially phosphorylated (Solan and Lampe, 2009). However, no consistent pattern of phosphorylation was observed. It should be noted that no effect of culture time on CX43 was noted in the control group of the ethanol experiment. In all other experiments, CX43 levels declined markedly over time in the control group. The reason for this is unknown, however the solvent control used for ethanol, was $\mathrm{dH}_{2} \mathrm{O}$, in contrast to the use of DMSO as the solvent control for all other experiments. Exposure to ethanol did not affect levels of promature GDF9, and the mature protein was not detected in either experiment.

CX43 levels in nicotine-treated COC were markedly reduced at 1 hour, whereas were increased at $\mathbf{2 5}$ hours, compared to the control. The variability that was noted in the effect of nicotine over time suggests that metabolism or desensitisation to nicotine occurs in the COC. The effect of nicotine on CX43 protein levels was not dissimilar to the pattern of GJ communication in $\mathrm{COC}$ following incubation with nicotine (Chapter 6). GJ communication in COC was unaffected by a 1 hour exposure to nicotine, however it was increased after a 25 hour incubation. These results suggest that the increase in GJ communication in COC following long term exposure to nicotine is mediated by a concomitant increase in CX43 protein levels. However, the reduction in CX43 protein following a 1 hour exposure of COC to nicotine was not observed to influence GJ communication in COC (Chapter 6). Furthermore, the finding that the reduction in CX43 levels over time in the control that was not seen in the nicotine-treated COC indicates that nicotine may, in some manner, prevent the degradation of CX43 protein over time. Interestingly, the results presented in this chapter are in contrast to a previous study which showed that CX43 levels were unaffected by short exposures to nicotine (between 0 and 8 hours) but that long exposures (24 hours) decreased CX43 in human umbilical vein endothelial cells, (Tsai et al., 2004). In the present study, doublet bands were observed in all samples, indicating differentially phosphorylated forms of CX43 are present. The slow migrating form of CX43 likely corresponds to a phosphorylated form (Solan and Lampe, 2009) and generally displayed a reduced intensity compared to the faster migrating isoform. Exposure to nicotine in vitro did not affect levels of GDF9 protein in COC. 
CX43 and GDF9 levels in COC that were incubated with MDMA for 25 hours were increased slightly compared to the control group. However, a short exposure time (i.e. 1 hour) was without effect. The levels of CX43 protein noted here do not correlate to the pattern of GJ communication in COC following incubation with MDMA (Chapter 6). Indeed, no effect of MDMA on GJ communication was measured. Therefore, while MDMA may increase overall CX43 levels, these connexins may not form functional GJ channels. Furthermore, the finding that MDMA partially prevented the time-dependent decrease in CX43 levels that was seen in the control suggests that MDMA prevents CX43 degradation. The results presented in this chapter are in contrast to a previous study in which MDMA reduced CX43 levels in rat ventricular myocytes following a 1 hour exposure (Zhuo et al., 2013). The effect of MDMA on GDF9 has not been investigated previously, to the best of my knowledge. These results suggest that long-term exposures of COC to MDMA are likely to modulate oocyte-CC interactions and thereby modulate CC functions such as metabolism and proliferation.

Exposure to THC did not affect CX43 levels in rat COC. These results correlate with those from Chapter 6, whereby incubation of COC with THC in vitro did not affect GJ communication. Conversely, incubation of $\mathrm{COC}$ with THC for 25 hours resulted in increased levels of promature GDF9 compared to the control. Mature GDF9 was only detected in the first replicate experiments however these preliminary results suggest that mature GDF9 was reduced after a 1 hour exposure to THC. Taken together, these results demonstrate that long exposures to THC may influence oocyte-secreted factor production, and thus oocytemediated CC functions such as proliferation or metabolism.

In general, these protein expression studies gave less reliable results than the gene expression or GJ communication studies presented in Chapters 6, 7, and 8. Therefore, confirmation of these preliminary results by additional replicate experiments is necessary. The variation observed in the Western blot results is likely attributable to the Western blotting technique used and that only two replicate experiments were carried out due to challenge in undertaking several replicate experiments. Small differences in Western transfer efficiency, primary and/or secondary antibody concentrations, and developing reagents can result in large differences in band density. A commonly used approach to compensate for differences in protein loading is to correct band densities for a reference protein such as GADPH (Ratchford et al., 2008; Wang et al., 2009). In this study the blots 
were stripped and re-probed three times (i.e. for CX43, GDF9, and the two pre-absorbed antibody controls). As repeated stripping of the blot can remove protein in addition to the bound antibodies, a reference protein was omitted from this study and instead the total protein content was determined using the BCA assay. Moreover, a constant number of COC (i.e. 200) were included in each group. However, these methods are limited in that they do not account for loading error or differences in protein transfer (Aldridge et al., 2008).

The total protein content of each sample was expected to be influenced mainly by the number of CC. Therefore, the levels of CC-derived CX43 protein was corrected for total protein amount. The amount of protein calculated in each of the samples was extremely variable between experiments, despite using the same number of $\mathrm{COC}$ in each. An adapted BCA assay protocol was utilised for some experiments, where the plates were incubated at $60{ }^{\circ} \mathrm{C}$ rather than $37{ }^{\circ} \mathrm{C}$. This provided much greater sensitivity and allowed for a more accurate detection of protein content however this may have contributed to the variability seen between experiments. Since the total protein content was used relative to the other samples in each experiment, and was not used to compare samples across experiments, this should not have affected interpretation of the results. In contrast to the protein levels for CX43, those for GDF9 were not corrected for total protein as the relative band densities were produced by a known number of oocytes.

The CX43-specific antibody, MAB3067, used in this study was relatively specific. However, additional, unidentified 24 and $68 \mathrm{kDa}$ bands were seen in some experiments that were not apparent when the antibody was pre-absorbed with the control peptide. In some experiments, differentially phosphorylated forms of CX43 were identified as doublet bands (Solan and Lampe, 2009) however these doublet bands were not always resolved. Preliminary testing of an antibody specific for CX43 phosphorylated on serine residues 279/282 (sc12900; Santa Cruz Biotechnology, USA) was undertaken during these studies. Phosphorylation of these residues is involved in the MAPK-dependent reduction in GJ communication following the LH surge (Norris et al., 2008). Furthermore, an antibody specific to CX37 was also tested for the measurement of CX37 in rat COC (CX37B12-A; Alpha Diagnostic, USA). However, the levels of CX37 and these phosphorylated forms of CX43 were extremely low in rat $\mathrm{COC}$. Hence given the challenge in generating large numbers of $\mathrm{COC}$ for 
protein measurements it was considered to be impractical to include these proteins in the current study.

The GDF9-specific antibody, MAB47B, used in this study was produced by Oxford Brooks University for AgResearch. A large number of bands were detected using this antibody. It is likely that the band or set of bands around $49 \mathrm{kDa}$ in size represent uncleaved promature GDF9 while the 20 kDa band likely represents mature GDF9 (Lin et al., 2012). It is possible that some of the other bands observed were differentially processed forms of GDF9 such as the preproprotein and glycosylated and/or phosphorylated forms. It is important to note that that immunoneutralisation of GDF9 using MAB47B blocked the ability of rat oocytes to stimulate rat GC proliferation (Lin et al., 2012), demonstrating that MAB47B recognises bioactive GDF9. When the monoclonal antibody was pre-absorbed with recombinant ovine GDF9, none of the aforementioned bands were detected. This indicated that the antibody is specific for GDF9 and is capable of recognising multiple forms although this test alone does not confirm complete antibody specificity (Swaab et al., 1977). Thus, there is a possibility that some of the bands detected with MAB47B are due to non-specific binding. On the other hand, the antibody was probing both secreted forms of GDF9 and intracellular protein that were being synthesized and processed. Thus, it is perhaps not surprising that multiple forms were present. In this context, a similar caveat can be applied to the measure of the forms of CX43. In the present study, the promature form of GDF9 was in higher abundance compared to mature GDF9, however at the present time the bioactivity of the promature protein form relative to the mature form of GDF9 is unknown. In addition to the GDF9-specific antibody that was used, several BMP15-specific antibodies were tested for application in this study (H-83 and A-20, Santa Cruz Biotechnology; MAB61a, produced by Oxford Brookes University for AgResearch). However, consistent with a previous study also on rat COC (Lin et al., 2012), the levels of BMP15 protein were very low and therefore, measurement of this protein was not undertaken any further. In summary, this chapter demonstrates that short and long term effects of lifestyle factors on CC-derived CX43 protein levels were detected using Western blotting. Levels of $\mathrm{CX} 43$ protein following a short-term exposure to caffeine were increased, but to nicotine were reduced. Furthermore, a long-term exposure to nicotine or MDMA increased CX43 protein in rat COC. The use of Western blotting to determine the effects of lifestyle on oocyte-derived GDF9 protein levels was less definitive due to inconsistencies 
between the patterns of GDF9 expression in the control groups. Nevertheless, levels of GDF9 were reduced following short-term exposure to BPA and caffeine, but were increased following long-term exposure to BPA, caffeine, MDMA, or THC. It is important to note that these protein expression studies provide preliminary results, due to the limited number of replicates, and that these results need to be confirmed. 


\section{EFFECTS OF IN VIVO EXPOSURE TO MDMA ON COC FUNCTION}

\subsection{Introduction}

In vitro studies are invaluable for testing the effects of specific compounds on isolated cells however these compounds may elicit different effects on the same cells in vivo. This difference in responsiveness is due to many variables such as the route of access to the blood stream, half-life of the compound in the circulation, transit efficiency to the target cells and rate of peripheral metabolism. MDMA (ecstasy) is an amphetamine derivative that is primarily used by young people (Schenk, 2009). Acute physiological effects of MDMA include euphoria, increased energy levels, increased heart rate and blood pressure, chills, sweating, tremor, and muscle tension (Easton and Marsden, 2006). While the use of MDMA is typically infrequent, $35 \%$ of subjects interviewed in an Australian study had binged on ecstasy in the past 6 months, with the median length of a binge around 3 days (Topp et al., 1999). In humans and rats, first pass metabolism of MDMA includes $N$-demethylation that produces 3,4-methylenedioxyamphetamine (MDA), and demethylation which produces 3,4dihydroxymethamphetamine (HHMA) (Green et al., 2003). In vivo, these products are further processed into 12 additional metabolites which have varying biological activities.

There is little available information regarding the effect of MDMA on fertility and reproductive health in either males or females. Both acute and chronic exposure to MDMA perturbed the gonadal-hypothalamic axis in male rats resulting in reduced levels of hypothalamic GnRH mRNA expression and serum testosterone (Dickerson et al., 2008). Chronic exposure to MDMA in utero in rats was found to disrupt the fertility of male offspring, through elevated levels of DNA damage in sperm, increased interstitial oedema, and decreased sperm counts and motility (Barenys et al., 2010). Similarly, adult male rats that were chronically exposed to MDMA also had increased DNA damage in sperm, tubular degeneration, and interstitial oedema in the testes, however sperm motility, morphology and ability to induce pregnancy were unaffected (Barenys et al., 2009). In the current study it was established that in vitro exposure of rat COC to MDMA affected CC function through altered levels of $C_{x} 43, C y c s$, Gfpt1, and Pfkp mRNA (Chapter 8) and CX43 protein (Chapter 9). 
The aim of this chapter was to investigate the effects of in vivo exposure of female rats to MDMA on COC health. Specifically, the levels of CC-derived Atr, Cx43, Cycs, Gfpt1 and Pfkp mRNA, and oocyte-derived Atr, Bmp15, Cx37 and Gdf9 mRNA were measured. In addition, the effects of in vivo exposure to MDMA on protein levels of CX43 and GDF9 in rat COC were determined.

\subsection{Methods}

Pre-pubescent female rats ( $\mathrm{N}=12 /$ group) were administered $5 \mathrm{mg} / \mathrm{kg} /$ day of $\mathrm{MDMA}$ (treated) or the vehicle (saline; control) alone via ip injection once per day for three days. The rats were habitualised in locomotor activity boxes for 30 minutes prior to receiving the injection. After the injections were performed, the rats were returned to the locomotor activity boxes for 60 minutes thereafter. Whilst in the locomotor activity boxes, the ambulatory counts for each rat were measured at 5 minute intervals as described in Section 2.2.1. Approximately 18 hours after the final injection, the rats were sacrificed and the ovaries were collected in sterile PBS. The COC from rats in the same treatment group were extracted and pooled. The COC were subsequently collected into PBS for either qPCR or Western blotting.

The COC that were collected for qPCR were transferred individually into a 96-well plate containing $10 \mu \mathrm{L}$ PBS, where the CC were stripped from the oocyte as described previously. The oocyte was washed twice in PBS, before being transferred into $10 \mu \mathrm{L}$ PBS. The 96-well plates containing the DO and $\mathrm{CC}$ were stored at $-80^{\circ} \mathrm{C}$. Total RNA was extracted from the DO and CC, and was synthesised into cDNA. Multiplex TaqMan qPCR was used to measure the mRNA levels of the following candidate genes: Gdf9, Bmp15, and $C \times 37$ in oocytes; $C \times 43$, Gfpt1, and Pfkp in CC; and Atr, Cycs, Glut1 in oocytes and CC. The reference gene Rpl19 was included in each reaction set to correct for cDNA template input. A calibrator sample was included in every run to correct for between-run variation. Those samples which had an $R p / 19 C_{T}$ value $>30$ were excluded from the analysis due to insufficient mRNA levels. For each oocyte gene within each sample, the $C_{T}$ value was corrected for the $C_{T}$ value of the calibrator sample from the appropriate run. For each $\mathrm{CC}$ gene within each sample, the $\mathrm{C}_{\mathrm{T}}$ value was corrected for the $R p / 19 C_{T}$ value from the same sample. The data was subsequently analysed 
by the $2^{(-\Delta \Delta C T)}$ method (Livak and Schmittgen, 2001). The data were examined for normality using the D'Agostino \& Pearson test and thereafter subjected to Mann Whitney analyses.

The COC that were collected for Western blotting were transferred into a microcentrifuge tube (200 COC/tube) and additional PBS was added to make the volume up to $500 \mu \mathrm{L}$. The $\mathrm{COC}$ were centrifuged at $800 \times \mathrm{g}$ for 10 minutes, the supernatant was removed, and the COC were resuspended in PBS supplemented with protease inhibitors. The samples were stored at $-20^{\circ} \mathrm{C}$ until further processing.

One-third of the volume of each sample ( 66 COC) was analysed for total protein amount using the BCA assay. The proteins in the remaining two-thirds of each sample were separated by size using SDS-PAGE and transferred onto a membrane. Western blotting procedures were then performed to detect and quantify CX43 and GDF9 protein expression. To determine band specificity, the blot was stripped and incubated with either the CX43 or GDF9 antibodies that had been pre-absorbed with the MAB3067 control peptide, or E. coli produced recombinant ovine GDF9 respectively. The density of each protein band was analysed using ImageJ software. For the CX43 blots, the density of the $43 \mathrm{kDa}$ band was measured. For the GDF9 blots, the densities of the $49 \mathrm{kDa}$ promature and, when present, the $20 \mathrm{kDa}$ mature bands were measured. When multiple bands were present (e.g. when doublet or triplet bands were present for $\mathrm{CX} 43$ ) the density of all bands was measured. The band densities for $\mathrm{CX} 43$ were corrected for the total protein present in the sample, as determined by the BCA assay. The band densities for GDF9 were uncorrected to give total GDF9 produced by a known number of oocytes.

\subsection{Results}

The effect of administering three doses of $5 \mathrm{mg} / \mathrm{kg}$ MDMA, each 24 hours apart, on ambulatory count before and after each injection in female rats is depicted in Figure 10-1. The mean ambulatory count began relatively high in both the treated and control groups during the habitualisation period, but this declined to baseline levels before injection of MDMA or saline at Time 0 . Following MDMA or saline administration, the mean ambulatory counts were initially raised in both groups, but the counts for the control group declined rapidly to baseline levels. In the MDMA-treated group, the mean ambulatory count rose steadily, peaking at 20-25 minutes post injection. The mean ambulatory count began to 
decline thereafter, but did not reach baseline levels before the end of the measurement period.

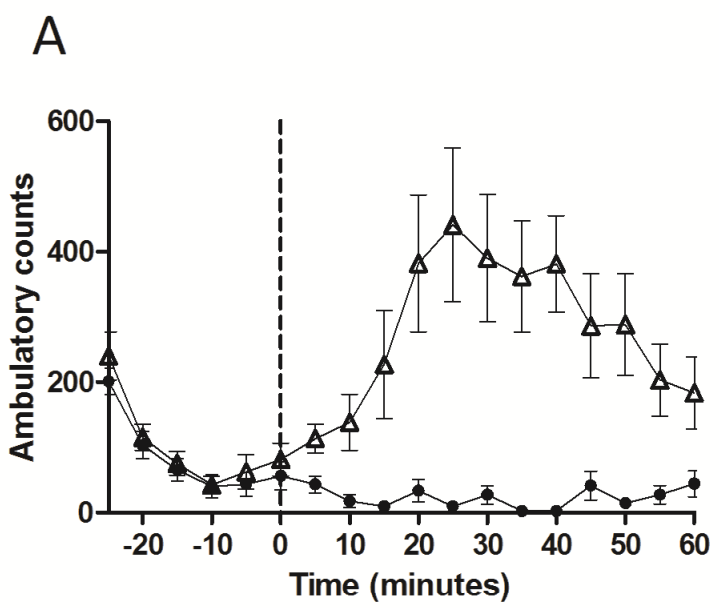

B

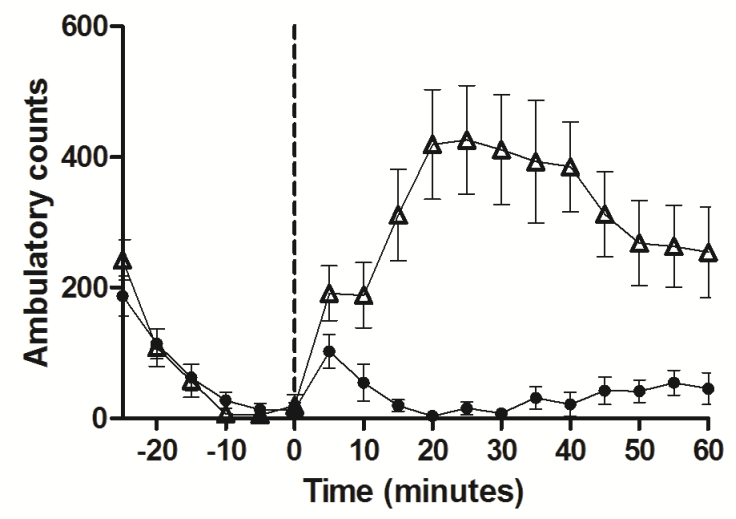

C

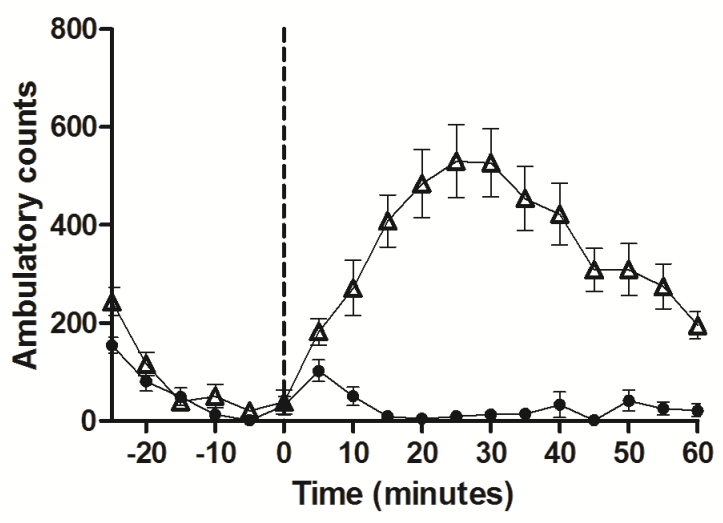

Figure 10-1: Effects of MDMA on ambulatory counts in female rats. The average ambulatory counts before and after the first injection (A), second injection (B), and third injection (C). Values represent the mean \pm SEM from 12 individuals. The control group is represented by the filled circles $\left({ }^{-}-\right)$while the MDMA-treated group is represented by the unfilled triangles $(\triangle)$. 
The effect of administering MDMA or the vehicle alone to female rats on meiotic arrest in COC is depicted in (Table 10-1). There was no difference in the proportion of oocytes that underwent GVBD between the control and MDMA-treated groups.

Table 10-1: Effect of MDMA on the proportion of oocytes with a visible GV in female rats. The table shows the proportion of oocytes with a visible GV (\%), with the actual number of oocytes with a GV in brackets. The data ( $\mathrm{N}=12$ oocytes/group from 12 rats) were analysed by a Fisher's exact test.

\begin{tabular}{cc}
\hline Control & MDMA \\
\hline $83(10)$ & $92(11)$ \\
\hline
\end{tabular}

The effect of administering MDMA or the vehicle alone to female rats on candidate gene expression in CC and DO is depicted in Figure 10-2. There were no differences in mRNA levels of any of the genes between the control and MDMA-treated groups. 
A

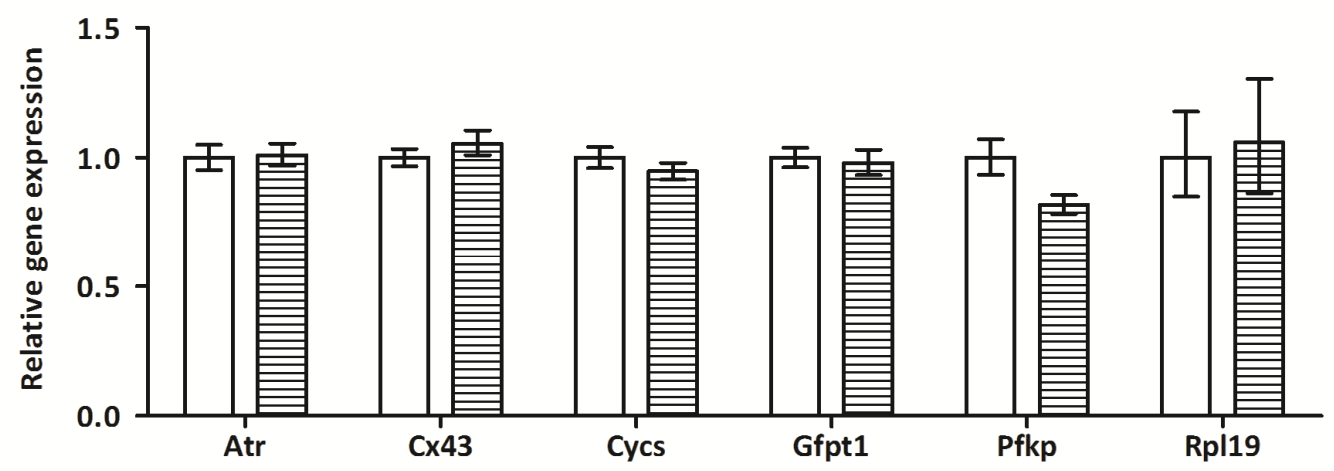

B

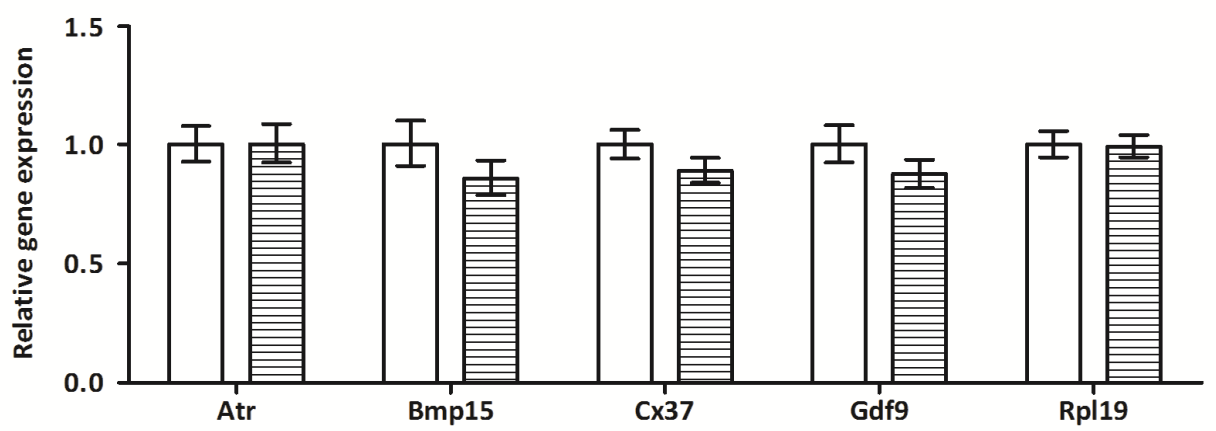

Figure 10-2: The effect of MDMA on candidate gene expression in CC (A) and oocytes (B) in female rats. The control group is represented by the unfilled bars ( $\square$ ), while the BPA treated group is represented by the hatched bars ( the mean of the control group. The data were generated from a total of 12 readings from individual oocytes or CC masses in each group. A Mann-Whitney test was performed for each gene.

The effect of administering MDMA or the vehicle alone to female rats on CX43 protein levels in $\mathrm{COC}$ is depicted in Figure 10-3. A $43 \mathrm{kDa}$ band was observed in the positive control of both experimental replicates, along with two smaller bands. No bands were observed in the negative controls of either replicate experiment. A $43 \mathrm{kDa}$ band was observed in both of the lanes containing COC lysate, along with one smaller band. The bands in the COC lanes in the second replicate (Figure 10-3B) were overexposed, so a shorter exposure time was undertaken to visualise the bands better (Figure 10-3C). When the CX43 antibody was preabsorbed with the control peptide, no bands were observed (Figure 10-3C).

Quantified CX43 protein levels in COC of rats administered MDMA or the vehicle alone are depicted in Figure 10-3. A double band of $43 \mathrm{kDa}$ in size is observed. The protein that migrated faster (i.e. the bottom band) is assumed to be the non-phosphorylated form to 
which the antibody is produced against, and the slow-migratory band (i.e. top band) which is assumed to be a phosphorylated form. In the first replicate, CX43 protein levels were 5-fold higher in COC of MDMA-treated, compared to control rats. A similar result was observed in the second replicate where CX43 protein levels were 2.7-fold higher in COC of MDMAtreated, compared to control, rats. Overall, CX43 protein levels were increased in COC from MDMA-treated rats, compared to the control (Figure 10-4). 
A

\begin{tabular}{llllll}
\hline & \\
& $\mathrm{U}$ & & \\
& & & \\
\end{tabular}

B

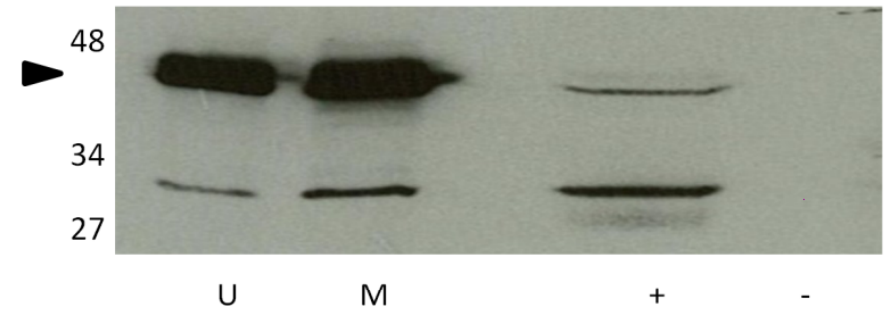

C

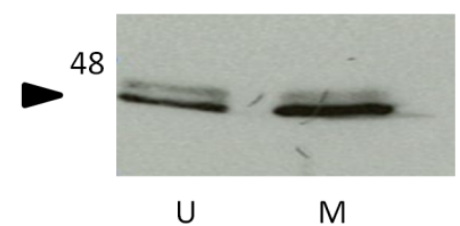

\begin{tabular}{lcccc}
\hline $\begin{array}{l}\text { Uncorrected band } \\
\text { density }\end{array}$ & 1.0 & 1.6 & - & - \\
\hline Total protein content & 2.5 & 1.5 & - & - \\
\hline $\begin{array}{l}\text { Band density/ } \\
\text { protein content }\end{array}$ & 1.0 & 2.7 & - & - \\
\hline
\end{tabular}

D

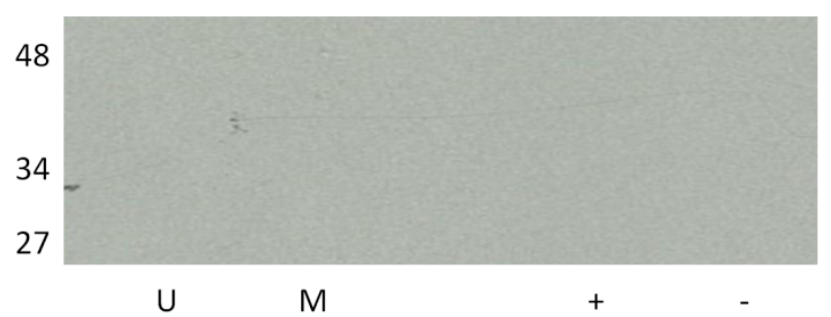

Figure 10-3: The effect of MDMA on CX43 protein levels in COC from female rats. Western blots were performed in duplicate ( $A$ and $B$ ) to determine the level of CX43 protein in COC lysate. COC were collected from rats injected with either the vehicle control (U), or $5 \mathrm{mg} / \mathrm{kg} /$ day MDMA (M). Rat ovary lysate was included as a positive control (+) and E. coli produced recombinant ovine BMP15 was included as a negative control (-). The second replicate (B) was developed for a shorter amount of time to allow visualisation of the individual bands (C). A control experiment (D) was also performed where the blot shown in (B) was stripped and re-probed with the CX43-specific antibody that had been pre-absorbed with its control peptide. Molecular sizes (kDa) are indicated to the left of each blot. The density of the band of interest (indicated by an arrowhead) was measured and corrected for the control at 1 hour. Total protein ( $\mu \mathrm{g}$ ) in each sample was determined using the BCA assay. The relative band densities were corrected for total protein amount, as calculated by the BCA assay. 


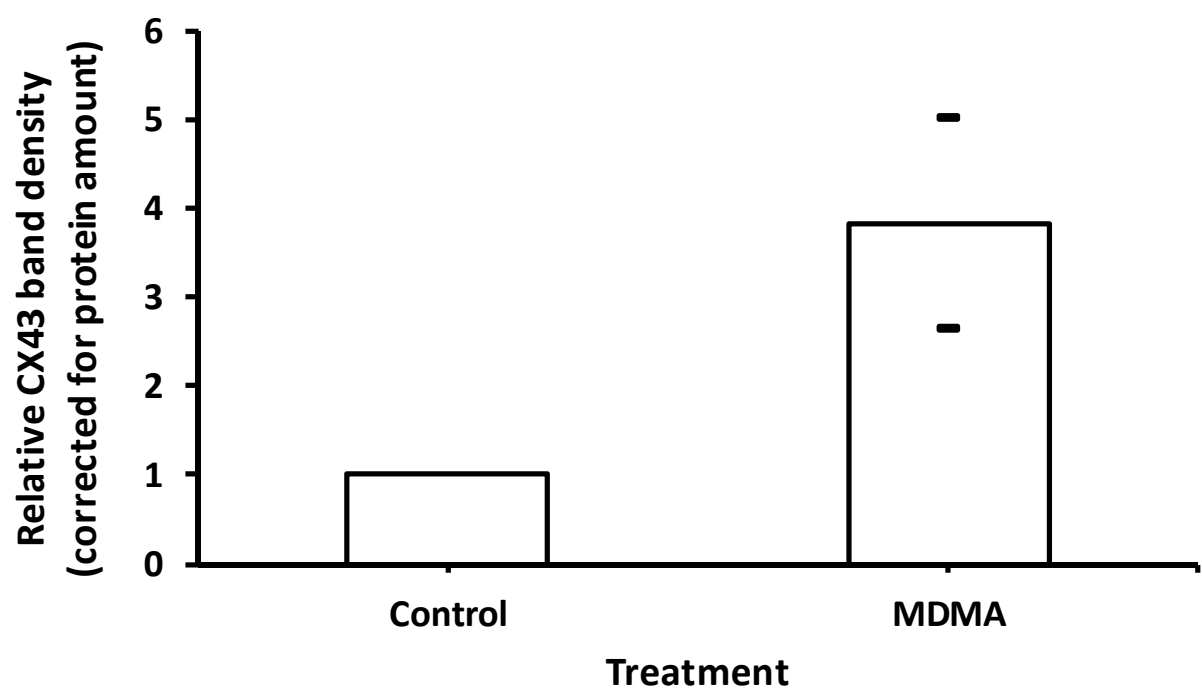

Figure 10-4: Effect of in vivo exposure to MDMA on relative CX43 levels in rat COC. The bars indicate the means, while the dashes represent each individual relative band density measurement.

The effects of administering MDMA or the vehicle alone to female rats on GDF9 protein levels in COC is depicted in Figure 10-5. Multiple bands between 45 and 90 kDa in size were observed in the positive control of both replicates. These included a band at 49 kDa which corresponds to promature GDF9. No bands were observed in the negative control of either replicate. When the GDF9-specific antibody was pre-absorbed with recombinant GDF9, no bands were detected (Figure 10-5C). Multiple bands between 45 and $70 \mathrm{kDa}$ were detected in the lanes containing COC lysate.

In the first replicate (Figure 10-5A), levels of promature GDF9 were similar in COC of MDMAtreated and control rats. Conversely in the second replicate (Figure 10-5B), levels of promature GDF9 were 4.8-fold higher in COC of MDMA-treated rats compared to that in control rats. Mature GDF9 (20 kDa) was not detected in either replicate. Due to the variable response to MDMA between replicate experiments, the effect of MDMA on GDF9 promature levels in female rats observed were equivocal (Figure 10-6). Despite this, it is evident that GDF9 promature levels do not decline in COC following in vivo exposure to MDMA in female rats. 
A

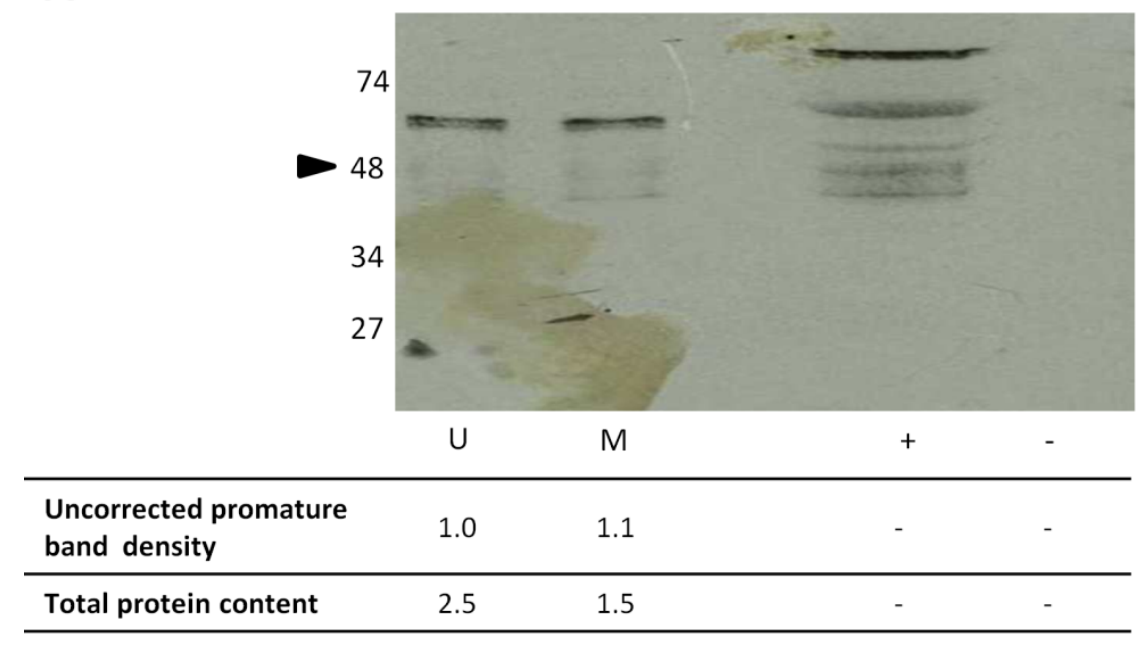

B

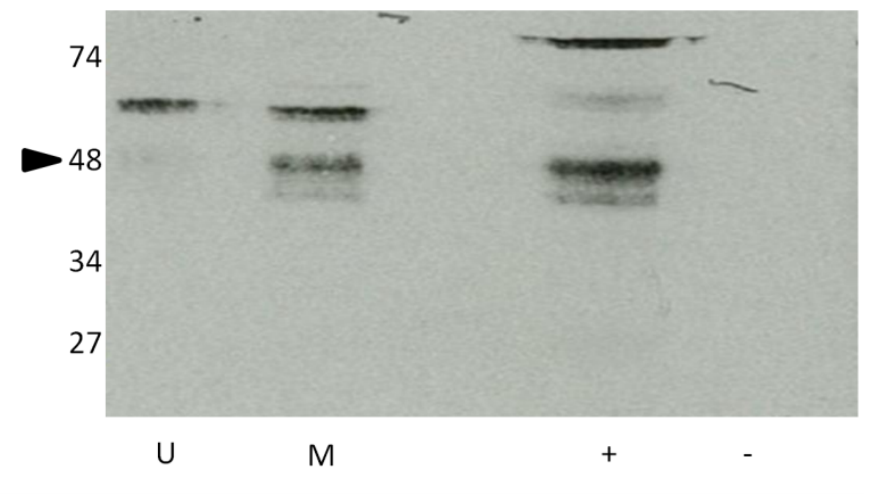

\begin{tabular}{lccll}
\hline $\begin{array}{l}\text { Uncorrected promature } \\
\text { band density }\end{array}$ & 1.0 & 4.8 & - & - \\
\hline Total protein content & 5.4 & 4.3 & - & - \\
\hline
\end{tabular}

C

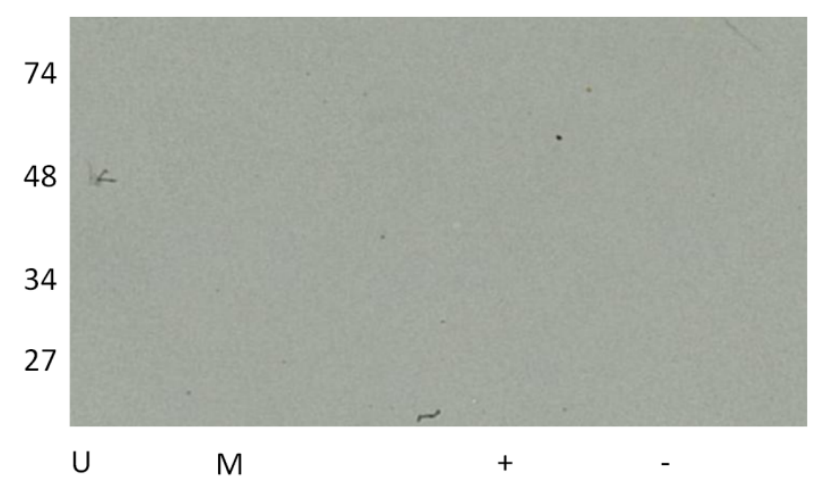

Figure 10-5: Effect of MDMA on GDF9 protein levels in COC from female rats. Western blots were performed in duplicate (A and B) to determine the level of GDF9 protein in COC lysate. COC were collected from rats injected with either the vehicle control (U), or $5 \mathrm{mg} / \mathrm{kg} /$ day MDMA (M). Rat ovary lysate was included as a positive control (+) and E. coli produced recombinant ovine BMP15 was included as a negative control (-). A control experiment $(C)$ was also performed where the blot shown in (B) was stripped and re-probed with the GDF9-specific antibody that had been pre-absorbed with E. coli produced recombinant ovine GDF9. Molecular sizes $(\mathrm{kDa})$ are indicated to the left of each blot. The density of the band of interest (indicated by an arrowhead) was measured and corrected for the control at 1 hour. Total protein $(\mu \mathrm{g})$ in each sample was determined using the BCA assay. 


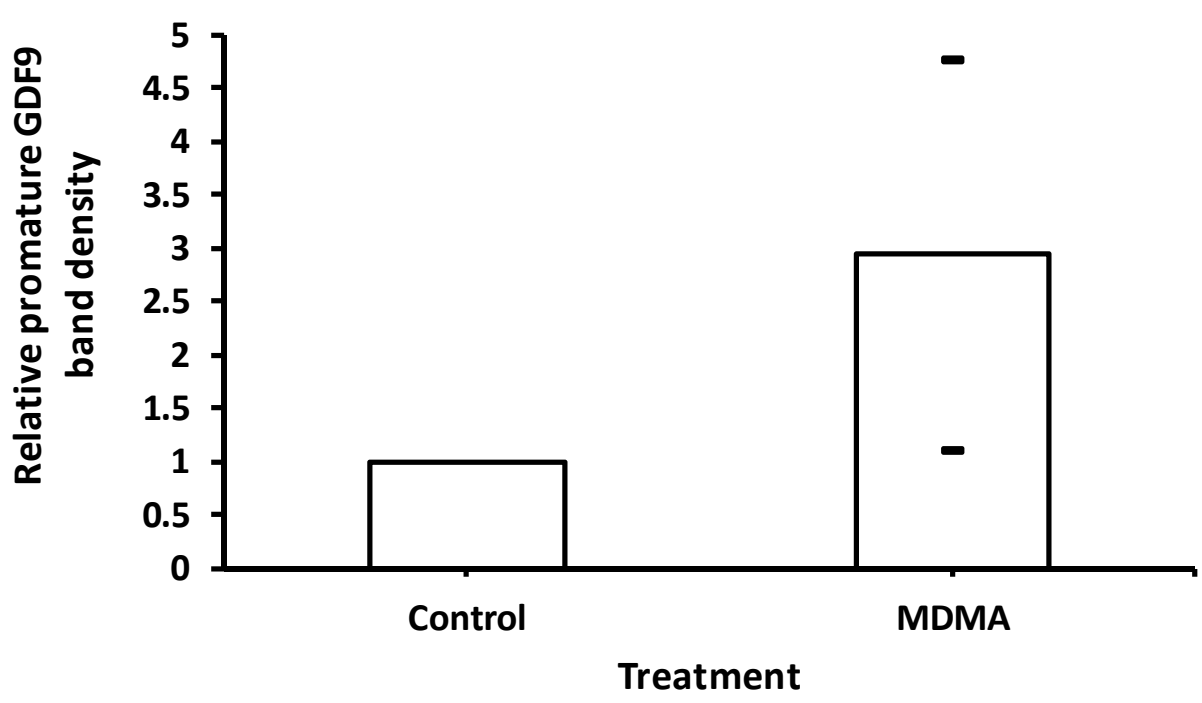

Figure 10-6: Effect of in vivo exposure to MDMA on relative promature GDF9 levels in rat COC. The bars indicate the means, while the dashes represent each individual relative band density measurement.

\subsection{Discussion}

This study indicates there is a marked increase in CX43 protein levels in COC of female rats following the administration of MDMA over three consecutive days. This observation is consistent with that observed in the in vitro study (Chapter 9) where a 25 hour incubation of rat COC with MDMA resulted in a 1.7-fold increase in CX43 levels, compared to control COC. However in contrast to the effects of MDMA in vitro, administration of MDMA to female rats did not affect oocyte meiotic arrest, candidate gene expression in CC or oocytes, or promature GDF9 protein levels in COC.

Behavioural tests were undertaken to ensure a physiologically effective dose of MDMA was administered. Rats that received a dose of $5 \mathrm{mg} / \mathrm{kg}$ MDMA were markedly more active compared to rats that received saline. Locomotor activity, as measured by ambulatory counts, is well-known to increase after exposure to MDMA and the effects are dose dependent (Callaway et al., 1990; Brennan and Schenk, 2006). The results in this present study demonstrate that the dose of MDMA administered to rats in this study was consistent with a behaviourally effective dose.

CX43 protein levels were markedly upregulated in COC from MDMA-treated rats, compared to COC from control rats. The differentially phosphorylated forms of CX43 detected in the second, but not the first, replicate, suggest that MDMA did not affect the phosphorylation of 
CX43. To the best of my knowledge, there is only one published study investigating the effect of MDMA on CX43 levels. Male rats were administered $20 \mathrm{mg} / \mathrm{kg}$ MDMA via ip injection and sacrificed at 24 hours post-MDMA administration (Zhuo et al., 2013). The levels of CX43 as detected using immunohistochemistry were markedly reduced in heart tissue of MDMA-treated, compared to control, rats.

The results presented herein suggest that MDMA increased the number of GJ channels present in the CC and that this is independent of transcription. The increase in CX43 protein may occur via several post-transcriptional or post-translational mechanisms including increased translation of mRNA stores, alternative splicing of the $C_{x} 43$ mRNA to variants with increased translation efficiency (Pfeifer et al., 2004), or inhibition of CX43 degradation. As discussed previously (Chapter 6), the implications of increased GJ communication on COC health are largely unknown. Increased nutrient transfer to the oocyte may improve oocyte quality due to the increased availability of substrates for maturation and development. However excessive nutrient availability to the oocyte can also have negative consequences. For example, excessive transfer of glucose and glucose metabolites to the oocyte results in increased utilization of alternate metabolic pathways such as the hexosamine biosynthetic, pentose phosphate or polyol pathways (Sutton-McDowall et al., 2010). While these pathways all have a role in normal oocyte maturation, abnormal upregulation can result in deleterious effects such as aberrant $O$-linked glycosylation, precocious nuclear maturation and altered intracellular redox state (Downs et al., 1998; Brownlee, 2001). In addition, previous studies have found that under hyperglycaemic conditions, increased reactive oxygen species and reduced glutathione concentrations are detected in bovine oocytes (Hashimoto et al., 2000).

For reasons unknown, the GDF9 blots were far less sensitive than those performed for the in vitro studies presented in Chapter 9. It is possible that this prevented accurate determination of the levels of promature GDF9 in these samples. Indeed, levels of GDF9 in COC from MDMA-treated rats were very variable compared to COC from the control rats. However, whilst the results presented here do not allow a definitive conclusion on the effect of in vivo exposure to MDMA on GDF9 protein levels, it is unlikely that in vivo MDMA exposure results in decreased GDF9 promature levels. This observation is supported by the 
in vitro studies herein (Chapter 9) where in vitro exposure of rat $\mathrm{COC}$ to MDMA for 25 hours resulted in increased GDF9 promature protein compared to controls.

The levels of gene expression in $C C$ and DO from rats that received MDMA were not different between treated and control rats for any of the genes tested herein. Furthermore, the proportion of oocytes that were in meiotic arrest (i.e. had a visible GV) was not different between the treated and control rats. These results contradict those observed following in vitro exposure of rat COC to MDMA (Chapter 7 and 8 ) where GVBD was promoted and levels of $C x 43, G f p t 1, C y c s$, and Pfkp mRNA in rat CC were affected. However, differences may exist in the susceptibility of the COC to MDMA between in vitro and in vivo systems. For example, the location of the $\mathrm{COC}$ in the follicular compartment where it is surrounded by both follicular fluid and GC may offer the COC greater protection from MDMA compared to isolated COC in culture. Additionally, MDMA is processed in the body into metabolites, such as MDA or HHMA that may not affect the parameters tested in this study. Another possibility is that any MDMA-mediated effects were attenuated by the time the rats were sacrificed approximately 18 hours post-injection. Studies have shown that a dose of $5 \mathrm{mg} / \mathrm{kg}$ MDMA delivered to adult female rats subcutaneously results in maximum plasma concentrations of MDMA $(293 \pm 50 \mathrm{ng} / \mathrm{mL})$ after 2 hours, while MDA reached maximum concentrations $(46 \pm 7$ $\mathrm{ng} / \mathrm{mL}$ ) after 3 hours (Fonsart et al., 2009). The half-life of both MDMA and MDA in adult female rats when a dose of $1 \mathrm{mg} / \mathrm{kg}$ was administered intravenously was 3.1 hours. This result suggests that plasma concentrations of both MDMA and MDA would be very low at 18 hours after administration. However, effects on CX43 protein levels were detected 18 hours after MDMA administration in the current study, and 24 hours after MDMA administration in a previous study (Zhuo et al., 2013).

The dose of $5 \mathrm{mg} / \mathrm{kg} /$ day used in this current study is approximately equivalent to that received in one ecstasy tablet (98 mg or $1.4 \mathrm{mg} / \mathrm{kg}$ MDMA in humans; McCann and Ricaurte, 2001). Ecstasy is typically consumed orally in humans, however in this study MDMA was administered via ip injection. A comparison between oral, ip, and subcutaneous routes of administration in rats demonstrated that a peak in plasma levels of MDMA occurred between 1-3 hours post administration, and these administration routes were comparable (Green et al., 2009). Differences in MDMA metabolism between rats and humans may complicate comparisons made between studies. For example in humans, MDMA auto- 
inhibits its own metabolism resulting in disproportionately large increases in plasma MDMA concentrations following small increases in the dose of MDMA administered (de la Torre et al., 2000). However, auto-inhibition of MDMA metabolism is not observed in rats (Green et al., 2009). In addition, the major MDMA metabolite produced in humans is 3,4dihydroxymethamphetamine (HHMA) while MDA is a minor metabolite. Conversely in rats, MDA is the major metabolite produced (Easton and Marsden, 2006) and whilst HHMA is detectable in rat urine, it is quickly methylated to 4-hydroxy-3-methoxymethamphetamine (HMMA) in blood (Fonsart et al., 2009). Moreover, different enzymes are involved in the metabolism of MDMA in humans and rats. For example the enzyme involved in the demethylation of MDMA to produce HHMA is CYP2D6 in humans and CYP2D1 in rats (Easton and Marsden, 2006). Therefore, while the results presented in this study demonstrate that in vivo exposure to MDMA results in increased $\mathrm{CX} 43$ levels in rat $\mathrm{COC}$, this may not translate in humans.

In summary, these results establish that administering $5 \mathrm{mg} / \mathrm{kg} /$ day MDMA to adolescent female rats causes a marked increase in the level of CX43 protein in COC, consistent with that of in vitro studies. Whilst the response of GDF9 protein levels to MDMA exposure was variable, it is likely not to cause a reduction GDF9 protein levels. Interestingly, despite a definitive increase in CX43 protein levels in COC after in vivo MDMA exposure, the proportion of oocytes in meiotic arrest, gene expression levels of CC-derived Atr, CX43, Cycs, Gfpt1 and Pfkp mRNA and oocyte-derived Atr, Bmp15, Cx37 and Gdf9 mRNA were unchanged. 


\section{DISCUSSION}

One of the major factors for reproductive success is the production of a healthy oocyte by the female for fertilisation. Oocyte maturation is tightly coordinated with follicular cell processes such as proliferation, metabolism, and steroidogenesis. This coordination is achieved, in part, through oocyte-secreted growth factors which modulate the aforementioned CC functions. Moreover, transfer of nutrients and small molecules from the $\mathrm{CC}$ to the oocyte via GJs facilitates cytoplasmic maturation while maintaining meiotic arrest. These oocyte growth factor and GJ communication pathways constitute a communication loop which is essential for follicular development. The major objective of this thesis was to investigate the effects of lifestyle factors on pathways involved in the bi-directional communication system of the COC.

\subsection{Method optimisation and validation}

A key component of this research was to establish, optimise and validate technologies that would enable the testing of environmental contaminants and lifestyle factors on pathways involved in the maintenance of $\mathrm{COC}$ communication. Thus, preliminary experiments were performed to: 1) establish a standard protocol to measure GJ communication in rat $\mathrm{COC}$ in vitro; 2) validate the use of calcein-treated CC and DO for multiplex qPCR; and 3) determine the health of rat COC over 25 hours in culture.

A GJ bioassay first described by Thomas et al. (2004) was modified and optimised to test the effects of specific compounds on the degree of GJ transfer in rat $\mathrm{COC}$ in culture. During in vitro culture of $\mathrm{COC}$, the intra-oocyte concentration of CAMP is critical for maintaining GJ communication in the COC and meiotic arrest of the oocyte (Wert and Larsen, 1989; Thomas et al., 2002). Elevated intra-oocyte cAMP levels within cultured COC may be attained by the addition of either cAMP stimulators or PDE inhibitors. An important and unexpected finding in this study was that the addition of forskolin, an adenylyl cyclase stimulator, did not influence GJ communication in rat COC. However, inhibition of CAMP breakdown by the addition of PDE inhibitors, specifically a combination of rolipram and dipyridamole, increased GJ communication in rat COC. This suggests a role for PDE7, PDE8, PDE10, and PDE11 in maintenance of the nutrient transfer system in this rodent species. A dosedependent inhibition of fluorescence transfer to the oocyte occurred when $\operatorname{COC}$ were 
treated with the GJ uncoupler carbenoxolone. Moreover, oocyte fluorescence was markedly inhibited when COC were treated with carbenoxolone following up to 22 hours in culture. This provides strong evidence that incorporation of calcein in the oocyte occurs predominantly via GJ transfer from the CC. Therefore, the GJ bioassay described herein is a highly specific and robust indicator of GJ activity within rat COC.

The desire to directly compare GJ activity with candidate gene expression led to the validation of a TaqMan multiplex qPCR methodology using cDNA from calcein-treated COC. Baseline fluorescence was not detected in calcein-treated samples by the Corbett RotorGene 6000 machine, and calcein treatment did not affect global gene expression levels, as measured by Rp/19 mRNA. However, calcein treatment did affect expression levels of some individual genes. Specifically, oocyte-derived Gdf9 and Cx37, and CC-derived Cycs, Gfpt1, and Atr expression levels were all influenced by calcein treatment. Notably, interaction effects were measured for the aforementioned CC genes, indicating an effect of calcein on gene expression over time. It appeared that gene expression levels in those DO and CC that had been incubated for 5 or 13 hours were more sensitive to calcein-mediated changes as excluding these samples from the analysis removed most of the treatment or interaction effects that were measured. However, a treatment effect remained for oocyte-derived Atr, and treatment and interaction effects remained for CC-derived Gfpt1.

To the best of my knowledge, this study is the first to measure GJ communication and gene expression levels in the same COC. These results show that the effect of calcein should be tested for each candidate gene, as calcein exposure may up or downregulate levels of some genes. Measurement of both GJ communication and gene expression was particularly desirable in this study as it allowed the effects of lifestyle and environmental factors on these pathways to be determined in individual COC. This is important as follicular development is hierarchical resulting in a population of follicles that are functionally distinct despite being morphologically identical. Furthermore, the correlation between GJ communication and gene expression levels may have potentially large implications for determining the genes and pathways that interact with or are affected by GJ activity, although no such correlation was observed in this study. In all experiments where the effects of lifestyle and environmental factors were being tested, both the control and treated COC were exposed to calcein. Therefore, any changes in gene expression levels between the 
treated and control groups were a result of the treatment and not a biological effect of calcein exposure. However, the effects of lifestyle and environmental factors on gene expression in $\operatorname{COC}$ that were not treated with calcein will need to be confirmed in future experiments.

In order to test the effects of prolonged exposure of rat $\mathrm{COC}$ to various compounds in vitro, the effects of culturing rat $\mathrm{COC}$ under control conditions, in regards to GJ activity, GVBD, and gene and protein expression levels, were characterised (Chapter 5). It was established that some of these parameters were regulated by time in culture. GJ communication was maintained over 49 hours of culture, and moreover was increased at the time points of 5, 19 and 25 hours. Concurrently, the proportion of meiotically-arrested oocytes (i.e. those with a visible GV) decreased over time. These results demonstrate that the reduction in the proportion of meiotically-arrested oocytes, which reached $44 \%$ by 25 hours, was not correlated to a decline in GJ communication. Compared to that after 1 hour in culture, CCderived CX43 protein levels were markedly reduced at 5 hours, but were increased at 13 and 25 hours. Interestingly, most of the oocyte genes were stable over time, with the exception of Atr mRNA levels, which were reduced after 25 hours in culture. Conversely, expression levels of all the CC-derived genes changed over time. Compared to that after 1 hour in culture, the expression levels of CC-derived Atr and Gfpt1 mRNA were higher at 13 hours, while Cycs mRNA was reduced at 25 hours. Expression levels of both $C x 43$ and Pfkp were markedly reduced from 5 hours onwards. However, given that GJ communication was maintained over time as was expression of Cycs, which is involved further down the energy production pathway, the findings here reveal that the health of the $\mathrm{COC}$ is not necessarily compromised following 25 hours in culture.

Unfortunately, mRNA expression levels of oocyte-derived Cycs and oocyte-derived and CCderived Glut1 were below detection limits. Therefore, these were unable to be included in the study.

\subsection{In vitro effects of lifestyle factors}

A summary of the in vitro effects of lifestyle factors on COC health is presented in Table 11-1. Each of those factors for which a treatment effect on GJ communication was measured (i.e. BPA, caffeine, and nicotine) had the greatest effect after 25 hours exposure. Therefore only 
the 1 and 25 hour samples were tested for gene expression levels. This also allowed exclusion of the 5 and 13 hour time points in which gene expression was found to be more sensitive to calcein-AM perturbation.

Table 11-1: Summary of the in vitro effects of lifestyle factors on COC function. Arrows indicate that the parameter was up- or down-regulated by treatment. For GJ communication and gene expression results, overall treatment effects $(P<0.05)$ are indicated by arrows alone, while a treatment $x$ time effect $(P<0.05)$ is indicated by an arrow accompanied by the time point at which an effect was noted. For protein expression, arrows indicate a trend of up- or down-regulation at a specific time point ( $>1.2$-fold change). Dashes indicate that no effect of treatment was observed for that parameter.

\begin{tabular}{|c|c|c|c|c|c|c|}
\hline & BPA & Caffeine & Ethanol & Nicotine & MDMA & THC \\
\hline $\begin{array}{c}\text { GJ } \\
\text { communication }\end{array}$ & $\uparrow$ & - & - & $\uparrow 25$ hours & - & - \\
\hline Cx43 mRNA & $\uparrow 25$ hours & $\downarrow$ & $\uparrow 1$ hour & - & $\uparrow$ & $\uparrow$ \\
\hline CX43 protein & - & $\uparrow 1$ hour & - & $\begin{array}{l}\downarrow 1 \text { hour } \\
\uparrow 25 \text { hours }\end{array}$ & $\uparrow 25$ hours & - \\
\hline Cx37 mRNA & - & - & - & $\uparrow 1$ hour & - & - \\
\hline GVBD & - & - & $\downarrow$ & - & $\uparrow$ & $\uparrow$ \\
\hline GDF9 protein & $\begin{array}{l}\downarrow 1 \text { hour } \\
\uparrow 25 \text { hours }\end{array}$ & $\begin{array}{l}\downarrow 1 \text { hour } \\
\uparrow 25 \text { hours }\end{array}$ & - & - & $\uparrow 25$ hours & $\uparrow 25$ hours \\
\hline Gfpt1 mRNA & - & - & - & - & $\uparrow$ & $\uparrow$ \\
\hline Cycs mRNA & - & - & - & - & $\downarrow 25$ hours & - \\
\hline Pfkp mRNA & - & - & - & - & $\begin{array}{l}\uparrow 1 \text { hour } \\
\downarrow 25 \text { hours }\end{array}$ & - \\
\hline
\end{tabular}


A notable finding of this study was that in general, CC-derived genes, compared to the oocyte-derived genes, were far more sensitive to all the treatments tested herein. The CC are known to protect the oocyte from reactive oxygen species (Tatemoto et al., 2000). The results from this current study support the evidence that CC have a protective role and extend this role to include protection from potentially harmful exogenous factors. Furthermore, expression of the CC-derived gene $C_{x} 43$ was significantly more sensitive to exogenous factors than any other gene tested. Indeed, $C x 43$ levels were altered by all of the treatments included in this study, although the effect of nicotine was negligible.

The concentrations of lifestyle factors chosen for this study were based upon measurements in biological fluids. Where available, the concentration of the factor in follicular fluid was used as a reference point (i.e. BPA; Ikezuki et al., 2002). In cases where this information was not available, the serum concentration was used as a reference point. In general, the concentrations used in this study were 10-fold greater than that measured in follicular fluid (i.e. BPA) or blood (i.e. caffeine, ethanol, nicotine, MDMA, and THC). The exception to this is caffeine, for which the concentration was 10-fold greater than that measured in serum (Axelrod and Reichenthal, 1953), but was 150-fold greater than that measured in follicular fluid (Al-Saleh et al., 2010). The reason for this is that the concentration of caffeine was selected prior to this information being published. Selection of high physiological concentrations of lifestyle and environmental factors for the in vitro study reduced the probability of false negative results However, due to not selecting concentrations well above the physiological range, there was increased difficulty in distinguishing real effects from the background variation. Despite this, these results provide convincing evidence that rat $\mathrm{COC}$ are sensitive to high physiological concentrations of lifestyle and environmental factors.

A major limitation of this in vitro study was that this model only explores the effects of direct exposure of the COC to lifestyle and environmental factors, and does not take into account other mechanisms by which oocyte quality is regulated (such as the hypothalamic-pituitary axis) or the potential protective effects of the follicular environment. Therefore, follow up investigations into the effects of exposure to lifestyle and environmental factors via an in vivo route (such as the in vivo MDMA experiments presented in this thesis) are necessary. 


\subsubsection{GJ communication is promoted by BPA, caffeine, and nicotine}

GJ communication and CX43 protein levels decreased over time in culture in the control COC. For reasons unknown, this was in contrast to the results in Chapter 5 where oocyte fluorescence was higher at 25 hours in culture, compared to 1 hour. Nonetheless, exposure to BPA and nicotine prevented the reduction in GJ communication over time, with higher fluorescence levels in the treated oocytes than the control oocytes at 25 hours. A similar effect was observed after exposure to caffeine, however this was just under the level of significance $(P=0.052)$. Interestingly, GJ communication was not correlated with mRNA levels of either $C_{x} 43$ or $C_{x 3} 7$ in individual samples. Furthermore, the pattern of GJ communication was not necessarily correlated with changes in CX43 protein levels in the treated COC. This is consistent with findings from Wang et al. (2009) where only very weak correlations between CX43 protein expression and GJ conductance were observed.

Post-translational modifications and incorporation into GJ plaques are essential for GJ function. In addition, only 2-20 \% of CX43 GJs are active within a GJ plaque (Bukauskas et al., 2000). Therefore, it is perhaps unsurprising that GJ communication does not necessarily correlate with mRNA and protein levels of CX43 in this study. Furthermore, it is possible that the presence of oocyte-derived GJs composed of CX37, rather than CC-derived GJs composed of $\mathrm{CX} 43$, are the rate-limiting factor for transfer of nutrients into the oocyte. An effort to measure CX37 protein levels was undertaken for this thesis however expression levels were so low that it was not feasible to include it in this study.

Interestingly, Cx43 mRNA levels were increased together with GJ communication after a 25 hour incubation with BPA. BPA has oestrogenic activity, allowing it to bind to and activate the oestrogen receptor albeit with a lower potency than endogenous oestrogens (vom Saal et al., 2007). Both $C_{x} 43$ mRNA and protein levels have been reported to be upregulated in response to oestrogens (Wiesen and Midgley, 1994), therefore these results are consistent with the oestrogenic properties of BPA. These results suggest that BPA promoted GJ communication independently of the number of CC-derived GJs present, because CX43 protein levels were unaffected. This conclusion is similar to that of a previous study that found that BPA inhibited GJ communication in an epithelial cell line independently of mRNA or protein levels of CX43 (Lee and Rhee, 2007). Further investigation into the phosphorylation states and cellular localisation of CX43 or levels of CX37 protein would 
provide further information about how BPA prevents the time-dependent decrease in GJ communication. Moreover, determining the level of CX43 protein and GJ communication at later times would reveal whether given time, the increased $C_{x} 43$ mRNA seen at 25 hours were translated into CX43 protein and formed functional GJ channels.

Initial exposure to nicotine reduced $C X 43$ protein levels and increased $C \times 37$ mRNA levels, however GJ communication was unaffected. Furthermore, a slight reduction in $C_{x} 43$ levels was observed in the nicotine-treated $\mathrm{COC}$ however this did not reach significance. After 25 hours of exposure to nicotine, GJ communication and CX43 protein levels had increased, while $C \times 37$ mRNA levels were similar to the control. These results suggest that nicotine may prevent the time-dependent decrease in CX43 protein levels, thereby maintaining GJ communication over 25 hours in culture. Given the initial increase in $C \times 37$ mRNA, the levels of CX37 protein may also have been increased at 25 hours, however this remains to be determined. The finding that CX43 protein levels were differently affected by short and long term incubations with nicotine suggests that this effect may be mediated by a metabolite. Indeed, cotinine, a stable metabolite of nicotine, has been detected in the follicular fluid of smokers (Zenzes and Reed, 1998). However, whether cotinine alters GJ communication of CX43 levels is unknown.

Caffeine initially reduced $C X 43$ mRNA and CX43 protein levels, however had no effect on GJ communication. The effect of caffeine on CX43 protein levels was attenuated by 25 hours whereas CX43 mRNA levels remained low and GJ communication was slightly increased $(P=0.052)$. Therefore, despite the increase in $G J$ communication at 25 hours in caffeinetreated $\mathrm{COC}, \mathrm{CX} 43$ protein levels were similar to the control. As with BPA, further investigation into phosphorylation states and cellular localisation of CX43 and levels of CX37 protein may provide insight into how caffeine regulates GJ communication. Indeed, caffeine is a PDE inhibitor and therefore may promote GJ communication through increased cAMP levels in the COC and promotion of PKA-mediated phosphorylation of CX43 (Mehta et al., 1992).

The implication of enhanced GJ communication on COC health is currently unclear. However increased availability of energy sources and other nutrients is likely to improve oocyte health. This is supported by the finding that increased GJ coupling in human CC cells is 
correlated to higher pregnancy rates during IVF (Wang et al., 2009). Moreover, maintenance of GJ communication during IVM of bovine COC is known to promote blastocyst formation (Atef et al., 2005). Additionally, molecules that maintain meiotic arrest in the oocyte such as CAMP and CGMP are well documented to be passed through GJs to the oocyte. Human oocytes that were pre-incubated with a PDE3 inhibitor (i.e. maintained in meiotic arrest) during IVM had higher maturation rates after removal of the inhibitor, compared to controls where the inhibitor was absent (Nogueira et al., 2006). No effect of BPA, caffeine, or nicotine on GVBD was noted in this study. However the addition of PDE inhibitors may have reduced the need for GJ transfer of molecules that maintain meiotic arrest.

Conversely, increased GJ communication may be indicative of increased energy requirements to allow the oocyte to carry out RNA surveillance and DNA damage repair in response to environmental stress (Baumann et al., 2007). The findings from this study strongly indicate that BPA and nicotine, and to a lesser extent caffeine, promote the transfer of small molecules, which may include energy substrates, to the oocyte via GJs. The metabolism of glucose via glycolysis occurs in the $\mathrm{CC}$, and subsequently lactate and pyruvate are transferred to the oocyte and undergo metabolism by the TCA cycle and oxidative phosphorylation to produce ATP. Furthermore, by-products of oxidative phosphorylation include reactive oxygen species (Voet and Voet, 2004). Increased glucose concentrations and oxidative phosphorylation are associated with the production of reactive oxygen species in oocytes (Hashimoto et al., 2000), which may result in a reduction in oocyte quality (Agarwal et al., 2003). Moreover, increased transfer of glucose metabolites to the oocyte may result in utilisation of alternate metabolic pathways such as the PPP. Hyperglycaemic conditions result in the activation of the PPP and subsequent precocious oocyte meiotic maturation (Downs et al., 1998). Therefore, increased transfer of PPP substrates to the oocyte may also result in precocious nuclear maturation, however this was not observed in this study. Further investigation into how upregulation of GJ communication affects the precise coordination of oocyte cytoplasmic and nuclear maturation is necessary. Determining whether BPA, caffeine, and nicotine promote the formation of reactive oxygen species and the activity of the PPP would reveal whether these pathways are activated by increased GJ transfer. 
Although incubation of $\mathrm{COC}$ with ethanol, MDMA, or THC did not affect GJ communication, each of these treatments did affect CX43 mRNA levels and/or CX43 protein expression. Exposure of $\mathrm{COC}$ to ethanol initially raised mRNA levels of $C_{x} 43$, but this effect was attenuated after 25 hours of exposure. MDMA increased expression levels of Cx43 mRNA regardless of exposure time, while CX43 protein levels were also increased after a 25 hour exposure. THC increased $C_{x} 43$ mRNA levels after 25 hours. These results demonstrate that mRNA and protein levels of CX43 are not good indicators of GJ communication in rat COC.

\subsubsection{GDF9 protein levels are altered by BPA, caffeine, MDMA and THC}

Exposure to BPA or caffeine for 1 hour caused a reduction in promature GDF9 levels. However, a 25 hour exposure to BPA or caffeine restored promature GDF9 levels to over and above that in the control. Moreover, exposure to MDMA or THC for 25 hours promoted promature GDF9 levels. Interestingly, GDF9 protein levels were not correlated to Gdf9 mRNA levels, which were unaffected by time or treatments. Detection of the mature form of GDF9 was inconsistent in this study therefore the major conclusions were drawn from levels of promature GDF9. The biological functions of the proregion and promature GDF9 forms are currently unknown. However, mice that were immunised against the GDF9 proregion had smaller litter sizes suggesting a functional role of the proregion in female reproduction (Mclntosh et al., 2012). Expression levels of BMP15 were low in rat COC in the current study, and therefore the measurement of BMP15 over a wide range of treatments was not feasible. However, Gdf9 and Bmp15 mRNA expression levels are known to be tightly co-regulated in several species including rat (Crawford and McNatty, 2012). In the rat, Bmp15 mRNA levels were 3.65-fold lower than Gdf9 mRNA levels, indicating a reduced requirement for BMP15 in rat follicular growth. Indeed, mature BMP15 is not detectable in the rat until just prior to ovulation, consistent with the finding that Bmp15 KO mice are fertile (Yan et al., 2001; Yoshino et al., 2006). Therefore, although BMP15 protein levels were not measured in this study, they are likely to be altered proportionately with GDF9.

Reduced GDF9 levels are likely to significantly reduce oocyte health. During follicular growth, the oocyte tightly controls CC functions including proliferation, metabolism, apoptosis, luteinisation, and expansion. It is likely that a reduction in GDF9 levels decreases CC proliferation, metabolism, and expansion but increases CC apoptosis and luteinisation. For example, downregulation of GDF9 expression using RNA interference demonstrated that CC 
expansion and expression of genes involved in CC expansion (i.e. Has2 and Ptgs2) were significantly inhibited (Gui and Joyce, 2005). Furthermore, polycystic ovarian syndrome, a syndrome characterised by anovulation, polycystic ovaries, and hyperandrogenism, is associated with reduced Gdf9 expression levels (Teixeira Filho et al., 2002). A complete lack of GDF9 (i.e. in Gdf9 KO mice) causes follicular growth to arrest at the primary stage (Yan et al., 2001).

Despite an initial reduction following exposure to BPA and caffeine, GDF9 promature levels were higher in the treated COC than the control after 25 hours. This may have been due to the action of metabolites of BPA and caffeine which stimulated GDF9 protein levels. Alternatively, the oocyte may have produced more GDF9 to stimulate CC metabolism, indicating a greater requirement for CC metabolites.

Increased GDF9 protein levels are likely to have a positive effect on follicle growth and maturation. Addition of recombinant GDF9 to is known to promote preantral mouse follicle growth in vitro (Hayashi et al., 1999), and to improve oocyte developmental competence (Hussein et al., 2006). Furthermore, higher concentrations of GDF9 were correlated with higher embryo quality during IVF (Gode et al., 2011). This positive effect of GDF9 on follicular development is likely to be mediated through increased CC proliferation and metabolism, and decreased CC steroidogenesis and apoptosis (Gilchrist et al., 2008). Indeed, GDF9 stimulates CC proliferation (Gilchrist et al., 2004b; McNatty et al., 2006), cholesterol metabolism (Su et al., 2008), and amino acid uptake (Eppig et al., 2005). However, aberrant GDF9 production may stimulate these CC functions inappropriately resulting in follicular dysfunction and thus reducing $\mathrm{COC}$ health.

There did not appear to be a correlation between GJ communication, CX43 mRNA, CX43 protein levels, and GDF9 protein levels. CX43 is thought to positively regulate TGF $\beta$ signalling, through competitive interactions with SMAD2/3 on microtubules (Dai et al., 2007). Thus although protein levels of CX43 and GDF9 were not correlated, it is possible that GDF9 signalling was correlated to CX43 protein, however this was not investigated.

\subsubsection{GVBD is prevented by ethanol but promoted by MDMA and THC}

Long exposures to ethanol (i.e. $>5$ hours) resulted in reduced proportions of oocytes undergoing GVBD. These results suggest that ethanol prevents oocyte meiotic maturation 
without inhibiting the transfer of the meiosis-suppressing molecules to the oocyte as GJ communication was unaffected. Artificial maintenance of meiotic arrest allows for cytoplasmic maturation to complete, and promotes oocyte maturation rates during IVM once the inhibitor is removed (Nogueira et al., 2006). Therefore, further investigations into whether oocytes are subsequently able to undergo GVBD when ethanol is removed are needed. Heavy alcohol consumption is currently known to cause anovulation by inhibiting the LH surge. If ethanol completely prevents GVBD in some oocytes, then the results presented here suggest that ethanol may act at both the pituitary and the follicle to prevent ovulation.

MDMA and THC both promoted oocyte GVBD when COC were incubated with these treatments for $>5$ hours. Furthermore, no additional oocytes appeared to have undergone GVBD at 13 or 25 hours compared to 5 hours, in either the MDMA- or THC-treated groups. This suggests that MDMA and THC stimulated GVBD by 4 hours. Interestingly, at 4 hours MDMA and THC had promoted GVBD in $75 \%$ and $58 \%$ of COC, compared with the control where $17 \%$ had undergone GVBD. This suggests that MDMA has a stronger influence on GVBD than THC. Moreover, these results highlight that the effects of contaminants are not identical for different COC. For example, $17 \%$ of oocytes retained their GV even in the presence of MDMA for 25 hours. Whether these oocytes were meiotically incompetent, or whether they were resistant to MDMA is unknown.

THC has previously been found to promote GVBD in rat COC in vitro (Reich et al., 1982). The results from this study suggest that meiotic maturation in oocytes is more sensitive to THC than previously thought as this study used a concentration of THC that was $\sim 30$-fold lower than that in the Reich study. Precocious resumption of meiotic maturation is thought to reduce oocyte quality due to inadequate cytoplasmic maturation. In vivo, the oocyte gains meiotic competence around the time of follicular antrum formation (Edson et al., 2009) however meiotic arrest must be maintained to allow cytoplasmic maturation such as organelle and cytoskeleton reorganization and storage of mRNAs to take place (Marteil et al., 2009). Therefore, the current study suggests that MDMA and THC may reduce COC health by promoting precocious nuclear maturation. 
No correlation between the effects of treatments on GVBD and GJ communication was noted. The transfer of CAMP and CGMP from the CC to the oocyte through GJs is essential for maintenance of oocyte meiotic arrest. However, after the LH surge CX43 is phosphorylated, resulting in closure of the GJs and initiation of GVBD (Norris et al., 2008). However, it is possible that the addition of PDE inhibitors in the current study reduced the need for CCderived CAMP and CGMP for maintenance of meiotic arrest. Both BPA and nicotine are reported to cause meiotic abnormalities (Mailhes et al., 2000; Liu et al., 2007; EichenlaubRitter et al., 2008). BPA suppresses oocyte meiotic maturation in mice (Eichenlaub-Ritter et al., 2008), while exposure to nicotine promoted meiotic maturation in vivo, but not in vitro in mice (Mailhes et al., 2000). Moreover, caffeine is a PDE inhibitor that prevents meiotic resumption in a reversible manner by increasing cAMP levels within the oocyte (Jagiello et al., 1972; Kren et al., 2004). Therefore, measuring the effects of those treatments that affected GJ communication (i.e. BPA, caffeine, and nicotine) in the absence of PDE inhibitors may have demonstrated a link between GJ communication and meiotic arrest.

\subsubsection{MDMA and THC alter expression of genes involved in glucose metabolism}

Initial exposure to MDMA resulted in increased Gfpt1 and Pfkp mRNA levels. Exposure to MDMA for 25 hours resulted in increased Gfpt1, but reduced Cycs and Pfkp mRNA levels. These results suggest that metabolism of glucose via the glycolytic and hexosamine biosynthetic pathways are promoted after a 1 hour exposure to MDMA. However, after 25 hours exposure to MDMA, glycolysis and oxidative phosphorylation were reduced and hexosamine biosynthesis was increased. Oocytes that are undergoing meiosis (i.e. after GVBD and before metaphase II arrest) consume more pyruvate than GV or MII oocytes (Downs et al., 2002). Therefore, the increase in Pfkp levels at 1 hour in the MDMA-treated group may correlate with the induction of GVBD and thus an increased requirement for energy. Although expression levels of Pfkp were increased at 1 hour, GJ communication was similar to the controls suggesting that transfer of glucose metabolites to the oocyte remained stable. Perhaps by 25 hours, meiosis had progressed such that the need for energy substrates (and $P f k p$ and Cycs expression) had abated. The expression levels of $P f k p$ did not correlate to levels of GDF9 in this study. However, GDF9 has not been implicated in the regulation of $P f k p$ in the mouse, with BMP15 and FGF8 reported to be the main regulators of glycolysis (Sugiura et al., 2008). 
The promotion of Gfpt1 levels at both exposure times by MDMA and THC, suggest that glucose metabolism through the hexosamine biosynthetic pathway is upregulated. Interestingly, GDF9 protein levels were also promoted by incubation with MDMA and THC for 25 hours, correlating to the promotion of Gfpt1 and consistent with the role of GDF9 in promoting CC expansion (Elvin et al., 1999). However, CC expansion was not observed in either the MDMA or THC-treated COC. This may indicate that glucose was used to produce substrates for $O$-linked glycosylation, rather than hyaluronic acid. Treating porcine and bovine $\mathrm{COC}$ with glucosamine, which enters the hexosamine biosynthetic pathway downstream of GFPT1, inhibited embryo development, and this effect was blocked when $O$ linked glycosylation was inhibited (Schelbach et al., 2010). Therefore, determining whether MDMA and THC promote $O$-linked glycosylation or hyaluronic acid synthesis would reveal the consequence of upregulation of the hexosamine biosynthetic pathway.

\subsection{In vivo effects of MDMA}

There is currently very little research on the effects of MDMA on female fertility. Given the finding that in vitro exposure to MDMA affects several pathways involved in $\mathrm{COC}$ function, we chose to investigate the effects of in vivo exposure to MDMA on these pathways. In vivo exposure to MDMA resulted in upregulation of CX43 protein in rat COC. GJ communication in rat COC exposed to MDMA in vivo was not measured, as in vitro exposure to MDMA did not affect GJ communication (Chapter 6). However, given the finding that CX43 protein levels in $\mathrm{COC}$ were enhanced in rats administered MDMA, further investigation into whether this affects GJ activity is necessary. Interestingly, the findings from the in vitro experiments, i.e. MDMA exposure modulates $C x 43, C y c s$, Gfpt1 and Pfkp mRNA expression and GDF9 protein levels in rat $\mathrm{COC}$, were not mirrored in the in vivo experiment. Furthermore, MDMA promoted GVBD in vitro, but did not affect meiotic resumption in oocyte in vivo. These differences between the in vitro and in vivo effects of MDMA on COC health may be due to the delivery of MDMA to the ovary or metabolic processing of MDMA in the circulation. It remains to be determined whether MDMA or its major metabolites are detectable in the follicular fluid. As discussed in Chapter 10, the major metabolic pathways differ between humans and rats. Therefore, future studies may include investigation of the actions of HHMA and MDA on $\mathrm{COC}$ health. In addition, prospective studies investigating MDMA usage and infertility in human populations would provide valuable information regarding the influence 
of MDMA on female fertility. However, this study provides evidence that MDMA regulates the COC communication network in vivo.

\subsection{Future directions}

Clear differences between the effects of in vitro and in vivo exposure to MDMA on COC function were observed. These results demonstrate the need for in vivo studies to complement in vitro studies. The in vitro studies from this thesis demonstrate that all of the treatments investigated (i.e. BPA, caffeine, ethanol, nicotine, MDMA, and THC) affect some facet of oocyte function. Therefore, investigation into the effects of in vivo exposure to each of these factors would provide further information about their influences on oocyte health. Subsequent mating studies would provide further information about the effect of these lifestyle factors and environmental contaminants on fertility.

The finding that GJ communication is not correlated to CX43 protein or CX43 mRNA levels was surprising, although not unprecedented. These findings suggest that other regulatory mechanisms affecting post-translational modifications and/or formation of GJ plaques may play a critical role in regulating function GJ communication. Further investigations into the effects of BPA, caffeine, and nicotine on localisation of CX43 and the phosphorylation states may provide more insight into how these treatments affect overall GJ communication.

In addition, assays investigating oocyte-secreted growth factor actions and glucose metabolic functions would provide further information regarding the effects of treatment on the activity of these pathways. These studies have shown that Gdf9 mRNA and GDF9 protein levels were also not correlated, perhaps due to several post-translational processing steps. It is possible that the protein data were confounded by measurement mostly of intracellular content rather than the forms secreted into the media. It is noteworthy too that the bioactivity of the promature protein, the major form detected here, is unknown. Therefore, measurements of secreted protein forms and applying appropriate bioassays to assess the bioactivity of GDF9 (e.g. GC proliferation assays) would shed light on how these treatments affect oocyte growth factor function.

The lifestyle factors were tested in isolation in the current study. However, investigation into effects of, and interactions between, multiple factors is a more realistic model. For example, 
correlation between the use of tobacco, alcohol, and cannabis is thought to be around 0.3 suggesting a relatively high incidence of co-exposure (Lynskey et al., 1998).

Although these results clearly suggest a role for lifestyle factors in regulating $\mathrm{COC}$ function, this study only examined antral follicles (i.e. fully grown oocytes). GJs are expressed in the follicle from the primordial stage (Kidder and Mhawi, 2002), and are positively regulated by FSH levels (Granot and Dekel, 1997). CX43 mRNA is abolished after the LH surge, and CX43 protein levels are reduced. Furthermore, GDF9 is expressed from the primary stage onwards in rodent follicles (Laitinen et al., 1998). Follicles at different stages of growth may be more sensitive to certain contaminants. For example, radiation therapy primarily targets primordial, rather than growing follicles (Armstrong, 1986). Therefore, investigation into the effects of these lifestyle factors on all stages of follicles will be necessary.

\subsection{Conclusion}

The major objective of this study was to determine whether exposure to BPA, caffeine, ethanol, nicotine, MDMA, or THC influences the function of rat $\mathrm{COC}$, and in particular the pathways involved in bi-directional communication between the $\mathrm{CC}$ and oocyte, that may adversely affect health.

The results presented in this study implicate the bi-directional communication loop and glucose metabolic pathways as potential targets for lifestyle factors. Exposure of $\mathrm{COC}$ in vitro to each of the contaminants included in this study affected at least one of the parameters tested. Furthermore, this study has identified that Cx43 mRNA levels are particularly sensitive to contaminants however the levels of $C_{x 43}$ mRNA were not an indicator of functional GJ communication in rat COC. The CC appeared to play a protective role, preserving the oocyte from damage caused by harmful factors in the COC microenvironment. The bi-directional communication loop and glucose metabolic pathways appeared to be less sensitive to perturbation by MDMA after in vivo exposure, compared to in vitro exposure. Therefore, delivery of lifestyle factors in the blood to the ovary, metabolism of these factors, and the containment of the $\mathrm{COC}$ within the follicle may be important contributors to the effects of contaminants on COC function. 
It is concluded that GJ communication, GDF9 protein levels, expression levels of genes involved in glucose metabolism, and oocyte meiotic arrest are sensitive processes that may be influenced by contaminants within the $\mathrm{COC}$ microenvironment. 


\section{APPENDIX 1 CARBENOXOLONE ACTION TIME}

An experiment was performed to determine how long carbenoxolone takes to inhibit dye transfer in rat COC. Briefly, COC were isolated as described in Sections 2.1.1 and 2.1.2. All steps were performed in the presence of $50 \mu \mathrm{M}$ dipyridamole and $50 \mu \mathrm{M}$ rolipram. The incubations conditions used were $37{ }^{\circ} \mathrm{C}, 5 \% \mathrm{CO}_{2}$, and $96 \%$ humidity. The 0 hour control group was not exposed to carbenoxolone, and was incubated for 0 hours prior to exposure to calcein-AM and further 1 hour incubation as described below. The other groups were incubated in the absence of carbenoxolone for varying times, such that the total incubation time was equivalent to 240 minutes for all groups, prior to being incubated in $1 \mathrm{mM}$ carbenoxolone for $0,30,60,120,180$, or 240 minutes (Table A1-1). Thereafter, the COC were incubated with $2 \mu \mathrm{M}$ calcein-AM and $1 \mathrm{mM}$ carbenoxolone for 10 minutes. The COC were transferred into calcein-AM free incubation media containing $1 \mathrm{mM}$ carbenoxolone and incubated for a further hour. The oocyte was denuded and oocyte fluorescence measured as described previously (Section 2.1.3).

Table A1-1: Incubation conditions for determining time required for maximal inhibition of dye transfer by 1 $\mathrm{mM}$ carbenoxolone. Note that the total pre-calcein incubation time is identical between all groups.

\begin{tabular}{cccc}
\hline Group & $\begin{array}{c}\text { Initial incubation } \\
(\mathbf{m i n})\end{array}$ & $\begin{array}{c}\text { Carbenoxolone incubation } \\
(\mathbf{m i n})\end{array}$ & $\begin{array}{c}\text { Total pre-calcein incubation } \\
(\mathbf{m i n})\end{array}$ \\
\hline $\mathbf{0}$ & 240 & 0 & 240 \\
$\mathbf{3 0}$ & 210 & 30 & 240 \\
60 & 180 & 60 & 240 \\
120 & 120 & 120 & 240 \\
180 & 60 & 180 & 240 \\
$\mathbf{2 4 0}$ & 0 & 240 & 240 \\
\hline
\end{tabular}


Maximal inhibition occurred following a 2 hour exposure to carbenoxolone prior to incubation with calcein-AM $(P<0.0001)$ (Figure A1-1). An exposure time of 2 hours was therefore used to determine the specificity of the GJ bioassay over time (Section 3.3.7). The incubation time of 4 hours resulted in significantly increased $(P<0.05$; Mann-Whitney test) fluorescent intensity in the control oocytes (no carbenoxolone), compared to that at 0 hours (i.e. note the $\sim 1.5$ fold higher value; Figure A1-1).

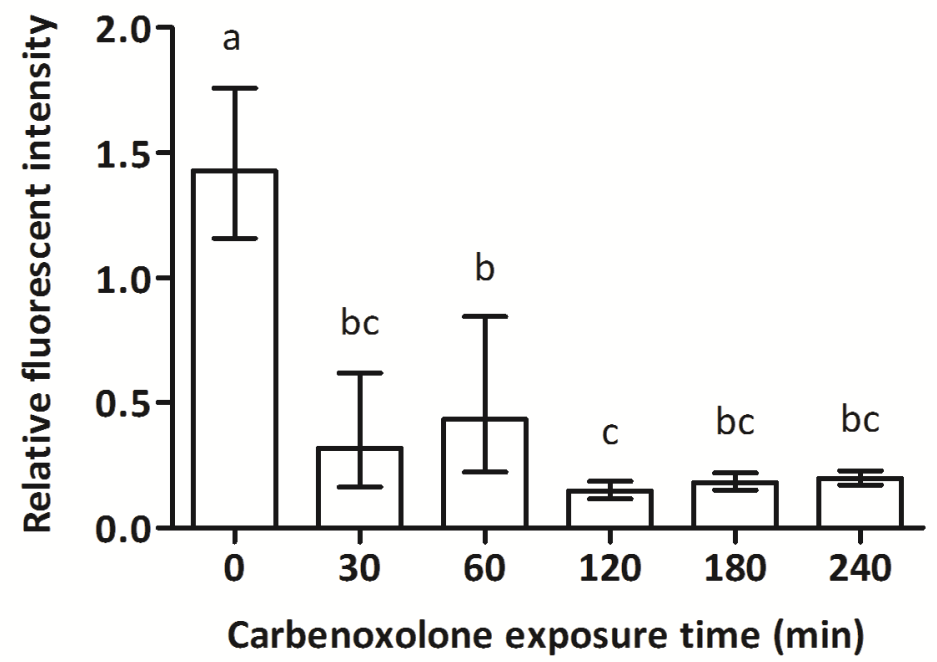

Figure A1-1: Effect of exposure time to $1 \mathrm{mM}$ carbenoxolone on fluorescent dye transfer from adjacent CC to oocytes after a 4 hour incubation of rat COC. Values are shown as geometric means $\pm 95 \%$ confidence intervals of fluorescent ratios normalised against the geometric mean of the T0 control group (not shown, geometric mean=1.0). The data were generated from 2 replicate experiments with a total of 24-32 readings from individual oocytes in each group. The data were analysed by a Kruskal-Wallis test and where significant differences were observed, a Dunn's multiple comparison post hoc test was performed. Groups that do not share a letter are significantly different $(P<0.05)$. The mean \pm SEM fluorescent reading in the T0 control group was $284 \pm 31$. 


\section{APPENDIX 2 STOCK SOLUTIONS}

\section{A2.1 GJ Assay Solutions}

\section{Dissection Media}

$0.1 \%$ Bovine serum albumin

$50 \mathrm{mg}$

$100 \mathrm{IU} / \mathrm{mL}$ Penstrep

$500 \mu \mathrm{L}$

15 mM Hepes Buffer $750 \mu \mathrm{L}$

Lebowitz media containing phenol red and L-glutamine

$43.7 \mathrm{~mL}$

Adjust $\mathrm{pH}$ to 7.4 . Store at $4{ }^{\circ} \mathrm{C}$.

\section{Incubation Media}

$0.3 \mathrm{mg} / \mathrm{mL}$ polyvinyl alcohol

$15 \mathrm{mg}$

M199 media containing L-glutamine, $2.2 \mathrm{~g} / \mathrm{L}$ sodium

bicarbonate, Earle's salt (phenol red free)

$45 \mathrm{~mL}$

Adjust $\mathrm{pH}$ to 7.4 . Store at $4{ }^{\circ} \mathrm{C}$.

\section{Wash Media}

$0.1 \%$ Bovine serum albumin

$50 \mathrm{mg}$

$100 \mathrm{IU} / \mathrm{mL}$ Penstrep

$500 \mu \mathrm{L}$

M199 media containing L-glutamine, $2.2 \mathrm{~g} / \mathrm{L}$ sodium

bicarbonate, Earle's salt (phenol red free)

$44.5 \mathrm{~mL}$

Adjust $\mathrm{pH}$ to 7.4 . Store at $4{ }^{\circ} \mathrm{C}$.

\section{1 mM calcein-AM solution}

Dissolve a $50 \mu \mathrm{g}$ aliquot in $50 \mu \mathrm{L}$ of DMSO. Store at $-20^{\circ} \mathrm{C}$. Prior to use in the GJ assay, calcein-AM was diluted 1:5 with incubation media to make a $200 \mu \mathrm{M}$ intermediate dilution. This $100 x$ calcein-AM solution was then added to incubation media, containing the appropriate treatments, at a final concentration of $2 \mu \mathrm{M}$. 
200x dipyridamole/rolipram working stock

Dilute $1 \mathrm{mg}$ dipyridamole in $99 \mu \mathrm{L}$ DMSO

Dilute $1 \mathrm{mg}$ rolipram in $182 \mu \mathrm{L}$ DMSO

Mix these solutions in a ratio of $1: 1$. Store at $-20^{\circ} \mathrm{C}$.

25x Protease Inhibitor stock

Resuspend one tablet in $2 \mathrm{~mL}$ of $\mathrm{dH}_{2} \mathrm{O}$. Aliquot into smaller volumes and store at $-20^{\circ} \mathrm{C}$

PBS

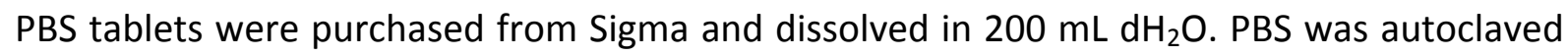
and stored at $4{ }^{\circ} \mathrm{C}$.

100x carbenoxolone stock

Carbenoxolone was purchased from Sigma, was dissolved in $\mathrm{dH}_{2} \mathrm{O}$ to a concentration of 100 $\mathrm{mM}$, and stored at $-20^{\circ} \mathrm{C}$.

\section{A2.2 SDS-PAGE stocks}

$0.5 \mathrm{M}$ Tris- $\mathrm{HCl} \mathrm{pH} 6.8$

Tris base

$6.06 \mathrm{~g}$

Make up to $100 \mathrm{~mL}$ with $\mathrm{dH}_{2} \mathrm{O}$. Adjust $\mathrm{pH}$ to 6.8. Store at room temperature.

\section{$1.5 \mathrm{M}$ Tris- $\mathrm{HCl} \mathrm{pH} 8.8$}

Tris base

Make up to $200 \mathrm{~mL}$ with $\mathrm{dH}_{2} \mathrm{O}$. Adjust $\mathrm{pH}$ to 8.8. Store at room temperature.

$10 \%(w / v)$ ammonium persulphate

Ammonium persulphate

$1 \mathrm{~g}$

Make up to $10 \mathrm{~mL}$ with $\mathrm{dH}_{2} \mathrm{O}$. Distribute into $1 \mathrm{~mL}$ aliquots. Store at $-20{ }^{\circ} \mathrm{C}$ or at $4{ }^{\circ} \mathrm{C}$ for up to 1 month. 
$10 \%$ SDS

SDS

$2 \mathrm{~g}$

Make up to $20 \mathrm{~mL}$ with $\mathrm{dH}_{2} \mathrm{O}$, store at room temperature.

$30 \%$ bis-acrylamide mix

The bis-acrylamide mix was purchased from BioRad (Auckland, NZ) and stored at $4{ }^{\circ} \mathrm{C}$.

TEMED

TEMED was purchased from BioRad and stored at room temperature.

$13.5 \%$ separating gel

$\mathrm{dH}_{2} \mathrm{O}$

$2.85 \mathrm{~mL}$

1.5 M Tris- $\mathrm{HCl}$ pH 8.8

$2.5 \mathrm{~mL}$

$10 \%$ SDS

$100 \mu \mathrm{L}$

$30 \%$ bis-acrylamide mix

$4.5 \mathrm{~mL}$

$10 \%$ ammonium persulphate

$50 \mu \mathrm{L}$

TEMED

$5 \mu \mathrm{L}$

Set up small and large glass plates in a vertical gel holder. Distribute the separating gel mix into the plates, layer a small layer of $\mathrm{dH}_{2} \mathrm{O}$ over the gel, and allow the gel to polymerise.

$4 \%$ stacking gel

$\mathrm{dH}_{2} \mathrm{O}$

$3.05 \mathrm{~mL}$

$0.5 \mathrm{M}$ Tris- $\mathrm{HCl} \mathrm{pH} 6.8$

$1.25 \mathrm{~mL}$

$10 \%$ SDS

$50 \mu \mathrm{L}$

$30 \%$ bis-acrylamide mix

$650 \mu \mathrm{L}$

$10 \%$ ammonium persulphate

$25 \mu \mathrm{L}$

TEMED

$5 \mu \mathrm{L}$

Remove the layer of water from the separating gel. Distribute the stacking gel over the top of the separating gel. Insert a 10 or 15 well comb, and allow the gel to polymerise. 
$5 x$ sample buffer

0.5 M Tris- $\mathrm{HCl}$ pH 6.8

$1.0 \mathrm{~mL}$

Glycerol

$0.8 \mathrm{~mL}$

$10 \%$ SDS

$4.6 \mathrm{~mL}$

DTT

$80 \mathrm{mg}$

$1.0 \%(\mathrm{w} / \mathrm{v})$ bromophenol blue

$0.2 \mathrm{~mL}$

$\mathrm{dH}_{2} \mathrm{O}$

$0.8 \mathrm{~mL}$

$\beta$-mercaptoethanol

$1.74 \mathrm{~mL}$

Store at $-20^{\circ} \mathrm{C}$.

5x SDS-PAGE Running Buffer

Glycine

$72 \mathrm{~g}$

Tris base

$15 \mathrm{~g}$

SDS

$5 \mathrm{~g}$

Make up to $1 \mathrm{~L}$ with $\mathrm{dH}_{2} \mathrm{O}$ and adjust $\mathrm{pH}$ to 8.3 , and store at room temperature.

\section{A2.3 Western blotting solutions}

10x Tris-glycine stock

Tris base

$30.3 \mathrm{~g}$

Glycine

$144 \mathrm{~g}$

Make up to $1 \mathrm{~L}$ with $\mathrm{dH}_{2} \mathrm{O}$, store at room temperature.

Transfer buffer

10x Tris-glycine stock

$200 \mathrm{~mL}$

Methanol

$400 \mathrm{~mL}$

Make up to $2 \mathrm{~L}$ with $\mathrm{dH}_{2} \mathrm{O}$ and store at $4^{\circ} \mathrm{C}$.

Ponceau S Stain

Ponceau S

$1.0 \mathrm{~g}$

Acetic Acid

$2.0 \mathrm{~mL}$

Make up to $200 \mathrm{~mL}$ with $\mathrm{dH}_{2} \mathrm{O}$. Store at room temperature. 
$1 \mathrm{M}$ Tris $\mathrm{pH} 7.5$

Tris base

$30.29 \mathrm{~g}$

Make up to $250 \mathrm{~mL}$ with $\mathrm{dH}_{2} \mathrm{O}$.

$\mathrm{pH}$ to 7.5

Make up to $250 \mathrm{~mL}$ with sterile water and adjust $\mathrm{pH}$ to 7.5 Store at room temperature.

TBS

$1 \mathrm{M}$ Tris- $\mathrm{HCl} \mathrm{pH} 7.5$

$20 \mathrm{~mL}$

$\mathrm{NaCl}$

$8.76 \mathrm{~g}$

Tween20

$1 \mathrm{~mL}$

Make up to $1 \mathrm{~L}$ with $\mathrm{dH}_{2} \mathrm{O}$. Store at room temperature.

$5 \%$ low-fat milk powder solution

Low-fat milk powder

$2.5 \mathrm{~g}$

TBS

$50 \mathrm{~mL}$

Make up fresh for each experiment. Store at $4{ }^{\circ} \mathrm{C}$.

\section{A2.4 ECL reagents}

1 M Tris- $\mathrm{HCl}$ pH 8.5

Tris base

$30.29 \mathrm{~g}$

Make up to $250 \mathrm{~mL}$ with $\mathrm{dH}_{2} \mathrm{O}$ and adjust $\mathrm{pH}$ to 8.5. Store at room temperature.

$90 \mathrm{mM}$ p-coumaric acid

p-coumaric acid

$0.15 \mathrm{~g}$

Make up to $10 \mathrm{~mL}$ with DMSO. Store at $-20^{\circ} \mathrm{C}$.

$250 \mathrm{mM}$ luminol

Luminol

Make up to $10 \mathrm{~mL}$ with DMSO. Store at $-20^{\circ} \mathrm{C}$. 
Solution 1

$250 \mathrm{mM}$ luminal

$100 \mu \mathrm{L}$

$90 \mathrm{mM}$ p-coumaric acid

$44 \mu \mathrm{L}$

$1 \mathrm{M}$ Tris- $\mathrm{HCl}$ pH 8.5

$1 \mathrm{~mL}$

$\mathrm{dH}_{2} \mathrm{O}$

$8.85 \mathrm{~mL}$

\section{Solution 2}

$30 \%$ hydrogen peroxide

$6 \mu \mathrm{L}$

$1 \mathrm{M}$ Tris- $\mathrm{HCl}$ pH 8.5

$1 \mathrm{~mL}$

$\mathrm{dH}_{2} \mathrm{O}$

$9 \mathrm{~mL}$

Prepare Solutions 1 and 2 fresh for each experiment. Mix together just prior to performing Western development.

\section{A2.5 Stripping solutions}

$62.5 \mathrm{mM}$ Tris $\mathrm{pH} 6.7$

Tris base $1.89 \mathrm{~g}$

Make up to $250 \mathrm{~mL}$ with $\mathrm{dH}_{2} \mathrm{O}$. Adjust $\mathrm{pH}$ to 6.7. Store at room temperature

Membrane stripping buffer

$62.5 \mathrm{mM}$ Tris pH 6.7

$50 \mathrm{~mL}$

SDS

$1 \mathrm{~g}$

$\beta$-mercaptoethanol $350 \mu \mathrm{L}$ 


\section{REFERENCES}

Aaltonen, J., Laitinen, M.P., Vuojolainen, K., Jaatinen, R., Horelli-Kuitunen, N., Seppä, L., Louhio, H., Tuuri, T., Sjöberg, J., Bützow, R., Hovata, O., Dale, L., Ritvos, O., 1999. Human growth differentiation factor 9 (GDF-9) and its novel homolog GDF-9B are expressed in oocytes during early folliculogenesis. J. Clin. Endocrinol. Metab. 84, 2744-2750.

Abraham, R.T., 2001. Cell cycle checkpoint signaling through the ATM and ATR kinases. Genes Dev. 15, 2177-2196.

Ackert, C.L., Gittens, J.E.I., O’Brien, M.J., Eppig, J.J., Kidder, G.M., 2001. Intercellular communication via connexin43 gap junctions is required for ovarian folliculogenesis in the mouse. Dev. Biol. 233, 258-270.

Aerts, J., Bols, P., 2010. Ovarian Follicular Dynamics: A Review with Emphasis on the Bovine Species. Part I: Folliculogenesis and Pre-antral Follicle Development. Reprod. Domest. Anim. 45, 171-179.

Agarwal, A., Saleh, R.A., Bedaiwy, M.A., 2003. Role of reactive oxygen species in the pathophysiology of human reproduction. Fertil. Steril. 79, 829-843.

Ahmad, S., Evans, W.H., 2002. Post-translational integration and oligomerization of connexin 26 in plasma membranes and evidence of formation of membrane pores: implications for the assembly of gap junctions. Biochem. J. 365, 693-699.

Al-Saleh, I., El-Doush, I., Grisellhi, B., Coskun, S., 2010. The effect of caffeine consumption on the success rate of pregnancy as well various performance parameters of in-vitro fertilization treatment. Med. Sci. Monit. Int. Med. J. Exp. Clin. Res. 16, CR598-605.

Aldridge, G.M., Podrebarac, D.M., Greenough, W.T., Weiler, I.J., 2008. The use of total protein stains as loading controls: an alternative to high-abundance single-protein controls in semi-quantitative immunoblotting. J. Neurosci. Methods 172, 250-254.

Amsterdam, A., Koch, Y., Lieberman, M., Lindner, H., 1975. Distribution of binding sites for human chorionic gonadotropin in the preovulatory follicle of the rat. J. Cell Biol. 67, 894-900.

Anderson, K., 2010. Lifestyle factors in people seeking infertility treatment-A review. Aust. N. Z. J. Obstet. Gynaecol. 50, 8-20.

Arellano, R.O., Martínez-Torres, A., Garay, E., 2002. Ionic currents activated via purinergic receptors in the cumulus cell-enclosed mouse oocyte. Biol. Reprod. 67, 837-846. 
Armstrong, D.T., 1986. Environmental stress and ovarian function. Biol. Reprod. 34, 29-39.

Atef, A., François, P., Christian, V., Marc-André, S., 2005. The potential role of gap junction communication between cumulus cells and bovine oocytes during in vitro maturation. Mol. Reprod. Dev. 71, 358-367.

Augood, C., Duckitt, K., Templeton, A.A., 1998. Smoking and female infertility: a systematic review and meta-analysis. Hum. Reprod. Oxf. Engl. 13, 1532-1539.

Augustin, R., Pocar, P., Navarrete-Santos, A., Wrenzycki, C., Gandolfi, F., Niemann, H., Fischer, B., 2001. Glucose transporter expression is developmentally regulated in in vitro derived bovine preimplantation embryos. Mol. Reprod. Dev. 60, 370-376.

Axelrod, J., Reichenthal, J., 1953. The fate of caffeine in man and a method for its estimation in biological material. J. Pharmacol. Exp. Ther. 107, 519-523.

Barenys, M., Gomez-Catalan, J., Camps, L., Teixido, E., de Lapuente, J., Gonzalez-Linares, J., Serret, J., Borras, M., Rodamilans, M., Llobet, J.M., 2010. MDMA (ecstasy) delays pubertal development and alters sperm quality after developmental exposure in the rat. Toxicol. Lett. 197, 135-142.

Barenys, M., Macia, N., Camps, L., de Lapuente, J., Gomez-Catalan, J., Gonzalez-Linares, J., Borra, M., Rodamilans, M., Llobet, J., 2009. Chronic exposure to MDMA (ecstasy) increases DNA damage in sperm and alters testes histopathology in male rats. Toxicol. Lett. 191, 40-46.

Baumann, C.G., Morris, D.G., Sreenan, J.M., Leese, H.J., 2007. The quiet embryo hypothesis: molecular characteristics favoring viability. Mol. Reprod. Dev. 74, 1345-1353.

Beavo, J., 1995. Cyclic nucleotide phosphodiesterases: functional implications of multiple isoforms. Physiol. Revies 75, 725-748.

Benowitz, N.L., 1986. Clinical pharmacology of nicotine. Annu. Rev. Med. 37, 21-32.

Biggers, J.D., Whittingham, D.G., Donahue, R.P., 1967. The pattern of energy metabolism in the mouse oocyte and zygote. Proc. Natl. Acad. Sci. U. S. A. 58, 560-567.

Blanchet, M.-H., Le Good, J.A., Mesnard, D., Oorschot, V., Baflast, S., Minchiotti, G., Klumperman, J., Constam, D.B., 2008. Cripto recruits Furin and PACE4 and controls Nodal trafficking during proteolytic maturation. EMBO J. 27, 2580-2591.

Bodensteiner, K.J., Clay, C.M., Moeller, C.L., Sawyer, H.R., 1999. Molecular cloning of the ovine Growth/Differentiation factor-9 gene and expression of growth/differentiation factor-9 in ovine and bovine ovaries. Biol. Reprod. 60, 381-386. 
Bokkala, S., Reis, H.M., Rubin, E., Joseph, S.K., 2001. Effect of angiotensin II and ethanol on the expression of connexin 43 in WB rat liver epithelial cells. Biochem. J. 357, 769777.

Bolumar, F., Olsen, J., Rebagliato, M., Bisanti, L., 1997. Caffeine intake and delayed conception: a European multicenter study on infertility and subfecundity. Am. J. Epidemiol. 145, 324-334.

Brennan, K.A., Schenk, S., 2006. Initial deficit and recovery of function after MDMA preexposure in rats. Psychopharmacology (Berl.) 184, 239-246.

Brink, P., Cronin, K., Banach, K., Peterson, E., Westphale, E., Seul, K., Ramanan, S., Beyer, E., 1997. Evidence for heteromeric gap junction channels formed from rat connexin43 and human connexin37. Am. J. Physiol.- Cell Physiol. 273, 1386-1396.

Brower, P., Shultz, R., 1982. Intercellular communication between granulosa cells and mouse oocytes: existence and possible nutritional role during oocyte growth. Dev. Biol. 90, 144-153.

Brownlee, M., 2001. Biochemistry and molecular cell biology of diabetic complications. Nature 414, 813-820.

Bruzzone, R., White, T., Paul, D., 1996. Connections with connexins: the molecular basis of direct intercellular signaling. Eur J Biochem 238, 1-27.

Buccione, R., Schroeder, A., Eppig, J., 1990a. Interactions between somatic cells and germ cells throughout mammalian oogenesis. Biol. Reprod. 43, 543-547.

Buccione, R., Vanderhyden, B.C., Caron, P.J., Eppig, J.J., 1990b. FSH-induced expansion of the mouse cumulus oophorus in vitro is dependent upon a specific factor(s) secreted by the oocyte. Dev. Biol. 138, 16-25.

Bukauskas, F., Jordan, K., Bukauskiene, A., Bennett, M., Lampe, P., Laird, W., Verselis, V., 2000. Clustering of connexin 43-enhanced green fluorescent protein gap junction channels and functional coupling in living cells. Proc. Natl. Acad. Sci. U. S. A. 97, 2556-2561.

Bukauskas, F., Verselis, V., 2004. Gap junction channel gating. Biochim. Biophys. Acta BBABiomembr. 1662, 42-60.

Callaway, C.W., Wing, L.L., Geyer, M.A., 1990. Serotonin release contributes to the locomotor stimulant effects of 3,4-methylenedioxymethamphetamine in rats. J. Pharmacol. Exp. Ther. 254, 456-464. 
Cannady, E.A., Dyer, C.A., Christian, P.J., Sipes, I.G., Hoyer, P.B., 2003. Expression and activity of cytochromes P450 2E1, 2A, and $2 \mathrm{~B}$ in the mouse ovary: the effect of 4vinylcyclohexene and its diepoxide metabolite. Toxicol. Sci. Off. J. Soc. Toxicol. 73, 423-430.

Carabatsos, M., Sellitto, C., Goodenough, D., Albertini, D., 2000. Oocyte-granulosa cell heterologous gap junctions are required for the coordination of nuclear and cytoplasmic meiotic competence. Dev. Biol. 226, 167-179.

Cetica, P., Pintos, L., Dalvit, G., Beconi, M., 2002. Activity of key enzymes involved in glucose and triglyceride catabolism during bovine oocyte maturation in vitro. Reproduction $124,675-681$.

Chandra, D., Liu, J.-W., Tang, D.G., 2002. Early mitochondrial activation and cytochrome c upregulation during apoptosis. J. Biol. Chem. 277, 50842-50854.

Chang, A.S., Dale, A.N., Moley, K.H., 2005. Maternal diabetes adversely affects preovulatory oocyte maturation, development, and granulosa cell apoptosis. Endocrinology 146, 2445-2453.

Chavarro, J., Rich-Edwards, J., Rosner, B., Willett, W., 2007. Diet and lifestyle in the prevention of ovulatory disorder infertility. Obstet. Gynecol. 110, 1050-1058.

Cho, W., Stern, S., Biggers, J., 1974. Inhibitory effect of dibutyryl cAMP on mouse oocyte maturation in vitro. J. Exp. Zool. 187, 383-386.

Civitelli, R., Ziambaras, K., Warlow, P.M., Lecanda, F., Nelson, T., Harley, J., Atal, N., Beyer, E.C., Steinberg, T.H., 1998. Regulation of connexin43 expression and function by prostaglandin E2 (PGE2) and parathyroid hormone (PTH) in osteoblastic cells. J. Cell. Biochem. 68, 8-21.

Colonna, R., Mangia, F., 1983. Mechanisms of amino acid uptake in cumulus-enclosed mouse oocytes. Biol. Reprod. 28, 797-803.

Colton, S.A., Downs, S.M., 2004. Potential role for the sorbitol pathway in the meiotic dysfunction exhibited by oocytes from diabetic mice. J. Exp. Zool. 301, 439-448.

Colton, S.A., Humpherson, P.G., Leese, H.J., Downs, S.M., 2003. Physiological changes in oocyte-cumulus cell complexes from diabetic mice that potentially influence meiotic regulation. Biol. Reprod. 69, 761-770.

Conti, M., 2011. Phosphodiesterases and regulation of female reproductive function. Curr. Opin. Pharmacol. 11, 665-669. 
Conti, M., Andersen, C., Richard, F., Mehats, C., Chun, S., Horner, K., Jin, C., Tsafriri, A., 2002. Role of cyclic nucleotide signaling in oocyte maturation. Mol. Cell. Endocrinol. 187, 153-159.

Conti, M., Hsieh, M., Zamah, A., Oh, J., 2012. Novel signaling mechanisms in the ovary during oocyte maturation and ovulation. Mol. Cell. Endocrinol. 356, 65-73.

Coors, A., Jones, P.D., Giesy, J.P., Ratte, H.T., 2003. Removal of estrogenic activity from municipal waste landfill leachate assessed with a bioassay based on reporter gene expression. Environ. Sci. Technol. 37, 3430-3434.

Crawford, J.L., McNatty, K.P., 2012. The ratio of growth differentiation factor 9: bone morphogenetic protein 15 mRNA expression is tightly co-regulated and differs between species over a wide range of ovulation rates. Mol. Cell. Endocrinol. 348, 339-343.

Cruciani, V., Mikalsen, S., 2002. Connexins, gap junctional intercellular communication and kinases. Biol. Cell 94, 433-443.

Dahl, G., Levine, E., Rabadan-Diehl, C., Werner, R., 1991. Cell/cell channel formation involves disulfide exchange. Eur. J. Biochem. 197, 141-144.

Dai, P., Nakagami, T., Tanaka, H., Hitomi, T., Takamatsu, T., 2007. Cx43 Mediates TGF- $\beta$ _ Signaling through Competitive Smads Binding to Microtubules. Mol. Biol. Cell 18, 2264-2273.

De la Torre, R., Farré, M., Ortuño, J., Mas, M., Brenneisen, R., Roset, P.N., Segura, J., Camí, J., 2000. Non-linear pharmacokinetics of MDMA ('ecstasy') in humans. Br. J. Clin. Pharmacol. 49, 104-109.

Diaz, F., Wigglesworth, K., Eppig, J., 2007. Oocytes determine cumulus cell lineage in mouse ovarian follicles. J. Cell Sci. 120, 1330-1340.

Dickerson, S.M., Walker, D.M., Reveron, M.E., Duvauchelle, C.L., Gore, A.C., 2008. The Recreational Drug Ecstasy Disrupts the Hypothalamic-Pituitary-Gonadal Reproductive Axis in Adult Male Rats. Neuroendocrinology 88, 95-102.

Diel, P., Schulz, T., Smolnikar, K., Strunck, E., Vollmer, G., Michna, H., 2000. Ability of xenoand phytoestrogens to modulate expression of estrogen-sensitive genes in rat uterus: estrogenicity profiles and uterotropic activity. J. Steroid Biochem. Mol. Biol. 73, 1-10. 
Domar, A.D., Conboy, L., Denardo-Roney, J., Rooney, K.L., 2012. Lifestyle behaviors in women undergoing in vitro fertilization: a prospective study. Fertil. Steril. 97, 697701.e1.

Dong, J., Albertini, D., Nishimori, K., Kumar, T., Lu, N., Matzuk, M., 1996. Growth differentiation factor-9 is required during early ovarian folliculogenesis. Nature 531535.

Downs, S.M., Humpherson, P.G., Leese, H.J., 1998. Meiotic induction in cumulus cellenclosed mouse oocytes: involvement of the pentose phosphate pathway. Biol. Reprod. 58, 1084-1094.

Downs, S.M., Humpherson, P.G., Leese, H.J., 2002. Pyruvate utilization by mouse oocytes is influenced by meiotic status and the cumulus oophorus. Mol. Reprod. Dev. 62, 113123.

Downs, S.M., Humpherson, P.G., Martin, K.L., Leese, H.J., 1996. Glucose utilization during gonadotropin-induced meiotic maturation in cumulus cell-enclosed mouse oocytes. Mol. Reprod. Dev. 44, 121-131.

Dragovic, R.A., Ritter, L.J., Schulz, S.J., Amato, F., Armstrong, D.T., Gilchrist, R.B., 2005. Role of oocyte-secreted growth differentiation factor 9 in the regulation of mouse cumulus expansion. Endocrinology 146, 2798-2806.

Dragovic, R.A., Ritter, L.J., Schulz, S.J., Amato, F., Thompson, J.G., Armstrong, D.T., Gilchrist, R.B., 2006. Oocyte-Secreted Factor Activation of SMAD 2/3 Signaling Enables Initiation of Mouse Cumulus Cell Expansion. Biol. Reprod. 76, 848-857.

Duffy, D.M., 2003. Growth differentiation factor-9 is expressed by the primate follicle throughout the periovulatory interval. Biol. Reprod. 69, 725-732.

Easton, N., Marsden, C.A., 2006. Ecstasy: are animal data consistent between species and can they translate to humans? J. Psychopharmacol. Oxf. Engl. 20, 194-210.

Eckery, D.C., Whale, L.J., Lawrence, S.B., Wylde, K.A., McNatty, K.P., Juengel, J.L., 2002. Expression of mRNA encoding growth differentiation factor 9 and bone morphogenetic protein 15 during follicular formation and growth in a marsupial, the brushtail possum (Trichosurus vulpecula). Mol. Cell. Endocrinol. 192, 115-126.

Edson, M.A., Nagaraja, A.K., Matzuk, M.M., 2009. The Mammalian Ovary from Genesis to Revelation. Endocr Rev 30, 624-712.

Edwards, R., 1974. Follicular fluid. J. Reprod. Fertil. 37, 189-219. 
Ehrlich, S., Williams, P.L., Hauser, R., Missmer, S.A., Peretz, J., Calafat, A.M., Flaws, J.A., 2013. Urinary bisphenol A concentrations and cytochrome P450 19 A1 (Cyp19) gene expression in ovarian granulosa cells: An in vivo human study. Reprod. Toxicol. Elmsford N 42, 18-23.

Ehrlich, S., Williams, P.L., Missmer, S.A., Flaws, J.A., Ye, X., Calafat, A.M., Petrozza, J.C., Wright, D., Hauser, R., 2012. Urinary bisphenol A concentrations and early reproductive health outcomes among women undergoing IVF. Hum. Reprod. Oxf. Engl. 27, 3583-3592.

Eichenlaub-Ritter, U., Vogt, E., Cukurcam, S., Sun, F., Pacchierotti, F., Parry, J., 2008. Exposure of mouse oocytes to bisphenol A causes meiotic arrest but not aneuploidy. Mutat. Res. Toxicol. Environ. Mutagen. 651, 82-92.

el-Fouly, M., Cook, B., Nekola, M., Nalbandov, A., 1970. Role of the ovum in follicular luteinization. Endocrinology 87, 288-293.

Elfgang, C., Eckert, R., Lichtenberg-Frate, H., Butterweck, A., Traub, O., Klein, R., Hulser, D., Willecke, K., 1995. Specific permeability and selective formation of gap junction channels in connexin-transfected HeLa cells. J. Cell Biol. 129, 805-817.

Elvin, J.A., Clark, A.T., Wang, P., Wolfman, N.M., Matzuk, M.M., 1999. Paracrine actions of growth differentiation factor-9 in the mammalian ovary. Mol. Endocrinol. Baltim. Md 13, 1035-1048.

Emanuele, M.A., Wezeman, F., Emanuele, N.V., 2002. Alcohol's effects on female reproductive function. Alcohol Res. Health 26, 274-281.

Eppig, J., 1996. Coordination of nuclear and cytoplasmic oocyte maturation in eutherian mammals. Reprod. Fertil. Dev. 8, 485-490.

Eppig, J., Wigglesworth, K., Pendola, F., Hirao, Y., 1997. Murine oocytes supress expression of luteinizing hormone receptor messenger ribonucleic acid by granulosa cells. Biol. Reprod. 56, 976-984.

Eppig, J.J., Pendola, F.L., Wigglesworth, K., Pendola, J.K., 2005. Mouse Oocytes Regulate Metabolic Cooperativity Between Granulosa Cells and Oocytes: Amino Acid Transport. Biol. Reprod. 73, 351-357.

Eppig, J.J., Wigglesworth, K., Pendola, F.L., 2002. The mammalian oocyte orchestrates the rate of ovarian follicular development. Proc. Natl. Acad. Sci. U. S. A. 99, 2890-2894. 
Feichtinger, W., Papalambrou, K., Poehl, M., Krischker, U., Neumann, K., 1997. Smoking and in vitro fertilization: a meta-analysis. J. Assist. Reprod. Genet. 14, 596-599.

Feuerstein, P., Cadoret, V., Dalbies-Tran, R., Guerif, F., Bidault, R., Royere, D., 2007. Gene expression in human cumulus cells: one approach to oocyte competence. Hum. Reprod. Oxf. Engl. 22, 3069-3077.

Fitts, R.H., Booth, F.W., Winder, W.W., Holloszy, J.O., 1975. Skeletal muscle respiratory capacity, endurance, and glycogen utilization. Am. J. Physiol. 228, 1029-1033.

FitzHarris, G., Siyanov, V., Baltz, J., 2007. Granulosa cells regulate oocyte intracellular pH against acidosis in preantral follicles by multiple mechanisms. Development 134, 4283-4295.

Flagg-Newton, J.L., Dahl, G., Loewenstein, W.R., 1981. Cell junction and cyclic AMP: 1. Upregulation of junctional membrane permeability and junctional membrane particles by administration of cyclic nucleotide or phosphodiesterase inhibitor. J. Membr. Biol. 63, 105-121.

Fonsart, J., Menet, M.-C., Debray, M., Hirt, D., Noble, F., Scherrmann, J.-M., Declèves, X., 2009. Sprague-Dawley rats display sex-linked differences in the pharmacokinetics of 3,4-methylenedioxymethamphetamine (MDMA) and its metabolite 3,4methylenedioxyamphetamine (MDA). Toxicol. Appl. Pharmacol. 241, 339-347.

Ford, P., Merot, J., Jawerbaum, A., Gimeno, M., Capurro, C., Parisi, M., 2000. Water Permeability in Rat Oocytes at Different Maturity Stages: Aquaporin-9 Expression. J. Membr. Biol. 176, 151-158.

Fujimoto, V.Y., Kim, D., Saal, F.S., Lamb, J.D., Taylor, J.A., Bloom, M.S., 2010. Serum unconjugated bisphenol A concentrations in women may adversely influence oocyte quality during in vitro fertilization. Fertil. Steril. 95, 1816-1819.

Gabbay, K.H., 1975. Hyperglycemia, polyol metabolism, and complications of diabetes mellitus. Annu. Rev. Med. 26, 521-536.

Gaietta, G., Deerinck, T.J., Adams, S.R., Bouwer, J., Tour, O., Laird, D.W., Sosinsky, G.E., Tsien, R.Y., Ellisman, M.H., 2002. Multicolor and electron microscopic imaging of connexin trafficking. Science 296, 503-507.

Galloway, S.M., McNatty, K.P., Cambridge, L.M., Laitinen, M.P., Juengel, J.L., Jokiranta, T.S., McLaren, R.J., Luiro, K., Dodds, K.G., Montgomery, G.W., Beattie, A.E., Davis, G.H., Ritvos, O., 2000. Mutations in an oocyte-derived growth factor gene (BMP15) cause 
increased ovulation rate and infertility in a dosage-sensitive manner. Nat. Genet. 25, 279-283.

Garcia-Cruz, R., Roig, I., Robles, P., Scherthan, H., Garcia Caldés, M., 2009. ATR, BRCA1 and $\mathrm{\gamma H} 2 \mathrm{AX}$ localize to unsynapsed chromosomes at the pachytene stage in human oocytes. Reprod. Biomed. Online 18, 37-44.

Gilchrist, R., Lane, M., Thompson, J., 2008. Oocyte-secreted factors: regulators of cumulus cell function and oocyte quality. Hum. Reprod. Update 14, 159-177.

Gilchrist, R., Ritter, L., Armstrong, D., 2001. Mouse oocyte mitogenic activity is developmentally coordinated throughout folliculogenesis and meiotic maturation. Dev. Biol. 240, 289-298.

Gilchrist, R., Ritter, L., Armstrong, D., 2004a. Oocyte-somatic cell interactions during follicle development in mammals. Anim. Reprod. Sci. 82, 431-446.

Gilchrist, R., Ritter, L., Myllymaa, S., Kaivo-Oja, N., Dragovic, R., Hickey, T., Ritvos, O., Mottershead, D., 2006. Molecular basis of oocyte-paracrine signalling that promotes granulosa cell proliferation. J. Cell Sci. 119, 3811-3821.

Gilchrist, R.B., Ritter, L.J., Cranfield, M., Jeffery, L.A., Amato, F., Scott, S.J., Myllymaa, S., Kaivo-Oja, N., Lankinen, H., Mottershead, D.G., Groome, N.P., Ritvos, O., 2004b. Immunoneutralization of growth differentiation factor 9 reveals it partially accounts for mouse oocyte mitogenic activity. Biol. Reprod. 71, 732-739.

Gill, J., 2000. The effects of moderate alcohol consumption on female hormone levels and reproductive function. Alcohol Alcohol. Oxf. Oxfs. 35, 417-423.

Gilula, N.B., Epstein, M.L., Beers, W.H., 1978. Cell-to-cell communication and ovulation: A study of the cumulus-oocyte complex. J. Cell Biol. 58-75.

Gittens, J., Kidder, G.M., 2005. Differential contributions of connexin37 and connexin43 to oogenesis revealed in chimeric reaggregated mouse ovaries. J. Cell Sci. 118, 50715078.

Glister, C., Groome, N., Knight, P., 2003. Oocyte-Mediated Suppression of Follicle-Stimulating Hormone- and Insulin-Like Growth Factor-Induced Secretion of Steroids and InhibinRelated Proteins by Bovine Granulosa Cells In Vitro: Possible Role of Transforming Growth Factor alpha. Biol. Reprod. 68, 758-765. 
Gode, F., Gulekli, B., Dogan, E., Korham, P., Dogan, S., Bige, O., Cimrin, D., Atabey, N., 2011. Influence of follicular fluid GDF9 and BMP15 on embryo quality. Fertil. Steril. 95, 2274-2278.

Goodenough, D., Paul, D., 2009. Gap junctions. Cold Spring Harb. Perspect. Biol. 1.

Gordon, A.S., Collier, K., Diamond, I., 1986. Ethanol regulation of adenosine receptorstimulated cAMP levels in a clonal neural cell line: an in vitro model of cellular tolerance to ethanol. Proc. Natl. Acad. Sci. U. S. A. 83, 2105-2108.

Gosden, R., Lee, B., 2010. Portrait of an oocyte: our obscure origin. J. Clin. Invest. 120, 973983.

Granot, I., Dekel, N., 1997. Developmental expression and regulation of the gap junction protein and transcript in rat ovaries. Mol. Reprod. Dev. 47, 231-239.

Granot, I., Dekel, N., 2002. The ovarian gap junction protein connexin43: regulation by gonadotropins. Trends Endocrinol. Metab. 13, 310-313.

Green, A.R., Gabrielsson, J., Marsden, C.A., Fone, K.C.F., 2009. MDMA: On the translation from rodent to human dosing. Psychopharmacology (Berl.) 204, 375-378.

Green, A.R., Mechan, A.O., Elliott, J.M., O’Shea, E., Colado, M.I., 2003. The pharmacology and clinical pharmacology of 3,4-methylenedioxymethamphetamine (MDMA, “ecstasy”). Pharmacol. Rev. 55, 463-508.

Greene, S.L., Dargan, P.I., O'Connor, N., Jones, A.L., Kerins, M., 2003. Multiple toxicity from 3, 4-methylenedioxymethamphetamine. Am. J. Emerg. Med. 21, 121-124.

Grotenhermen, F., 2003. Pharmacokinetics and pharmacodynamics of cannabinoids. Clin. Pharmacokinet. 42, 327-360.

Guan, X., Wilson, S., Schlender, K.K., Ruch, R.J., 1996. Gap junction disassembly and connexin 43 dephosphorylation induced by 18 glycyrrhetinic acid. Mol. Carcinog. 16, 157-164.

Gui, L.-M., Joyce, I.M., 2005. RNA interference evidence that growth differentiation factor-9 mediates oocyte regulation of cumulus expansion in mice. Biol. Reprod. 72, 195-199.

Gutnisky, C., Dalvit, G.C., Pintos, L.N., Thompson, J.G., Beconi, M.T., Cetica, P.D., 2007. Influence of hyaluronic acid synthesis and cumulus mucification on bovine oocyte in vitro maturation, fertilisation and embryo development. Reprod. Fertil. Dev. 19, 488497.

Gygi, S.P., Rochon, Y., Franza, B.R., Aebersold, R., 1999. Correlation between protein and mRNA abundance in yeast. Mol. Cell. Biol. 19, 1720-1730. 
Haghighat, N., Van Winkle, L., 1990. Developmental change in follicular cell-enhanced amino acid uptake into mouse oocytes that depends on intact gap junctions and transport system Gly. J. Exp. Zool. 253, 71-82.

Hanrahan, J.P., Gregan, S.M., Mulsant, P., Mullen, M., Davis, G.H., Powell, R., Galloway, S.M., 2004. Mutations in the genes for oocyte-derived growth factors GDF9 and BMP15 are associated with both increased ovulation rate and sterility in Cambridge and Belclare sheep (Ovis aries). Biol. Reprod. 70, 900-909.

Hardy, K., Hooper, M.A., Handyside, A.H., Rutherford, A.J., Winston, R.M., Leese, H.J., 1989. Non-invasive measurement of glucose and pyruvate uptake by individual human oocytes and preimplantation embryos. Hum. Reprod. Oxf. Engl. 4, 188-191.

Harris, A.L., 2002. Voltage-sensing and Substate Rectification. J. Gen. Physiol. 119, 165-169.

Harris, S.E., Leese, H.J., Gosden, R.G., Picton, H.M., 2009. Pyruvate and oxygen consumption throughout the growth and development of murine oocytes. Mol. Reprod. Dev. 76, 231-238.

Harrison, C.A., Al-Musawi, S.L., Walton, K.L., 2011. Prodomains regulate the synthesis, extracellular localisation and activity of TGF- $\beta$ superfamily ligands. Growth Factors Chur Switz. 29, 174-186.

Hashimoto, S., Minami, N., Yamada, M., Imai, H., 2000. Excessive concentration of glucose during in vitro maturation impairs the developmental competence of bovine oocytes after in vitro fertilization: relevance to intracellular reactive oxygen species and glutathione contents. Mol. Reprod. Dev. 56, 520-526.

Hasthorpe, S., Tainton, K., Peart, M., Roeszler, K.N., Bell, K.M., Lusby, P.E., Hutson, J.M., Tymms, M.J., 2007. G2/M checkpoint gene expression in developing germ cells. Mol. Reprod. Dev. 74, 531-538.

Hatch, E.E., Bracken, M.B., 1993. Association of delayed conception with caffeine consumption. Am. J. Epidemiol. 138, 1082-1092.

Hayashi, M., McGee, E.A., Min, G., Klein, C., Rose, U.M., van Duin, M., Hsueh, A.J., 1999. Recombinant growth differentiation factor-9 (GDF-9) enhances growth and differentiation of cultured early ovarian follicles. Endocrinology 140, 1236-1244.

Hérubel, F., El Mouatassim, S., Guérin, P., Frydman, R., Ménézo, Y., 2002. Genetic expression of monocarboxylate transporters during human and murine oocyte maturation and early embryonic development. Zygote Camb. Engl. 10, 175-181. 
Hervé, J.-C., Derangeon, M., Bahbouhi, B., Mesnil, M., Sarrouilhe, D., 2007. The Connexin Turnover, an Important Modulating Factor of the Level of Cell-to-Cell Junctional Communication: Comparison with Other Integral Membrane Proteins. J. Membr. Biol. 217, 21-33.

Hickey, T., Marrocco, D., Amato, F., Ritter, L., Norman, R., Gilchrist, R., Armstrong, D., 2005. Androgens augment the mitogenic effects of oocyte-secreted factors and growth differentiation factor 9 on porcine granulosa cells. Biol. Reprod. 73, 825-832.

Himelstein-Braw, R., Peters, H., Faber, M., 1978. Morphological study of the ovaries of leukaemic children. Br. J. Cancer 38, 82-87.

Hiroi, T., Imaoka, S., Chow, T., Funae, Y., 1998. Tissue distributions of CYP2D1, 2D2, 2D3 and 2D4 mRNA in rats detected by RT-PCR. Biochim. Biophys. Acta 1380, 305-312.

Hirshfield, A.N., 1991. Development of follicles in the mammalian ovary. Int. Rev. Cytol. 124, 43-101.

Hukkanen, J., Jacob, P., 3rd, Benowitz, N.L., 2005. Metabolism and disposition kinetics of nicotine. Pharmacol. Rev. 57, 79-115.

Hussein, T., Thompson, J., Gilchrist, R., 2006. Oocyte-secreted factors enhance oocyte developmental competence. Dev. Biol. 296, 514-521.

Hussein, T.S., Froiland, D.A., Amato, F., Thompson, J.G., Gilchrist, R.B., 2005. Oocytes prevent cumulus cell apoptosis by maintaining a morphogenic paracrine gradient of bone morphogenetic proteins. J. Cell Sci. 118, 5257-5268.

Ikezuki, Y., Tsutsumi, O., Takai, Y., Kamei, Y., Taketani, Y., 2002. Determination of bisphenol A concentrations in human biological fluids reveals significant early prenatal exposure. Hum. Reprod. 17, 2839-2841.

Jaatinen, R., Laitinen, M., Vuojolainen, K., Aaltonen, J., Louhio, H., Heikinheimo, K., Lehtonen, E., Ritvos, O., 1999. Localization of growth differentiation factor-9 (GDF-9) mRNA and protein in rat ovaries and CDNA cloning of rat GDF-9 and its novel homolog GDF-9B. Mol. Cel 156, 189-193.

Jagiello, G., Ducayen, M., Lin, J.S., 1972. Meiosis suppression by caffeine in female mice. Mol. Gen. Genet. MGG 118, 209-214.

Johnson, J., Canning, J., Kaneko, T., Pru, J.K., Tilly, J.L., 2004. Germline stem cells and follicular renewal in the postnatal mammalian ovary. Nature 428, 145-150. 
Jordan, K., Chodock, R., Hand, A.R., Laird, D.W., 2001. The origin of annular junctions: a mechanism of gap junction internalization. J. Cell Sci. 114, 763-773.

Juengel, J., McNatty, K., 2005. The role of proteins of the transforming growth factor- $\beta$ superfamily in the intraovarian regulation of follicular development. Hum. Reprod. Update 11, 144-161.

Kadle, R., Zhang, J.T., Nicholson, B.J., 1991. Tissue-specific distribution of differentially phosphorylated forms of Cx43. Mol. Cell. Biol. 11, 363-369.

Kaufman, D.W., Slone, D., Rosenberg, L., Miettinen, O.S., Shapiro, S., 1980. Cigarette smoking and age at natural menopause. Am. J. Public Health 70, 420-422.

Kidder, G., Mhawi, A., 2002. Gap junctions and ovarian folliculogenesis. Reproduction 123, 613-620.

Kidder, G., Vanderhyden, B., 2010. Bidirectional communication between oocytes and follicle cells: ensuring oocyte developmental competence. Can. J. Physiol. Pharmacol. 88, 399-413.

Kim, K., Kim, C.H., Moley, K.H., Cheon, Y.-P., 2007. Disordered meiotic regulation of oocytes by duration of diabetes mellitus in BBdp rat. Reprod. Sci. Thousand Oaks Calif 14, 467-474.

Klonoff-Cohen, H., 2005. Female and male lifestyle habits and IVF: what is known and unknown. Hum Reprod Update 11, 180-204.

Klonoff-Cohen, H., Bleha, J., Lam-Kruglick, P., 2002. A prospective study of the effects of female and male caffeine consumption on the reproductive endpoints of IVF and gamete intra-Fallopian transfer. Hum. Reprod. Oxf. Engl. 17, 1746-1754.

Klonoff-Cohen, H.S., Natarajan, L., Victoria Chen, R., 2006. A prospective study of the effects of female and male marijuana use on in vitro fertilization (IVF) and gamete intrafallopian transfer (GIFT) outcomes. Am. J. Obstet. Gynecol. 194, 369-376.

Knight, P., Glister, C., 2006. TGF- $\beta$ superfamily members and ovarian follicle development. Reproduction 132, 191-206.

Kobayashi, T., Gamanuma, M., Sasaki, T., Yamashita, Y., Yuasa, K., Kotera, J., Omori, K., 2003. Molecular comparison of rat cyclic nucleotide phosphodiesterase 8 family: unique expression of PDE8B in rat brain. Gene 319, 21-31. 
Kogo, H., Tsutsumi, M., Inagaki, H., Ohye, T., Kiyonari, H., Kurahashi, H., 2012. HORMAD2 is essential for synapsis surveillance during meiotic prophase via the recruitment of ATR activity. Genes Cells Devoted Mol. Cell. Mech. 17, 897-912.

Kren, R., Ogushi, S., Miyano, T., 2004. Effect of caffeine on meiotic maturation of porcine oocytes. Zygote Camb. Engl. 12, 31-38.

Krisher, R.L., 2004. The effect of oocyte quality on development. J Anim Sci 82, E14-23.

Krisher, R.L., Bavister, B.D., 1999. Enhanced glycolysis after maturation of bovine oocytes in vitro is associated with increased developmental competence. Mol. Reprod. Dev. 53, 19-26.

Kumar, T.R., Wang, Y., Lu, N., Matzuk, M.M., 1997. Follicle stimulating hormone is required for ovarian follicle maturation but not male fertility. Nat. Genet. 15, 201-204.

Laitinen, M., Vuojolainen, K., Jaatinen, R., Ketola, I., Aaltonen, J., Lehtonen, E., Heikinheimo, M., Ritvos, O., 1998. A novel growth differentiation factor-9 (GDF-9) related factor is co-expressed with GDF-9 in mouse oocytes during folliculogenesis. Mech. Dev. 78, 135-140.

Lampe, P.D., Cooper, C.D., King, T.J., Burt, J.M., 2006. Analysis of Connexin43 phosphorylated at S325, S328 and S330 in normoxic and ischemic heart. J. Cell Sci. 119, 3435-3442.

Lampe, P.D., Lau, A.F., 2000. Regulation of gap junctions by phosphorylation of connexins. Arch. Biochem. Biophys. 384, 205-215.

Lampe, P.D., TenBroek, E.M., Burt, J.M., Kurata, W.E., Johnson, R.G., Lau, A.F., 2000. Phosphorylation of connexin43 on serine 368 by protein kinase $C$ regulates gap junctional communication. J. Cell Biol. 149, 1503-1512.

Lasien, K., Vitkus, A., Valan i t, A., Lasys, V., Skeberdis, V., Gendvilien, V., Zablockait, D., Martišien, I., Stankevi ius, A., Goštautas, A., 2009. Morphological criteria of oocyte quality. Med. Kaunas 45, 509-515.

Lee, I.K., Rhee, S.K., 2007. Inhibitory effect of bisphenol a on gap junctional intercellular communication in an epithelial cell line of rat mammary tissue. Arch. Pharm. Res. 30, 337-343.

Lee, S.Y., Oh, S.J., Yun, K.U., Kim, H.M., Kim, B.-H., Lee, K., Kim, S.K., 2012. Expression of hepatic and ovarian cytochrome P450 during estrous cycle in rats. Arch. Toxicol. 86, 75-85. 
Lemberger, L., Martz, R., Rodda, B., Forney, R., Rowe, H., 1973. Comparative pharmacology of $\Delta 9$-tetrahydrocannabinol and its metabolite, $11-\mathrm{OH}-\Delta 9$-tetrahydrocannabinol. J. Clin. Invest. 52, 2411-2417.

Lenie, S., Cortvrindt, R., Eichenlaub-Ritter, U., Smitz, J., 2008. Continuous exposure to bisphenol A during in vitro follicular development induces meiotic abnormalities. Mutat. Res. Toxicol. Environ. Mutagen. 651, 71-81.

Leviton, A., Cowan, L., 2002. A review of the literature relating caffeine consumption by women to their risk of reproductive hazards. Food Chem. Toxicol. 40, 1271-1310.

Li, R., Norman, R., Armstrong, D., Gilchrist, R., 2000. Oocyte-secreted factor (s) determine functional differences between bovine mural granulosa cells and cumulus cells. Biol. Reprod. 63, 839-845.

Lin, J.Y., Pitman-Crawford, J.L., Bibby, A.H., Hudson, N.L., McIntosh, C.J., Juengel, J.L., McNatty, K.P., 2012. Effects of species differences on oocyte regulation of granulosa cell function. Reprod. Camb. Engl. 144, 557-567.

Lindgren, J.E., Ohlsson, A., Agurell, S., Hollister, L., Gillespie, H., 1981. Clinical effects and plasma levels of $\Delta 9$-tetrahydrocannabinol $(\Delta 9-T H C)$ in heavy and light users of cannabis. Psychopharmacology (Berl.) 74, 208-212.

Liu, Y., Li, G., Rickords, L., White, K., Sessions, B., Aston, K., Bunch, T., 2008. Effect of nicotine on in vitro maturation of bovine oocytes. Anim. Reprod. Sci. 103, 13-24.

Liu, Y., Li, G.P., White, K.L., Rickords, L.F., Sessions, B.R., Aston, K.I., Bunch, T.D., 2007. Nicotine alters bovine oocyte meiosis and affects subsequent embryonic development. Mol. Reprod. Dev. 74, 1473-1482.

Livak, K.J., Schmittgen, T.D., 2001. Analysis of Relative Gene Expression Data Using Real-Time Quantitative PCR and the 2- $\Delta \Delta C T$ Method. Methods 25, 402-408.

Lynskey, M.T., Fergusson, D.M., Horwood, L.J., 1998. The origins of the correlations between tobacco, alcohol, and cannabis use during adolescence. J. Child Psychol. Psychiatry 39, 995-1005.

Macaldowie, A., Wang, Y., Chambers, G., Sullivan, E., 2012. Assisted reproductive thechnology in Australia and New Zealand 2010 ( No. 16), Assisted reproduction technology series. AlHW, Canberra. 
Machtinger, R., Combelles, C.M.H., Missmer, S.A., Correia, K.F., Williams, P., Hauser, R., Racowsky, C., 2013. Bisphenol-A and human oocyte maturation in vitro. Hum. Reprod. Oxf. Engl. 28, 2735-2745.

Maeda, S., Nakagawa, S., Suga, M., Yamashita, E., Oshima, A., Fujiyoshi, Y., Tsukihara, T., 2009. Structure of the connexin 26 gap junction channel at $3.5 \AA$ resolution. Nature 458, 597-602.

Magoffin, D.A., 2005. Ovarian theca cell. Int. J. Biochem. Cell Biol. 37, 1344-1349.

Mailhes, J.B., Young, D., Caldito, G., London, S., 2000. Sensitivity of mouse oocytes to nicotine-induced perturbations during oocyte meiotic maturation and aneuploidy in vivo and in vitro. Mol. Hum. Reprod. 6, 232-237.

Marangos, P., Carroll, J., 2012. Oocytes progress beyond prophase in the presence of DNA damage. Curr. Biol. CB 22, 989-994.

Marteil, G., Richard-Parpaillon, L., Kubiak, J., 2009. Role of oocyte quality in meiotic maturation and embryonic development. Reprod Biol 9, 203-24.

Mattison, D.R., Thorgeirsson, S.S., 1979. Ovarian aryl hydrocarbon hydroxylase activity and primordial oocyte toxicity of polycyclic aromatic hydrocarbons in mice. Cancer Res. 39, 3471-3475.

Mazerbourg, S., Klein, C., Roh, J., Kaivo-Oja, N., Mottershead, D.G., Korchynskyi, O., Ritvos, O., Hsueh, A.J.W., 2004. Growth differentiation factor-9 signaling is mediated by the type I receptor, activin receptor-like kinase 5. Mol. Endocrinol. Baltim. Md 18, 653665.

McCann, U.D., Ricaurte, G.A., 2001. Caveat emptor: editors beware. Neuropsychopharmacol. Off. Publ. Am. Coll. Neuropsychopharmacol. 24, 333-336.

McGee, E., Hsueh, A., 2000. Initial and cyclic recruitment of ovarian follicles. Endocr. Rev. 21, 200-214.

McIntosh, C.J., Lawrence, S., Smith, P., Juengel, J.L., McNatty, K.P., 2012. Active immunization against the proregions of GDF9 or BMP15 alters ovulation rate and litter size in mice. Reprod. Camb. Engl. 143, 195-201.

McIntosh, C.J., Lun, S., Lawrence, S., Western, A.H., McNatty, K.P., Juengel, J.L., 2008. The Proregion of Mouse BMP15 Regulates the Cooperative Interactions of BMP15 and GDF9. Biol. Reprod. 79, 889-896. 
McMahon, H.E., Sharma, S., Shimasaki, S., 2008. Phosphorylation of bone morphogenetic protein-15 and growth and differentiation factor-9 plays a critical role in determining agonistic or antagonistic functions. Endocrinology 149, 812-817.

McNatty, K.P., 2000. Ovarian Development: Fetus to Puberty. In: Salamonsen, L.A. (Ed.), Hormones and Women's Health. Harwood Academinc Publishers, The Netherland, pp. 9-22.

McNatty, K.P., Heath, D., Hudson, NL, Reader, K.L., Quirke, L, Lun, S, Juengel, J., 2010. The conflict between hierarchical ovarian follicular development and superovulation treatment. Reproduction 140, 287-294.

McNatty, K.P., Juengel, J.L., Reader, K.L., Lun, S., Myllymaa, S., Lawrence, S.B., Western, A., Meerasahib, M.F., Mottershead, D.G., Groome, N.P., 2005a. Bone morphogenetic protein 15 and growth differentiation factor 9 co-operate to regulate granulosa cell function. Reproduction 129, 473-480.

McNatty, K.P., Juengel, J.L., Reader, K.L., Lun, S., Myllymaa, S., Lawrence, S.B., Western, A., Meerasahib, M.F., Mottershead, D.G., Groome, N.P., Ritvos, O., Laitinen, M.P.E., 2005b. Bone morphogenetic protein 15 and growth differentiation factor 9 cooperate to regulate granulosa cell function in ruminants. Reprod. Camb. Engl. 129, 481-487.

McNatty, K.P., Lawrence, S., Groome, N.P., Meerasahib, M.F., Hudson, N.L., Whiting, L., Heath, D.A., Juengel, J.L., 2006. Meat and Livestock Association Plenary Lecture 2005. Oocyte signalling molecules and their effects on reproduction in ruminants. Reprod. Fertil. Dev. 18, 403-412.

Mehlmann, L., 2005. Stops and starts in mammalian oocytes: recent advances in understanding the regulation of meiotic arrest and oocyte maturation. Reproduction 130, 791-799.

Mehta, P.P., Yamamoto, M., Rose, B., 1992. Transcription of the gene for the gap junctional protein connexin43 and expression of functional cell-to-cell channels are regulated by cAMP. Mol. Biol. Cell 3, 839-850.

Mendelson, J.H., Lukas, S.E., Mello, N.K., Amass, L., Ellingboe, J., Skupny, A., 1988. Acute alcohol effects on plasma estradiol levels in women. Psychopharmacology (Berl.) 94, 464-467. 
Mendelson, J.H., Mello, N.K., 1988. Chronic alcohol effects on anterior pituitary and ovarian hormones in healthy women. J. Pharmacol. Exp. Ther. 245, 407-412.

Mendelson, J.H., Mello, N.K., Ellingboe, J., Skupny, A.S., Lex, B.W., Griffin, M., 1986. Marihuana smoking suppresses luteinizing hormone in women. J. Pharmacol. Exp. Ther. 237, 862-866.

Messing, R.O., Carpenter, C.L., Diamond, I., Greenberg, D.A., 1986. Ethanol regulates calcium channels in clonal neural cells. Proc. Natl. Acad. Sci. U. S. A. 83, 6213-6215.

Miles, D., van den Bergen,, J., Sinclair,, A., Western,, P., 2010. Regulation of the female mouse germ cell cycle during entry into meiosis. Cell Cycle 9, 408-418.

Mochly-Rosen, D., Chang, F.H., Cheever, L., Kim, M., Diamond, I., Gordon, A.S., 1988. Chronic ethanol causes heterologous desensitization of receptors by reducing alpha $\mathrm{S}$ messenger RNA. Nature 333, 848-850.

Mohri, T., Yoshida, S., 2005. Estrogen and bisphenol A disrupt spontaneous $[\mathrm{Ca}(2+)](\mathrm{i})$ oscillations in mouse oocytes. Biochem. Biophys. Res. Commun. 326, 166-173.

Mok-Lin, E., Ehrlich, S., Williams, P.L., Petrozza, J., Wright, D.L., Calafat, A.M., Ye, X., Hauser, R., 2010. Urinary bisphenol A concentrations and ovarian response among women undergoing IVF. Int. J. Androl. 33, 385-393.

Moreno, A., 2005. Connexin phosphorylation as a regulatory event linked to channel gating. BBA-Biomembr. 1711, 164-171.

Mottershead, D.G., Harrison, C.A., Mueller, T.D., Stanton, P.G., Gilchrist, R.B., McNatty, K.P., 2013. Growth differentiation factor 9:bone morphogenetic protein 15 (GDF9:BMP15) synergism and protein heterodimerization. Proc. Natl. Acad. Sci. U. S. A. 110, E2257.

Mottershead, D.G., Ritter, L.J., Gilchrist, R.B., 2012. Signalling pathways mediating specific synergistic interactions between GDF9 and BMP15. Mol. Hum. Reprod. 18, 121-128.

Moyer, T.P., Charlson, J.R., Enger, R.J., Dale, L.C., Ebbert, J.O., Schroeder, D.R., Hurt, R.D., 2002. Simultaneous analysis of nicotine, nicotine metabolites, and tobacco alkaloids in serum or urine by tandem mass spectrometry, with clinically relevant metabolic profiles. Clin. Chem. 48, 1460-1471.

Musil, L.S., Goodenough, D.A., 1993. Multisubunit assembly of an integral plasma membrane channel protein, gap junction connexin43, occurs after exit from the ER. Cell 74, 1065-1077. 
Mustonen, H., Kiviluoto, T., Paimela, H., Puolakkainen, P., Kivilaakso, E., 2005. Calcium signaling is involved in ethanol-induced volume decrease and gap junction closure in cultured rat gastric mucosal cells. Dig. Dis. Sci. 50, 103-110.

Nakajo, N., Oe, T., Uto, K., Sagata, N., 1999. Involvement of Chk1 kinase in prophase I arrest of Xenopus oocytes. Dev. Biol. 207, 432-444.

Navot, D., Bergh, P.A., Williams, M.A., Garrisi, G.J., Guzman, I., Sandler, B., Grunfeld, L., 1991. Poor oocyte quality rather than implantation failure as a cause of age-related decline in female fertility. Lancet 337, 1375-1377.

Nekola, M., Nalbandov, A., 1971. Morphological changes of rat follicular cells as influenced by oocytes. Biol. Reprod. 4, 154-160.

Nishimoto, H., Matsutani, R., Yamamoto, S., Takahashi, T., Hayashi, K.-G., Miyamoto, A., Hamano, S., Tetsuka, M., 2006. Gene expression of glucose transporter (GLUT) 1, 3 and 4 in bovine follicle and corpus luteum. J. Endocrinol. 188, 111-119.

Nogueira, D., Ron-El, R., Friedler, S., Schachter, M., Raziel, A., Cortvrindt, R., Smitz, J., 2006. Meiotic arrest in vitro by phosphodiesterase 3-inhibitor enhances maturation capacity of human oocytes and allows subsequent embryonic development. Biol. Reprod. 74, 177-184.

Norris, R.P., Freudzon, M., Mehlmann, L.M., Cowan, A.E., Simon, A.M., Paul, D.L., Lampe, P.D., Jaffe, L.A., 2008. Luteinizing hormone causes MAP kinase-dependent phosphorylation and closure of connexin 43 gap junctions in mouse ovarian follicles: one of two paths to meiotic resumption. Development 135, 3229-3238.

Norris, R.P., Ratzan, W.J., Freudzon, M., Mehlmann, L.M., Krall, J., Movsesian, M.A., Wang, H., Ke, H., Nikolaev, V.O., Jaffe, L.A., 2009. Cyclic GMP from the surrounding somatic cells regulates cyclic AMP and meiosis in the mouse oocyte. Development 136, 18691878.

O'Neill, G.T., McDougall, R.D., Kaufman, M.H., 1989. Ultrastructural analysis of abnormalities in the morphology of the second meiotic spindle in ethanol-induced parthenogenones. Gamete Res. 22, 285-299.

Obrocki, J., Buchert, R., Väterlein, O., Thomasius, R., Beyer, W., Schiemann, T., 1999. Ecstasylong-term effects on the human central nervous system revealed by positron emission tomography. Br. J. Psychiatry J. Ment. Sci. 175, 186-188. 
Otsuka, F., Yamamoto, S., Erickson, G.F., Shimasaki, S., 2001. Bone morphogenetic protein15 inhibits follicle-stimulating hormone (FSH) action by suppressing FSH receptor expression. J. Biol. Chem. 276, 11387-11392.

Otsuka, F., Yao, Z., Lee, T., Yamamoto, S., Erickson, G.F., Shimasaki, S., 2000. Bone morphogenetic protein-15. Identification of target cells and biological functions. J. Biol. Chem. 275, 39523-39528.

Paradis, F., Novak, S., Murdoch, G.K., Dyck, M.K., Dixon, W.T., Foxcroft, G.R., 2009. Temporal regulation of BMP2, BMP6, BMP15, GDF9, BMPR1A, BMPR1B, BMPR2 and TGFBR1 mRNA expression in the oocyte, granulosa and theca cells of developing preovulatory follicles in the pig. Reprod. Camb. Engl. 138, 115-129.

Park, B., McPartland, J., Glass, M., 2004. Cannabis, cannabinoids and reproduction. Prostaglandins Leukot. Essent. Fatty Acids 70, 189-197.

Park, J.-Y., Richard, F., Chun, S.-Y., Park, J.-H., Law, E., Horner, K., Jin, S.-L.C., Conti, M., 2003. Phosphodiesterase regulation is critical for the differentiation and pattern of gene expression in granulosa cells of the ovarian follicle. Mol. Endocrinol. Baltim. Md 17, 1117-1130.

Patel, S., 2009. Development of an in vitro assay to assess gap junction activity in cumulusoocyte complexes (COC) in the rat. VUW Masters Thesis.

Peng, J., Li, Q., Wigglesworth, K., Rangarajan, A., Kattamuri, C., Peterson, R.T., Eppig, J.J., Thompson, T.B., Matzuk, M.M., 2013. Growth differentiation factor 9:bone morphogenetic protein 15 heterodimers are potent regulators of ovarian functions. Proc. Natl. Acad. Sci. U. S. A. 110, E776-785.

Peracchia, C., 2004. Chemical gating of gap junction channels:: Roles of calcium, pH and calmodulin. Biochim. Biophys. Acta BBA-Biomembr. 1662, 61-80.

Pfeifer, I., Anderson, C., Werner, R., Oltra, E., 2004. Redefining the structure of the mouse connexin43 gene: selective promoter usage and alternative splicing mechanisms yield transcripts with different translational efficiencies. Nucleic Acids Res. 32, 4550-4562.

Pichini, S., De Luca, R., Pellegrini, M., Marchei, E., Rotolo, M.C., Spoletini, R., D’Aloja, P., Pacifici, R., Mortali, C., Scaravelli, G., 2012. Hair and urine testing to assess drugs of abuse consumption in couples undergoing assisted reproductive technology (ART). Forensic Sci. Int. 218, 57-61. 
Pincus, G., Enzmann, E., 1935. The comparative behavior of mammalian eggs in vivo and in vitro. J. Exp. Med. 62, 665-675.

Pisani, L.F., Antonini, S., Pocar, P., Ferrari, S., Brevini, T.A.L., Rhind, S.M., Gandolfi, F., 2008. Effects of pre-mating nutrition on mRNA levels of developmentally relevant genes in sheep oocytes and granulosa cells. Reprod. Camb. Engl. 136, 303-312.

Purcell, S.H., Moley, K.H., 2009. Glucose transporters in gametes and preimplantation embryos. Trends Endocrinol. Metab. 20, 483-489.

Racowsky, C., Baldwin, K., Larabell, C., DeMarais, A., Kazilek, C., 1989. Down-regulation of membrana granulosa cell gap junctions is correlated with irreversibly commitment to resume meiosis in golden Syrian hamster oocytes. Eur. J. Cell Biol. 49, 244-251.

Rahimi, R., Ghiasi, S., Azimi, H., Fakhari, S., Abdollahi, M., 2010. A review of the herbal phosphodiesterase inhibitors; future perspective of new drugs. Cytokine 49, $123-$ 129.

Rajah, R., Glaser, E.M., Hirshfield, A.N., 1992. The changing architecture of the neonatal rat ovary during histogenesis. Dev. Dyn. Off. Publ. Am. Assoc. Anat. 194, 177-192.

Ratchford, A.M., Esguerra, C.R., Moley, K.H., 2008. Decreased Oocyte-Granulosa Cell Gap Junction Communication and Connexin Expression in a Type 1 Diabetic Mouse Model. Mol. Endocrinol. 22, 2643-2654.

Reader, K.L., Heath, D.A., Lun, S., McIntosh, C.J., Western, A.H., Littlejohn, R.P., McNatty, K.P., Juengel, J.L., 2011. Signalling pathways involved in the cooperative effects of ovine and murine GDF9+BMP15-stimulated thymidine uptake by rat granulosa cells. Reprod. Camb. Engl. 142, 123-131.

Reich, R., Laufer, N., Lewysohn, O., Cordova, T., Ayalon, D., Tsafriri, A., 1982. In vitro effects of cannabinoids on follicular function in the rat. Biol. Reprod. 27, 223-231.

Rhind, N., 2009. Changing of the guard: how ATM hands off DNA double-strand break signaling to ATR. Mol. Cell 33, 672-674.

Richard, F., Tsafriri, A., Conti, M., 2001. Role of Phosphodiesterase Type 3A in Rat Oocyte Maturation. Biol. Reprod. 65, 1444-1451.

Rivedal, E., Mollerup, S., Haugen, A., Vikhamar, G., 1996. Modulation of gap junctional intercellular communication by EGF in human kidney epithelial cells. Carcinogenesis $17,2321-2328$. 
Roberts, R., Stark, J., latropoulou, A., Becker, D.L., Franks, S., Hardy, K., 2004. Energy substrate metabolism of mouse cumulus-oocyte complexes: response to folliclestimulating hormone is mediated by the phosphatidylinositol 3-kinase pathway and is associated with oocyte maturation. Biol. Reprod. 71, 199-209.

Robinson, J.W., Zhang, M., Shuhaibar, L.C., Norris, R.P., Geerts, A., Wunder, F., Eppig, J.J., Potter, L.R., Jaffe, L.A., 2012. Luteinizing hormone reduces the activity of the NPR2 guanylyl cyclase in mouse ovarian follicles, contributing to the cyclic GMP decrease that promotes resumption of meiosis in oocytes. Dev. Biol. 366, 308-316.

Roh, J.-S., Bondestam, J., Mazerbourg, S., Kaivo-Oja, N., Groome, N., Ritvos, O., Hsueh, A.J.W., 2003. Growth differentiation factor-9 stimulates inhibin production and activates Smad2 in cultured rat granulosa cells. Endocrinology 144, 172-178.

Rossetti, L., 2000. Perspective: Hexosamines and nutrient sensing. Endocrinology 141, 19221925.

Rout, U.K., Armant, D.R., 2002. Expression of genes for alcohol and aldehyde metabolizing enzymes in mouse oocytes and preimplantation embryos. Reprod. Toxicol. Elmsford N 16, 253-258.

Russell, D., Robker, R., 2007. Molecular mechanisms of ovulation: co-ordination through the cumulus complex. Hum. Reprod. Update 13, 289-312.

Russell, M., Jarvis, M., Iyer, R., Feyerabend, C., 1980. Relation of nicotine yield of cigarettes to blood nicotine concentrations in smokers. Br. Med. J. 280, 972-976.

Saeki, K., Nagao, Y., Kishi, M., Nagai, M., 1997. Developmental capacity of bovine oocytes following inhibition of meiotic resumption by cycloheximide or 6dimethylaminopurine. Theriogenology 48, 1161-1172.

Saito, T., Hiroi, M., Kato, T., 1994. Development of glucose utilization studied in single oocytes and preimplantation embryos from mice. Biol. Reprod. 50, 266-270.

Salhab, M., Dhorne-Pollet, S., Auclair, S., Guyader-Joly, C., Brisard, D., Dalbies-Tran, R., Dupont, J., Ponsart, C., Mermillod, P., Uzbekova, S., 2012. In vitro maturation of oocytes alters gene expression and signaling pathways in bovine cumulus cells. Mol. Reprod. Dev. 80, 166-182.

Sasseville, M., Albuz, F.K., Cote, N., Guillemette, C., Gilchrist, R.B., Richard, F.J., 2009a. Characterization of Novel Phosphodiesterases in the Bovine Ovarian Follicle. Biol. Reprod. 81, 415-425. 
Sasseville, M., Gagnon, M.-C., Guillemette, C., Sullivan, R., Gilchrist, R.B., Richard, F.J., 2009b. Regulation of Gap Junctions in Porcine Cumulus-Oocyte Complexes: Contributions of Granulosa Cell Contact, Gonadotropins, and Lipid Rafts. Mol. Endocrinol. 23, 700710.

Sawyer, H.R., Smith, P., Heath, D.A., Juengel, J.L., Wakefield, S.J., McNatty, K.P., 2002. Formation of ovarian follicles during fetal development in sheep. Biol. Reprod. 66, 1134-1150.

Schelbach, C.J., Kind, K.L., Lane, M., Thompson, J.G., 2010. Mechanisms contributing to the reduced developmental competence of glucosamine-exposed mouse oocytes. Reprod. Fertil. Dev. 22, 771-779.

Schenk, S., 2009. MDMA self-administration in laboratory animals: a summary of the literature and proposal for future research. Neuropsychobiology 60, 130-136.

Segretain, D., Falk, M., 2004. Regulation of connexin biosynthesis, assembly, gap junction formation, and removal. Biochim. Biophys. Acta BBA-Biomembr. 1662, 3-21.

Sela-Abramovich, S., 2006. Disruption of Gap Junctional Communication within the Ovarian Follicle Induces Oocyte Maturation. Endocrinology 147, 2280-2286.

Sengle, G., Charbonneau, N.L., Ono, R.N., Sasaki, T., Alvarez, J., Keene, D.R., Bächinger, H.P., Sakai, L.Y., 2008. Targeting of bone morphogenetic protein growth factor complexes to fibrillin. J. Biol. Chem. 283, 13874-13888.

Shiloh, H., Lahav-Baratz, S., Koifman, M., Ishai, D., Bidder, D., Weiner-Meganzi, Z., Dirnfeld, M., 2004. The impact of cigarette smoking on zona pellucida thickness of oocytes and embryos prior to transfer into the uterine cavity. Hum. Reprod. Oxf. Engl. 19, 157159.

Shimada, M., Maeda, T., Terada, T., 2001. Dynamic changes of connexin-43, gap junctional protein, in outer layers of cumulus cells are regulated by PKC and PI 3-kinase during meiotic resumption in porcine oocytes. Biol. Reprod. 64, 1255-1263.

Shimada, M., Terada, T., 2001. Phosphatidylinositol 3-Kinase in Cumulus Cells and Oocytes Is Responsible for Activation of Oocyte Mitogen-Activated Protein Kinase During Meiotic Progression Beyond the Meiosis I Stage in Pigs. Biol. Reprod. 64, 1106-1114.

Shimada, T., Yamazaki, H., Mimura, M., Inui, Y., Guengerich, F.P., 1994. Interindividual variations in human liver cytochrome P-450 enzymes involved in the oxidation of 
drugs, carcinogens and toxic chemicals: studies with liver microsomes of 30 Japanese and 30 Caucasians. J. Pharmacol. Exp. Ther. 270, 414-423.

Shoaib, M., Stolerman, I.P., 1999. Plasma nicotine and cotinine levels following intravenous nicotine self-administration in rats. Psychopharmacology (Berl.) 143, 318-321.

Siemer, C., Smiljakovic, T., Bhojwani, M., Leiding, C., Kanitz, W., Kubelka, M., Tomek, W., 2009. Analysis of mRNA associated factors during bovine oocyte maturation and early embryonic development. Mol. Reprod. Dev. 76, 1208-1219.

Silva, J.R.V., van den Hurk, R., van Tol, H.T.A., Roelen, B.A.J., Figueiredo, J.R., 2005. Expression of growth differentiation factor 9 (GDF9), bone morphogenetic protein 15 (BMP15), and BMP receptors in the ovaries of goats. Mol. Reprod. Dev. 70, 11-19.

Simon, A.M., Goodenough, D.A., Li, E., Paul, D.L., 1997. Female infertility in mice lacking connexin 37. Nature 385, 525-529.

Smith, C.G., Besch, N.F., Smith, R.G., Besch, P.K., 1979. Effect of tetrahydrocannabinol on the hypothalamic-pituitary axis in the ovariectomized rhesus monkey. Fertil. Steril. 31, 335-339.

Solan, J.L., Lampe, P.D., 2007. Key Connexin 43 Phosphorylation Events Regulate the Gap Junction Life Cycle. J. Membr. Biol. 217, 35-41.

Solan, J.L., Lampe, P.D., 2009. Connexin 43 phosphorylation: structural changes and biological effects. Biochem. J. 419, 261-272.

Solan, J.L., Marquez-Rosado, L., Sorgen, P.L., Thornton, P.J., Gafken, P.R., Lampe, P.D., 2007. Phosphorylation at S365 is a gatekeeper event that changes the structure of Cx43 and prevents down-regulation by PKC. J. Cell Biol. 179, 1301-1309.

Solovyeva, E.V., Hayashi, M., Margi, K., Barkats, C., Klein, C., Amsterdam, A., Hsueh, A.J., Tsafriri, A., 2000. Growth differentiation factor-9 stimulates rat theca-interstitial cell androgen biosynthesis. Biol. Reprod. 63, 1214-1218.

Steeves, T., Gardner, D., 1999. Metabolism of Glucose, Pyruvate, and Glutamine During the Maturation of Oocytes Derived From Pre-Pubertal and Adult Cows. Mol. Reprod. Dev. 54, 92-101.

Stojkovic, M., Machado, S.A., Stojkovic, P., Zakhartchenko, V., Hutzler, P., Gonçalves, P.B., Wolf, E., 2001. Mitochondrial distribution and adenosine triphosphate content of bovine oocytes before and after in vitro maturation: correlation with morphological 
criteria and developmental capacity after in vitro fertilization and culture. Biol. Reprod. 64, 904-909.

Su, Y., Sugiura, K., Eppig, J., 2009. Mouse oocyte control of granulosa cell development and function: paracrine regulation of cumulus cell metabolism. Semin. Reprod. Med. 27, $32-42$.

Su, Y.-Q., Sugiura, K., Wigglesworth, K., O’Brien, M.J., Affourtit, J.P., Pangas, S.A., Matzuk, M.M., Eppig, J.J., 2008. Oocyte regulation of metabolic cooperativity between mouse cumulus cells and oocytes: BMP15 and GDF9 control cholesterol biosynthesis in cumulus cells. Development 135, 111-121.

Su, Y.-Q., Wigglesworth, K., Pendola, F.L., O’Brien, M.J., Eppig, J.J., 2002. Mitogen-activated protein kinase activity in cumulus cells is essential for gonadotropin-induced oocyte meiotic resumption and cumulus expansion in the mouse. Endocrinology 143, 22212232.

Su, Y.Q., Wu, X., O’Brien, M.J., Pendola, F.L., Denegre, J.N., Matzuk, M.M., Eppig, J.J., 2004. Synergistic roles of BMP15 and GDF9 in the development and function of the oocytecumulus cell complex in mice: genetic evidence for an oocyte-granulosa cell regulatory loop. Dev. Biol. 276, 64-73.

Sugiura, K., Pendola, F.L., Eppig, J.J., 2005. Oocyte control of metabolic cooperativity between oocytes and companion granulosa cells: energy metabolism. Dev. Biol. 279, 20-30.

Sugiura, K., Su, Y.-Q., Diaz, F.J., Pangas, S.A., Sharma, S., Wigglesworth, K., O’Brien, M.J., Matzuk, M.M., Shimasaki, S., Eppig, J.J., 2008. Oocyte-derived BMP15 and FGFs cooperate to promote glycolysis in cumulus cells. Development 135, 786-786.

Susan-Resiga, D., Essalmani, R., Hamelin, J., Asselin, M.-C., Benjannet, S., Chamberland, A., Day, R., Szumska, D., Constam, D., Bhattacharya, S., Prat, A., Seidah, N.G., 2011. Furin is the major processing enzyme of the cardiac-specific growth factor bone morphogenetic protein 10. J. Biol. Chem. 286, 22785-22794.

Sutton, M.L., Cetica, P.D., Beconi, M.T., Kind, K.L., Gilchrist, R.B., Thompson, J.G., 2003. Influence of oocyte-secreted factors and culture duration on the metabolic activity of bovine cumulus cell complexes. Reprod. Camb. Engl. 126, 27-34. 
Sutton-McDowall, M.L., 2004. Cumulus expansion and glucose utilisation by bovine cumulusoocyte complexes during in vitro maturation: the influence of glucosamine and follicle-stimulating hormone. Reproduction 128, 313-319.

Sutton-McDowall, M.L., Gilchrist, R.B., Thompson, J.G., 2005. Effect of hexoses and gonadotrophin supplementation on bovine oocyte nuclear maturation during in vitro maturation in a synthetic follicle fluid medium. Reprod. Fertil. Dev. 17, 407-415.

Sutton-McDowall, M.L., Gilchrist, R.B., Thompson, J.G., 2010. The pivotal role of glucose metabolism in determining oocyte developmental competence. Reproduction 139, 685-695.

Sutton-McDowall, M.L., Mitchell, M., Cetica, P., Dalvit, G., Pantaleon, M., Lane, M., Gilchrist, R.B., Thompson, J.G., 2006. Glucosamine supplementation during in vitro maturation inhibits subsequent embryo development: possible role of the hexosamine pathway as a regulator of developmental competence. Biol. Reprod. 74, 881-888.

Swaab, D.F., Pool, C.W., Van Leeuwen, F.W., 1977. Can specificity ever be proved in immunocytochemical staining. J. Histochem. Cytochem. Off. J. Histochem. Soc. 25, 388-391.

Tatemoto, H., Sakurai, N., Muto, N., 2000. Protection of porcine oocytes against apoptotic cell death caused by oxidative stress during In vitro maturation: role of cumulus cells. Biol. Reprod. 63, 805-810.

Teilmann, S.C., 2005. Differential expression and localisation of connexin-37 and connexin-43 in follicles of different stages in the 4-week-old mouse ovary. Mol. Cell. Endocrinol. 234, 27-35.

Teixeira Filho, F.L., Baracat, E.C., Lee, T.H., Suh, C.S., Matsui, M., Chang, R.J., Shimasaki, S., Erickson, G.F., 2002. Aberrant expression of growth differentiation factor-9 in oocytes of women with polycystic ovary syndrome. J. Clin. Endocrinol. Metab. 87, 1337-1344.

Thomas, R., Armstrong, D., Gilchrist, R., 2002. Differential Effects of Specific Phosphodiesterase Isoenzyme Inhibitors on Bovine Oocyte Meiotic Maturation. Dev. Biol. 244, 213-225.

Thomas, R.E., Armstrong, D., Gilchrist, R., 2004. Bovine Cumulus Cell-Oocyte Gap Junctional Communication During In Vitro Maturation in Response to Manipulation of CellSpecific Cyclic Adenosine 3', 5'-Monophosophate Levels. Biol. Reprod. 70, 548-556. 
Tong, D., Li, T., Naus, K., Bai, D., Kidder, G., 2007. In vivo analysis of undocked connexin43 gap junction hemichannels in ovarian granulosa cells. J. Cell Sci. 120, 4016-4024.

Topp, L., Hando, J., Dillon, P., Roche, A., Solowij, N., 1999. Ecstasy use in Australia: patterns of use and associated harm. Drug Alcohol Depend. 55, 105-115.

Tornell, J., Billig, H., Hillensjo, T., 1990. Resumption of rat oocyte meiosis is paralleled by a decrease in guanosine $3^{\prime}, 5^{\prime}$-cyclic monophosphate (cGMP) and is inhibited by microinjection of cGMP. Acta Physiol. Scand. 139, 511-517.

Tosti, E., 2006. Calcium ion currents mediating oocyte maturation events. Reprod. Biol. Endocrinol. RBE 4, 26-35.

Tosti, E., Boni, R., Cuomo, A., 2000. Ca2+ current activity decreases during meiotic progression in bovine oocytes. Am. J. Physiol.- Cell Physiol. 279, 1795-1800.

Tsafriri, A., Chun, S., Zhang, R., Hsueh, A., Conti, M., 1996. Oocyte maturation involves compartmentalization and opposing changes of cAMP levels in follicular somatic and germ cells: studies using selective phosphodiesterase inhibitors. Dev. Biol. 178, 393402.

Tsai, C.-H., Yeh, H.-I., Tian, T.-Y., Lee, Y.-N., Lu, C.-S., Ko, Y.-S., 2004. Down-regulating effect of nicotine on connexin43 gap junctions in human umbilical vein endothelial cells is attenuated by statins. Eur. J. Cell Biol. 82, 589-595.

Tsukaguchi, H., Shayakul, C., Berger, U.V., Mackenzie, B., Devidas, S., Guggino, W.B., van Hoek, A.N., Hediger, M.A., 1998. Molecular characterization of a broad selectivity neutral solute channel. J. Biol. Chem. 273, 24737-24743.

U.S. Department of Health and Human Services, 2006. National Institute on Drug Abuse Research Report Series: MDMA (Ecstasy) Abuse [WWW Document]. Natl. Inst. Drug Abuse. URL http://www.drugabuse.gov/publications/research-reports/mdmaecstasy-abuse (accessed 5.29.13).

U.S. Department of Health and Human Services, 2010. National Institute on Drug Abuse Research Report Series: Marijuana Abuse [WWW Document]. Natl. Inst. Drug Abuse. URL http://www.drugabuse.gov/publications/research-reports/marijuana-abuse (accessed 5.29.12).

Upham, B.L., Rummel, A.M., Carbone, J.M., Trosko, J.E., Ouyang, Y., Crawford, R.B., Kaminski, N.E., 2003. Cannabinoids inhibit gap junctional intercellular communication and activate ERK in a rat liver epithelial cell line. Int. J. Cancer J. Int. Cancer 104, 12-18. 
Upton, G., 1992. Fisher's Exact Test. J. R. Stat. Soc., Statistics in Society 155, 395-402.

Van den Hurk, R., Abir, R., Telfer, E., Bevers, M., 2000. Primate and bovine immature oocytes and follicles as sources of fertilizable oocytes. Hum. Reprod. Update 6, 457-474.

Van Thiel, D.H., Gavaler, J.S., Lester, R., Sherins, R.J., 1978. Alcohol-induced ovarian failure in the rat. J. Clin. Invest. 61, 624-632.

Vanderhyden, B., Caron, P., Buccione, R., Eppig, J., 1990. Developmental pattern of the secretion of cumulus expansion-enabling factor by mouse oocytes and the role of oocytes in promoting granulosa cell differentiation. Dev. Biol. 140, 307-317.

Vanderhyden, B., Macdonald, E., 1998. Mouse oocytes regulate granulosa cell steroidogenesis throughout follicular development. Biol. Reprod. 59, 1296-1301.

Vanderhyden, B.C., 1992. Mouse oocytes promote proliferation of granulosa cells from preantral and antral follicles in vitro. Biol. Reprod. 46, 1196-1204.

Vitt, U.A., Hayashi, M., Klein, C., Hsueh, A.J., 2000. Growth differentiation factor-9 stimulates proliferation but suppresses the follicle-stimulating hormone-induced differentiation of cultured granulosa cells from small antral and preovulatory rat follicles. Biol. Reprod. 62, 370-377.

Voet, D., Voet, J., 2004. Biochemistry, 3rd ed. John Wiley \& Sons, Hoboken, NJ.

Vom Saal, F.S., Akingbemi, B.T., Belcher, S.M., Birnbaum, L.S., Crain, D.A., Eriksen, M., Farabollini, F., Guillette, L.J., Jr, Hauser, R., Heindel, J.J., Ho, S.-M., Hunt, P.A., Iguchi, T., Jobling, S., Kanno, J., Keri, R.A., Knudsen, K.E., Laufer, H., LeBlanc, G.A., Marcus, M., McLachlan, J.A., Myers, J.P., Nadal, A., Newbold, R.R., Olea, N., Prins, G.S., Richter, C.A., Rubin, B.S., Sonnenschein, C., Soto, A.M., Talsness, C.E., Vandenbergh, J.G., Vandenberg, L.N., Walser-Kuntz, D.R., Watson, C.S., Welshons, W.V., Wetherill, Y., Zoeller, R.T., 2007. Chapel Hill bisphenol A expert panel consensus statement: integration of mechanisms, effects in animals and potential to impact human health at current levels of exposure. Reprod. Toxicol. Elmsford N 24, 131-138.

Walton, K.L., Makanji, Y., Wilce, M.C., Chan, K.L., Robertson, D.M., Harrison, C.A., 2009. A common biosynthetic pathway governs the dimerization and secretion of inhibin and related transforming growth factor $\beta$ (TGF $\beta$ ) ligands. J. Biol. Chem. 284, 9311-9320.

Wang, H., Tong, D., El-Gehani, F., Tekpetey, F., Kidder, G., 2009. Connexin expression and gap junctional coupling in human cumulus cells: contribution to embryo quality. J. Cell. Mol. Med. 3, 972-984. 
Wang, Q., Chi, M., Schedl, T., Moley, K., 2012. An intercellular pathway for glucose transport into mouse oocytes. Am. J. Physiol. - Endocrinol. Metab. 302, E1511-1518.

Waylen, A., Metwally, M., Jones, G., Wilkinson, A., Ledger, W., 2009. Effects of cigarette smoking upon clinical outcomes of assisted reproduction: a meta-analysis. Hum. Reprod. Update 15, 31-44.

Webb, R., Marshall, F., Swann, K., Carroll, J., 2002. Follicle-stimulating hormone induces a gap junction-dependent dynamic change in [CAMP] and protein kinase $A$ in mammalian oocytes. Dev. Biol. 246, 441-454.

Weiss, T., Eckert, A., 1989. Cotinine levels in follicular fluid and serum of IVF patients: effect on granulosa-luteal cell function in vitro. Hum. Reprod. Oxf. Engl. 4, 482-485.

Wert, S., Larsen, W., 1989. Meiotic Resumption and Gap Junction Modulation in the Cultured Rat Cumulus-Oocyte Complex. Gamete Res. 22, 143-162.

Wiesen, J.F., Midgley, A.R., Jr, 1994. Expression of connexin 43 gap junction messenger ribonucleic acid and protein during follicular atresia. Biol. Reprod. 50, 336-348.

Williams, S.A., Blache, D., Martin, G.B., Foot, R., Blackberry, M.A., Scaramuzzi, R.J., 2001. Effect of nutritional supplementation on quantities of glucose transporters 1 and 4 in sheep granulosa and theca cells. Reprod. Camb. Engl. 122, 947-956.

Willingham, M.C., 1999. Conditional epitopes. is your antibody always specific? J. Histochem. Cytochem. Off. J. Histochem. Soc. 47, 1233-1236.

Xu, J., Osuga, Y., Yano, T., Morita, Y., Tang, X., Fujiwara, T., Takai, Y., Matsumi, H., Koga, K., Taketani, Y., Tsutsumi, O., 2002. Bisphenol A induces apoptosis and G2-to-M arrest of ovarian granulosa cells. Biochem. Biophys. Res. Commun. 292, 456-462.

Yadav, M., 2010. The role of steroids, growth factors and cAMP stimulators on the gap junction activity in cumulus oocyte complexes in the rat. VUW Masters Thesis.

Yan, C., Wang, P., DeMayo, J., DeMayo, F.J., Elvin, J.A., Carino, C., Prasad, S.V., Skinner, S.S., Dunbar, B.S., Dube, J.L., Celeste, A.J., Matzuk, M.M., 2001. Synergistic roles of bone morphogenetic protein 15 and growth differentiation factor 9 in ovarian function. Mol. Endocrinol. Baltim. Md 15, 854-866.

Ye, X.F., Chen, S.B., Wang, L.Q., Zhao, Y.C., Lv, X.F., Liu, M.J., Huang, J.C., 2010. Caffeine and dithiothreitol delay ovine oocyte ageing. Reprod. Fertil. Dev. 22, 1254-1261. 
Yogo, K., Ogawa, T., Akiyama, M., Ishida, N., Takeya, T., 2002. Identification and functional analysis of novel phosphorylation sites in $\mathrm{Cx} 43$ in rat primary granulosa cells. FEBS Lett. 531, 132-136.

Yoshino, O., McMahon, H.E., Sharma, S., Shimasaki, S., 2006. A unique preovulatory expression pattern plays a key role in the physiological functions of BMP-15 in the mouse. Proc. Natl. Acad. Sci. 103, 10678-10683.

Zeilmaker, G., Verhamme, C., 1974. Observations on rat oocyte maturation in vitro: morphology and energy requirements. Biol. Reprod. 11, 145-152.

Zenzes, M.T., Reed, T.E., 1998. Interovarian differences in levels of cotinine, a major metabolite of nicotine, in women undergoing IVF who are exposed to cigarette smoke. J. Assist. Reprod. Genet. 15, 99-103.

Zenzes, M.T., Reed, T.E., Wang, P., Klein, J., 1996. Cotinine, a major metabolite of nicotine, is detectable in follicular fluids of passive smokers in in vitro fertilization therapy. Fertil. Steril. 66, 614-619.

Zhang, M., Su, Y., Sugiura, K., Wigglesworth, K., Xia, G., Eppig, J., 2011. Estradiol Promotes and Maintains Cumulus Cell Expression of Natriuretic Peptide Receptor 2 (NPR2) and Meiotic Arrest in Mouse Oocytes In Vitro. Endocrinology 152, 4377-4385.

Zhang, M., Su, Y.-Q., Sugiura, K., Xia, G., Eppig, J.J., 2010. Granulosa cell ligand NPPC and its receptor NPR2 maintain meiotic arrest in mouse oocytes. Science 330, 366-369.

Zhou, J., Bievre, M., Bondy, C.A., 2000. Reduced GLUT1 expression in Igf1-/- null oocytes and follicles. Growth Horm. IGF Res. Off. J. Growth Horm. Res. Soc. Int. IGF Res. Soc. 10, 111-117.

Zhuo, L., Liu, Q., Liu, L., Sun, T.-Y., Wang, R.-S., Qu, G.-Q., Liu, Q., Liu, Y., Ren, L., 2013. Roles of 3,4-methylenedioxymethamphetamine (MDMA)-induced alteration of connexin43 and intracellular $\mathrm{Ca}(2+)$ oscillation in its cardiotoxicity. Toxicology $310,61-72$. 